\title{
Unmasking the Ligand Effect in Manganese-Catalyzed Hydrogenation: Mechanistic Insight and Catalytic
}

\section{Application}

\author{
Yujie Wang, ${ }^{\text {a, }}{ }^{\S}$ Lei Zhu, ${ }^{\text {b, }}{ }^{\S}$ Zhihui Shao, ${ }^{a}$ Gang Li, ${ }^{c}$ Yu Lan ${ }^{*, b}$ and Qiang Liu,a \\ ${ }^{a}$ Center of Basic Molecular Science (CBMS), Department of Chemistry, Tsinghua University, \\ Beijing 100084, China \\ bSchool of Chemistry and Chemical Engineering, Chongqing Key Laboratory of Theoretical and \\ Computational Chemistry, Chongqing University, Chongqing 400030, China \\ 'Department of Chemistry and Biochemistry, Utah State University, ozoo Old Main Hill, Logan, \\ Utah 84322-0300, United States
}




\section{Table of Contents}

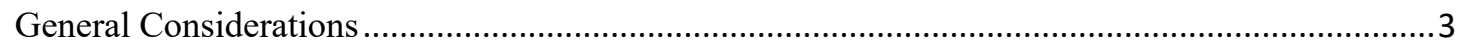

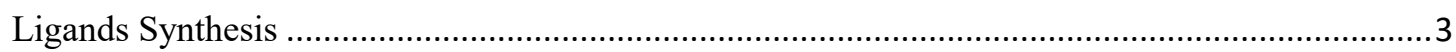

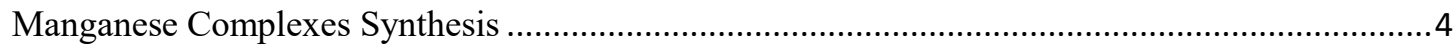

General Procedure for the Mn-Catalyzed Hydrogenation of Benzanilide 1a .................................

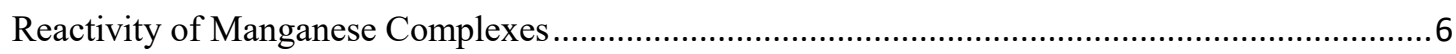

Kinetic Studies: Reaction of 1,2-Dihydroquinoline 7a with Amido Mn Complexes [Mn]-1a _.......10

Procedures for Mn-Catalyzed Hydrogenation of Methyl 4-Acetylbenzoate 2a.............................13

General Procedure for the Mn-Catalyzed Hydrogenation of $\mathrm{N}$-Heterocycles...................................14

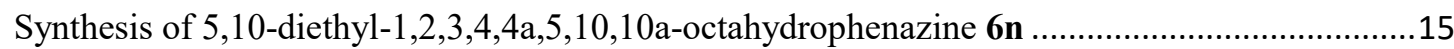

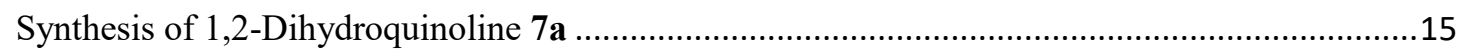

Procedures for [Mn]-1a Catalyzed Hydrogenation of Quinoline 3a ...........................................16

Procedures for Mn-Catalyzed Hydrogenation of 1,2-Dihydroquinoline 7a.................................17

Reaction of $t \mathrm{BuOK}$ with 1,2-Dihydroquinoline 7a: Experimental Evidence of Isomerization of 7a

$\ldots$

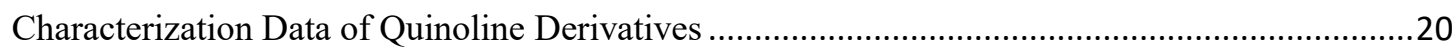

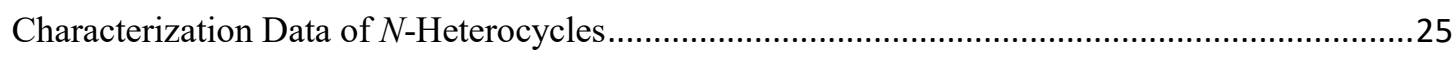

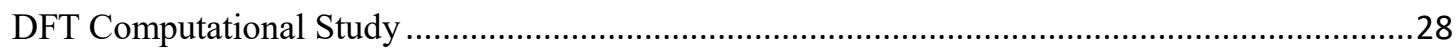

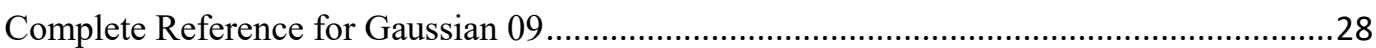

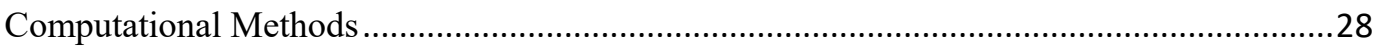

Intrinsic Reaction Coordinate (IRC) Calculation of Concerted Hydride/Proton Transfer

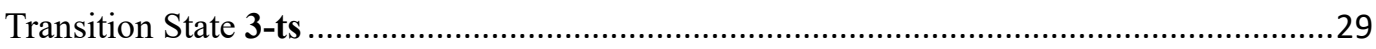

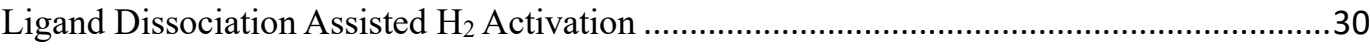

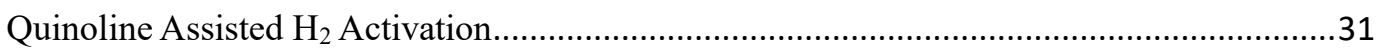

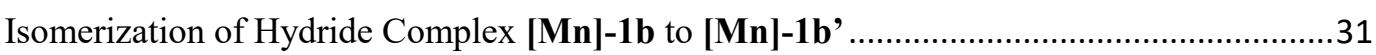

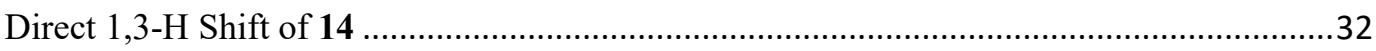

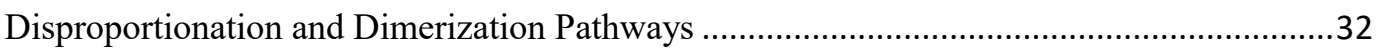

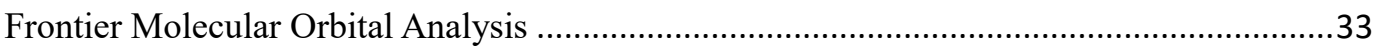

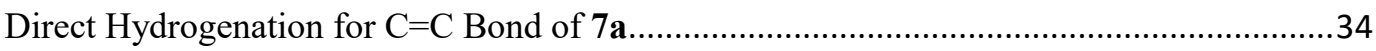

1,4-Hydrogenation of Quinoline 3a by PNP Mn-H Complex [Mn]-5b................................34

Optimized Transition State Structures (5-ts and 10-ts) ....................................................... 35

Intrinsic Reaction Coordinate (IRC) Calculation of Concerted Hydride/Proton Transfer

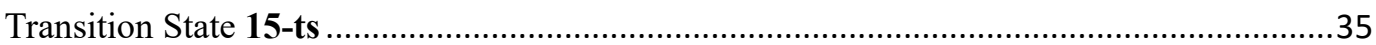

Absolute Calculation Energies, Enthalpies, and Free Energies.............................................36

B3LYP Geometries for All the Optimized Compounds and Transition State .........................38

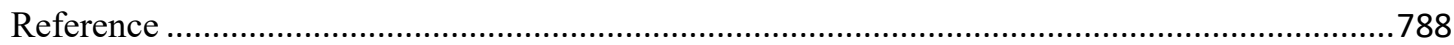

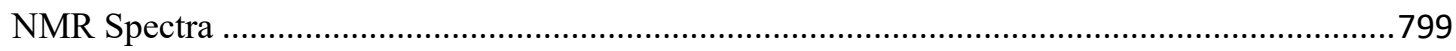




\section{General Considerations}

Air and moisture sensitive reactions were carried out in glovebox or in over-dried glassware sealed with rubber septa using standard schlenk techniques. Solvents used in moisture and oxygen sensitive reactions were firstly degassed by three freeze-pump-thaw cycles and then distilled under Ar protection after dehydration (ether solvents were dried over sodium metal). Deuterated solvents were ordered from Cambridge Isotope Laboratories, vented and distilled over calcium hydride. Most chemicals were purchased from commercial sources with purity over $95 \%$ and used without further purification. Substrates of 6-methylsulfanylquinoline $(\mathbf{3 g})^{1}, 6$-(2-phenylethynyl)quinoline $(\mathbf{3 m})^{2}, 6$-styrylquinoline $(1 \mathrm{n})^{2}$ and 2-styrylquinoline $(\mathbf{3 t})^{3}$ were synthesised following the reported protocols. Most reactons and manipulations were performed using standard Schlenk techniques. NMR spectra were received using a Bruker $400 \mathrm{MHz}$ spectrometer. Chemical shifts are reported in ppm relative to the deuterated solvent. GC analysis were carried out on an SHIMADAZU GC 2010 PLUS system. GC/MS analyses were carried out on a Thermo Trace ISQ system equipped with a Thermo SCIENTIFIC TG-5MS (30 m x $250 \mu \mathrm{m}$ x $25 \mu \mathrm{m}$ ). High resolution exact mass measurements (HRMS) were performed on Thermo SCIENTIFIC Q EXACTIVE. X-Ray crystal structure analyses were performed with a MM007HF Saturn724+system. Elemental analysis was performed by PERKIN ELMER CE-440. IR measurements were performed with a BRUKER VERTEX70 system.

\section{Ligands Synthesis}

\section{Synthesis of 2-(diphenylphosphaneyl)ethan-1-amine}

$$
\mathrm{HPPh}_{2}+n \mathrm{BuLi}+\mathrm{TMS}^{-\mathrm{N}} \mathrm{Cl}_{\mathrm{Cl}}^{\stackrel{\mathrm{TMS}}{\mathrm{THF}} \underset{\text { then } \mathrm{H}^{+} \text {and } \mathrm{OH}^{-}}{\longrightarrow} \mathrm{Ph}_{2} \mathrm{P}} \overbrace{\mathrm{NH}_{2}}
$$

In a $100 \mathrm{~mL}$ Schlenk flask, a solution of $\mathrm{HPPh}_{2}(1.86 \mathrm{~g}, 10 \mathrm{mmol})$ in $20 \mathrm{~mL}$ of anhydrous THF was cooled to $0{ }^{\circ} \mathrm{C}$, and treated dropwise with $n$ BuLi solution in hexanes $(2.5 \mathrm{M}, 4.4 \mathrm{~mL}, 11 \mathrm{mmol})$. The reaction mixture was turned into deep red color immediately and further stirred at room temperature for $2 \mathrm{~h}$. And then the solution was again cooled to $0{ }^{\circ} \mathrm{C}$ and $N, N$-bis(trimethylsilyl)-2-chloroethanamine (2. $45 \mathrm{~g}, 11 \mathrm{mmol}$ ) was added dropwise. The mixture was warmed to room temperature and then refluxed at $75{ }^{\circ} \mathrm{C}$ for 12 hours. Afterwards the reaction mixture was cooled to $0{ }^{\circ} \mathrm{C}$. Water $(5 \mathrm{~mL})$ was added, followed by $2.0 \mathrm{M} \mathrm{H}_{2} \mathrm{SO}_{4}$ solution $(6 \mathrm{~mL})$. After stirring for 1 hour at $0{ }^{\circ} \mathrm{C}$, a solution of $4.0 \mathrm{M} \mathrm{NaOH}$ solution $(7 \mathrm{~mL})$ was then added, and the mixture was stirred for another $0.5 \mathrm{~h}$. The organic layer was separated and the aqueous phase was extracted with $\mathrm{Et}_{2} \mathrm{O}(3 \times 20 \mathrm{~mL})$. Combined organic layer was dried $\left(\mathrm{Na}_{2} \mathrm{SO}_{4}\right)$, filtered and evaporated in vacuo to give 2-(diphenylphosphaneyl)ethan-1-amine as a pale yellow oil crude product without further purification $(2.06 \mathrm{~g}, 90 \%)$

Synthesis of 2-(diphenylphosphaneyl)- $N$-((1-methyl-1H-imidazol-2-yl)methyl)ethan-1-amine (L-2)

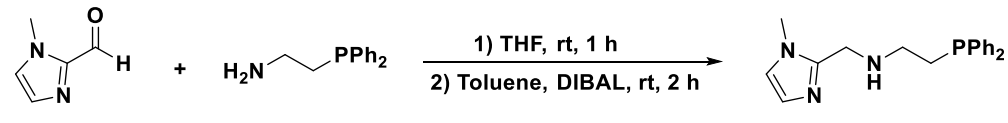


2-(diphenylphosphaneyl)ethan-1-amine $(0.29 \mathrm{~g}, 1.0 \mathrm{mmol})$ in $6 \mathrm{~mL}$ of THF was added to 1-methyl-1 $\mathrm{H}$ imidazole-2-carbaldehyde $(0.11 \mathrm{~g}, 1.0 \mathrm{mmol})$ in $6 \mathrm{~mL}$ of THF. The mixture was stirred for $1 \mathrm{~h}$ at room temperature, then evaporated. The above oil residue was dissolved in $12 \mathrm{~mL}$ of toluene and was slowly added $1.5 \mathrm{M}$ solution of DIBAL $(1.5 \mathrm{M}, 1.2 \mathrm{~mL}, 1.8 \mathrm{mmol})$ in toluene. The product solution was stirred for $2 \mathrm{~h}$ at $0{ }^{\circ} \mathrm{C}$, and then quenched with $10 \mathrm{~mL}$ of water and extracted with $\mathrm{Et}_{2} \mathrm{O}(3 \times 15 \mathrm{~mL})$. The combined organic layer was dried over $\mathrm{Na}_{2} \mathrm{SO}_{4}$, filtered and concentrated under vacuum. And the residue was purified by chromatography on silica gel to give pure desirable product as a pale yellow oil $(0.21 \mathrm{~g}$, $65 \%)$.

${ }^{1} \mathrm{H} \mathrm{NMR}\left(400 \mathrm{MHz}, \mathrm{CDCl}_{3}\right) \delta 7.41(\mathrm{ddt}, J=7.4,5.4,2.7 \mathrm{~Hz}, 4 \mathrm{H}), 7.35-7.29(\mathrm{~m}, 6 \mathrm{H}), 6.91(\mathrm{~d}, J=1.3$ $\mathrm{Hz}, 1 \mathrm{H}), 6.80(\mathrm{~d}, J=1.3 \mathrm{~Hz}, 1 \mathrm{H}), 3.82(\mathrm{~s}, 2 \mathrm{H}), 3.64$ (s, $3 \mathrm{H}), 2.86-2.74(\mathrm{~m}, 2 \mathrm{H}), 2.31-2.23$ (m, 2 $\mathrm{H}), 1.77$ (b, $1 \mathrm{H}) .{ }^{13} \mathrm{C} \mathrm{NMR}\left(101 \mathrm{MHz}, \mathrm{CDCl}_{3}\right) \delta 146.3,138.3$ (d, $\left.J=12.4 \mathrm{~Hz}\right), 132.7$ (d, $\left.J=18.7 \mathrm{~Hz}\right)$, 128.6, $128.4(\mathrm{~d}, J=6.7 \mathrm{~Hz}), 127.1,121.2,46.2(\mathrm{~d}, J=20.3 \mathrm{~Hz}), 45.6,32.7,28.9(\mathrm{~d}, J=12.4 \mathrm{~Hz}) .{ }^{31} \mathrm{P}$ $\operatorname{NMR}\left(162 \mathrm{MHz}, \mathrm{CDCl}_{3}\right) \delta-20.76$ (s).

HRMS (ESI) calcd. for $\mathrm{C}_{19} \mathrm{H}_{22} \mathrm{~N}_{3} \mathrm{P}$ [M+H] $]^{+}$: 324.1624; found: 324.1610 .

Synthesis of bis(2-(diphenylphosphaneyl)ethyl)amine (L-6)

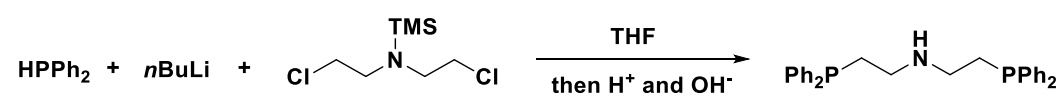

In a $100 \mathrm{~mL}$ Schlenk flask, a solution of $\mathrm{HPPh}_{2}(1.86 \mathrm{~g}, 10 \mathrm{mmol})$ in $20 \mathrm{~mL}$ of anhydrous THF was cooled to $0{ }^{\circ} \mathrm{C}$, and treated dropwise with $n$ BuLi solution in hexanes $(2.5 \mathrm{M}, 4.4 \mathrm{~mL}, 11 \mathrm{mmol})$. The reaction mixture was turned into deep red color immediately and further stirred at room temperature for $2 \mathrm{~h}$. And then the solution was again cooled to $0{ }^{\circ} \mathrm{C}$ and $N, N$-bis(2-chloroethyl)-1,1,1trimethylsilanamine $(0.96 \mathrm{~g}, 4.5 \mathrm{mmol})$ was added dropwise. The mixture was warmed to room temperature and then refluxed at $75^{\circ} \mathrm{C}$ for 12 hours. Afterwards the reaction mixture was cooled to $0{ }^{\circ} \mathrm{C}$. Water $(8 \mathrm{~mL})$ was added, followed by $2.0 \mathrm{M} \mathrm{H}_{2} \mathrm{SO}_{4}$ solution $(2.5 \mathrm{~mL})$. After stirring for 1 hour at $0{ }^{\circ} \mathrm{C}$, a solution of $4.0 \mathrm{M} \mathrm{NaOH}$ solution $(2.8 \mathrm{~mL})$ was then added, and the mixture was stirred for another 0.5 h. The organic layer was separated and the aqueous phase was extracted with $\mathrm{Et}_{2} \mathrm{O}(3 \times 20 \mathrm{~mL})$. Combined organic layer was dried $\left(\mathrm{Na}_{2} \mathrm{SO}_{4}\right)$, filtered and evaporated in vacuo, the residue was purified by chromatography on silica gel to give bis(2-(diphenylphosphaneyl)ethyl)amine product as a white solid $(1.10 \mathrm{~g}, 56 \%)$.

${ }^{1} \mathrm{H}$ NMR $\left(400 \mathrm{MHz}, \mathrm{CDCl}_{3}\right) \delta 7.39(\mathrm{td}, \mathrm{J}=7.2,3.1 \mathrm{~Hz}, 8 \mathrm{H}), 7.34-7.28(\mathrm{~m}, 12 \mathrm{H}), 2.84(\mathrm{dt}, \mathrm{J}=10.7$, $6.4 \mathrm{~Hz}, 4 \mathrm{H}), 2.49-2.32(\mathrm{~m}, 4 \mathrm{H}) .{ }^{13} \mathrm{C} \mathrm{NMR}\left(101 \mathrm{MHz}, \mathrm{CDCl}_{3}\right) \delta 136.1$ (d, $\left.J=12.2 \mathrm{~Hz}\right), 132.5$ (d, $J=$ $19.1 \mathrm{~Hz}), 129.0,128.7(\mathrm{~d}, J=6.7 \mathrm{~Hz}), 44.2(\mathrm{~d}, J=26.4 \mathrm{~Hz}), 23.8(\mathrm{~d}, J=16.1 \mathrm{~Hz}) .{ }^{31} \mathrm{P}$ NMR $(162 \mathrm{MHz}$, $\left.\mathrm{CDCl}_{3}\right) \delta-20.87(\mathrm{~s})$.

HRMS (ESI) calcd. for $\mathrm{C}_{28} \mathrm{H}_{29} \mathrm{NP}_{2}$ [M+H] $]^{+}$: 442.1848; found: 442.1842.

\section{Manganese Complexes Synthesis}

The synthesis of complexes [Mn]-1, [Mn]-3, [Mn]-5, and [Mn]-7 were reported in our previous works. ${ }^{4}$ Complexes $[\mathbf{M n}]-\mathbf{4}$, and $[\mathbf{M n}]-\mathbf{8}$ were prepared according to a reported procedure. ${ }^{5}$ 
Synthesis of $\left\{\mathrm{Mn}(\mathrm{CO})_{3}(2-(\right.$ diphenylphosphaneyl)- $N$-[(1-methyl-1H-imidazol-2-yl)methyl]ethan-1amine\} Br [Mn]-2

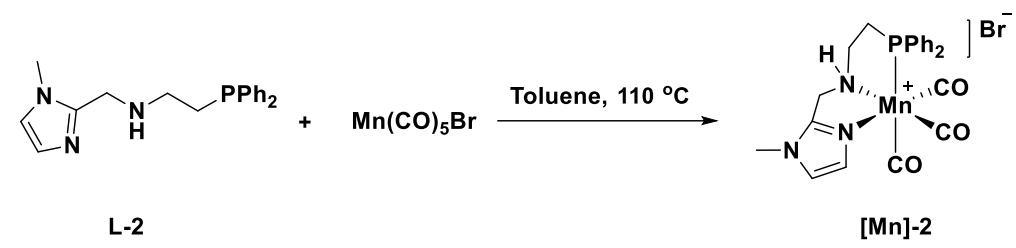

In a $50 \mathrm{~mL}$ Schlenk flask, a solution of $\left[\mathrm{MnBr}(\mathrm{CO})_{5}\right](274 \mathrm{mg}, 1 \mathrm{mmol})$ in $10 \mathrm{~mL}$ of toluene was added to a solution of 2-(diphenylphosphaneyl)- $N$-[(1-methyl-1H-imidazol-2-yl)methyl]ethan-1-amine L-2 (340 mg, $1.05 \mathrm{mmol}$ ) in $5 \mathrm{~mL}$ of toluene at room temperature. The resulting solution was then heated to $110^{\circ} \mathrm{C}$ for 12 hours. After that, yellow precipitate was formed, collected by filtration, and washed with toluene $(1 \mathrm{~mL})$ for three times. Then the filter cake was dried in vacuum to give faint yellow powder product (433 $\mathrm{mg}, 80 \%$ ).

${ }^{1} \mathrm{H}$ NMR (400 MHz, DMSO-d $) \delta 7.76(\mathrm{t}, J=7.6 \mathrm{~Hz}, 2 \mathrm{H}), 7.55(\mathrm{~m}, 3 \mathrm{H}), 7.45-7.34(\mathrm{~m}, 5 \mathrm{H}), 7.07$ (s, $1 \mathrm{H}), 6.42(\mathrm{~s}, 1 \mathrm{H}), 4.32(\mathrm{~d}, J=16.8 \mathrm{~Hz}, 1 \mathrm{H}), 4.08(\mathrm{~d}, J=16.8 \mathrm{~Hz}, 1 \mathrm{H}), 3.56(\mathrm{~s}, 3 \mathrm{H}), 3.14(\mathrm{~m}, 1 \mathrm{H})$, $2.87-2.69$ (m, $1 \mathrm{H}), 2.27$ (m, $2 \mathrm{H}) .{ }^{13} \mathrm{C}$ NMR (101 MHz, DMSO- $\left.d_{6}\right) \delta 149.8,132.7,132.3,132.0,131.9$, 131.7, 131.4, 131.0, 130.9, 129.7 (d, $J=9.5 \mathrm{~Hz}), 129.4$ (d, $J=9.3 \mathrm{~Hz}), 128.1,125.2,54.9(\mathrm{~d}, J=11.4$ $\mathrm{Hz}), 49.7,34.7,22.5$ (d, $J=22.6 \mathrm{~Hz}) .{ }^{31} \mathrm{P}$ NMR (162 MHz, DMSO- $\left.d_{6}\right) \delta 63.97$ (s).

HRMS (ESI) calcd. for $\mathrm{C}_{22} \mathrm{H}_{22} \mathrm{BrMnN}_{3} \mathrm{O}_{3} \mathrm{P}$ [M-Br] ${ }^{+}$: 462.0774 ; found: 462.0775 .

Synthesis of Bromodicarbonyl(bis(2-(diphenylphosphaneyl)ethyl)amine)manganese [Mn]-6

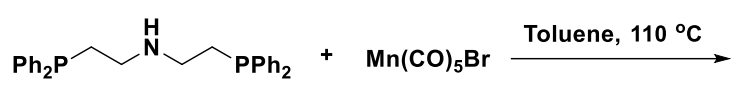

L-6

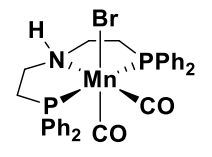

[Mn]-6

In a $50 \mathrm{~mL}$ Schlenk flask, a solution of $\left[\mathrm{MnBr}(\mathrm{CO})_{5}\right](274 \mathrm{mg}, 1 \mathrm{mmol})$ in $10 \mathrm{~mL}$ of toluene was added to a solution of bis(2-(diphenylphosphaneyl)ethyl)amine L-6 (463 mg, $1.05 \mathrm{mmol})$ in $5 \mathrm{~mL}$ of toluene at room temperature. The resulting solution was then heated to $110^{\circ} \mathrm{C}$ for 20 hours. After that, it was cooled to room temperature and concentrated in vacuo $(433 \mathrm{mg}, 80 \%)$. Hexane $(20 \mathrm{~mL})$ was added to the reaction Schlenk flask and then the suspension was filtered. The crude precipitate was extracted with dichloromethane and the resulted filtrate solution was concentrated under reduced pressure and dried to afford the complex [Mn]-6 as a yellow powder (391 mg, 62\%). ${ }^{6}$

${ }^{1} \mathrm{H}$ NMR (400 MHz, $\left.\mathrm{CD}_{2} \mathrm{Cl}_{2}\right) \delta 7.90(\mathrm{~m}, J=5.9 \mathrm{~Hz}, 4 \mathrm{H}), 7.59(\mathrm{~m}, 4 \mathrm{H}), 7.52-7.22(\mathrm{~m}, 12 \mathrm{H}), 3.67$ (m, $2 \mathrm{H}), 3.54$ (br, $1 \mathrm{H}), 3.27$ (m, $2 \mathrm{H}), 2.77(\mathrm{~m}, 2 \mathrm{H}), 2.41(\mathrm{~m}, 2 \mathrm{H}) .{ }^{13} \mathrm{C} \mathrm{NMR}\left(101 \mathrm{MHz}, \mathrm{CD}_{2} \mathrm{Cl}_{2}\right) \delta 138.0$ $(\mathrm{t}, J=18.9 \mathrm{~Hz}), 135.5(\mathrm{t}, J=18.8 \mathrm{~Hz}), 133.9(\mathrm{t}, J=4.8 \mathrm{~Hz}), 130.7(\mathrm{t}, J=5.0 \mathrm{~Hz}), 130.3,129.7,129.1(\mathrm{t}$, $J=4.2 \mathrm{~Hz}), 128.8(\mathrm{t}, J=4.5 \mathrm{~Hz}), 53.1(\mathrm{t}, J=4.8 \mathrm{~Hz}), 28.5(\mathrm{t}, J=8.8 \mathrm{~Hz}) .{ }^{31} \mathrm{P} \mathrm{NMR}\left(162 \mathrm{MHz}, \mathrm{CD}_{2} \mathrm{Cl}_{2}\right)$ $\delta 69.65(\mathrm{~s})$.

\section{General Procedure for the Mn-Catalyzed Hydrogenation of Benzanilide 1a}

Under the argon atmosphere glovebox, benzanilide 1a (49.3 mg, $0.25 \mathrm{mmol}), t \mathrm{BuOK}(2.8 \mathrm{mg}, 0.025$ mmol, $10 \mathrm{~mol} \%)$, Mn catalysts $(0.005 \mathrm{mmol}, 2 \mathrm{~mol} \%)$, cyclohexane (2 $\mathrm{mL})$ were added sequentially to 
the vial with a magnetic stir bar, which was capped with a septum equipped with a syringe. The vial was placed in the alloy plate, which was then transfered into a predried autoclave. Once sealed, the autoclave was purged with hydrogen for three times, followed by pressurized to 30 bar of $\mathrm{H}_{2}$ and heated at $110{ }^{\circ} \mathrm{C}$ for $16 \mathrm{~h}$. After reaction, the autoclave was cooled with ice-water bath, and then depressurized. The reaction mixture was diluted by $2 \mathrm{~mL}$ of $\mathrm{CH}_{2} \mathrm{Cl}_{2}$. The conversion of benzanilide (1a) and yield of aniline (1b) and benzyl alcohol (1c) was determined by GC with dodecane as the internal standard.

Table S1. Control Experiment and Homogenity Tests for the Mn-Catalyzed Hydrogenation of Benzanilide 1a ${ }^{a}$

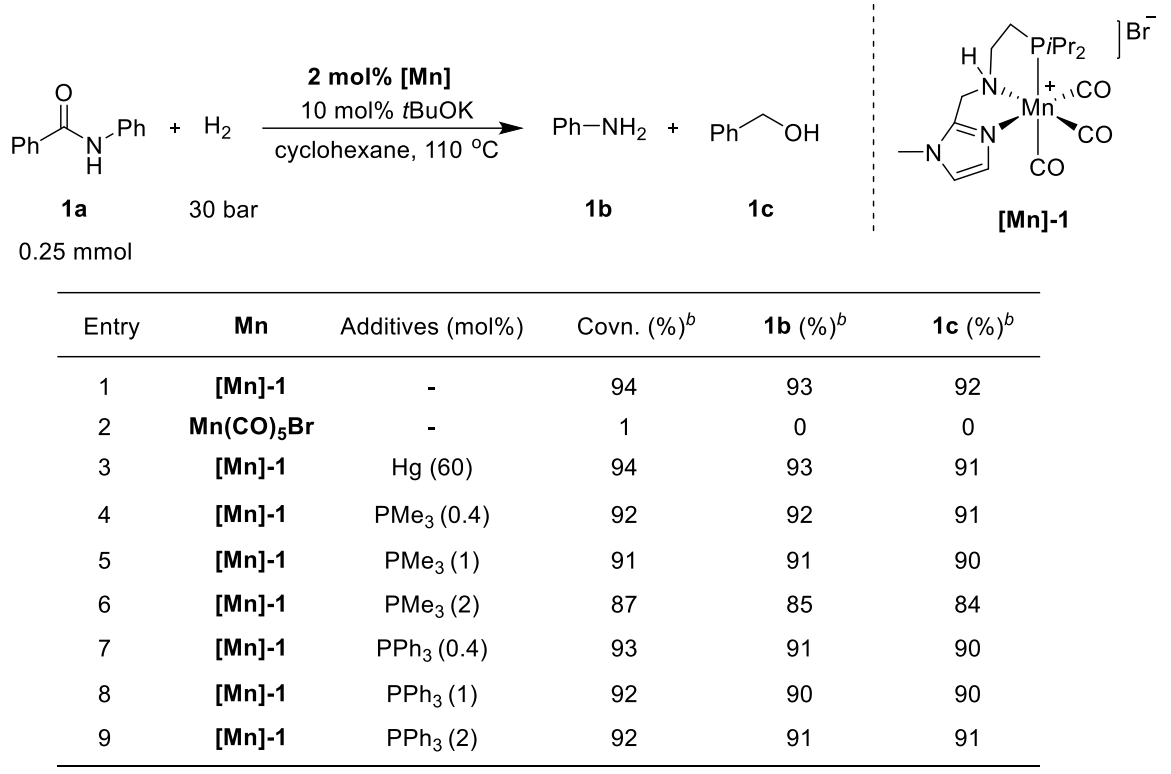

${ }^{a}$ Reaction conditions: benzanilide $1 \mathrm{a}(0.25 \mathrm{mmol})$, Mn catalysts $(2 \mathrm{~mol} \%), t \mathrm{BuOK}(10 \mathrm{~mol} \%)$, cyclohexane $(2 \mathrm{~mL})$, additives (phosphines or mercury), $110{ }^{\circ} \mathrm{C}, \mathrm{H}_{2}$ (30 bar), $16 \mathrm{~h} .{ }^{b}$ Conversion of $\mathbf{1 a}$ and yield of $\mathbf{1 b}$ and $\mathbf{1 c}$ determined by GC using dodecane as an as internal standard.

Under the argon atmosphere glovebox, benzanilide 1a (49.3 mg, $0.25 \mathrm{mmol}), t \mathrm{BuOK}(2.8 \mathrm{mg}, 0.025$ mmol, $10 \mathrm{~mol} \%)$, Mn catalysts $(0.005 \mathrm{mmol}, 2 \mathrm{~mol} \%$ ), cyclohexane $(2 \mathrm{~mL})$, and varying amounts of poisoning agents $\left(\mathrm{Hg}, \mathrm{PMe}_{3}, \mathrm{PPh}_{3}\right)$ were added sequentially to the vial with a magnetic stir bar, which was capped with a septum equipped with a syringe. The next operations were same as the general procedure above.

\section{Reactivity of Manganese Complexes}

\section{Amido Complex [Mn]-1a}

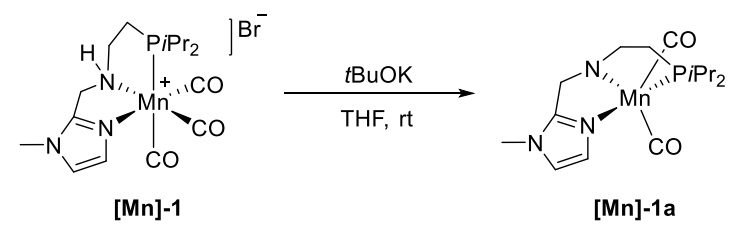

A dried $25 \mathrm{~mL}$ Schlenk flask was charged with [Mn]-1 (190 mg, $0.4 \mathrm{mmol})$, potassium tert-butoxide (50 $\mathrm{mg}, 0.45 \mathrm{mmol})$ and THF (6 mL) in sequence. The solution soon turned into dark red. The reaction was 
stirred for 1 hour. After the volatiles were removed under reduced pressure, diethyl ether $(10 \mathrm{~mL})$ was added and the resulted solution was filtered to remove unsolved salts. After that, the diethyl ether solution was concentrated to dryness followed by the addition of hexane $(10 \mathrm{~mL})$ and then the suspension was filtered. The shiny red solid precipitate was collected and dried to give [Mn]-1a (97 mg, 66\%).

${ }^{1} \mathrm{H}$ NMR (400 MHz, $\left.\mathrm{C}_{6} \mathrm{D}_{6}\right) \delta 7.33(\mathrm{~s}, 1 \mathrm{H}), 5.91(\mathrm{~s}, 1 \mathrm{H}), 3.39$ (dt, $\left.J=12.6,6.7 \mathrm{~Hz}, 2 \mathrm{H}\right), 3.14(\mathrm{~d}, J=2.9$ $\mathrm{Hz}, 2 \mathrm{H}$ ), 2.24 (s, $3 \mathrm{H}$ ), 2.13 (h, $J=7.2 \mathrm{~Hz}, 2 \mathrm{H}$ ), 1.90 (q, $J=7.4 \mathrm{~Hz}, 2 \mathrm{H}$ ), 1.28 (dd, $J=14.8,7.0 \mathrm{~Hz}, 6$ $\mathrm{H}), 1.16(\mathrm{dd}, J=12.9,6.9 \mathrm{~Hz}, 6 \mathrm{H}) .{ }^{13} \mathrm{C}$ NMR $\left(101 \mathrm{MHz}, \mathrm{C}_{6} \mathrm{D}_{6}\right) \delta 153.4,130.1,120.0,59.5(\mathrm{~d}, J=10.9$ $\mathrm{Hz}), 57.5(\mathrm{~d}, J=5.1 \mathrm{~Hz}), 32.3,26.5(\mathrm{~d}, J=21.2 \mathrm{~Hz}), 25.4(\mathrm{~d}, J=14.0 \mathrm{~Hz}), 18.1(\mathrm{~d}, J=3.6 \mathrm{~Hz}), 17.6 .{ }^{31} \mathrm{P}$ NMR (162 MHz, $\left.\mathrm{C}_{6} \mathrm{D}_{6}\right) \delta 124.58(\mathrm{~s})$.

Elemental analysis calcd (\%) for: C, 49.32; H, 6.90; N, 11.50; found: C, 49.00; H, 7.21; N, 11.05 ATR-FTIR (solid, $\left.\mathrm{cm}^{-1}\right): v(\mathrm{CO})=1801,1882$

HRMS (ESI) calcd. for $\mathrm{C}_{15} \mathrm{H}_{25} \mathrm{MnN}_{3} \mathrm{O}_{2} \mathrm{P}[\mathrm{M}+\mathrm{H}]^{+}: 366.1138$; found: 366.1142 .

Single crystal suitable for X-ray diffraction measurements were crystallized from diethyl ether solution at $-35^{\circ} \mathrm{C}$ (Figure $\mathrm{S} 1$ ).

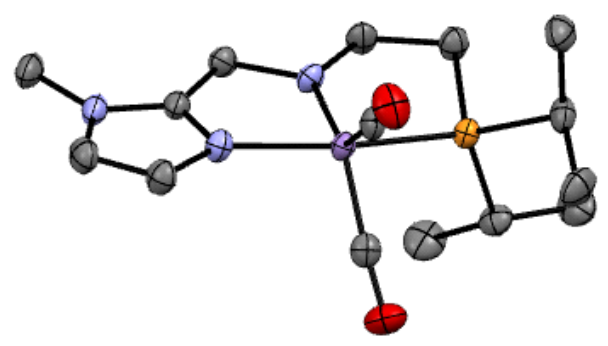

Figure S1. Crystal structure of complex [Mn]-1a

\section{Amido Complex [Mn]-2a}

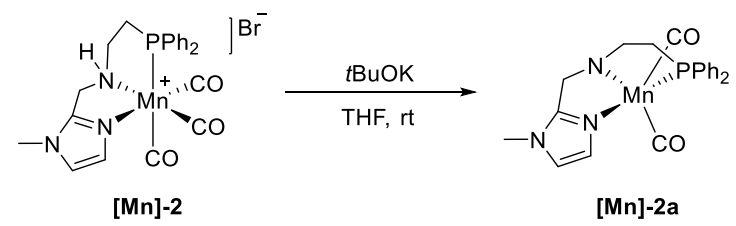

A dried $25 \mathrm{~mL}$ Schlenk flask was charged with [Mn]-2 (108 mg, $0.2 \mathrm{mmol})$, potassium tert-butoxide ( 25 $\mathrm{mg}, 0.22 \mathrm{mmol})$ and THF $(4 \mathrm{~mL})$ in sequence. The solution soon turned into dark red. The reaction was stirred for 1 hour. After the volatiles were removed under reduced pressure, benzene $(10 \mathrm{~mL})$ was added and the resulted solution was filtered to remove unsolved salts. After that, the benzene solution was concentrated to dryness followed by the addition of hexane $(10 \mathrm{~mL})$ and then the suspension was filtered. The shiny red solid precipitate was collected and dried to give [Mn]-2a (45 mg, 52\%).

${ }^{1} \mathrm{H}$ NMR $\left(400 \mathrm{MHz}, \mathrm{C}_{6} \mathrm{D}_{6}\right) \delta 7.79(\mathrm{t}, \mathrm{J}=8.8 \mathrm{~Hz}, 4 \mathrm{H}), 7.39(\mathrm{~s}, 1 \mathrm{H}), 7.15-7.00(\mathrm{~m}, 8 \mathrm{H}), 5.89(\mathrm{~s}, 1 \mathrm{H})$, 3.36 (d, J = 16.4 Hz, 2 H), 3.10 (s, $2 \mathrm{H}), 2.72-2.62$ (m, $2 \mathrm{H}), 2.21$ (s, $3 \mathrm{H}) .{ }^{13} \mathrm{C} \mathrm{NMR}\left(101 \mathrm{MHz}, \mathrm{C}_{6} \mathrm{D}_{6}\right) \delta$ 153.9, 137.9, 137.6, 132.6 (d, J = 10.5 Hz), 130.9, 129.6 (d, J = 2.1 Hz), 128.6, 128.5, 120.4, 57.9 (d, J $=5.0 \mathrm{~Hz}), 56.7(\mathrm{~d}, \mathrm{~J}=12.1 \mathrm{~Hz}), 36.0(\mathrm{~d}, \mathrm{~J}=21.3 \mathrm{~Hz}), 32.6 .{ }^{31} \mathrm{P} \mathrm{NMR}\left(162 \mathrm{MHz}, \mathrm{C}_{6} \mathrm{D}_{6}\right) \delta 105.08(\mathrm{~s})$.

ATR-FTIR (solid, $\left.\mathrm{cm}^{-1}\right): v(\mathrm{CO})=1806,1888$

HRMS (ESI) calcd. for $\mathrm{C}_{21} \mathrm{H}_{21} \mathrm{MnN}_{3} \mathrm{O}_{2} \mathrm{P}[\mathrm{M}+\mathrm{H}]^{+}$: 434.0825; found: 434.0819 . 


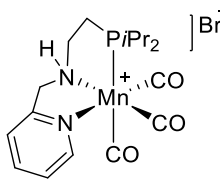

$[\mathrm{Mn}]-3$

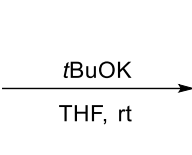
A dried $25 \mathrm{~mL}$ Schlenk flask was charged with [Mn]-3 (94 mg, $0.2 \mathrm{mmol})$, potassium tert-butoxide (25
$\mathrm{mg}, 0.22 \mathrm{mmol})$ and THF ( $4 \mathrm{~mL})$ in sequence. The solution soon turned into dark red. The reaction was stirred for 1 hour. After the volatiles were removed under reduced pressure, benzene $(10 \mathrm{~mL})$ was added and the resulted solution was filtered to remove unsolved salts. After that, the benzene solution was concentrated to dryness followed by the addition of hexane $(10 \mathrm{~mL})$ and then the suspension was filtered. The shiny red solid precipitate was collected and dried to give [Mn]-3a (36 mg, 50\%).

${ }^{1} \mathrm{H}$ NMR (400 MHz, C $\left.6 \mathrm{D}_{6}\right) \delta 9.24(\mathrm{~s}, 1 \mathrm{H}), 6.70(\mathrm{~s}, 1 \mathrm{H}), 6.46(\mathrm{~d}, \mathrm{~J}=7.6 \mathrm{~Hz}, 1 \mathrm{H}), 6.30(\mathrm{~s}, 1 \mathrm{H}), 3.83(\mathrm{~s}$, $2 \mathrm{H}), 3.48-3.08$ (m, $2 \mathrm{H}), 2.25-1.95(\mathrm{~m}, 2 \mathrm{H}), 1.82$ (q, J = 7.7 Hz, $2 \mathrm{H}), 1.16$ (ddd, J = 50.5, 14.3, 7.0 $\mathrm{Hz}, 12 \mathrm{H}) .{ }^{13} \mathrm{C}$ NMR (101 MHz, $\left.\mathrm{C}_{6} \mathrm{D}_{6}\right) \delta 166.0,154.3,133.5,120.7,118.8,69.4$ (d, J = 6.4 Hz), $59.2(\mathrm{~d}$, $\mathrm{J}=10.8 \mathrm{~Hz}), 26.4(\mathrm{~d}, \mathrm{~J}=21.5 \mathrm{~Hz}), 25.2(\mathrm{~d}, \mathrm{~J}=14.4 \mathrm{~Hz}), 18.0(\mathrm{~d}, \mathrm{~J}=3.4 \mathrm{~Hz}), 17.5 .{ }^{31} \mathrm{P} \mathrm{NMR}(162 \mathrm{MHz}$, $\left.\mathrm{C}_{6} \mathrm{D}_{6}\right) \delta 123.76(\mathrm{~s})$.

ATR-FTIR (solid, $\left.\mathrm{cm}^{-1}\right): v(\mathrm{CO})=1812,1882$

HRMS (ESI) calcd. for $\mathrm{C}_{16} \mathrm{H}_{24} \mathrm{MnN}_{2} \mathrm{O}_{2} \mathrm{P}[\mathrm{M}+\mathrm{H}]^{+}:$363.1029; found: 363.1037.

\section{Amido Complex [Mn]-4a}

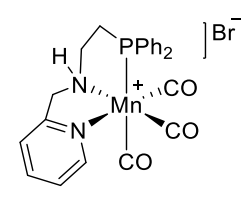

$[\mathrm{Mn}]-4$

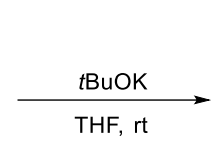

$\mathrm{HF}, \mathrm{rt}$

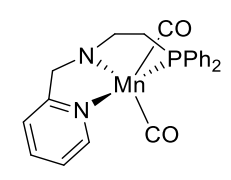

$[\mathrm{Mn}]-\mathbf{4 a}$

A dried $25 \mathrm{~mL}$ Schlenk flask was charged with [Mn]-4 (108 mg, $0.2 \mathrm{mmol})$, potassium tert-butoxide (25 $\mathrm{mg}, 0.22 \mathrm{mmol})$ and THF (4 mL) in sequence. The solution soon turned into deep red. The reaction was stirred for 1 hour. After the volatiles were removed under reduced pressure, benzene $(10 \mathrm{~mL})$ was added and the resulted solution was filtered to remove unsolved salts. After that, the benzene solution was concentrated to dryness followed by the addition of hexane $(10 \mathrm{~mL})$ and then the suspension was filtered. The red solid precipitate was collected and dried to give [Mn]-4a (62 mg, 72\%).

${ }^{1} \mathrm{H}$ NMR (400 MHz, $\left.\mathrm{C}_{6} \mathrm{D}_{6}\right) \delta 9.32(\mathrm{~s}, 1 \mathrm{H}), 7.72(\mathrm{t}, J=8.6 \mathrm{~Hz}, 4 \mathrm{H}), 7.07(\mathrm{~m}, 6 \mathrm{H}), 6.70(\mathrm{~s}, 1 \mathrm{H}), 6.46$ (s, $1 \mathrm{H}), 6.30(\mathrm{~s}, 1 \mathrm{H}), 3.82(\mathrm{~s}, 2 \mathrm{H}), 3.29(\mathrm{~d}, J=16.3 \mathrm{~Hz}, 2 \mathrm{H}), 2.54(\mathrm{t}, J=6.8 \mathrm{~Hz}, 2 \mathrm{H}) .{ }^{13} \mathrm{C} \mathrm{NMR}(101$ $\left.\mathrm{MHz}, \mathrm{C}_{6} \mathrm{D}_{6}\right) \delta 166.0,154.7,136.9(\mathrm{~d}, J=36.8 \mathrm{~Hz}), 133.7,132.2(\mathrm{~d}, J=10.3 \mathrm{~Hz}), 129.3,120.9,119.0$, $69.4(\mathrm{~d}, J=6.2 \mathrm{~Hz}), 56.2(\mathrm{~d}, J=11.9 \mathrm{~Hz}), 35.7(\mathrm{~d}, J=21.4 \mathrm{~Hz}) .{ }^{31} \mathrm{P} \mathrm{NMR}\left(162 \mathrm{MHz}, \mathrm{C}_{6} \mathrm{D}_{6}\right) \delta 103.48$ (s).

Elemental analysis calcd (\%) for: C, 61.41; H, 4.68; N, 6.51; found: C, 62.32; H, 5.05; N, 6.63

ATR-FTIR (solid, $\left.\mathrm{cm}^{-1}\right): v(\mathrm{CO})=1820,1894$

HRMS (ESI) calcd. for $\mathrm{C}_{22} \mathrm{H}_{20} \mathrm{MnN}_{2} \mathrm{O}_{2} \mathrm{P}[\mathrm{M}+\mathrm{H}]^{+}$: 431.016 ; found: 431.0724 .

Single crystal suitable for X-ray diffraction measurements were crystallized from diethyl ether solution at $-35{ }^{\circ} \mathrm{C}$ (Figure $\mathrm{S} 2$ ). 


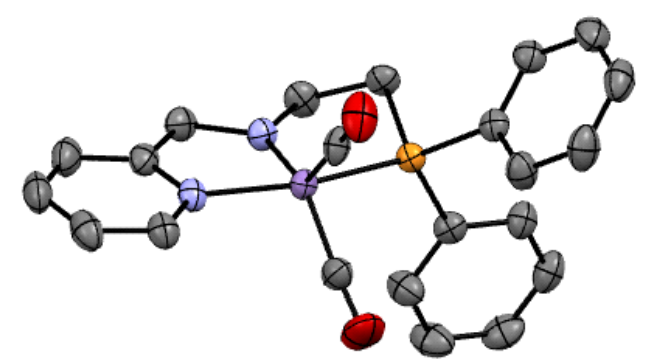

Figure S2. Crystal structure of complex [Mn]-4a

\section{Amido Complex [Mn]-6a}

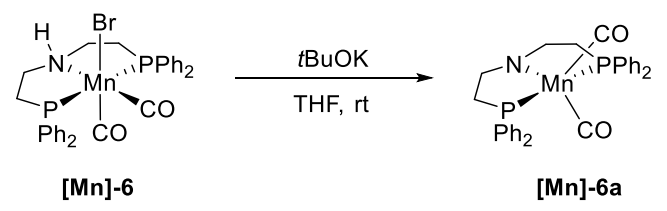

A dried $25 \mathrm{~mL}$ Schlenk flask was charged with [Mn]-6 (100 mg, $0.158 \mathrm{mmol})$, potassium tert-butoxide (19 mg, $0.17 \mathrm{mmol})$ and THF (4 mL) in sequence. The solution soon turned into dark red. The reaction was stirred for 1 hour. After the volatiles were removed under reduced pressure, benzene $(10 \mathrm{~mL})$ was added and the resulted solution was filtered to remove unsolved salts. After that, the benzene solution was concentrated to dryness followed by the addition of hexane $(10 \mathrm{~mL})$ and then the suspension was filtered. The shiny red solid precipitate was collected and dried to give [Mn]-6a (35 mg, 40\%).

${ }^{1} \mathrm{H}$ NMR (400 MHz, $\left.\mathrm{C}_{6} \mathrm{D}_{6}\right) \delta 7.84-7.62(\mathrm{~m}, 8 \mathrm{H}), 7.13-7.00(\mathrm{~m}, 12 \mathrm{H}), 3.34-3.16(\mathrm{~m}, 4 \mathrm{H}), 2.41-$ 2.25 (m, $4 \mathrm{H}) .{ }^{31} \mathrm{P}$ NMR (162 MHz, $\left.\mathrm{C}_{6} \mathrm{D}_{6}\right) \delta 91.17(\mathrm{~s})$.

ATR-FTIR (solid, $\left.\mathrm{cm}^{-1}\right): v(\mathrm{CO})=1832,1905$

HRMS (ESI) calcd. for $\mathrm{C}_{30} \mathrm{H}_{28} \mathrm{MnNO}_{2} \mathrm{P}_{2}[\mathrm{M}+\mathrm{H}]^{+}:$552.1049; found: 552.1048 . 
Reaction of Amido Mn Complexes [Mn]-1a with $\mathrm{H}_{2}$

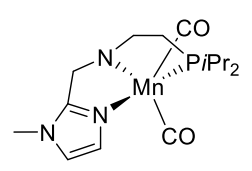

$[\mathrm{Mn}]-1 \mathrm{a}$

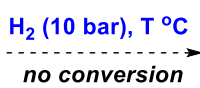

no conversion

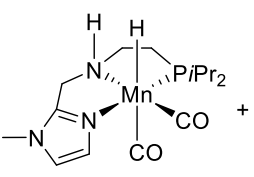

$[\mathrm{Mn}]-\mathbf{1 b}$

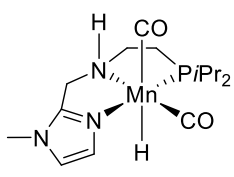

[Mn]-1b'

In a high-pressure NMR tube, complex [Mn]-1a $(3.7 \mathrm{mg}, 0.01 \mathrm{~mol})$ were dissolved in $0.2 \mathrm{~mL}$ THF-d8 solvent. Then $\mathrm{H}_{2}$ (10 bar) were added to the high-pressure NMR tube. The tube was placed into NMR apparatus and in suit heated, then ${ }^{1} \mathrm{H}$ NMR was collected at $60{ }^{\circ} \mathrm{C}, 80^{\circ} \mathrm{C}$, and $100{ }^{\circ} \mathrm{C}$ (hold for 30 min at $100{ }^{\circ} \mathrm{C}$ ). No hydride signals were observed (Figure S3).

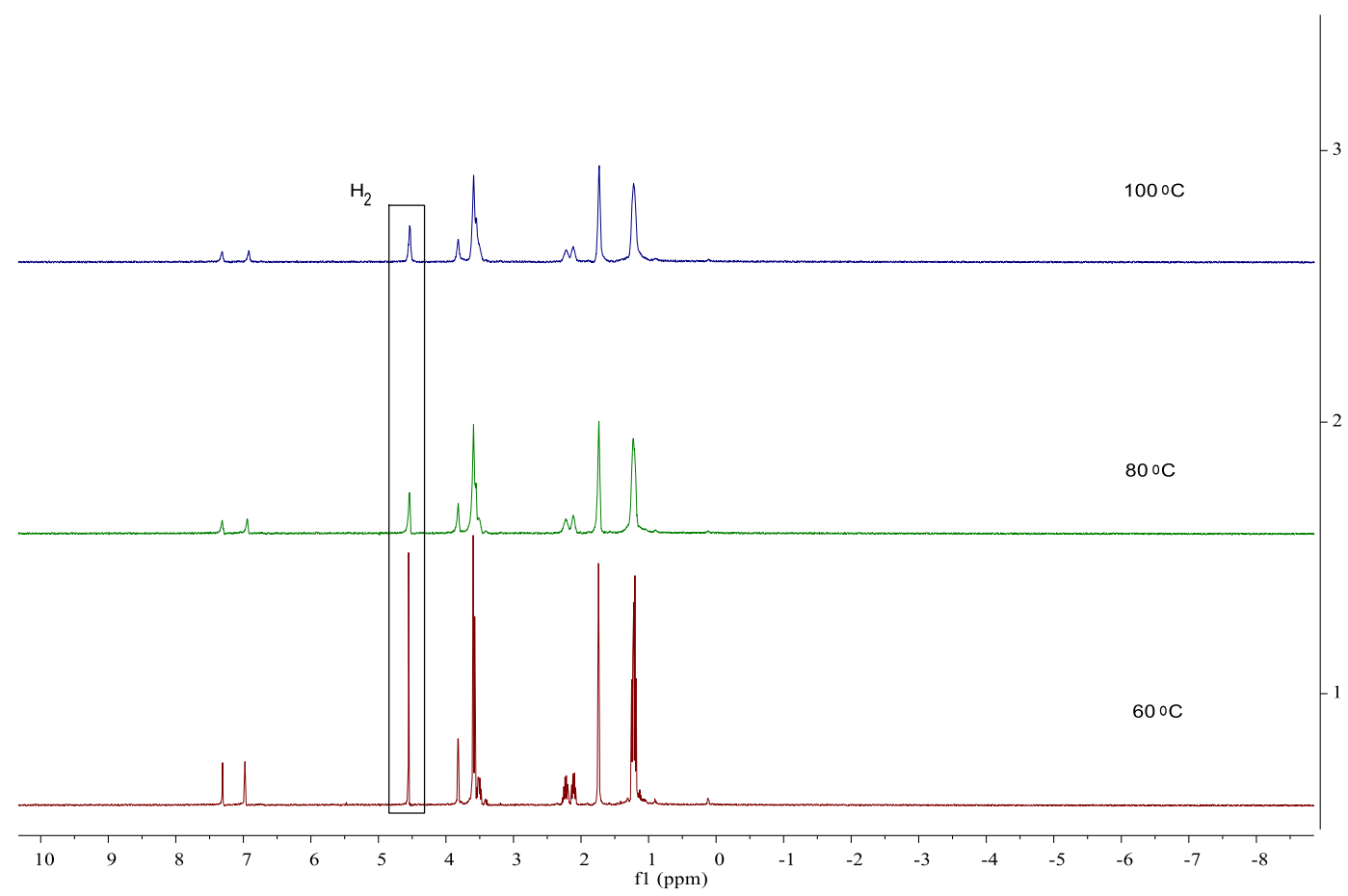

Figure S3. ${ }^{1} \mathrm{H}$ NMR $\left(400 \mathrm{MHz}, \mathrm{THF}-\mathrm{d}_{8}\right)$ of reacting [Mn]-1a with $\mathrm{H}_{2}(10$ bar) at different temperature

Kinetic Studies: Reaction of 1,2-Dihydroquinoline 7a with Amido Mn Complexes [Mn]-1a

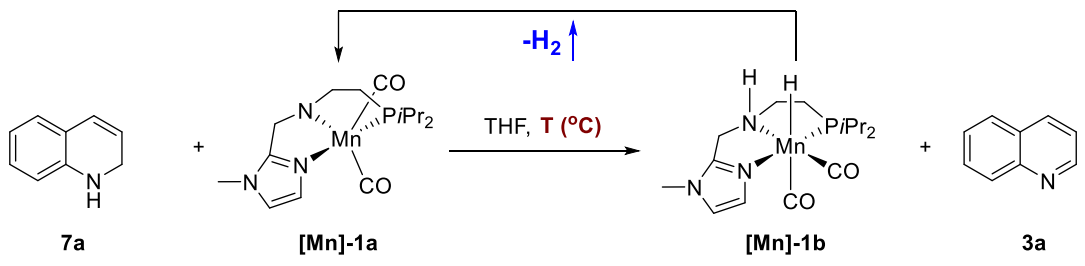

In NMR tube, 1,2-dihydroquinoline 7a $(13 \mathrm{mg}, 0.1 \mathrm{mmol})$ and complex [Mn]-1a $(1.8 \mathrm{mg}, 0.005 \mathrm{~mol})$ were dissolved in $0.4 \mathrm{~mL}$ of $\mathrm{THF}-\mathrm{d}_{8}$ solvent. Then the tube was placed without perturbation at room temperature. ${ }^{1} \mathrm{H}$ NMR of this reaction mixture was collected at different time interval. As shown in Figure S4, the content of 1,2-dihydroquinoline 7a was decreased and quinoline 3a was increased over time. Dihydrogen was clearly observed in the reaction system. ${ }^{7}$ 


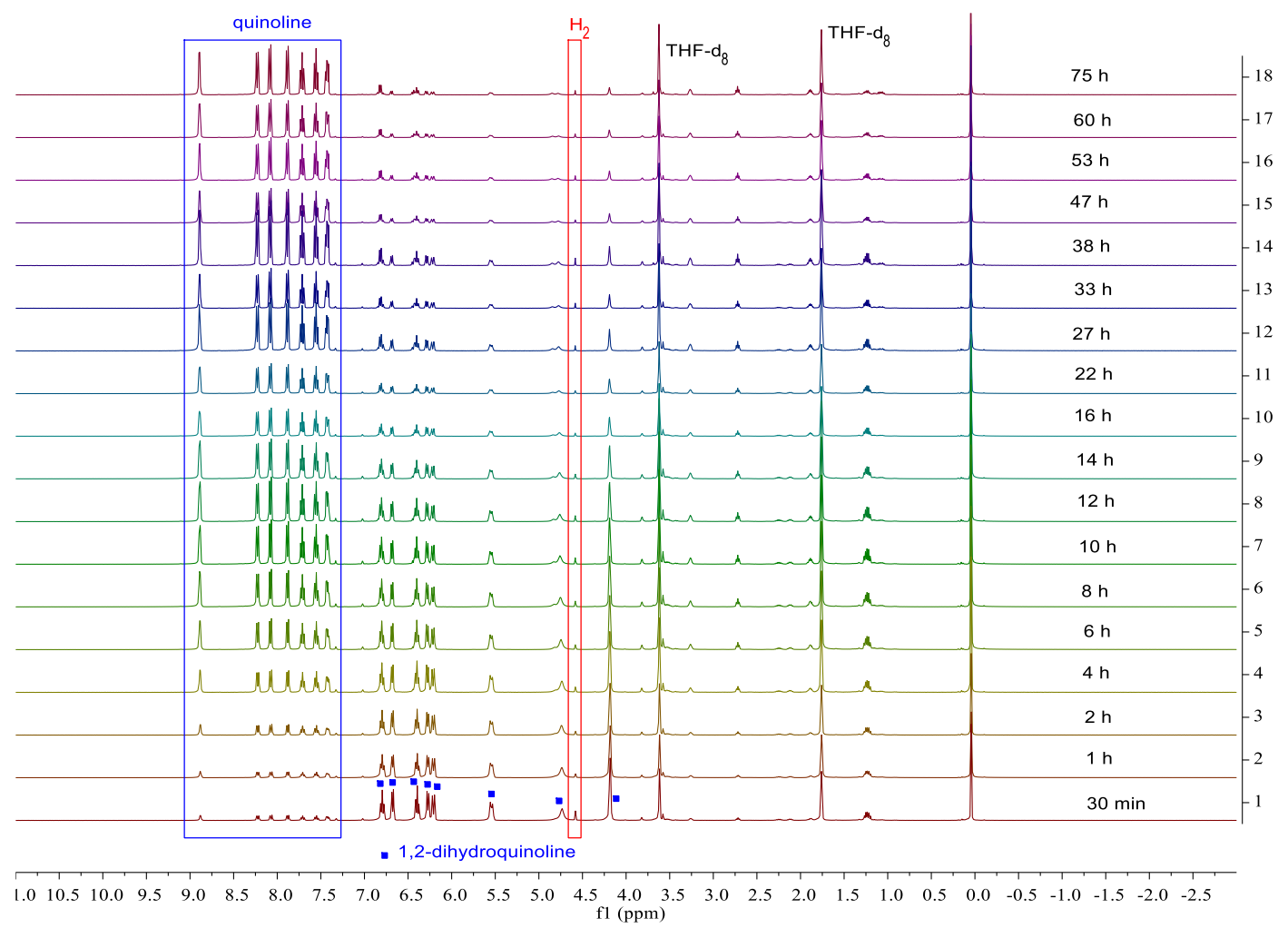

Figure S4. ${ }^{1} \mathrm{H}$ NMR $\left(400 \mathrm{MHz}, \mathrm{THF}-\mathrm{d}_{8}\right)$ of reacting $7 \mathbf{a}$ with $[\mathbf{M n}]-\mathbf{1 a}$ at $25{ }^{\circ} \mathrm{C}$

${ }^{1} \mathrm{H}$ NMR of reaction mixture performed at $0{ }^{\circ} \mathrm{C}\left(\mathrm{THF}-\mathrm{d}_{0}\right)$ was shown in Figure S5.

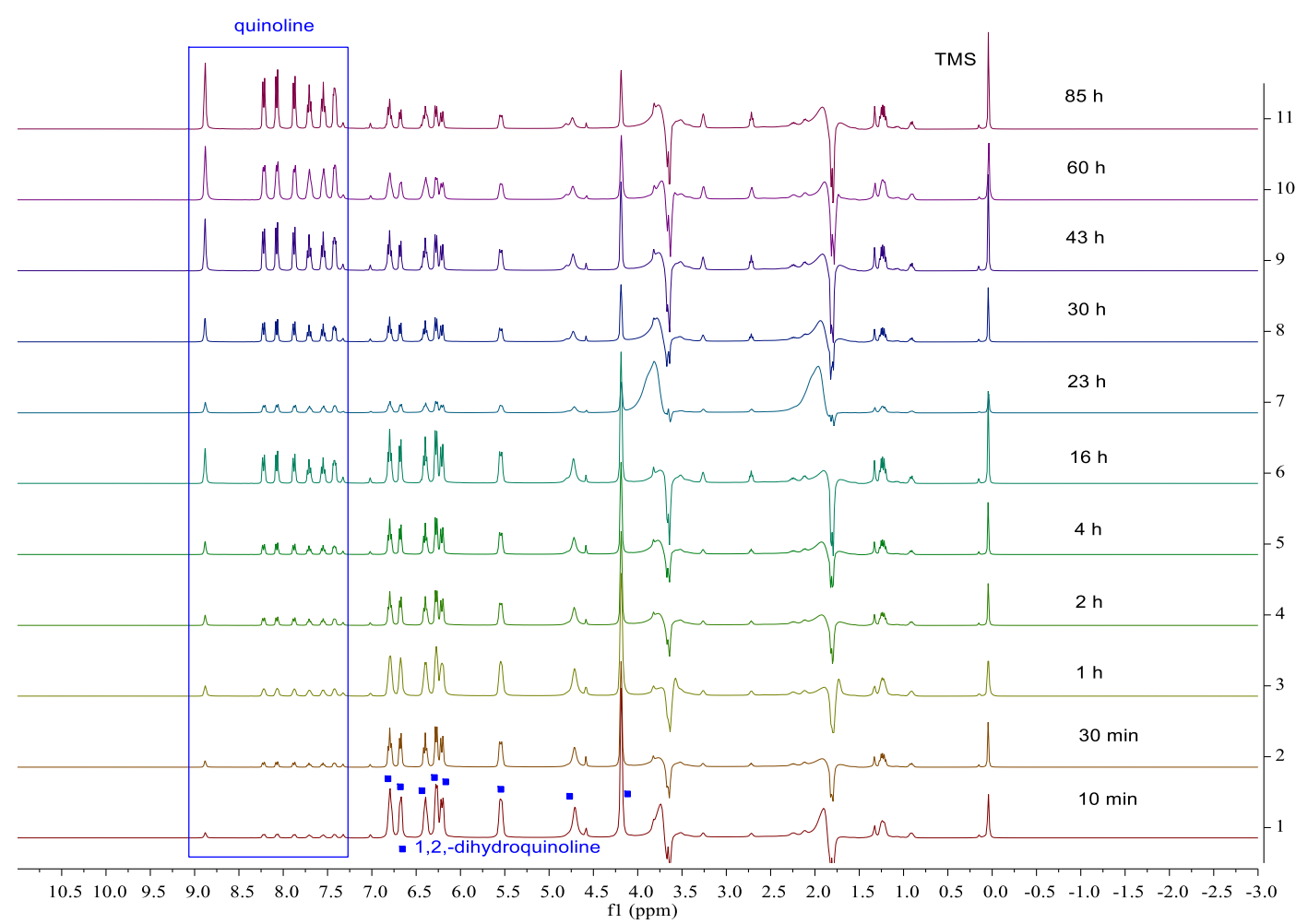

Figure S5. ${ }^{1} \mathrm{H}$ NMR $\left(400 \mathrm{MHz}, \mathrm{THF}-\mathrm{d}_{0}\right)$ of reacting $7 \mathbf{a}$ with $[\mathbf{M n}]-\mathbf{- 1 a}$ at $0{ }^{\circ} \mathrm{C}$ 
${ }^{1} \mathrm{H}$ NMR of reaction mixture performed at $-35^{\circ} \mathrm{C}\left(\mathrm{THF}-\mathrm{d}_{0}\right)$ was shown in Figure S6.

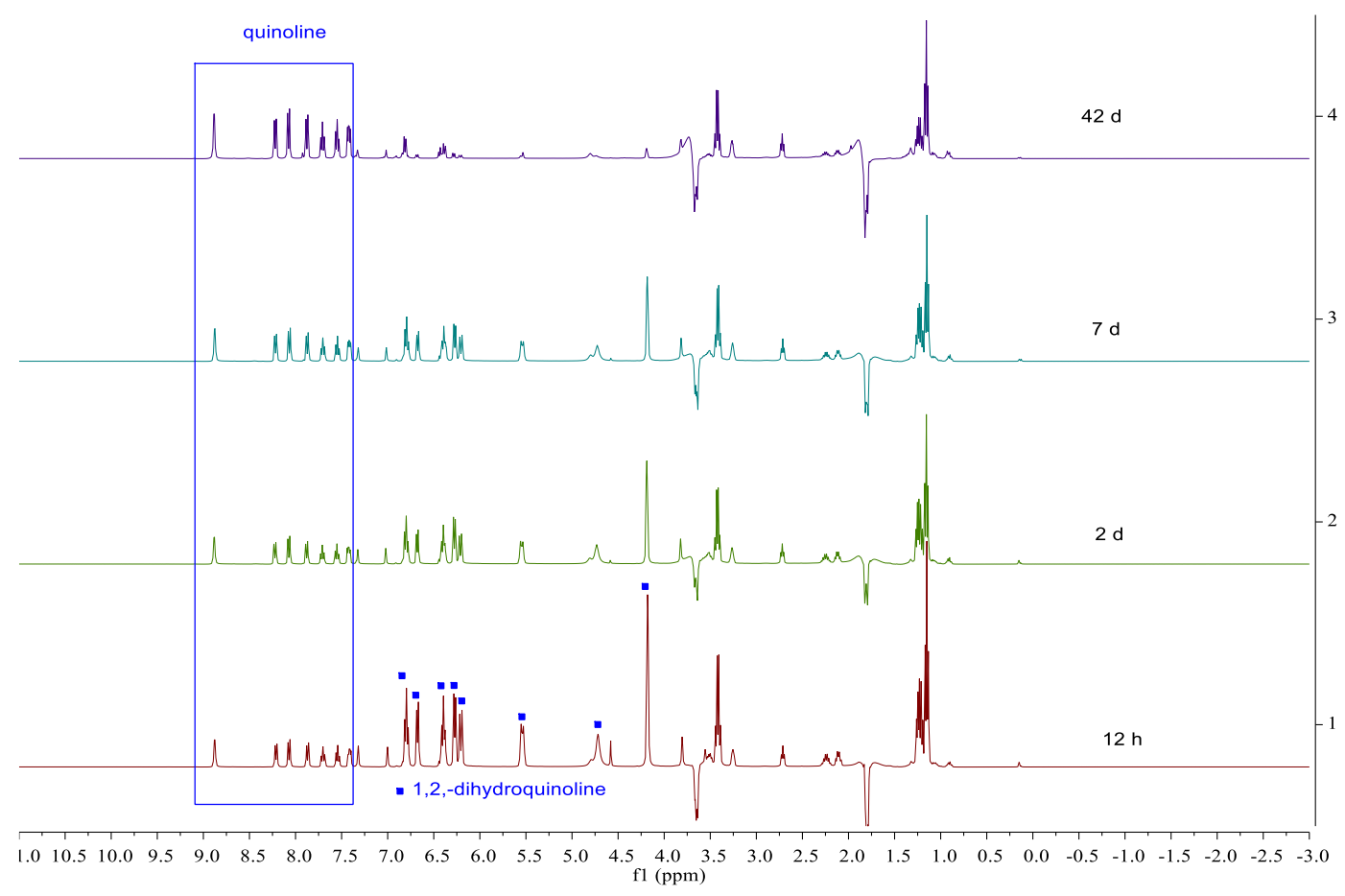

Figure S6a. ${ }^{1} \mathrm{H}$ NMR (400 MHz, THF-d $\left.\mathrm{d}_{0}\right)$ of reacting 7a with [Mn]-1a at $-35{ }^{\circ} \mathrm{C}$ (showing signals range from -3 ppm to $11 \mathrm{ppm}$ )

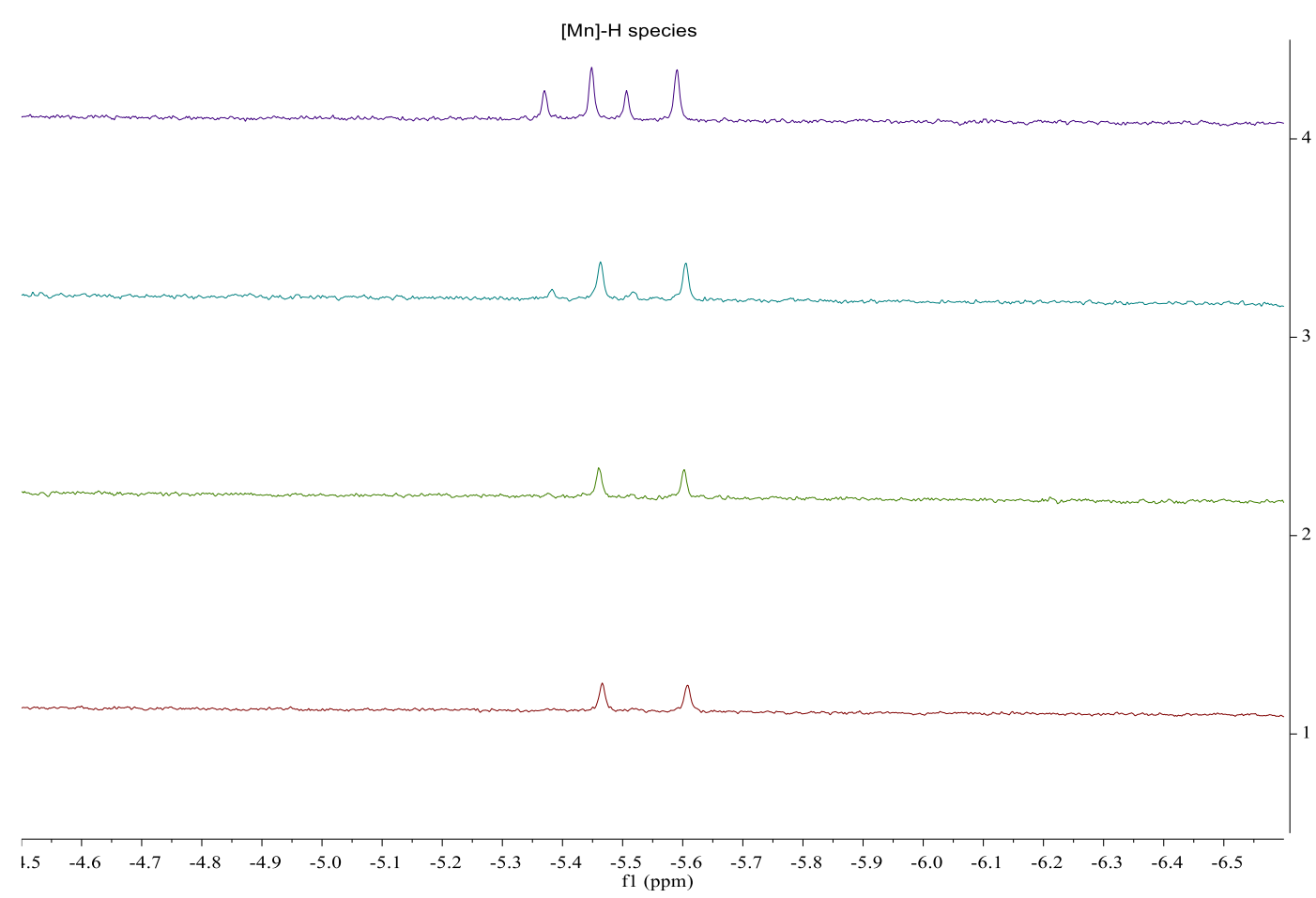

Figure S6b. ${ }^{1} \mathrm{H}$ NMR $\left(400 \mathrm{MHz}, \mathrm{THF}-\mathrm{d}_{0}\right)$ of reacting $7 \mathbf{a}$ with $[\mathbf{M n}]-\mathbf{1 a}$ at $-35{ }^{\circ} \mathrm{C}$ (showing signals range from $-6.6 \mathrm{ppm}$ to $-4.5 \mathrm{ppm})$ 


\section{Procedures for Mn-Catalyzed Hydrogenation of Methyl 4-Acetylbenzoate 2a}

Under the argon atmosphere glovebox, $t \mathrm{BuOK}(0.7 \mathrm{mg}, 2.5 \mathrm{~mol} \%)$, PNP and NNP pincer manganese complexes ( $2 \mathrm{~mol} \%), 0.5 \mathrm{~mL}$ of THF, and methyl 4-acetylbenzoate $2 \mathrm{a}$ ( $44.5 \mathrm{mg}, 0.25 \mathrm{mmol}$ ) were added sequentially to the vial with a magnetic stir bar, which was capped with a septum equipped with a syringe. The vial was placed in the alloy plate, which was then transferred into pre-dried autoclave. Once sealed, the autoclave was purged with hydrogen for three times, followed by pressurized to 45 bar of $\mathrm{H}_{2}$ and heated at $120{ }^{\circ} \mathrm{C}$ for $12 \mathrm{~h}$. After reaction, the autoclave was cooled with ice-water bath, and then depressurized. The reaction mixture was diluted by $2 \mathrm{~mL}$ of $\mathrm{CH}_{2} \mathrm{Cl}_{2}$. Diphenyl as internal standard was added to determinate yield of methyl 4-(1-hydroxyethyl)benzoate $\mathbf{2 b}$ and 1-(4(hydroxymethyl)phenyl)ethan-1-ol 2c by GC.

Methyl 4-(1-hydroxyethyl)benzoate $\mathbf{2 b}$ was isolated via column chromatography as colorless oil. ${ }^{8}{ }^{1} \mathrm{H}$ $\operatorname{NMR}\left(400 \mathrm{MHz}, \mathrm{CDCl}_{3}\right) \delta 7.98(\mathrm{~d}, \mathrm{~J}=8.0 \mathrm{~Hz}, 2 \mathrm{H}), 7.41(\mathrm{~d}, \mathrm{~J}=8.0 \mathrm{~Hz}, 2 \mathrm{H}), 4.93$ (q, J = 6.5 Hz, $1 \mathrm{H}$ ), $3.89(\mathrm{~s}, 3 \mathrm{H}), 2.28(\mathrm{~s}, 1 \mathrm{H}), 1.48(\mathrm{~d}, \mathrm{~J}=6.4 \mathrm{~Hz}, 3 \mathrm{H}) .{ }^{13} \mathrm{C} \mathrm{NMR}\left(101 \mathrm{MHz}, \mathrm{CDCl}_{3}\right) \delta 167.0,151.0,129.8$, 129.1, 125.2, 69.9, 52.0, 25.2.

1-(4-(hydroxymethyl)phenyl)ethan-1-ol 2c was isolated via column chromatography as colorless oil. ${ }^{9}{ }^{1} \mathrm{H}$ NMR (400 MHz, $\left.\mathrm{CDCl}_{3}\right) \delta$ 7.39-7.29 (m, $\left.4 \mathrm{H}\right), 4.88$ (q, J = 6.5 Hz, $\left.1 \mathrm{H}\right), 4.65$ (s, $\left.2 \mathrm{H}\right), 2.05$ (s, $2 \mathrm{H}$ ), $1.48(\mathrm{~d}, \mathrm{~J}=6.4 \mathrm{~Hz}, 3 \mathrm{H}) .{ }^{13} \mathrm{C} \mathrm{NMR}\left(101 \mathrm{MHz}, \mathrm{CDCl}_{3}\right) \delta 145.2,140.1,127.1,125.6,70.1,65.0,25.1$.

Table S2. Optimization of Reaction Parameters for [Mn]-1 Catalyzed Hydrogenation of Quinoline 3a ${ }^{a}$

$\begin{array}{llllll} & & & \\ \text { [Mn]-1 (2 mol\%) } \\ \text { base, solvent }\end{array}$

${ }^{a}$ Reaction conditions: quinoline 3a $(0.25 \mathrm{mmol})$, Mn-1 ( $\left.2 \mathrm{~mol} \%\right)$, base in solvent were reacted at $120^{\circ} \mathrm{C}$ under given $\mathrm{H}_{2}$ pressure for 16 h. ${ }^{b}$ conversion and yield determined by GC using biphenyl as internal standard. ${ }^{c}$ Reacted at $100{ }^{\circ} \mathrm{C}$. ${ }^{d} \mathrm{Mn}(\mathrm{CO})_{5} \mathrm{Br}$ used as catalyst.

General procedure: Under the argon atmosphere glovebox, $t \mathrm{BuOK},[\mathbf{M n}]-\mathbf{1}(2.4 \mathrm{mg}, 2 \mathrm{~mol} \%)$, solvent, 
and quinoline 3a (32 mg, $0.25 \mathrm{mmol}$ ) were added sequentially to the vial with a magnetic stir bar, which was capped with a septum equipped with a syringe. The vial was placed in the alloy plate, which was then transfered into a predried autoclave. Once sealed, the autoclave was purged with hydrogen for three times, followed by pressurized to given pressure and heated at $120{ }^{\circ} \mathrm{C}$ for $16 \mathrm{~h}$. After reaction, the autoclave was cooled with ice-water bath, and then depressurized. The reaction mixture was diluted by $2 \mathrm{~mL}$ of $\mathrm{CH}_{2} \mathrm{Cl}_{2}$. The conversion of $\mathbf{3 a}$ and yield of $\mathbf{4 a}$ was determined by $\mathrm{GC}$ with diphenyl as the internal standard.

Table S3. Homogenity Tests for [Mn]-1 Catalyzed Hydrogenation of Quinoline 3a ${ }^{a}$

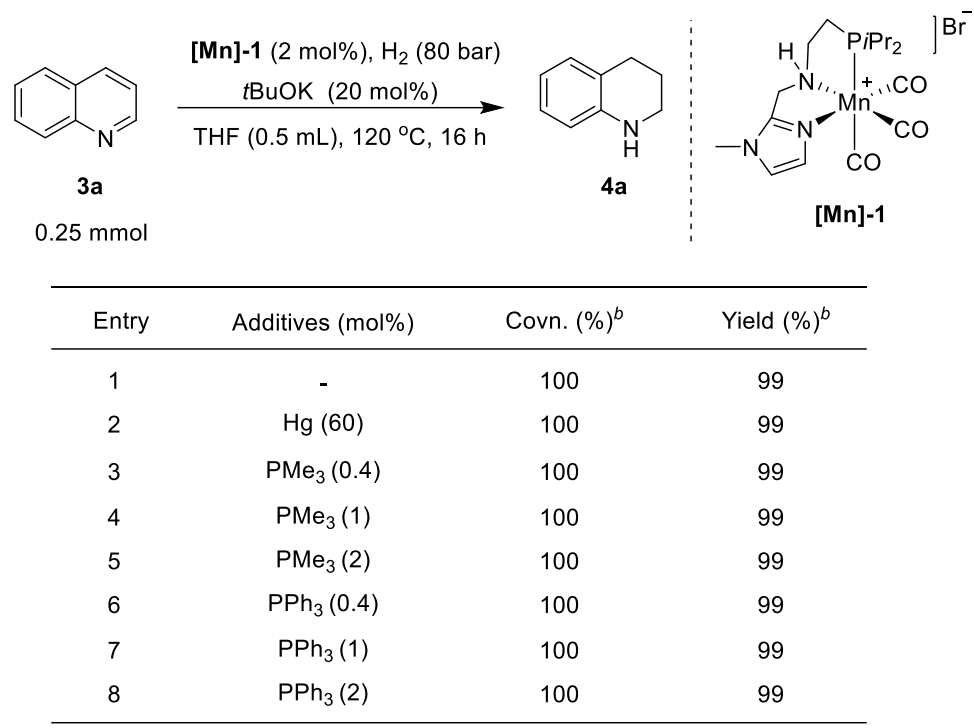

${ }^{a}$ Reaction conditions: Quinoline 3a (0.25 mmol), [Mn]-1 (2 mol\%), $t \mathrm{BuOK}(20 \mathrm{~mol} \%)$, THF $(0.5 \mathrm{~mL})$, additives (phosphines or mercury), $120{ }^{\circ} \mathrm{C}, \mathrm{H}_{2}$ (80 bar), 16 h. ${ }^{b}$ conversion and yield determined by $\mathrm{GC}$ using biphenyl as internal standard.

Under the argon atmosphere glovebox, $t \mathrm{BuOK}$ (5.6 mg, $0.05 \mathrm{mmol}, 20 \mathrm{~mol} \%)$, [Mn]-1 (2.4 mg, 0.005 mmol, $2 \mathrm{~mol} \%)$, THF (0.5 mL), quinoline 3a (32 mg, $0.25 \mathrm{mmol})$ and varying amounts of poisoning agents $\left(\mathrm{Hg}, \mathrm{PMe}_{3}, \mathrm{PPh}_{3}\right)$ were added sequentially to the vial with a magnetic stir bar, which was capped with a septum equipped with a syringe. The vial was placed in the alloy plate, which was then transfered into a predried autoclave. Once sealed, the autoclave was purged with hydrogen for three times, followed by pressurized to 80 bar of $\mathrm{H}_{2}$ and heated at $120{ }^{\circ} \mathrm{C}$ for $16 \mathrm{~h}$. After reaction, the autoclave was cooled with ice-water bath, and then depressurized. The reaction mixture was diluted by $2 \mathrm{~mL}$ of $\mathrm{CH}_{2} \mathrm{Cl}_{2}$. The conversion of $\mathbf{3 a}$ and yield of $\mathbf{4 a}$ was determined by GC with diphenyl as the internal standard.

\section{General Procedure for the Mn-Catalyzed Hydrogenation of $N$-Heterocycles}

All hydrogenation experiments were carried out in a Parr Instruments 4560 series autoclave (300 mL) containing an alloy plate with wells for seven $4 \mathrm{~mL}$ glass vials. Under the argon atmosphere glovebox, $t \mathrm{BuOK}$ (5.6 mg, $0.05 \mathrm{mmol}, 20 \mathrm{~mol} \%)$, [Mn]-1 or [Mn]-4 (0.005 mmol, $2 \mathrm{~mol} \%)$, THF (0.5 mL), and $N$-heterocycles $(0.25 \mathrm{mmol})$ were added sequentially to the vial with a magnetic stir bar, which was capped with a septum equipped with a syringe. The vial was placed in the alloy plate, which was then transfered into a predried autoclave. Once sealed, the autoclave was purged with hydrogen for three times, 
followed by pressurized to 80 bar of $\mathrm{H}_{2}$ and heated at $120^{\circ} \mathrm{C}$ for $16 \mathrm{~h}$. After reaction, the autoclave was cooled with ice-water bath, and then depressurized. The products was isolated by a flash column chromatography.

\section{Synthesis of 5,10-diethyl-1,2,3,4,4a,5,10,10a-octahydrophenazine $6 \mathrm{n}$}

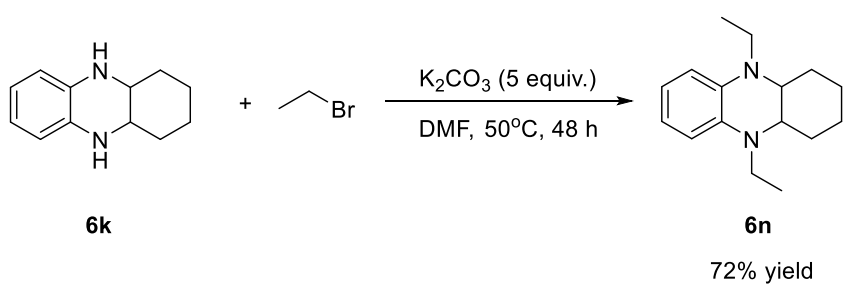

A mixture of 1,2,3,4,4a,5,10,10a-octahydro-phenazine $6 \mathbf{k}(47 \mathrm{mg}, 0.25 \mathrm{mmol})$, potassium carbonate (172 $\mathrm{mg}, 1.25 \mathrm{mmol}$ ) and bromoethane $(648 \mathrm{mg}, 1.5 \mathrm{mmol})$ in $2 \mathrm{~mL}$ of DMF is stirred at $50{ }^{\circ} \mathrm{C}$ for 48 hours. After reaction, the reaction mixture is filtered, concertrated and then purified via chromatography on silica gel to yield 5,10-diethyl-1,2,3,4,4a,5,10,10a-octahydrophenazine $\mathbf{6 n}^{\mathbf{1 0}}$ as a yellow oil (44 mg, $72 \%$ yield).

${ }^{1} \mathrm{H}$ NMR (400 MHz, $\left.\mathrm{CDCl}_{3}\right) \delta$ 6.70-6.56 (m, $\left.4 \mathrm{H}\right), 3.45(\mathrm{tt}, J=12.6,6.3 \mathrm{~Hz}, 2 \mathrm{H}), 3.36-3.28(\mathrm{~m}, 2 \mathrm{H})$, 3.21 (dq, $J=14.4,7.1 \mathrm{~Hz}, 2 \mathrm{H}), 1.89$ (td, $J=10.8,9.8,4.7 \mathrm{~Hz}, 2 \mathrm{H}), 1.69-1.47$ (m, $4 \mathrm{H}), 1.42-1.34$ (m, $2 \mathrm{H}), 1.17(\mathrm{t}, J=7.0 \mathrm{~Hz}, 6 \mathrm{H}) .{ }^{13} \mathrm{C} \mathrm{NMR}\left(101 \mathrm{MHz}, \mathrm{CDCl}_{3}\right) \delta 135.0,117.2,111.1,55.1,42.0,27.6,22.4$, 11.8 .

\section{Synthesis of 1,2-Dihydroquinoline 7a}

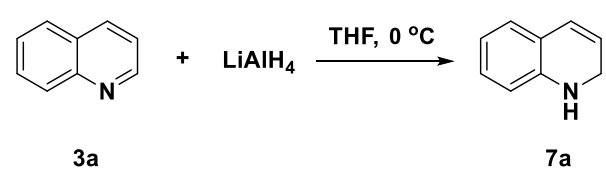

In a $250 \mathrm{~mL}$ Schlenk flask, a solution of quinoline 3a $(5.0 \mathrm{~g}, 38.8 \mathrm{mmol})$ in $100 \mathrm{~mL}$ of anhydrous THF was added lithium aluminium hydride $(1.72 \mathrm{~g}, 46.5 \mathrm{mmol})$ in small portions at $0{ }^{\circ} \mathrm{C}$. The reaction was stirred at $0{ }^{\circ} \mathrm{C}$ for $2 \mathrm{~h}$ and then cold water $(100 \mathrm{~mL})$ was cautiously added with continued stirring. The layers were separated, the aqueous layer was extracted with ether $(3 \times 50 \mathrm{~mL})$ and the combined organic layers were dried with $\mathrm{MgSO}_{4}$ and concentrated in vacuo. The residue was purified by flash chromatography to give crude $\mathbf{7 a}$ as a pale yellow solid. Then, then crude product was dissolved with $\operatorname{DCM}(5 \mathrm{~mL})$ and then petroleum ether was carefully added $(100 \mathrm{~mL})$, which was placed into refrigerator at $-35^{\circ} \mathrm{C}$. After 2 days, product $7 \mathbf{a}$ was recrystallized as white solid $(3.0 \mathrm{~g}, 60 \%) .{ }^{11} \mathbf{7 a}$ was sensitive to air but could be stored without obvious decomposing at $-35^{\circ} \mathrm{C}$ under Ar.

${ }^{1} \mathrm{H}$ NMR $\left(400 \mathrm{MHz}, \mathrm{CDCl}_{3}\right) \delta 6.93(\mathrm{td}, J=7.6,1.6 \mathrm{~Hz}, 1 \mathrm{H}), 6.85-6.77(\mathrm{~m}, 1 \mathrm{H}), 6.58(\mathrm{t}, J=7.4 \mathrm{~Hz}, 1$ H), $6.36(\mathrm{~d}, J=7.9 \mathrm{~Hz}, 1 \mathrm{H}), 6.29(\mathrm{dd}, J=9.9,2.1 \mathrm{~Hz}, 1 \mathrm{H}), 5.65(\mathrm{dt}, J=9.9,3.9 \mathrm{~Hz}, 1 \mathrm{H}), 4.19(\mathrm{dd}, J$ = 4.1, 2.0 Hz, $2 \mathrm{H}), 3.60$ (br, $1 \mathrm{H}) .{ }^{13} \mathrm{C} \mathrm{NMR}\left(101 \mathrm{MHz}, \mathrm{CDCl}_{3}\right) \delta 144.8,128.6,126.7,126.2,121.9$, 121.0, 117.7, 112.5, 43.1 . 


\section{Procedures for [Mn]-1a Catalyzed Hydrogenation of Quinoline 3a}

Under the argon atmosphere glovebox, $t \mathrm{BuOK}(1.4$ or $0 \mathrm{mg}, 5$ or $0 \mathrm{~mol} \%)$, [Mn]-1a (1.8 $\mathrm{mg}, 2 \mathrm{~mol} \%)$, $0.5 \mathrm{~mL}$ of THF, and quinoline $\mathbf{3 a}(32 \mathrm{mg}, 0.25 \mathrm{mmol})$ were added sequentially to a vial with a magnetic stir bar, which was capped with a septum equipped with a syringe. The vial was placed in the alloy plate, which was then transfered into a pre-dried autoclave. Once sealed, the autoclave was purged with hydrogen for three times, followed by pressurized to 60 bar of $\mathrm{H}_{2}$ and heated at $120{ }^{\circ} \mathrm{C}$ for different reaction time. After reaction, the autoclave was cooled with ice-water bath, and then depressurized. The reaction mixture was submitted to NMR and GC analysis. 1,3,5-trimethoxybenzene as the internal standard for NMR measurement was added to determinate the yield of 1,2-dihydroquinoline. Diphenyl as the internal standard for GC measurement was added to determinate the yield of 1,2,3,4tetrahydroquinoline.

With the use of base, reaction intermediate 1,2-dihydroquinoline was not observed during the reaction process (Figure S7).

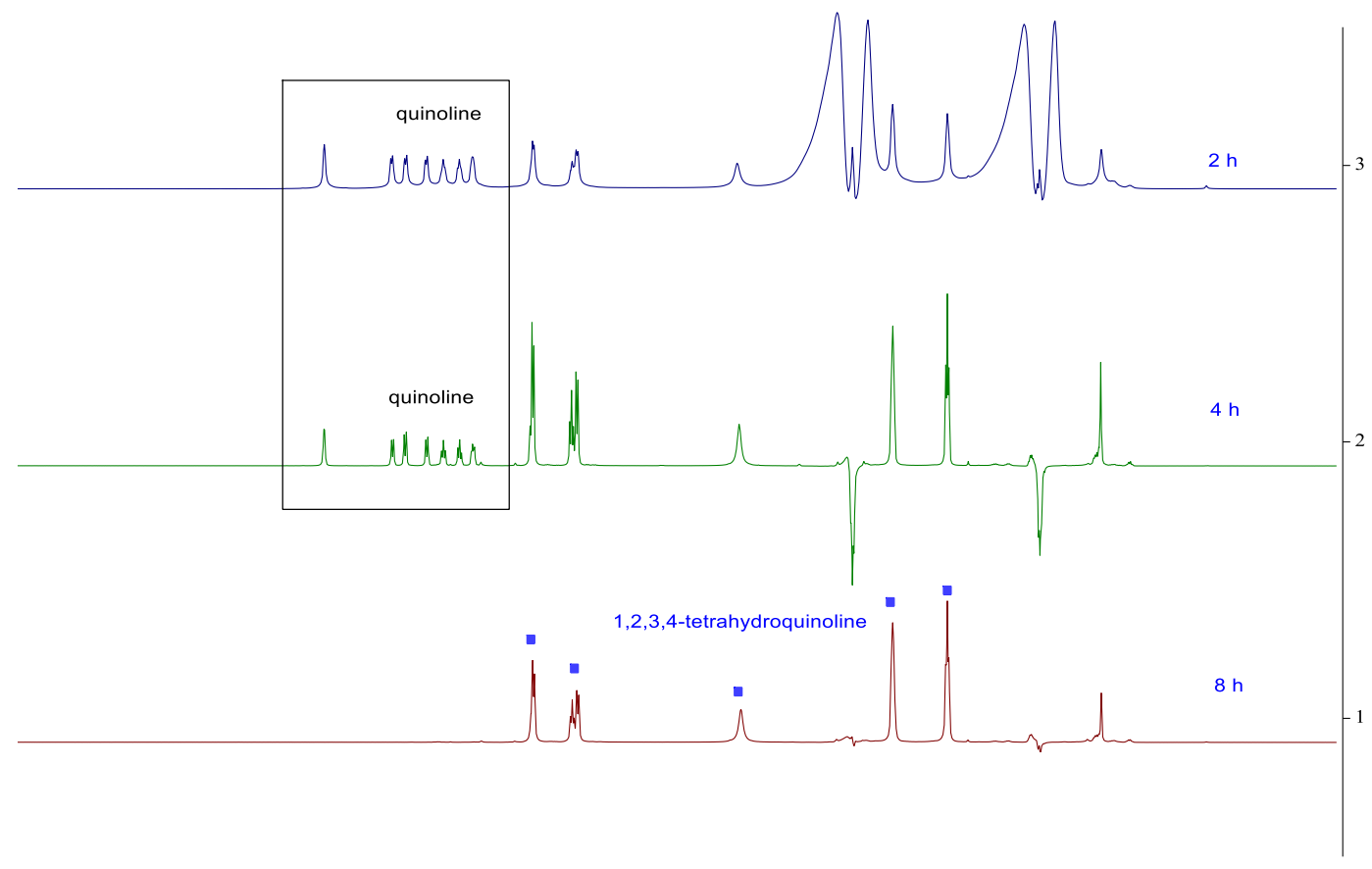

$\begin{array}{lllllllllllllllllllllllllll}11.5 & 11.0 & 10.5 & 10.0 & 9.5 & 9.0 & 8.5 & 8.0 & 7.5 & 7.0 & 6.5 & 6.0 & \begin{array}{c}5.5 \\ \mathrm{f} 1(\mathrm{ppm})\end{array} & 5.0 & 4.0 & 3.5 & 3.0 & 2.5 & 2.0 & 1.5 & 1.0 & 0.5 & 0.0 & -0.5 & -1.0\end{array}$

Figure S7. ${ }^{1} \mathrm{H}$ NMR $\left(400 \mathrm{MHz}, \mathrm{THF}-\mathrm{d}_{0}\right)$ of the reaction mixture for different reaction times with the use of $t \mathrm{BuOK}$

Without the use of base, conversion of quinoline 3a became much slower, and reaction intermediate 1,2dihydroquinoline was clearly observed on ${ }^{1} \mathrm{H}$ NMR spectra (Figure S8). 


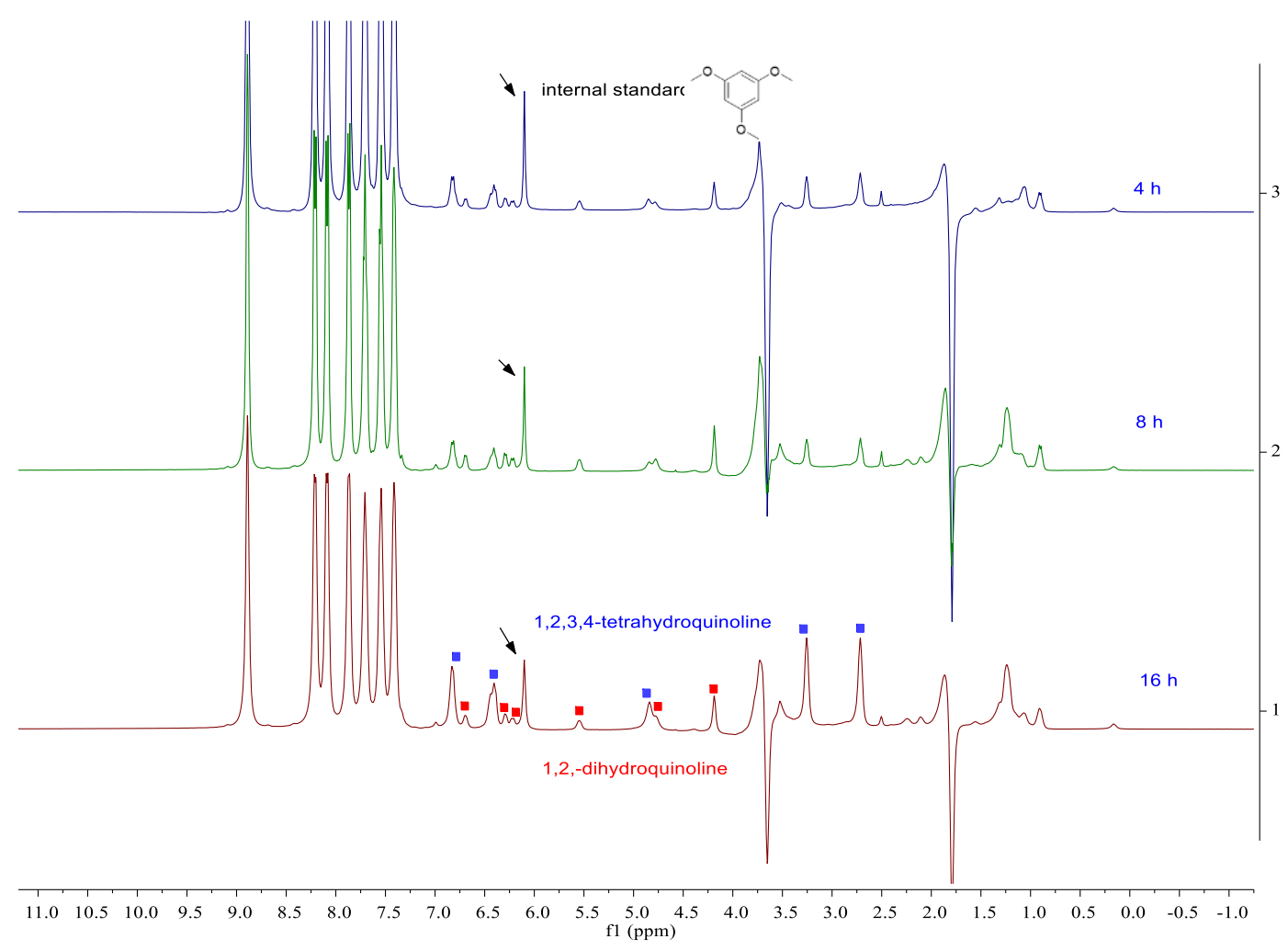

Figure S8. ${ }^{1} \mathrm{H}$ NMR $\left(400 \mathrm{MHz}, \mathrm{THF}-\mathrm{d}_{0}\right)$ of the reaction mixture for different reaction times without the use of $t \mathrm{BuOK}$

\section{Procedures for Mn-Catalyzed Hydrogenation of 1,2-Dihydroquinoline 7a}

Under the argon atmosphere glovebox, $t \mathrm{BuOK}(20,5$ or $0 \mathrm{~mol} \%)$, [Mn]-1 or [Mn]-1a $(2 \mathrm{~mol} \%), 0.5 \mathrm{~mL}$ of THF, and 1,2-dihydroquinoline $7 \mathbf{a}(33 \mathrm{mg}, 0.25 \mathrm{mmol})$ were added sequentially to the vial with a magnetic stir bar, which was capped with a septum equipped with a syringe. The vial was placed in the alloy plate, which was then transfered into a pre-dried autoclave. Once sealed, the autoclave was purged with hydrogen for three times, followed by pressurized to 60 bar of $\mathrm{H}_{2}$ and heated at $120{ }^{\circ} \mathrm{C}$ for $16 \mathrm{~h}$. After reaction, the autoclave was cooled with ice-water bath, and then depressurized. The reaction mixture was submitted to NMR and GC analysis. 1,3,5-trimethoxybenzene as the internal standard for NMR measurement was added to determinate the conversion of 1,2-dihydroquinoline. Diphenyl as the internal standard for GC measurement was added to determinate yield of 1,2,3,4-tetrahydroquinoline and quinoline.

\section{Reaction of $t \mathrm{BuOK}$ with 1,2-Dihydroquinoline 7a: Experimental Evidence of Isomerization of $7 \mathbf{a}$}

Procedures: Under the argon atmosphere glovebox, 1,2-dihydroquinoline 7a (262 mg, $2 \mathrm{mmol})$ and quinoline 3a (2.6 mg, $1 \mathrm{~mol} \%$ ), $t \mathrm{BuOK}$ (45 mg, $20 \mathrm{~mol} \%$ ), and $3 \mathrm{~mL}$ of THF were added sequentially to a $15 \mathrm{~mL}$ sealed tube with a magnetic stir bar. The reaction tube was place into pre-heated oil bath of 120 ${ }^{\circ} \mathrm{C}$ and reacted for $12 \mathrm{~h}$. After reaction, the tube was cooled down to room temperature and diphenyl as the internal standard for GC measurement was added to determinate the yield of quinoline and 1,2,3,4tetrahydroquinoline $\left(\right.$ Yield $\left._{3 a}=2 * n_{3 a} / n_{7 a}, Y_{i e l d} a=2 * n_{4 a} / n_{7 a}\right)$. Dimer products 16 and 17 were isolated by column chromatography $\left(\right.$ Yield $_{16}=2 * n_{16} / n_{7 a}$, Yield $\left.17=2 * n_{17} / n_{7 a}\right)$. 
Scheme S1. Base-Promoted Conversion of 1,2-Dihydroquinoline 7a
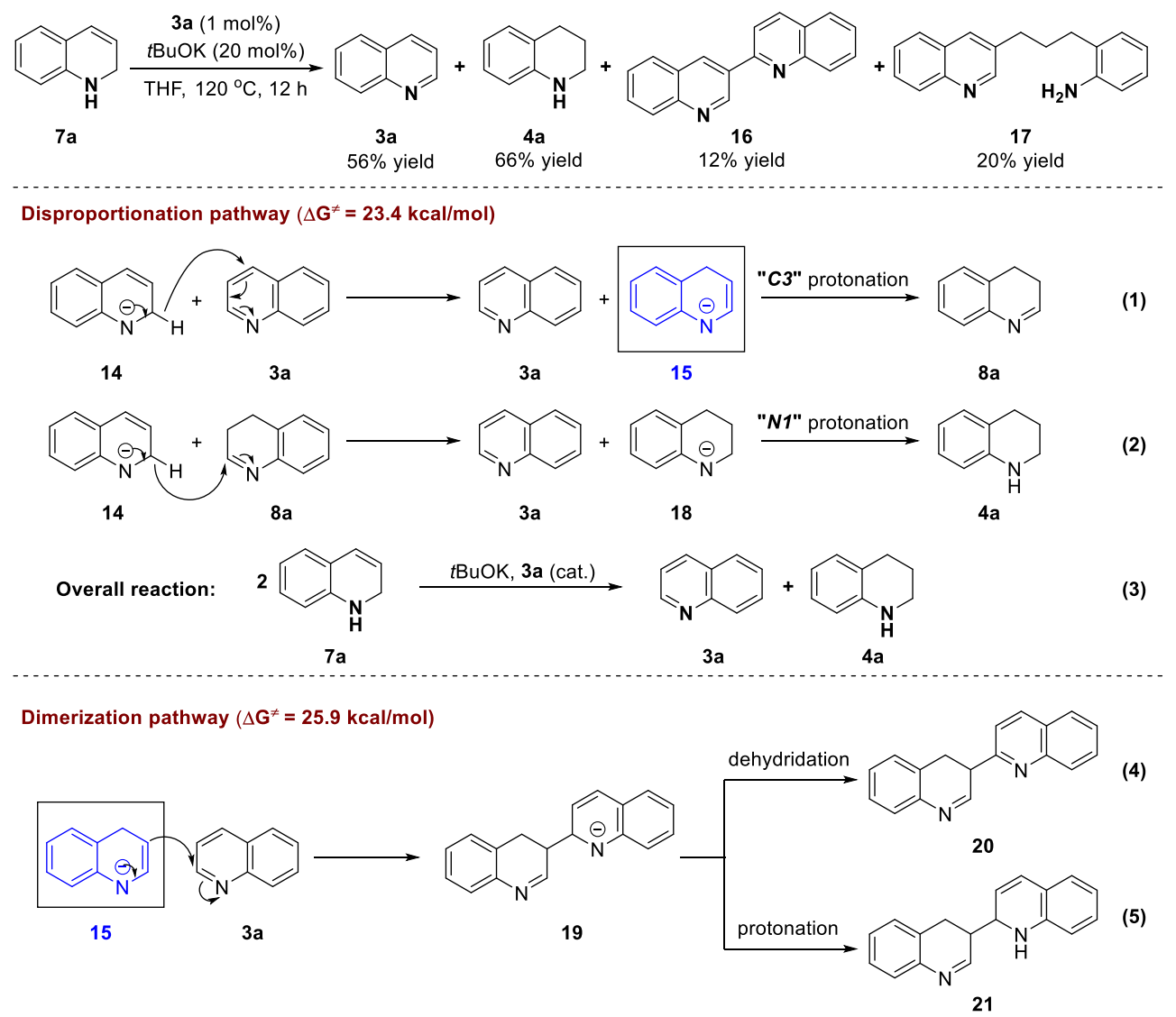

The reaction of 1,2-dihydroquinoline (7a) with $t \mathrm{BuOK}$ was conducted to obtain experimental evidence of the isomerization process. Interestingly, 7a was mainly converted into quinoline (3a) in 56\% yield and 1,2,3,4-tetrahydroquinoline (4a) in 66\% yield rather than isomerized products $8 \mathbf{a}$ or $\mathbf{9 a}$ (Scheme S8). The disproportionation of $7 \mathbf{a}$ under basic conditions has seldom been explored. ${ }^{12}$ This disproportionation was proposed to involve an initial isomerization process of 7a to give imine intermediate $\mathbf{8 a}$ (Scheme S8, eq. 1). Subsequent hydride transfer from anion species $\mathbf{1 4}$ to $\mathbf{8 a}$ could occur because the latter is a good electrophile (Scheme S8, eq. 2). The overall reaction of these two steps was a base-promoted disproportionation reaction of 7a (Scheme S8, eq. 3). This process was feasible with an activation energy of $23.4 \mathrm{kcal} / \mathrm{mol}$, as verified by DFT calculation (Figure S15). However, in the catalytic hydrogenation of quinoline (3a), the overall activation energy for disproportionation of 7a should be determined to be $27.3 \mathrm{kcal} / \mathrm{mol}$ considering the endotherm of $\mathbf{8 a}$, which is higher than the energy barrier of imine reduction process by $3.2 \mathrm{kcal} / \mathrm{mol}$ (Scheme 9 in text). Another competitive reaction pathway for the disproportionation of $\mathbf{7 a}$ without the assistance of quinoline was also calculated, with a higher energy barrier (Scheme S16). Therefore, the disproportionation is difficult to occur in catalytic hydrogenation of quinoline (3a). Furthermore, the isomerization of 7a could generate another anion intermediate (15). Intermediate $\mathbf{1 5}$ is a strong nucleophile that can react with electrophilic compounds, such as $\mathbf{3 a}$ and $\mathbf{8 a}$, to afford C 3 coupling products. ${ }^{13}$ Dimerization product 2,3'-biquinoline (16) and ring-opening product 17 were indeed isolated in $12 \%$ and $20 \%$ yields, respectively, from the reaction of $7 \mathbf{a}$ with $t \mathrm{BuOK}$. The formation of $\mathbf{1 6}$ and $\mathbf{1 7}$ strongly supported the existence of key anion intermediate $\mathbf{1 5}$, which initiated nucleophilic attack at the $\mathrm{C} 3$ position. Two possible pathways to generate intermediates $\mathbf{2 0}$ or $\mathbf{2 1}$ were proposed (Scheme S8, eqs. 4 and 5), which could be oxidized in air to form aromatic product 16 during the workup process. ${ }^{14}$ The nucleophilic attack of $\mathbf{3 a}$ by $\mathbf{1 5}$ had to overcome an energy barrier of 25.9 
$\mathrm{kcal} / \mathrm{mol}$ (Figure S15), that was higher than the overall activation free energy of the isomerization and subsequent imine reduction steps by $1.8 \mathrm{kcal} / \mathrm{mol}$ (Scheme 9 in text). Therefore, the dimerization cannot be observed in the catalytic hydrogenation reaction either. Nevertheless, the observed disproportionation and dimerization reactions in the absence of Mn catalyst provided strong evidence for the feasibility of the base-promoted isomerization of $\mathbf{7 a}$ to form imine intermediate $\mathbf{8 a}$.

2,3'-biquinoline 16 as white solid (31 mg, $0.12 \mathrm{mmol}, 12 \%):{ }^{15}{ }^{1} \mathrm{H} \mathrm{NMR}\left(400 \mathrm{MHz}, \mathrm{CDCl}_{3}\right) \delta 9.76(\mathrm{~d}, \mathrm{~J}$ $=2.2 \mathrm{~Hz}, 1 \mathrm{H}), 8.92(\mathrm{~d}, \mathrm{~J}=2.2 \mathrm{~Hz}, 1 \mathrm{H}), 8.31(\mathrm{~d}, \mathrm{~J}=8.6 \mathrm{~Hz}, 1 \mathrm{H}), 8.23(\mathrm{~d}, \mathrm{~J}=8.5 \mathrm{~Hz}, 1 \mathrm{H}), 8.19(\mathrm{~d}, \mathrm{~J}=$ $8.4 \mathrm{~Hz}, 1 \mathrm{H}), 8.03(\mathrm{~d}, \mathrm{~J}=8.6 \mathrm{~Hz}, 1 \mathrm{H}), 7.98(\mathrm{~d}, \mathrm{~J}=8.1 \mathrm{~Hz}, 1 \mathrm{H}), 7.87$ (d, J = 8.1 Hz, $1 \mathrm{H}), 7.78(\mathrm{td}, \mathrm{J}=$ 7.7, $3.9 \mathrm{~Hz}, 2 \mathrm{H}), 7.59$ (dt, J = 12.4, 7.6 Hz, $2 \mathrm{H}) .{ }^{13} \mathrm{C} \mathrm{NMR}\left(101 \mathrm{MHz}, \mathrm{CDCl}_{3}\right) \delta 154.7,149.8,148.5$, 148.4, 137.2, 134.5, 132.1, 130.1, 130.0, 129.8, 129.3, 128.6, 127.8, 127.6, 127.4, 127.0, 126.8, 118.7.

Single crystal of $\mathbf{1 6}$ suitable for X-ray diffraction measurements were crystallized from saturated ethyl acetate solution via evaporation (Figure S9).

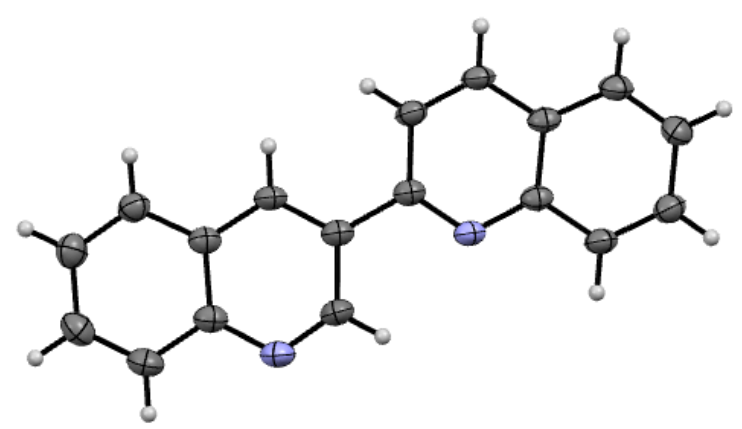

Figure S9. Single crystal of 2,3'-biquinoline $\mathbf{1 6}$

2-(3-(quinolin-3-yl)propyl)aniline 17 as pale yellow oil (52 mg, $0.2 \mathrm{mmol}, 20 \%)$ : ${ }^{1} \mathrm{H}$ NMR (400 MHz, $\left.\mathrm{CDCl}_{3}\right) \delta 8.81(\mathrm{~d}, \mathrm{~J}=2.2 \mathrm{~Hz}, 1 \mathrm{H}), 8.09(\mathrm{~d}, \mathrm{~J}=8.5 \mathrm{~Hz}, 1 \mathrm{H}), 7.94(\mathrm{~d}, \mathrm{~J}=2.1 \mathrm{~Hz}, 1 \mathrm{H}), 7.77(\mathrm{dd}, \mathrm{J}=8.3$, $1.3 \mathrm{~Hz}, 1 \mathrm{H}), 7.74-7.61(\mathrm{~m}, 1 \mathrm{H}), 7.53$ (d, J = 7.6 Hz, $1 \mathrm{H}), 7.06$ (d, J = 7.5 Hz, $2 \mathrm{H})$, 6.82-6.64 (m, $2 \mathrm{H})$, 3.57 (s, $2 \mathrm{H}), 2.90(\mathrm{t}, \mathrm{J}=7.7 \mathrm{~Hz}, 2 \mathrm{H}), 2.67-2.52(\mathrm{~m}, 2 \mathrm{H}), 2.09$ (p, J = 7.7 Hz, $2 \mathrm{H}) .{ }^{13} \mathrm{C} \mathrm{NMR}(101$ $\left.\mathrm{MHz}, \mathrm{CDCl}_{3}\right) \delta 151.9,146.8,144.0,134.6,134.1,129.4,129.01,128.6,128.1,127.2,127.1,126.5$, 125.6, 118.7, 115.6, 32.8, 30.6, 29.6.

HRMS (ESI) calcd. for $\mathrm{C}_{18} \mathrm{H}_{18} \mathrm{~N}_{2}[\mathrm{M}+\mathrm{H}]^{+}:$263.1543; found: 263.1534 .

2-(3-(quinolin-3-yl)propyl)aniline 17 (26 mg, $0.1 \mathrm{mmol}$ ) was dissolved in $3 \mathrm{~mL}$ of dichloromethane and $\mathrm{HCl} \cdot \mathrm{Et}_{2} \mathrm{O}$ (2 equiv.) solution was added. White solid was immediately produced and collected by filter. Single crystal of $\mathbf{1 7} \cdot 2 \mathrm{HCl} \cdot 2 \mathrm{H}_{2} \mathrm{O}$ suitable for X-ray diffraction measurements were crystallized from saturated methanol solution via evaporation (Figure S10).

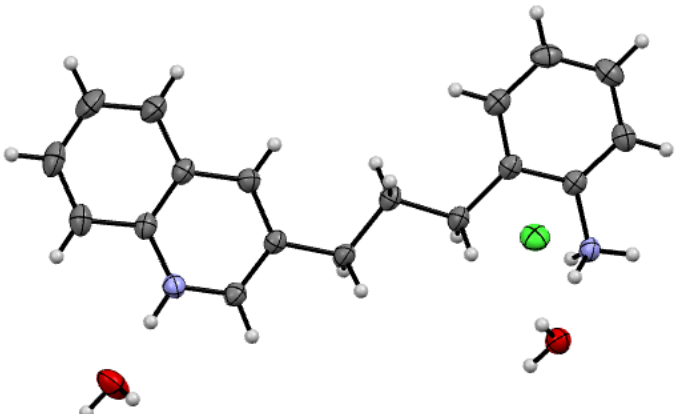


Figure S10. Single crystal of 2-(3-(quinolin-3-yl)propyl)aniline $17 \cdot 2 \mathrm{HCl} \cdot 2 \mathrm{H}_{2} \mathrm{O}$

\title{
Summary of Crystallographic data
}

\begin{tabular}{|c|c|c|c|c|}
\hline Compound & [Mn]-1a & {$[\mathrm{Mn}]-\mathbf{4 a}$} & 16 & $17 \cdot 2 \mathrm{HCl} \cdot 2 \mathrm{H}_{2} \mathrm{O}$ \\
\hline $\mathrm{CCDC}$ & 1919494 & 1919493 & 1919425 & 1919492 \\
\hline Formula & $\mathrm{C} 15 \mathrm{H} 25 \mathrm{MnN} 3 \mathrm{O} 2 \mathrm{P}$ & $\mathrm{C} 22 \mathrm{H} 20 \mathrm{MnN} 2 \mathrm{O} 2 \mathrm{P}$ & $\mathrm{C} 18 \mathrm{H} 12 \mathrm{~N} 2$ & $2(\mathrm{Cl}), 2(\mathrm{H} 2 \mathrm{O}), \mathrm{C} 18 \mathrm{H} 20 \mathrm{~N} 2$ \\
\hline Crystal system & monoclinic & triclinic & monoclinic & triclinic \\
\hline Space group & P $121 / \mathrm{c} 1$ & P -1 & P $121 / \mathrm{c} 1$ & $\mathrm{P}-1$ \\
\hline$a[\AA]]$ & $7.5799(2)$ & $8.2412(4)$ & $19.0886(11)$ & $7.5041(2)$ \\
\hline $\mathrm{b}[\AA]$ & $31.5365(6)$ & $9.6510(5)$ & $5.3694(3)$ & $8.7549(4)$ \\
\hline$c[\AA]$ & $7.9293(2)$ & $14.0685(7)$ & $12.4207(5)$ & $14.4335(7)$ \\
\hline$\alpha\left[^{\circ}\right]$ & 90 & $104.314(4)$ & 90 & $87.482(4)$ \\
\hline$\beta\left[^{\circ}\right]$ & $112.297(3)$ & $98.410(4)$ & $100.029(4)$ & $85.275(3)$ \\
\hline$\gamma\left[^{\circ}\right]$ & 90 & $107.805(4)$ & 90 & $87.737(3)$ \\
\hline Cell volume[Å3] & $1753.72(8)$ & 1001.99(9) & $1253.60(11)$ & $943.50(7)$ \\
\hline $\mathrm{Z}$ & 4 & 2 & 4 & 2 \\
\hline Crystal size $\left[\mathrm{mm}^{3}\right]$ & $0.3 \times 0.15 \times 0.02$ & $0.12 \times 0.08 \times 0.02$ & $0.4 \times 0.08 \times 0.08$ & $0.25 \times 0.15 \times 0.08$ \\
\hline Density $\left[\mathrm{Mg} / \mathrm{m}^{3}\right]$ & 1.384 & 1.426 & 1.358 & 1.307 \\
\hline $\mathrm{T}[\mathrm{K}]$ & $172.99(10)$ & $172.99(10)$ & $172.99(10)$ & $172.98(10)$ \\
\hline Theta range $\left[^{\circ}\right]$ & 5.611 to 71.306 & 5.063 to 76.478 & 4.705 to 75.906 & 5.060 to 74.779 \\
\hline Reflections collected & 6760 & 6819 & 4318 & 6168 \\
\hline
\end{tabular}

\section{Characterization Data of Quinoline Derivatives}

\author{
1,2,3,4-tetrahydroisoquinoline $4 \mathrm{a}^{2}$<smiles>c1ccc2c(c1)CCCN2</smiles> \\ 4a
}

Product was isolated via column chromatography $(\mathrm{PE} / \mathrm{EA}=10: 1)$ as yellow oil $(451 \mathrm{mg}, 2.33 \mathrm{mmol}$, 99\%). ${ }^{1} \mathrm{H}$ NMR (400 MHz, $\mathrm{CDCl}_{3}$ ) $\delta 7.11-6.95$ (m, $2 \mathrm{H}$ ), 6.70 (q, J=6.8 Hz, $1 \mathrm{H}$ ), 6.56 (d, J = 8.1 Hz, 1 H), 3.81 (s, $1 \mathrm{H}), 3.35$ (q, J = 4.2, $2.9 \mathrm{~Hz}, 2 \mathrm{H}), 2.83$ (q, J = 5.8, 5.2 Hz, $2 \mathrm{H}), 2.01$ (q, J = 5.6 Hz, $2 \mathrm{H}$ ). ${ }^{13} \mathrm{C}$ NMR $\left(101 \mathrm{MHz}, \mathrm{CDCl}_{3}\right) \delta 144.7,129.4,126.6,121.3,116.8,114.0,41.9,26.9,22.1$.

\section{6-methyl-1,2,3,4 tetrahydroquinoline $4 b^{2}$}<smiles>Cc1ccc2c(c1)CCCN2</smiles>

Product was isolated via column chromatography $(\mathrm{PE} / \mathrm{EA}=10: 1)$ as yellow oil $(34.9 \mathrm{mg}, 0.238 \mathrm{mmol}$, 95\%). ${ }^{1} \mathrm{H}$ NMR (400 MHz, $\left.\mathrm{CDCl}_{3}\right) \delta 6.81(\mathrm{~d}, \mathrm{~J}=4.3 \mathrm{~Hz}, 2 \mathrm{H}), 6.43(\mathrm{~d}, \mathrm{~J}=8.5 \mathrm{~Hz}, 1 \mathrm{H}), 3.66(\mathrm{~s}, 1 \mathrm{H})$, 3.37-3.19 (m, $2 \mathrm{H}), 2.76(\mathrm{t}, \mathrm{J}=6.5 \mathrm{~Hz}, 2 \mathrm{H}), 2.24(\mathrm{~s}, 3 \mathrm{H}), 2.01-1.87$ (m, $2 \mathrm{H}) .{ }^{13} \mathrm{C} \mathrm{NMR}(101 \mathrm{MHz}$, 


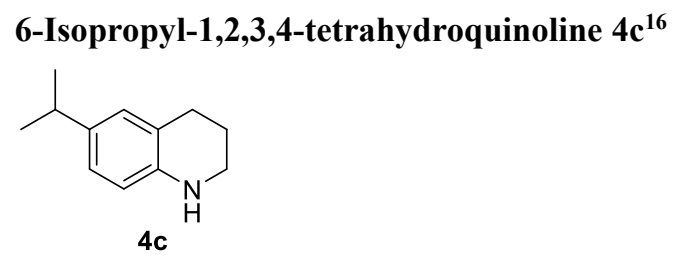

Product was isolated via column chromatography $(\mathrm{PE} / \mathrm{EA}=10: 1)$ as yellow oil $(39.8 \mathrm{mg}, 0.228 \mathrm{mmol}$, 91\%). ${ }^{1} \mathrm{H}$ NMR (400 MHz, $\mathrm{CDCl}_{3}$ ) $\delta 6.87$ (dd, J = 10.7, $\left.2.6 \mathrm{~Hz}, 2 \mathrm{H}\right), 6.47$ (d, J = 8.0 Hz, $1 \mathrm{H}$ ), 3.69 (s, $1 \mathrm{H}), 3.36-3.23$ (m, $2 \mathrm{H}), 2.79$ (dd, J = 8.1, $4.8 \mathrm{~Hz}, 3 \mathrm{H}), 1.97$ (p, J = 6.2 Hz, $2 \mathrm{H}), 1.24$ (d, J = 6.9 Hz, 6 H). ${ }^{13} \mathrm{C} \mathrm{NMR}\left(101 \mathrm{MHz}, \mathrm{CDCl}_{3}\right) \delta 142.7,137.6,127.4,124.6,121.3,114.4,42.1,33.2,27.0,24.3,22.4$.

\section{1,2,3,4-tetrahydroquinoline-6-carbonitrile $4 \mathrm{~d}^{17}$}<smiles>N#Cc1ccc2c(c1)CCCN2</smiles>

Product was isolated via column chromatography $(\mathrm{PE} / \mathrm{EA}=10: 1)$ as white solid $(25.7 \mathrm{mg}, 0.16 \mathrm{mmol}$, 65\%). ${ }^{1} \mathrm{H}$ NMR (400 MHz, $\left.\mathrm{CDCl}_{3}\right) \delta 7.20(\mathrm{~d}, \mathrm{~J}=10.9 \mathrm{~Hz}, 2 \mathrm{H}), 6.38(\mathrm{~d}, \mathrm{~J}=8.3 \mathrm{~Hz}, 1 \mathrm{H}), 4.36(\mathrm{~s}, 1 \mathrm{H})$, $3.36(\mathrm{t}, \mathrm{J}=5.6 \mathrm{~Hz}, 2 \mathrm{H}), 2.73(\mathrm{t}, \mathrm{J}=6.4 \mathrm{~Hz}, 2 \mathrm{H}), 1.92(\mathrm{p}, \mathrm{J}=6.1 \mathrm{~Hz}, 2 \mathrm{H}) .{ }^{13} \mathrm{C} \mathrm{NMR}\left(101 \mathrm{MHz}, \mathrm{CDCl}_{3}\right)$ $\delta 148.2,133.2,131.3,120.9,120.8,113.2,97.8,41.6,26.7,21.0$.

methyl 1,2,3,4-tetrahydroquinoline-6-carboxylate $4 \mathrm{e}^{2}$<smiles>CC(=O)c1ccc2c(c1)CCCN2</smiles>

Product was isolated via column chromatography $(\mathrm{PE} / \mathrm{EA}=10: 1)$ as white solid (44.9 mg, $0.235 \mathrm{mmol}$, 94\%). ${ }^{1} \mathrm{H}$ NMR (400 MHz, $\mathrm{CDCl}_{3}$ ) $\delta$ 7.69-7.59 (m, $\left.2 \mathrm{H}\right), 6.39$ (d, J = 8.9 Hz, $1 \mathrm{H}$ ), 4.33 (s, $\left.1 \mathrm{H}\right), 3.83$ (s, $3 \mathrm{H}), 3.35(\mathrm{t}, \mathrm{J}=5.6 \mathrm{~Hz}, 2 \mathrm{H}), 2.76(\mathrm{t}, \mathrm{J}=6.3 \mathrm{~Hz}, 2 \mathrm{H}), 1.99-1.87(\mathrm{~m}, 2 \mathrm{H}) .{ }^{13} \mathrm{C} \mathrm{NMR}\left(101 \mathrm{MHz}, \mathrm{CDCl}_{3}\right)$ $\delta 167.5,148.78131 .3,129.1,119.9,117.4,112.6,51.4,41.7,26.9,21.4$.

\section{6-methoxy-1,2,3,4-tetrahydroquinoline $4 f^{2}$}<smiles>COc1ccc2c(c1)CCCN2</smiles>

Product was isolated via column chromatography $(\mathrm{PE} / \mathrm{EA}=10: 1)$ as yellow oil $(34.6 \mathrm{mg}, 0.212 \mathrm{mmol}$, 85\%). ${ }^{1} \mathrm{H}$ NMR (400 MHz, $\left.\mathrm{CDCl}_{3}\right) \delta$ 6.65-6.53 (m, $\left.2 \mathrm{H}\right), 6.46(\mathrm{~d}, \mathrm{~J}=8.5 \mathrm{~Hz}, 1 \mathrm{H}), 3.73(\mathrm{~s}, 3 \mathrm{H}), 3.50(\mathrm{~s}$, $1 \mathrm{H}), 3.31-3.21(\mathrm{~m}, 2 \mathrm{H}), 2.76(\mathrm{t}, \mathrm{J}=6.5 \mathrm{~Hz}, 2 \mathrm{H}), 2.00-1.86(\mathrm{~m}, 2 \mathrm{H}) .{ }^{13} \mathrm{C} \mathrm{NMR}\left(101 \mathrm{MHz}, \mathrm{CDCl}_{3}\right) \delta$ $151.8,138.8,122.9,115.5,114.9,112.9,55.8,42.3,27.1,22.4$.

6-(methylthio)-1,2,3,4-tetrahydroquinoline $4 \mathrm{~g}^{18}$ 
<smiles>Cc1ccc2c(c1)CCCN2</smiles>

Product was isolated via column chromatography ( $\mathrm{PE} / \mathrm{EA}=10: 1)$ as purple solid $(34.0 \mathrm{mg}, 0.190 \mathrm{mmol}$, 76\%). ${ }^{1} \mathrm{H}$ NMR (400 MHz, $\mathrm{CDCl}_{3}$ ) $\delta 7.02(\mathrm{dt}, \mathrm{J}=3.7,1.8 \mathrm{~Hz}, 2 \mathrm{H}), 6.42(\mathrm{~d}, \mathrm{~J}=8.8 \mathrm{~Hz}, 1 \mathrm{H}), 3.83$ (s, 1 $\mathrm{H}), 3.29(\mathrm{t}, \mathrm{J}=5.5 \mathrm{~Hz}, 2 \mathrm{H}), 2.74(\mathrm{t}, \mathrm{J}=6.5 \mathrm{~Hz}, 2 \mathrm{H}), 2.41(\mathrm{~s}, 3 \mathrm{H}), 2.01-1.84(\mathrm{~m}, 2 \mathrm{H}) .{ }^{13} \mathrm{C}$ NMR $(101$ $\left.\mathrm{MHz}, \mathrm{CDCl}_{3}\right) \delta 143.7,131.1,129.1,123.3,122.0,114.6,41.8,26.8,21.9,19.3$.

\section{6-fluoro-1,2,3,4-tetrahydroquinoline $4 \mathrm{~h}^{19}$}<smiles>Fc1ccc2c(c1)CCC2</smiles>

Product was isolated via column chromatography $(\mathrm{PE} / \mathrm{EA}=10: 1)$ as yellow oil $(32.4 \mathrm{mg}, 0.215 \mathrm{mmol}$, 86\%). ${ }^{1} \mathrm{H}$ NMR (400 MHz, $\left.\mathrm{CDCl}_{3}\right) \delta$ 6.77-6.59 (m, 2H), 6.40 (dd, J = 9.5, $\left.4.8 \mathrm{~Hz}, 1 \mathrm{H}\right), 3.70(\mathrm{~s}, 1 \mathrm{H})$, 3.35-3.19 (m, $2 \mathrm{H}), 2.75$ (t, J = 6.5 Hz, $2 \mathrm{H}), 2.04-1.80$ (m, $2 \mathrm{H}) .{ }^{13} \mathrm{C}$ NMR $\left(101 \mathrm{MHz}, \mathrm{CDCl}_{3}\right) \delta 155.4$ (d, $J=234.5 \mathrm{~Hz}), 140.9,122.7$ (d, $J=6.8 \mathrm{~Hz}), 115.6$ (d, $J=21.6 \mathrm{~Hz}), 114.8(\mathrm{~d}, J=7.6 \mathrm{~Hz}), 113.1(\mathrm{~d}, J$ $=22.4 \mathrm{~Hz}), 42.0,27.0,22.0$.

\section{6-chloro-1,2,3,4-tetrahydroquinoline $4 \mathrm{i}^{2}$}<smiles>Clc1ccc2c(c1)CCCN2</smiles>

Product was isolated via column chromatography $(\mathrm{PE} / \mathrm{EA}=10: 1)$ as yellow oil $(35.1 \mathrm{mg}, 0.21 \mathrm{mmol}$, 84\%). ${ }^{1} \mathrm{H}$ NMR (400 MHz, CDCl $) \delta 6.90(\mathrm{~d}, \mathrm{~J}=8.0 \mathrm{~Hz}, 2 \mathrm{H}), 6.38(\mathrm{~d}, \mathrm{~J}=8.2 \mathrm{~Hz}, 1 \mathrm{H}), 3.81(\mathrm{~s}, 1 \mathrm{H})$, 3.37-3.19 (m, $2 \mathrm{H}), 2.73(\mathrm{t}, \mathrm{J}=6.4 \mathrm{~Hz}, 2 \mathrm{H}), 1.91$ (dt, J = 11.3, $6.3 \mathrm{~Hz}, 2 \mathrm{H}) .{ }^{13} \mathrm{C}$ NMR $\left(101 \mathrm{MHz}, \mathrm{CDCl}_{3}\right)$ $\delta 143.3,129.0,126.5,122.8,121.2,115.0,41.8,26.9,21.7$.

\section{6-bromo-1,2,3,4-tetrahydroquinoline $4 \mathrm{j}^{2}$}<smiles>Brc1ccc2c(c1)CCCN2</smiles>

Product was isolated via column chromatography $(\mathrm{PE} / \mathrm{EA}=10: 1)$ as yellow oil $(40.8 \mathrm{mg}, 0.19 \mathrm{mmol}$, 77\%). ${ }^{1} \mathrm{H}$ NMR (400 MHz, $\left.\mathrm{CDCl}_{3}\right) \delta$ 7.11-6.96 (m, $\left.2 \mathrm{H}\right), 6.34(\mathrm{~d}, \mathrm{~J}=8.3 \mathrm{~Hz}, 1 \mathrm{H}), 3.77(\mathrm{~s}, 1 \mathrm{H}), 3.28$ (t, $\mathrm{J}=5.4 \mathrm{~Hz}, 2 \mathrm{H}), 2.73(\mathrm{t}, \mathrm{J}=6.4 \mathrm{~Hz}, 2 \mathrm{H}), 1.92(\mathrm{q}, \mathrm{J}=5.9 \mathrm{~Hz}, 2 \mathrm{H}) .{ }^{13} \mathrm{C} \mathrm{NMR}\left(101 \mathrm{MHz}, \mathrm{CDCl}_{3}\right) \delta$ 143.6, $131.8,129.3,123.4,115.5,108.2,41.7,26.8,21.6$.

\section{5-bromo-1,2,3,4-tetrahydroquinoline $4 \mathrm{k}^{2}$}


<smiles>Brc1cccc2c1CCCN2</smiles>

Product was isolated via column chromatography $(\mathrm{PE} / \mathrm{EA}=10: 1)$ as yellow oil $(39.8 \mathrm{mg}, 0.188 \mathrm{mmol}$, 75\%). ${ }^{1} \mathrm{H}$ NMR (400 MHz, $\left.\mathrm{CDCl}_{3}\right) \delta$ 6.94-6.74 (m, $\left.2 \mathrm{H}\right), 6.41$ (dd, J = 7.9, $\left.1.3 \mathrm{~Hz}, 1 \mathrm{H}\right), 3.92(\mathrm{~s}, 1$ $\mathrm{H}), 3.34-3.18(\mathrm{~m}, 2 \mathrm{H}), 2.78(\mathrm{t}, \mathrm{J}=6.5 \mathrm{~Hz}, 2 \mathrm{H}), 2.04-1.90(\mathrm{~m}, 2 \mathrm{H}) .{ }^{13} \mathrm{C} \mathrm{NMR}\left(101 \mathrm{MHz}, \mathrm{CDCl}_{3}\right) \delta$ $146.4,127.5,125.9,120.7,120.6,113.1,41.4,27.6,22.2$.

\section{8-methyl-1,2,3,4-tetrahydroquinoline $4 \mathbf{l}^{20}$}<smiles>Cc1cccc2c1NCCC2</smiles>

Product was isolated via column chromatography $\left(\mathrm{CH}_{2} \mathrm{Cl}_{2} / \mathrm{MeOH}=1: 1\right)$ as yellow oil (35.6 mg, 0.243 mmol, 97\%). ${ }^{1} \mathrm{H}$ NMR (400 MHz, $\left.\mathrm{CDCl}_{3}\right) \delta 6.91(\mathrm{dd}, J=11.1,7.8 \mathrm{~Hz}, 2 \mathrm{H}), 6.61$ (ddd, $J=7.5,4.6$, $2.9 \mathrm{~Hz}, 1 \mathrm{H}), 3.68$ (s, $1 \mathrm{H}), 3.47-3.37$ (m, $2 \mathrm{H}), 2.84$ (td, $J=6.6,2.5 \mathrm{~Hz}, 2 \mathrm{H}), 2.13$ (s, $3 \mathrm{H}), 2.02-$ $1.96(\mathrm{~m}, 2 \mathrm{H}) .{ }^{13} \mathrm{C} \mathrm{NMR}\left(101 \mathrm{MHz}, \mathrm{CDCl}_{3}\right) \delta 142.6,127.8,127.3,121.1,120.8,116.3,42.3,27.3,22.1$, 17.1.

\section{6-(phenylethynyl)-1,2,3,4-tetrahydroquinoline $4 \mathrm{~m}^{2}$}<smiles>C(#Cc1ccc2c(c1)CCCN2)c1ccccc1</smiles>

Product was isolated via column chromatography $(\mathrm{PE} / \mathrm{EA}=10: 1)$ as white solid $(43.7 \mathrm{mg}, 0.188 \mathrm{mmol}$, 75\%). ${ }^{1} \mathrm{H}$ NMR (400 MHz, $\mathrm{CDCl}_{3}$ ) $\delta 7.56(\mathrm{dd}, \mathrm{J}=7.6,2.6 \mathrm{~Hz}, 2 \mathrm{H}), 7.37$ (dt, J = 11.8, 7.0 Hz, $\left.3 \mathrm{H}\right), 7.22$ $(\mathrm{d}, \mathrm{J}=4.4 \mathrm{~Hz}, 2 \mathrm{H}), 6.43(\mathrm{~d}, \mathrm{~J}=8.7 \mathrm{~Hz}, 1 \mathrm{H}), 4.02(\mathrm{~s}, 1 \mathrm{H}), 3.34(\mathrm{t}, \mathrm{J}=5.5 \mathrm{~Hz}, 2 \mathrm{H}), 2.79(\mathrm{t}, \mathrm{J}=6.4 \mathrm{~Hz}$, $2 \mathrm{H}), 1.97$ (p, J = 6.0 Hz, $2 \mathrm{H}) .{ }^{13} \mathrm{C} \mathrm{NMR}\left(101 \mathrm{MHz}, \mathrm{CDCl}_{3}\right) \delta 144.9,132.7,131.1,130.4,128.1,127.3$, $124.1,120.8,113.5,110.2,90.8,86.8,41.7,26.7,21.7$.

\section{6-styryl-1,2,3,4-tetrahydroquinoline $4 n^{2}$}<smiles>C(=C/c1ccc2c(c1)CCCN2)\c1ccccc1</smiles>

Product was isolated via column chromatography $(\mathrm{PE} / \mathrm{EA}=10: 1)$ as white solid (48.8 $\mathrm{mg}, 0.208 \mathrm{mmol}$, 83\%). ${ }^{1} \mathrm{H}$ NMR (400 MHz, $\mathrm{CDCl}_{3}$ ) $\delta 7.47$ (d, J = 7.7 Hz, $2 \mathrm{H}$ ), 7.33 (t, J = 7.6 Hz, $\left.2 \mathrm{H}\right), 7.24-7.13$ (m, 3 H), $7.01(\mathrm{~d}, \mathrm{~J}=16.3 \mathrm{~Hz}, 1 \mathrm{H}), 6.89(\mathrm{~d}, \mathrm{~J}=16.2 \mathrm{~Hz}, 1 \mathrm{H}), 6.47(\mathrm{~d}, \mathrm{~J}=8.0 \mathrm{~Hz}, 1 \mathrm{H}), 4.09(\mathrm{~s}, 1 \mathrm{H}), 3.34$ (t, $\mathrm{J}=5.5 \mathrm{~Hz}, 2 \mathrm{H}), 2.80(\mathrm{t}, \mathrm{J}=6.4 \mathrm{~Hz}, 2 \mathrm{H}), 1.96(\mathrm{p}, \mathrm{J}=6.2 \mathrm{~Hz}, 2 \mathrm{H}) .{ }^{13} \mathrm{C} \mathrm{NMR}\left(101 \mathrm{MHz}, \mathrm{CDCl}_{3}\right) \delta 144.5$, 138.2, 129.0, 128.5, 127.8, $126.60126 .4,125.9,125.4,124.0,121.3,114.2,42.0,27.0,22.1$. 


\section{1,2,3,4-tetrahydro-2-methylquinoline $40^{2}$}<smiles>CC1CCc2ccccc2N1</smiles>

Product was isolated via column chromatography $(\mathrm{PE} / \mathrm{EA}=10: 1)$ as yellow oil $(32.0 \mathrm{mg}, 0.218 \mathrm{mmol}$, 87\%). ${ }^{1} \mathrm{H}$ NMR (400 MHz, $\left.\mathrm{CDCl}_{3}\right) \delta$ 7.05-6.94 (m, $\left.2 \mathrm{H}\right), 6.63(\mathrm{td}, \mathrm{J}=7.5,3.0 \mathrm{~Hz}, 1 \mathrm{H}), 6.54-6.45(\mathrm{~m}, 1$ H), $3.70(\mathrm{~s}, 1 \mathrm{H}), 3.43$ (ddp, J = 9.7, 6.6, 3.4 Hz, $1 \mathrm{H}), 2.95-2.81(\mathrm{~m}, 1 \mathrm{H}), 2.82-2.68$ (m, $1 \mathrm{H}), 1.96$ (ddd, $\mathrm{J}=12.6,5.9,3.1 \mathrm{~Hz}, 1 \mathrm{H}), 1.74-1.52(\mathrm{~m}, 1 \mathrm{H}), 1.30-1.16(\mathrm{~m}, 3 \mathrm{H}) .{ }^{13} \mathrm{C} \mathrm{NMR}\left(101 \mathrm{MHz}, \mathrm{CDCl}_{3}\right) \delta 144.7$, $129.2,126.6,121.0,116.9,114.0,47.1,30.1,26.6,22.6$.

2-phenyl-1,2,3,4-tetrahydroquinoline $4 p^{2}$<smiles></smiles>

Product was isolated via column chromatography $(\mathrm{PE} / \mathrm{EA}=10: 1)$ as yellow oil $(46.0 \mathrm{mg}, 0.220 \mathrm{mmol}$, 88\%). ${ }^{1} \mathrm{H}$ NMR (400 MHz, $\mathrm{CDCl}_{3}$ ) $\delta$ 7.49-7.38 (m, $\left.4 \mathrm{H}\right), 7.35$ (dd, J = 8.3, 5.2 Hz, $\left.1 \mathrm{H}\right), 7.07$ (q, J = 5.7, $3.3 \mathrm{~Hz}, 2 \mathrm{H}), 6.76-6.67$ (m, $1 \mathrm{H}), 6.59$ (d, J = 7.9 Hz, $1 \mathrm{H}), 4.49$ (dd, J = 9.4, 3.1 Hz, $1 \mathrm{H}), 4.07$ (s, $1 \mathrm{H})$, 2.98 (ddd, J = 16.3, 10.6, 5.4 Hz, $1 \mathrm{H}), 2.80$ (dt, J = 16.4, 4.6 Hz, $1 \mathrm{H}), 2.18$ (dq, J = 13.3, 4.4 Hz, $1 \mathrm{H}$ ), 2.05 (tdd, $\mathrm{J}=13.3,8.8,3.7 \mathrm{~Hz}, 1 \mathrm{H}) .{ }^{13} \mathrm{C} \mathrm{NMR}\left(101 \mathrm{MHz}, \mathrm{CDCl}_{3}\right) \delta 144.8,144.7,129.2,128.5,127.4$, $126.8,126.5,120.8,117.1,113.9,56.2,30.9,26.3$.

\section{1,2,3,4-tetrahydro-2,2'-biquinoline $4 q^{21}$}<smiles>c1ccc2c(c1)CCC(c1ccc3ccccc3n1)N2</smiles>

$4 q$

Product was isolated via column chromatography $(\mathrm{PE} / \mathrm{EA}=10: 1)$ as white solid $(33.2 \mathrm{mg}, 0.128 \mathrm{mmol}$, 51\%). ${ }^{1} \mathrm{H}$ NMR (400 MHz, $\left.\mathrm{CDCl}_{3}\right) \delta 8.13(\mathrm{t}, \mathrm{J}=8.9 \mathrm{~Hz}, 2 \mathrm{H}), 7.82(\mathrm{~d}, \mathrm{~J}=8.1 \mathrm{~Hz}, 1 \mathrm{H}), 7.74(\mathrm{t}, \mathrm{J}=7.7$ $\mathrm{Hz}, 1 \mathrm{H}), 7.55$ (dd, J = 8.2, 2.4 Hz, 2 H), 7.14-7.00 (m, 2 H), 6.71 (d, J = 7.5 Hz, $2 \mathrm{H}), 4.86$ (s, $1 \mathrm{H}), 4.77$ $(\mathrm{dd}, \mathrm{J}=9.6,3.4 \mathrm{~Hz}, 1 \mathrm{H}), 3.05-2.97$ (m, $1 \mathrm{H}), 2.82-2.76$ (m, $1 \mathrm{H}), 2.40-2.33$ (m, $1 \mathrm{H}), 2.15-1.97$ (m, 1 H). ${ }^{13} \mathrm{C}$ NMR $\left(101 \mathrm{MHz}, \mathrm{CDCl}_{3}\right) \delta 162.9,147.4,144.1,136.7,129.5,129.2,129.0,127.5,127.4,126.9$, $126.2,121.0,118.8,117.2,114.5,57.5,29.0,26.5$.

\section{4-methyl-1,2,3,4-tetrahydroquinoline $4 r^{2}$}<smiles>[C-]C1CCNc2ccccc21</smiles>

Product was isolated via column chromatography $(\mathrm{PE} / \mathrm{EA}=10: 1)$ as yellow oil $(25.0 \mathrm{mg}, 0.17 \mathrm{mmol}$, 68\%). ${ }^{1} \mathrm{H}$ NMR (400 MHz, $\left.\mathrm{CDCl}_{3}\right) \delta 7.07$ (d, J = 7.6 Hz, $\left.1 \mathrm{H}\right), 7.03-6.92$ (m, $\left.1 \mathrm{H}\right), 6.64$ (td, J = 7.4, 1.3 Hz, 1 H), 6.54-6.43 (m, 1 H), 3.85 (s, 1 H), 3.41-3.21 (m, 2 H), 2.93 (q, J = 6.5 Hz, 1 H), 2.00 (ddt, J = 
13.2, 8.9, 4.6 Hz, $1 \mathrm{H}), 1.69(\mathrm{dtd}, \mathrm{J}=12.9,6.4,3.6 \mathrm{~Hz}, 1 \mathrm{H}), 1.31(\mathrm{~d}, \mathrm{~J}=7.0 \mathrm{~Hz}, 3 \mathrm{H}) .{ }^{13} \mathrm{C}$ NMR $(101$ $\left.\mathrm{MHz}, \mathrm{CDCl}_{3}\right) \delta 144.2,128.4,126.7,126.6,116.9,114.1,39.0,30.2,29.9,22.6$.

4-isopropyl-1,2,3,4-tetrahydro-quinoline $4 \mathrm{~s}^{22}$<smiles>CC(C)C1CCNc2ccccc21</smiles>

Product was isolated via column chromatography $(\mathrm{PE} / \mathrm{EA}=10: 1)$ as yellow oil $(9.6 \mathrm{mg}, 0.055 \mathrm{mmol}$, 22\%). ${ }^{1} \mathrm{H}$ NMR (400 MHz, $\left.\mathrm{CDCl}_{3}\right) \delta$ 7.08-6.88 (m, $\left.2 \mathrm{H}\right), 6.61$ (t, J = 8.1 Hz, $\left.1 \mathrm{H}\right), 6.47$ (d, J = 9.1 Hz, 1 H), $3.81(\mathrm{~s}, 1 \mathrm{H}), 3.45-3.14(\mathrm{~m}, 2 \mathrm{H}), 2.55(\mathrm{q}, \mathrm{J}=6.0 \mathrm{~Hz}, 1 \mathrm{H}), 2.08-2.00(\mathrm{~m}, 1 \mathrm{H}), 1.97-1.90(\mathrm{~m}, 1 \mathrm{H})$, 1.85-1.77 (m, $1 \mathrm{H}), 1.00(\mathrm{~d}, \mathrm{~J}=6.8 \mathrm{~Hz}, 3 \mathrm{H}), 0.89(\mathrm{~d}, \mathrm{~J}=6.7 \mathrm{~Hz}, 3 \mathrm{H}) .{ }^{13} \mathrm{C} \mathrm{NMR}\left(101 \mathrm{MHz}, \mathrm{CDCl}_{3}\right) \delta$ 144.6, 129.3, 126.7, 124.4, 116.2, 114.0, 42.1, 39.5, 30.4, 22.9, 21.4, 18.4 .

2-phenethyl-1,2,3,4-tetrahydroquinoline $4 \mathrm{t}^{23}$<smiles>c1ccc(CCC2CCc3ccccc3N2)cc1</smiles>

$4 t$

Product was isolated via column chromatography $(\mathrm{PE} / \mathrm{EA}=10: 1)$ as yellow oil $(49.2 \mathrm{mg}, 0.208 \mathrm{mmol}$, 83\%). ${ }^{1} \mathrm{H}$ NMR (400 MHz, $\mathrm{CDCl}_{3}$ ) $\delta 7.37$ (t, J = $7.5 \mathrm{~Hz}, 2 \mathrm{H}$ ), 7.29 (d, J = $\left.7.4 \mathrm{~Hz}, 3 \mathrm{H}\right), 7.04$ (t, J = 7.6 Hz, 2 H), $6.68(\mathrm{t}, \mathrm{J}=7.4 \mathrm{~Hz}, 1 \mathrm{H}), 6.52(\mathrm{~d}, \mathrm{~J}=7.9 \mathrm{~Hz}, 1 \mathrm{H}), 3.84(\mathrm{~s}, 1 \mathrm{H}), 3.36(\mathrm{dp}, \mathrm{J}=9.3,3.1 \mathrm{~Hz}, 1 \mathrm{H})$, 2.96-2.72 (m, 4 H), 2.07 (dtd, J = 12.9, 5.1, 3.1 Hz, 1 H), 1.99-1.85 (m, 2 H), 1.75 (dtd, J = 12.7, 10.0, $5.6 \mathrm{~Hz}, 1 \mathrm{H}) .{ }^{13} \mathrm{C}$ NMR $\left(101 \mathrm{MHz}, \mathrm{CDCl}_{3}\right) \delta 144.4,141.8,129.2,128.4,128.3,126.7,125.9,121.2$, 117.0, 114.1, 51.1, 38.2, 32.1, 27.9, 26.2 .

8-chloro-2-methyl-1,2,3,4-tetrahydroquinoline $4 u^{2}$<smiles>CC1CCc2cccc(Cl)c2N1</smiles>

Product was isolated via column chromatography $(\mathrm{PE} / \mathrm{EA}=10: 1)$ as yellow oil $(30.3 \mathrm{mg}, 0.168 \mathrm{mmol}$, 67\%). ${ }^{1} \mathrm{H}$ NMR (400 MHz, $\mathrm{CDCl}_{3}$ ) $\delta 7.07$ (d, J = 8.0 Hz, 1 H), 6.87 (d, J = 7.4 Hz, 1H ), 6.51 (t, J = 7.7 Hz, $1 \mathrm{H}$ ), 4.27 (s, $1 \mathrm{H}$ ), 3.47 (ddd, J = 9.7, 6.3, 3.1 Hz, $1 \mathrm{H}$ ), 2.80 (dddd, J = 21.0, 16.4, 12.8, $5.1 \mathrm{~Hz}, 2$ $\mathrm{H}), 1.95(\mathrm{dq}, \mathrm{J}=12.8,4.1 \mathrm{~Hz}, 1 \mathrm{H}), 1.72-1.46(\mathrm{~m}, 1 \mathrm{H}), 1.28(\mathrm{~d}, \mathrm{~J}=6.3 \mathrm{~Hz}, 3 \mathrm{H}) .{ }^{13} \mathrm{C}$ NMR $(101 \mathrm{MHz}$, $\left.\mathrm{CDCl}_{3}\right) \delta 140.7,127.4,126.7,122.4,117.8,116.3,47.2,29.6,26.7,22.5$.

\section{Characterization Data of $N$-Heterocycles}

\section{1,2,3,4-tetrahydroisoquinoline $6 \mathrm{a}^{2}$}<smiles>c1ccc2c(c1)CCNC2</smiles>

$6 a$ 
Product was isolated via column chromatography $(\mathrm{PE} / \mathrm{EA}=10: 1)$ as yellow oil $(31.3 \mathrm{mg}, 0.235 \mathrm{mmol}$, 94\%). ${ }^{1} \mathrm{H}$ NMR (400 MHz, $\mathrm{CDCl}_{3}$ ) $\delta$ 7.18-7.07 (m, $\left.3 \mathrm{H}\right), 7.05-6.95$ (m, $\left.1 \mathrm{H}\right), 4.02$ (s, $\left.2 \mathrm{H}\right), 3.14$ (t, J = $6.0 \mathrm{~Hz}, 2 \mathrm{H}), 2.80(\mathrm{t}, \mathrm{J}=6.0 \mathrm{~Hz}, 2 \mathrm{H}), 1.72(\mathrm{~s}, 1 \mathrm{H}) .{ }^{13} \mathrm{C} \mathrm{NMR}\left(101 \mathrm{MHz}, \mathrm{CDCl}_{3}\right) \delta 136.0,134.8,129.3$, $126.2,126.0,125.7,48.4,43.9,29.2$.

\section{1,2,3,4-tetrahydroquinoxaline $6 b^{2}$}<smiles>CCNc1ccccc1NS(=O)(=O)O</smiles>

Product was isolated via column chromatography $(\mathrm{PE} / \mathrm{EA}=10: 1)$ as yellow oil $(30.2 \mathrm{mg}, 0.225 \mathrm{mmol}$, 90\%). ${ }^{1} \mathrm{H}$ NMR (400 MHz, $\left.\mathrm{CDCl}_{3}\right) \delta 6.60(\mathrm{dd}, \mathrm{J}=5.8,3.4 \mathrm{~Hz}, 2 \mathrm{H}), 6.50(\mathrm{dd}, \mathrm{J}=5.7,3.4 \mathrm{~Hz}, 2 \mathrm{H}), 3.62$ (s, $2 \mathrm{H}), 3.42$ (s, $4 \mathrm{H}) .{ }^{13} \mathrm{C} \mathrm{NMR}\left(101 \mathrm{MHz}, \mathrm{CDCl}_{3}\right) \delta 133.6,118.7,114.6,41.4$.

\section{1,2,3,4-tetrahydro-1,5-naphthyridine $6 c^{2}$}<smiles>O=C(Nc1cccnc1)c1ccccc1</smiles>

Product was isolated via column chromatography $(\mathrm{PE} / \mathrm{EA}=10: 1)$ as yellow oil $(29.5 \mathrm{mg}, 0.22 \mathrm{mmol}$, 88\%). ${ }^{1} \mathrm{H}$ NMR (400 MHz, $\left.\mathrm{CDCl}_{3}\right) \delta 7.85$ (d, J = 4.6 Hz, $\left.1 \mathrm{H}\right), 6.87(\mathrm{dd}, \mathrm{J}=8.1,4.4 \mathrm{~Hz}, 1 \mathrm{H}), 6.71(\mathrm{~d}, \mathrm{~J}$ $=7.9 \mathrm{~Hz}, 1 \mathrm{H}), 3.83(\mathrm{~s}, 1 \mathrm{H}), 3.29(\mathrm{t}, \mathrm{J}=5.4 \mathrm{~Hz}, 2 \mathrm{H}), 2.92(\mathrm{t}, \mathrm{J}=6.5 \mathrm{~Hz}, 2 \mathrm{H}), 2.02(\mathrm{p}, \mathrm{J}=5.9,5.4 \mathrm{~Hz}$, $2 \mathrm{H}) .{ }^{13} \mathrm{C} \mathrm{NMR}\left(101 \mathrm{MHz}, \mathrm{CDCl}_{3}\right) \delta 142.7,140.9,137.9,121.8,120.1,41.5,30.3,21.7$.

\section{1,2,3,4-tetrahydro-1,8-naphthyridine $6 d^{24}$}<smiles>c1cnc2c(c1)CCCN2</smiles>

Product was isolated via column chromatography $(\mathrm{PE} / \mathrm{EA}=10: 1)$ as yellow oil $(29.1 \mathrm{mg}, 0.218 \mathrm{mmol}$, 87\%). ${ }^{1} \mathrm{H}$ NMR (400 MHz, $\left.\mathrm{CDCl}_{3}\right) \delta 7.84(\mathrm{~d}, \mathrm{~J}=5.1 \mathrm{~Hz}, 1 \mathrm{H}), 7.12$ (d, J = 7.2 Hz, $\left.1 \mathrm{H}\right), 6.47(\mathrm{dd}, \mathrm{J}=$ 7.1, 4.6 Hz, 1 H), 5.01 (s, $1 \mathrm{H}), 3.40$ (t, J = 5.6 Hz, 2 H), 2.71 (t, J = 6.3 Hz, $2 \mathrm{H}), 2.00-1.82$ (m, $2 \mathrm{H})$. ${ }^{13} \mathrm{C} \mathrm{NMR}\left(101 \mathrm{MHz}, \mathrm{CDCl}_{3}\right) \delta 156.2,145.7,136.2,116.2,112.6,41.5,26.6,121.2$.

\section{1,2,3,4-tetrahydro-1,10-phenanthroline $6 \mathrm{e}^{20}$}<smiles>c1cnc2c3c(ccc2c1)CCCN3</smiles>

$6 e$

Product was isolated via column chromatography $(\mathrm{PE} / \mathrm{EA}=10: 1)$ as yellow oil $(42.3 \mathrm{mg}, 0.23 \mathrm{mmol}$, 92\%). ${ }^{1} \mathrm{H}$ NMR (400 MHz, $\left.\mathrm{CDCl}_{3}\right) \delta 8.69$ (dd, J = 4.3, $\left.1.6 \mathrm{~Hz}, 1 \mathrm{H}\right), 8.00(\mathrm{dd}, \mathrm{J}=8.2,1.7 \mathrm{~Hz}, 1 \mathrm{H}), 7.28$ $(\mathrm{dt}, \mathrm{J}=9.2,4.6 \mathrm{~Hz}, 1 \mathrm{H}), 7.16(\mathrm{~d}, \mathrm{~J}=8.2 \mathrm{~Hz}, 1 \mathrm{H}), 6.98(\mathrm{~d}, \mathrm{~J}=8.2 \mathrm{~Hz}, 1 \mathrm{H}), 5.94(\mathrm{~s}, 1 \mathrm{H}), 3.63-3.46$ (m, $2 \mathrm{H}), 2.93$ (t, J = 6.4 Hz, $2 \mathrm{H}), 2.08$ (p, J = 6.2 Hz, $2 \mathrm{H}) .{ }^{13} \mathrm{C} \mathrm{NMR}\left(101 \mathrm{MHz}, \mathrm{CDCl}_{3}\right) \delta 146.9,140.7$, $137.5,135.8,129.0,127.3,120.5,116.5,113.0,41.2,27.0,21.8$. 


\section{2,9-dimethyl-1,2,3,4-tetrahydro-1,10-phenanthroline $6 f^{25}$}<smiles>Cc1ccc2ccc3c(c2n1)NC(C)CC3</smiles>

Product was isolated via column chromatography $(\mathrm{PE} / \mathrm{EA}=10: 1)$ as yellow solid $(30.2 \mathrm{mg}, 0.143 \mathrm{mmol}$, 86\%). ${ }^{1} \mathrm{H}$ NMR (400 MHz, $\left.\mathrm{CDCl}_{3}\right) \delta 7.90(\mathrm{~d}, \mathrm{~J}=8.4 \mathrm{~Hz}, 1 \mathrm{H}), 7.18(\mathrm{~d}, \mathrm{~J}=8.3 \mathrm{~Hz}, 1 \mathrm{H}), 7.12(\mathrm{~d}, \mathrm{~J}=8.2$ Hz, 1 H), 6.97 (d, J = 8.2 Hz, 1 H), 5.88 (s, 1 H), 3.64-3.57 (m, 1 H), 3.06-2.98 (m, 1 H), 2.91-2.85 (m, $1 \mathrm{H}), 2.71(\mathrm{~s}, 3 \mathrm{H}), 2.11-2.00(\mathrm{~m}, 1 \mathrm{H}), 1.80-1.70(\mathrm{~m}, 1 \mathrm{H}), 1.40(\mathrm{~d}, \mathrm{~J}=6.3 \mathrm{~Hz}, 3 \mathrm{H}) .{ }^{13} \mathrm{C}$ NMR $(101$ $\left.\mathrm{MHz}_{2} \mathrm{CDCl}_{3}\right) \delta 155.6,140.0,136.7,135.9,127.7,125.2,121.2,116.5,113.2,46.5,30.0,26.6,25.1,22.4$.

\section{1,2,3,4-Tetrahydro-(4,7)-phenanthrolin $6 \mathrm{~g}^{26}$}

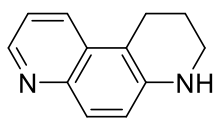

$6 \mathrm{~g}$

Product was isolated via column chromatography $(\mathrm{PE} / \mathrm{EA}=10: 1)$ as yellow oil $(38.2 \mathrm{mg}, 0.208 \mathrm{mmol}$, 93\%). ${ }^{1} \mathrm{H}$ NMR (400 MHz, $\left.\mathrm{CDCl}_{3}\right) \delta 8.60(\mathrm{~d}, \mathrm{~J}=4.1 \mathrm{~Hz}, 1 \mathrm{H}), 8.02(\mathrm{~d}, \mathrm{~J}=8.5 \mathrm{~Hz}, 1 \mathrm{H}), 7.73(\mathrm{~d}, \mathrm{~J}=8.9$ $\mathrm{Hz}, 1 \mathrm{H}), 7.35-7.21(\mathrm{~m}, 1 \mathrm{H}), 6.95$ (d, J = 8.9 Hz, $1 \mathrm{H}), 4.13(\mathrm{~s}, 1 \mathrm{H}), 3.34$ (t, J = 5.4 Hz, $2 \mathrm{H}), 2.97$ (t, J $=6.6 \mathrm{~Hz}, 2 \mathrm{H}), 2.08(\mathrm{q}, \mathrm{J}=6.0 \mathrm{~Hz}, 2 \mathrm{H}) .{ }^{13} \mathrm{C} \mathrm{NMR}\left(101 \mathrm{MHz}, \mathrm{CDCl}_{3}\right) \delta 145.4,143.4,142.2,129.1$, $128.5,128.3,121.6,120.9,110.5,41.5,22.1,21.7$.

\section{5,6-dihydrophenanthridine $6 h^{21}$}<smiles>c1ccc2c(c1)CNc1ccccc1-2</smiles>

Product was isolated via column chromatography $(\mathrm{PE} / \mathrm{EA}=10: 1)$ as yellow oil $(39.8 \mathrm{mg}, 0.218 \mathrm{mmol}$, 87\%). ${ }^{1} \mathrm{H}$ NMR (400 MHz, $\left.\mathrm{CDCl}_{3}\right) \delta$ 7.73-7.67 (m, $\left.2 \mathrm{H}\right), 7.32$ (t, J = 7.6 Hz, $\left.1 \mathrm{H}\right), 7.22(\mathrm{t}, \mathrm{J}=8.1 \mathrm{~Hz}, 1$ H), $7.12(\mathrm{t}, \mathrm{J}=6.7 \mathrm{~Hz}, 2 \mathrm{H}), 6.86(\mathrm{~d}, \mathrm{~J}=7.9 \mathrm{~Hz}, 1 \mathrm{H}), 6.67$ (d, J = 7.9 Hz, 1 H), 4.40 (s, $2 \mathrm{H}), 3.97$ (s, 1 H). ${ }^{13} \mathrm{C} \mathrm{NMR}\left(101 \mathrm{MHz}, \mathrm{CDCl}_{3}\right) \delta 145.7,132.7,132.1,128.8,127.6,127.1,126.0,123.6,122.4,122.0$, $119.2,115.1,46.4$.

\section{1,2,3,4-tetrahydrobenzo[h]quinoline $6 \mathbf{i}^{2}$}<smiles>c1ccc2c3c(ccc2c1)CCCN3</smiles>

$6 i$

Product was isolated via column chromatography $(\mathrm{PE} / \mathrm{EA}=10: 1)$ as yellow oil $(38.0 \mathrm{mg}, 0.208 \mathrm{mmol}$, 83\%). ${ }^{1} \mathrm{H}$ NMR (400 MHz, $\left.\mathrm{CDCl}_{3}\right) \delta$ 7.77-7.73 (m, $\left.1 \mathrm{H}\right), 7.72-7.67(\mathrm{~m}, 1 \mathrm{H})$, 7.43-7.36 (m, $\left.2 \mathrm{H}\right), 7.18$ $(\mathrm{d}, \mathrm{J}=8.3 \mathrm{~Hz}, 1 \mathrm{H}), 7.13(\mathrm{~d}, \mathrm{~J}=8.3 \mathrm{~Hz}, 1 \mathrm{H}), 4.38(\mathrm{~s}, 1 \mathrm{H}), 3.53-3.46(\mathrm{~m}, 2 \mathrm{H}), 2.93(\mathrm{t}, \mathrm{J}=6.4 \mathrm{~Hz}, 2 \mathrm{H})$, 2.18-1.93 (m, $2 \mathrm{H}) .{ }^{13} \mathrm{C} \mathrm{NMR}\left(101 \mathrm{MHz}, \mathrm{CDCl}_{3}\right) \delta 139.0,133.0,128.5,128.5,124.9,124.7,123.2,119.4$, $116.9,115.8,42.4,27.4,22.1$. 
4a,9,9a,10-tetrahydroacridine $6 \mathbf{j}^{2}$<smiles>c1ccc2c(c1)Cc1ccccc1N2</smiles>

6j

Product was isolated via column chromatography $(\mathrm{PE} / \mathrm{EA}=10: 1)$ as white solid $(39.3 \mathrm{mg}, 0.215 \mathrm{mmol}$, 86\%). ${ }^{1} \mathrm{H}$ NMR (400 MHz, $\left.\mathrm{CDCl}_{3}\right) \delta$ 7.19-7.05 (m, $\left.4 \mathrm{H}\right), 6.87$ (t, J = 7.5 Hz, $\left.2 \mathrm{H}\right), 6.8(\mathrm{~d}, \mathrm{~J}=7.8 \mathrm{~Hz}, 2$ $\mathrm{H}), 5.94(\mathrm{~s}, 1 \mathrm{H}), 4.07$ (s, $2 \mathrm{H}) .{ }^{13} \mathrm{C} \mathrm{NMR}\left(101 \mathrm{MHz}, \mathrm{CDCl}_{3}\right) \delta 140.1,128.6,127.0,120.6,120.0,113.4$, 31.4 .

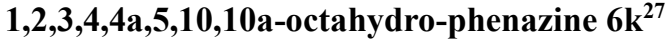<smiles>c1ccc2c(c1)NC1CCCCC1N2</smiles>

$6 k$

Product was isolated via column chromatography $(\mathrm{PE} / \mathrm{EA}=10: 1)$ as yellow oil $(42.8 \mathrm{mg}, 0.228 \mathrm{mmol}$, 91\%). ${ }^{1} \mathrm{H}$ NMR (400 MHz, $\left.\mathrm{CDCl}_{3}\right) \delta$ 6.62-6.53 (m, $\left.2 \mathrm{H}\right), 6.53-6.42(\mathrm{~m}, 2 \mathrm{H}), 3.47$ (s, $\left.4 \mathrm{H}\right), 1.81-1.75(\mathrm{~m}$, $2 \mathrm{H}), 1.71-1.65(\mathrm{~m}, 2 \mathrm{H}), 1.62-1.55(\mathrm{~m}, 2 \mathrm{H}), 1.40-1.32$ (m, $2 \mathrm{H}) .{ }^{13} \mathrm{C} \mathrm{NMR}\left(101 \mathrm{MHz}, \mathrm{CDCl}_{3}\right) \delta 132.4$, $118.3,114.0,50.0,30.6,22.1$.

\section{DFT Computational Study}

\section{Complete Reference for Gaussian 09}

Gaussian 09, Revision D.01, Frisch, M. J.; Trucks, G. W.; Schlegel, H. B.; Scuseria, G. E.; Robb, M. A.; Cheeseman, J. R.; Scalmani, G.; Barone, V.; Mennucci, B.; Petersson, G. A.; Nakatsuji, H.; Caricato, M.; Li, X.; Hratchian, H. P.; Izmaylov, A. F.; Bloino, J.; Zheng, G.; Sonnenberg, J. L.; Hada, M.; Ehara, M.; Toyota, K.; Fukuda, R.; Hasegawa, J.; Ishida, M.; Nakajima, T.; Honda, Y.; Kitao, O.; Nakai, H.; Vreven, T.; Montgomery, Jr., J. A.; Peralta, J. E.; Ogliaro, F.; Bearpark, M.; Heyd, J. J.; Brothers, E.; Kudin, K. N.; Staroverov, V. N.; Kobayashi, R.; Normand, J.; Raghavachari, K.; Rendell, A.; Burant, J. C.; Iyengar, S. S.; Tomasi, J.; Cossi, M.; Rega, N.; Millam, N. J.; Klene, M.; Knox, J. E.; Cross, J. B.; Bakken, V.; Adamo, C.; Jaramillo, J.; Gomperts, R.; Stratmann, R. E.; Yazyev, O.; Austin, A. J.; Cammi, R.; Pomelli, C.; Ochterski, J. W.; Martin, R. L.; Morokuma, K.; Zakrzewski, V. G.; Voth, G. A.; Salvador, P.; Dannenberg, J. J.; Dapprich, S.; Daniels, A. D.; Farkas, Ö.; Foresman, J. B.; Ortiz, J. V.; Cioslowski, J.; Fox, D. J. Gaussian, Inc., Wallingford CT, 2013.

\section{Computational Methods}

All the DFT calculations were carried out with the GAUSSIAN 09 series of programs. Density functional theory B3LYP ${ }^{25}$ with a double- $\zeta$ def2-SVP basis set was used for geometry optimizations. Harmonic frequency calculations were performed for all stationary points to confirm them as a local minima or transition structures and to derive the thermochemical corrections for the enthalpies and free energies. M06- $\mathrm{L}^{26}$ functional with the triple-valence-polarized (def2-TZVP) basis set was employed to calculate the solvation single point energies to give more accurate energy information. The solvent effects were considered by single point calculations on the gas-phase stationary points with a SMD continuum 
solvation model $^{27}$.

\section{Intrinsic Reaction Coordinate (IRC) Calculation of Concerted Hydride/Proton} Transfer Transition State 3-ts

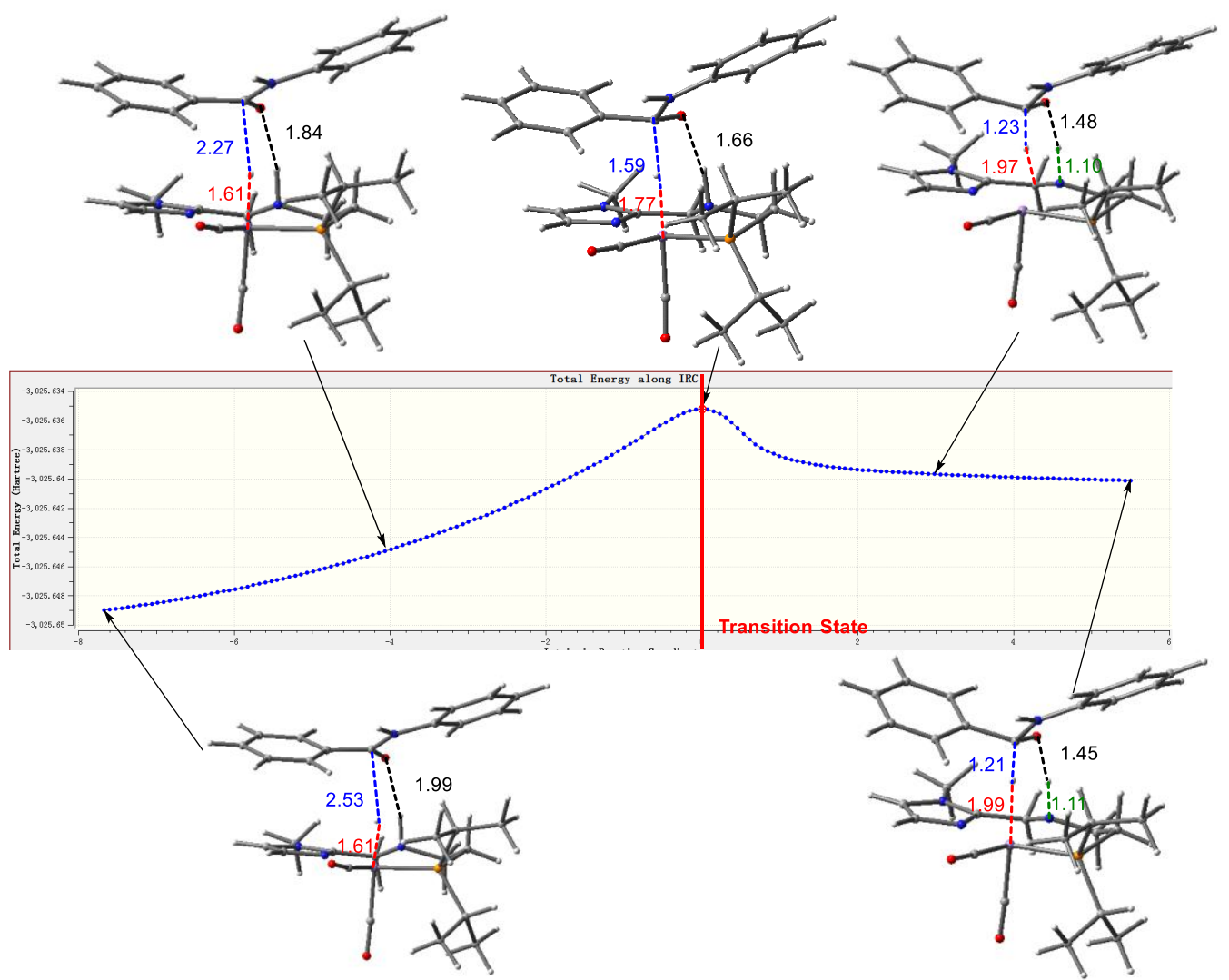

The forward IRC calculation for 3-ts was terminated beforehand. In the terminal geometry of forward IRC calculation, the O-H bond length is $1.45 \AA$ which is shorter than a hydrogen bond. When using the terminus of forward IRC calculation as initial geometry for geometric optimization, the bonding of $\mathrm{H}-\mathrm{O}$ was found in the obtained local minima. This result indicates that the hydrogen transfer of benzanilide $\mathbf{1 a}$ is determined to be a concerted hydride/proton transfer process.

\section{Scheme S2. Proton Dissociation Energy of of Mn-hydride Complexes}

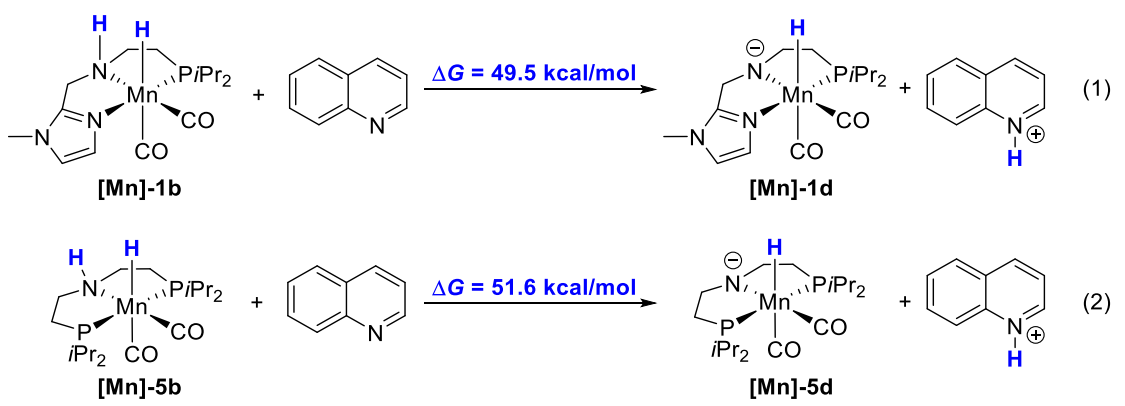

The proton dissociation energy of [Mn]-1b and [Mn]-5b were calculated. As shown in above, the quinoline was employed as proton accepter due to the isolated proton cann't be computationally conducted. The proton dissociation energy of [Mn]-1b is slightly lower than that of [Mn]-5b with 2.1 $\mathrm{kcal} / \mathrm{mol}$, indicating slightly enhanced proton donating ability of [Mn]-1b. These calculated results 
suggest that the reactivity of $\mathrm{Mn}-\mathrm{H}$ species was slightly attributed to its proton dissociation energy, while the hydride dissociation energy play a crucial role.

\section{Ligand Dissociation Assisted $\mathrm{H}_{2}$ Activation}

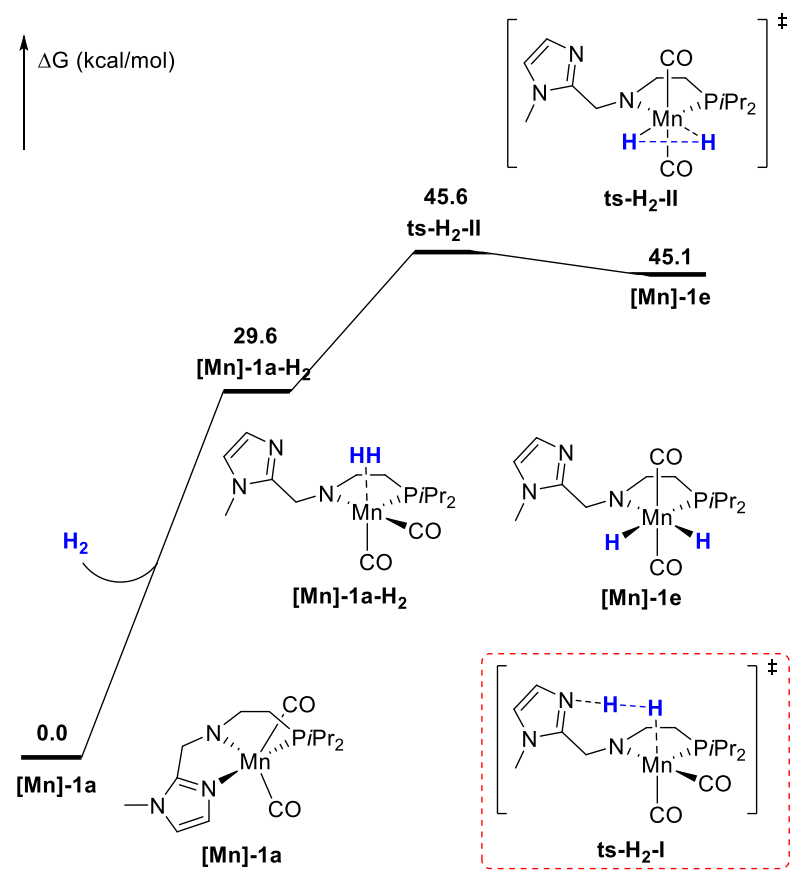

Figure S11. Free energy profile of ligand dissociation followed by $\mathrm{H}_{2}$ coordination.

The hemilability of NNP types of pincer backbones would provide additional options for $\mathrm{H}_{2}$ activation, both heterolytic [with the assistance of dissociated imidazole (via ts-H2-I)] or heterolytic [traditional oxidative addition followed by proton transfer (via ts--H2-II)] are considered. As shown in Figure S11, the $\mathrm{H}_{2}$ coordinated to $\mathrm{Mn}$ center accompanied by the dissociation of imidazole leads to the generation of intermediate [Mn]-1a-H2, and its relative free energy is $5.5 \mathrm{kcal} / \mathrm{mol}$ higher than that of 1-ts in $\mathrm{Mn}-\mathrm{N}$ concerted $\mathrm{H}_{2}$ activation. The following oxidative addition of $\mathrm{H}_{2}$ proceeds via ts-H2-II with an activation

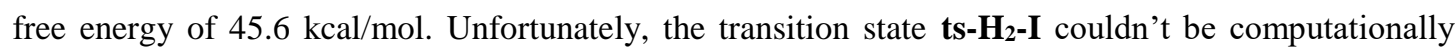
located after much effort. And these results would suggest that the $\mathrm{Mn}-\mathrm{N}$ concerted $\mathrm{H}_{2}$ activation is more energetically favorable. 


\section{Quinoline Assisted $\mathrm{H}_{2}$ Activation}

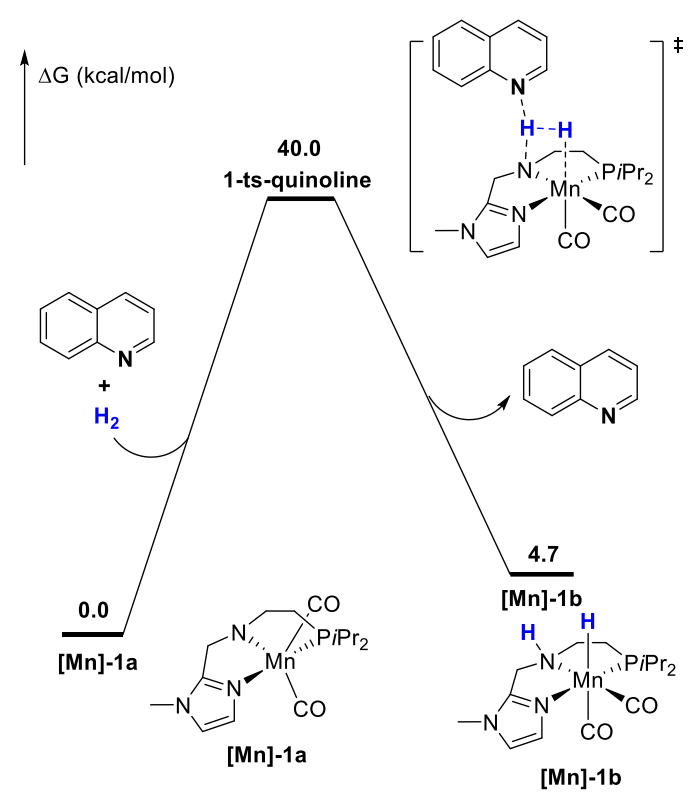

Figure S12. Free energy profile of quinoline assisted $\mathrm{H}_{2}$ acitivation.

Another type of base assisted $\mathrm{H}_{2}$ activation process by quinoline was also conducted. Calculated results showed that the activation free energy of this quinoline assisted $\mathrm{H}_{2}$ activation is up to $40.0 \mathrm{kcal} / \mathrm{mol}$, indicating the base assisted $\mathrm{H}_{2}$ activation is energetically unfavorable.

\section{Isomerization of Hydride Complex [Mn]-1b to [Mn]-1b'}
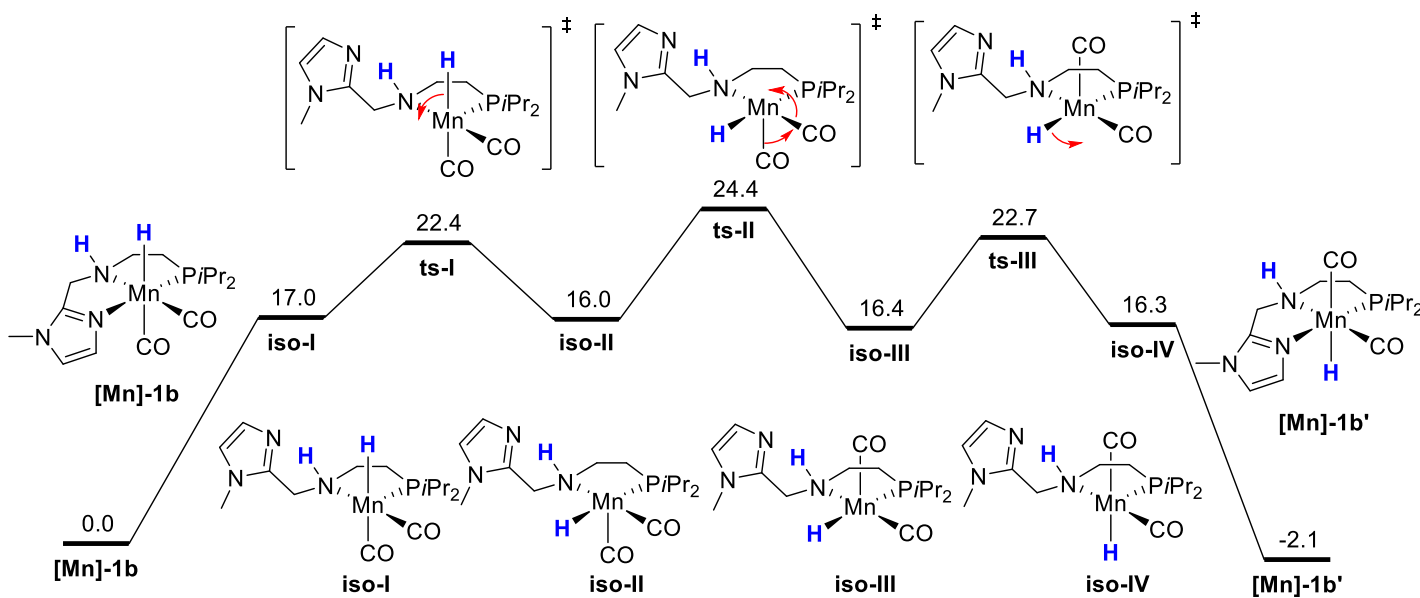

Figure S13. Free energy profiles for the isomerization of hydride [Mn]-1b to [Mn]-1b'.

To illustrate the isomerization of hydride [Mn]-1b to [Mn]-1b', we conducted a detail calculation for the isomeric process, which supported a similar Berry pseudorotation mechanism as the reviewer suggested. As shown in Figure S13, this isomerization is initiated by a dissociation of imidazole to form a penta-coordinated intermediate iso-I. The sequential $\mathrm{Mn}-\mathrm{H}$ and $\mathrm{Mn}-\mathrm{CO}$ bond rotation lead to the conformation variation to generate intermediate iso-IV. Final coordination of imidazole moiety to $\mathrm{Mn}$ center affords the isomer [Mn]-1b'. The overall activation free energy for this isomerization is 24.4 $\mathrm{kcal} / \mathrm{mol}$, which is higher than that of hydrogenation of benzanilide (1a) and quinolone (3a). It indicates 
that the hydrogenation would preferentially proceed in catalytic system rather than isomerization. The activation free energy of isomerization also corroborates the slow isomerization of $[\mathbf{M n}]-\mathbf{1} \mathbf{b}$ at $-35^{\circ} \mathrm{C}$ (42 days long).

\section{Direct 1,3-H Shift of 14}

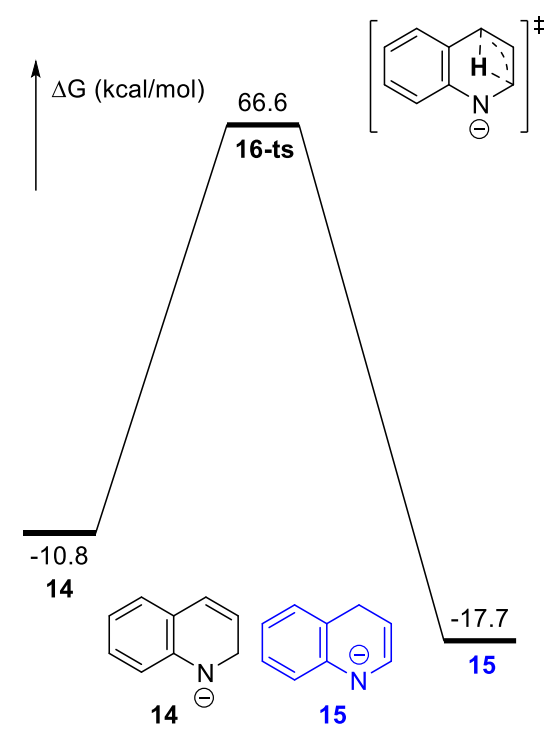

Figure S14. Free energy profile of direct 1,3-H shift of 14.

\section{Disproportionation and Dimerization Pathways}

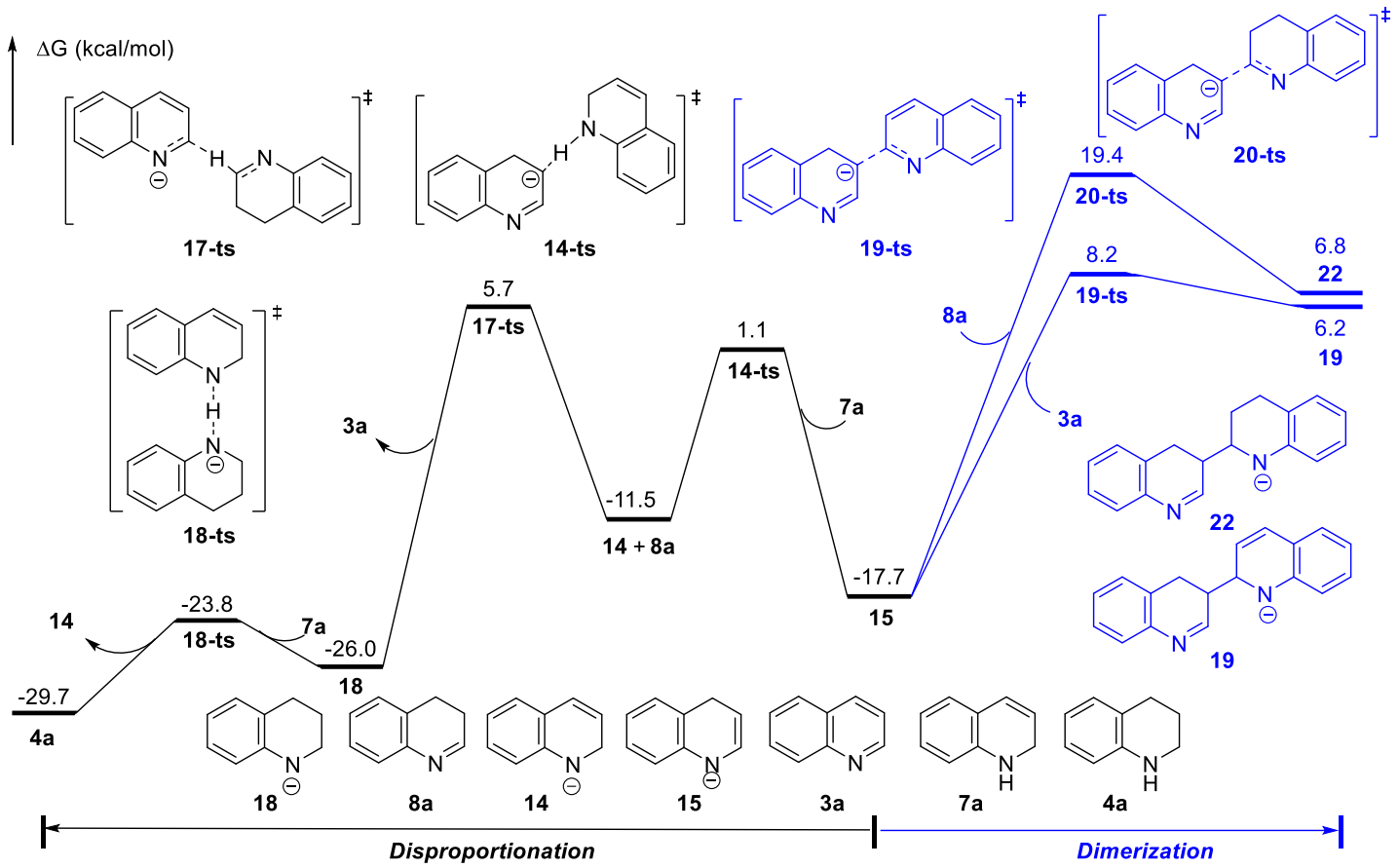

Figure S15. Free energy profiles for the base-promoted disproportionation and dimerization pathways. 


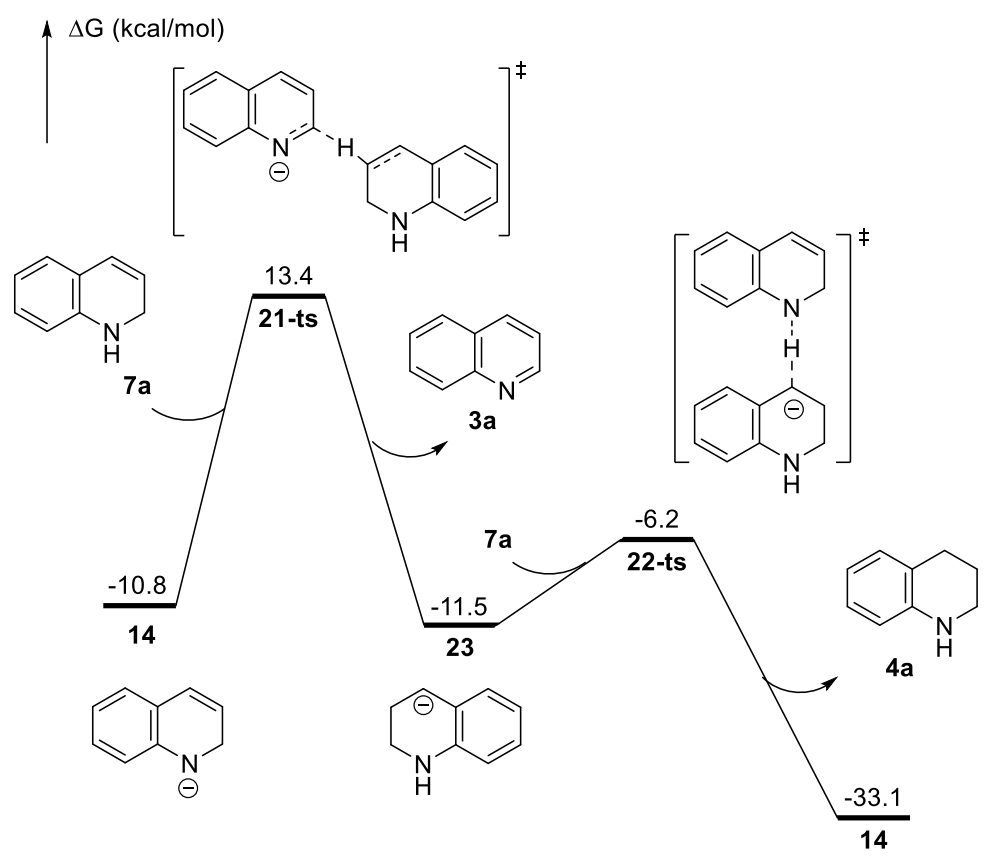

Figure S16. Free energy profiles for the base-promoted disproportionation without the assistance of quinoline 3a.

\section{Frontier Molecular Orbital Analysis}
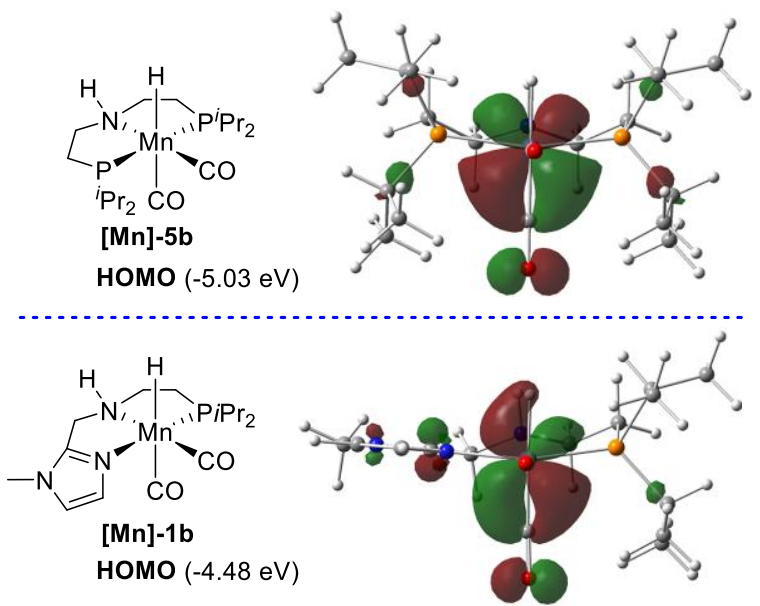

Figure S17. Highest occupied molecular orbital (HOMO) of [Mn]-1b and [Mn]-5b. 


\section{Direct Hydrogenation for $\mathrm{C}=\mathrm{C}$ Bond of $7 \mathrm{a}$}

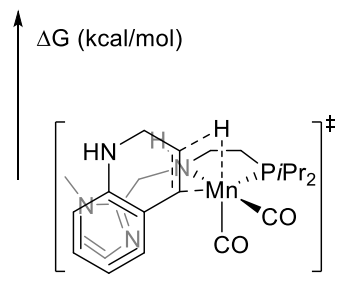

24-ts

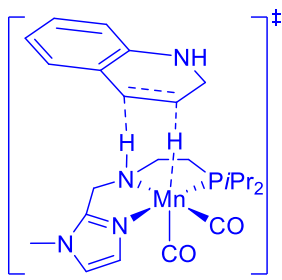

23-ts

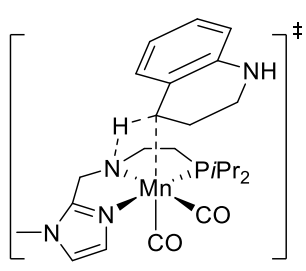

25-ts

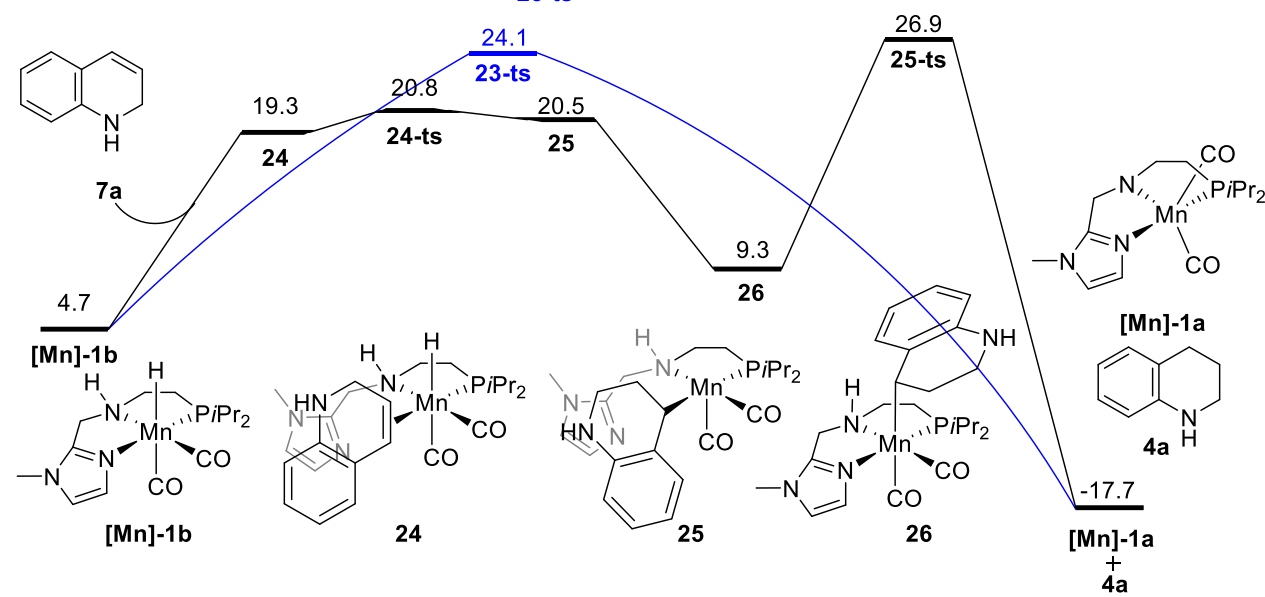

Figure S18. Free energy profiles for the direct hydrogenation for $\mathrm{C}=\mathrm{C}$ bond of $\mathbf{7 a}$.

\section{1,4-Hydrogenation of Quinoline 3a by PNP Mn-H Complex [Mn]-5b}
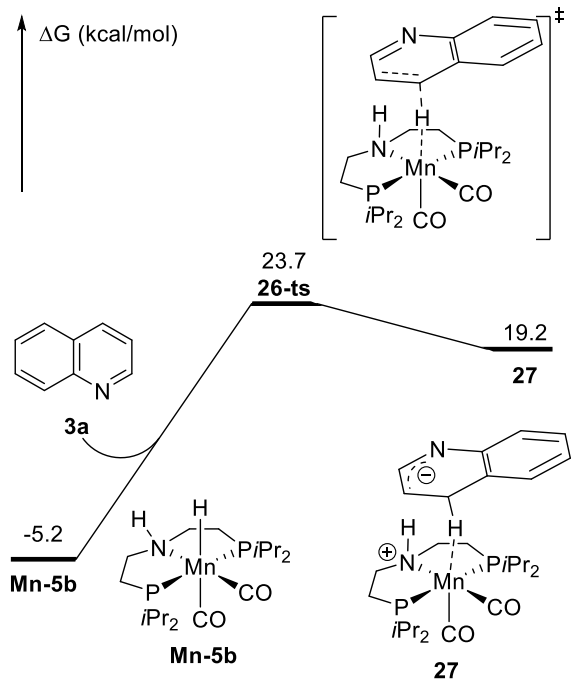

Figure S19. Free energy profiles for the 1,4 hydrogenation of quinoline 3a by PNP Mn-H complex [Mn]5b. 


\section{Optimized Transition State Structures (5-ts and 10-ts)}
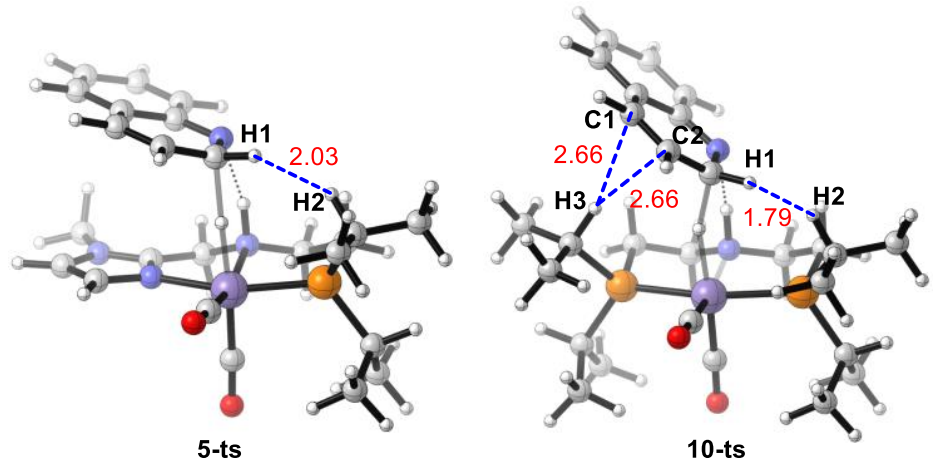

Figure S20. Optimized structures of transition states for hydride trans-fer from NNP- and PNP-Mn hydride complexes to quinoline (3a). Distances are given in angstroms $(\AA)$.

In 10-ts, the distance between $\mathrm{H}_{1}$ and $\mathrm{H}_{3}$ was only $1.79 \AA$, despite the $\mathrm{C}_{1}-\mathrm{H}_{2}$ and $\mathrm{C}_{2}-\mathrm{H}_{2}$ distances being $2.66 \AA$. These data illustrated the apparent steric hindrance be-tween quinoline (3a) and the PNP-pincer ligand in 10-ts. Meanwhile, the distance between $\mathrm{H}_{1}$ and $\mathrm{H}_{2}$ increased to $2.03 \AA$ in 5-ts, indicating that steric repulsion in 5-ts was significantly reduced because a planar imidazole group was involved, which was an important factor in the stabilization of 5-ts.

\section{Intrinsic Reaction Coordinate (IRC) Calculation of Concerted Hydride/Proton Transfer Transition State 15-ts}

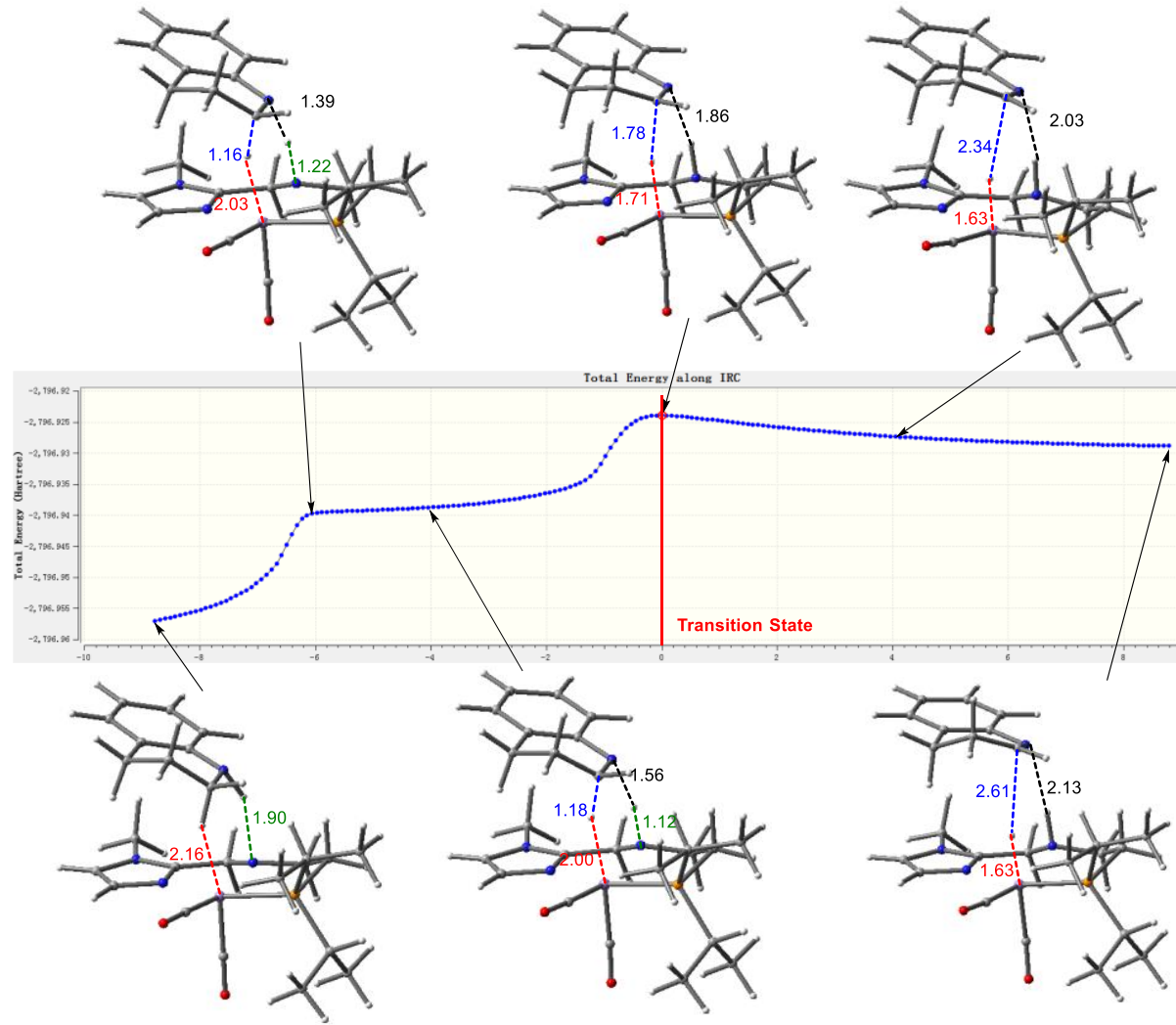

Figure S21. The IRC calculation of transition state 15-ts indicates that the hydrogenation of intermediate 8a undergoes a concerted hydride/proton transfer pathway via 15-ts. 
Absolute Calculation Energies, Enthalpies, and Free Energies

\begin{tabular}{|c|c|c|c|c|c|}
\hline Geometry & $E_{(\text {elec-B3LYP) }}{ }^{1}$ & $G_{(\text {corr-B3LYP })^{2}}$ & $H_{\text {(corr-B3LYP) }^{3}}$ & $E_{(\text {solv, M06-L) }}{ }^{4}$ & $\mathrm{IF}^{5}$ \\
\hline [Mn]-1a & -2392.908229 & 0.33244 & 0.414454 & -2394.030741 & - \\
\hline$[\mathbf{M n}]-\mathbf{1 b}$ & -2394.084405 & 0.354249 & 0.435749 & -2395.217526 & - \\
\hline [Mn]-1b' & -2394.08399 & 0.352902 & 0.435629 & -2395.219565 & - \\
\hline$[\mathrm{Mn}]-\mathbf{1} \mathbf{a}-\mathrm{H}_{2}$ & -2394.048888 & 0.34867 & 0.432741 & -2395.172227 & - \\
\hline [Mn]-5a & -2745.615738 & 0.455596 & 0.549454 & -2746.844418 & - \\
\hline$[M n]-5 b$ & -2746.810956 & 0.478051 & 0.570439 & -2748.047644 & - \\
\hline $\mathbf{H}_{2}$ & -1.17379 & -0.001571 & 0.013266 & -1.170918 & - \\
\hline [Mn]-1c & -2393.326956 & 0.347195 & 0.429594 & -2394.506443 & - \\
\hline$[\mathrm{Mn}]-5 \mathrm{c}$ & -2746.810956 & 0.478051 & 0.570439 & -2748.047644 & - \\
\hline$[\mathbf{M n}]-\mathbf{1 d}$ & -2393.492448 & 0.336858 & 0.418003 & -2394.683058 & - \\
\hline$[M n]-5 d$ & -2746.220834 & 0.461524 & 0.553385 & -2747.510757 & - \\
\hline [Mn]-1e & -2394.020579 & 0.346587 & 0.430159 & -2395.145483 & - \\
\hline iso- $I$ & -2394.053438 & 0.351125 & 0.435813 & -2395.187307 & - \\
\hline iso-II & -2394.053718 & 0.349436 & 0.435374 & -2395.187167 & - \\
\hline iso-III & -2394.051975 & 0.348653 & 0.435212 & -2395.185765 & - \\
\hline iso-IV & -2394.05381 & 0.350178 & 0.435714 & -2395.187473 & - \\
\hline $\mathbf{H}^{-}$ & -0.488729 & -0.01 & 0.00236 & -0.635279 & - \\
\hline${ }^{t} \mathrm{BuO}^{-}$ & -232.874093 & 0.089852 & 0.126134 & -233.187301 & \\
\hline${ }^{t} \mathrm{BuOH}$ & -233.503783 & 0.105833 & 0.142457 & -233.732674 & \\
\hline $1 \mathbf{a}$ & -631.555721 & 0.16965 & 0.221954 & -632.162173 & - \\
\hline 1d & -632.731933 & 0.190387 & 0.244808 & -633.333302 & - \\
\hline $\mathbf{3 a}$ & -401.642158 & 0.10478 & 0.143433 & -402.022843 & - \\
\hline $4 a$ & -404.054082 & 0.14929 & 0.190596 & -404.433145 & - \\
\hline $7 \mathbf{a}$ & -402.830109 & 0.12589 & 0.166723 & -403.209029 & - \\
\hline $\mathbf{8 a}$ & -402.828084 & 0.12619 & 0.165222 & -403.210562 & - \\
\hline $9 a$ & -402.834036 & 0.12428 & 0.166588 & -403.21284 & - \\
\hline 10 & -2795.725743 & 0.4822 & 0.581099 & -2797.239802 & - \\
\hline
\end{tabular}




\begin{tabular}{|c|c|c|c|c|c|}
\hline 11 & -2795.719525 & 0.48361 & 0.581924 & -2797.240073 & - \\
\hline 12 & -2795.736473 & 0.48411 & 0.584516 & -2797.257035 & - \\
\hline 13 & -3148.431539 & 0.605379 & 0.716012 & -3150.051709 & - \\
\hline 14 & -402.2284 & 0.109301 & 0.15079 & -402.680215 & - \\
\hline 15 & -402.240684 & 0.110216 & 0.151249 & -402.69218 & - \\
\hline 18 & -403.445573 & 0.133756 & 0.174608 & -403.899429 & - \\
\hline 19 & -803.882024 & 0.238396 & 0.297244 & -804.700338 & - \\
\hline 22 & -805.095834 & 0.261335 & 0.320914 & -805.917409 & - \\
\hline 23 & -2796.909182 & 0.50252 & 0.60555 & -2798.42571 & - \\
\hline 24 & -2796.920431 & 0.50437 & 0.60842 & -2798.42571 & - \\
\hline 25 & -2796.928434 & 0.50954 & 0.608871 & -2798.448652 & - \\
\hline 26 & -3148.423247 & 0.605508 & 0.715952 & -3150.054211 & - \\
\hline 27 & -403.420411 & 0.133303 & 0.174312 & -403.87054 & - \\
\hline 1-ts & -2394.061256 & 0.34909 & 0.429774 & -2395.181522 & -975.90 \\
\hline 1-ts-quinoline & -2795.683113 & 0.475535 & 0.575714 & -2797.200688 & -129.41 \\
\hline ts-H2-II & -2394.019279 & 0.345198 & 0.428871 & -2395.143355 & -602.83 \\
\hline ts-I & -2394.050281 & 0.349888 & 0.434217 & -2395.177524 & -270.15 \\
\hline ts-II & -2394.048601 & 0.350627 & 0.434372 & -2395.175073 & -50.05 \\
\hline ts-III & -2394.048445 & 0.349223 & 0.434084 & -2395.176316 & -300.65 \\
\hline 2-ts & -2746.773929 & 0.472457 & 0.564724 & -2748.003134 & -664.96 \\
\hline 3-ts & -3025.635221 & 0.545226 & 0.657326 & -3027.372169 & -559.96 \\
\hline 4-ts & -3378.34277 & 0.671821 & 0.79275 & -3380.186228 & -599.81 \\
\hline 5-ts & -2795.72031 & 0.4802 & 0.578969 & -2797.232227 & -567.82 \\
\hline 6-ts & -2795.725066 & 0.47819 & 0.577775 & -2797.232432 & -714.39 \\
\hline 7-ts & -2795.713461 & 0.48024 & 0.578954 & -2797.230963 & -637.41 \\
\hline 8 -ts & -2795.713051 & 0.47932 & 0.577399 & -2797.225605 & -1212.60 \\
\hline 9-ts & -2795.72041 & 0.47924 & 0.57845 & -2797.227554 & -1249.46 \\
\hline 10-ts & -3148.431076 & 0.604049 & 0.714173 & -3150.049968 & -527.27 \\
\hline 11-ts & -3148.428814 & 0.602153 & 0.7125 & -3150.044152 & -1042.78 \\
\hline
\end{tabular}




\begin{tabular}{|c|c|c|c|c|c|}
\hline 12-ts & -803.866846 & 0.230591 & 0.291806 & -804.685766 & -971.19 \\
\hline 13 -ts & -805.102124 & 0.249351 & 0.314897 & -805.904501 & -1134.86 \\
\hline 14-ts & -805.073525 & 0.249219 & 0.314135 & -805.884375 & -943.35 \\
\hline 15-ts & -2796.923935 & 0.50322 & 0.60262 & -2798.436429 & -355.81 \\
\hline 16-ts & -402.094072 & 0.104516 & 0.14365 & -402.552039 & -1611.66 \\
\hline 17-ts & -805.06572 & 0.252997 & 0.315133 & -805.886253 & -790.87 \\
\hline 18-ts & -806.312646 & 0.272074 & 0.338523 & -807.117332 & -1006.77 \\
\hline 19-ts & -803.878364 & 0.236885 & 0.295789 & -804.695567 & -320.49 \\
\hline 20-ts & -805.07381 & 0.257811 & 0.318618 & -805.893697 & -240.09 \\
\hline 21-ts & -2796.906533 & 0.50226 & 0.602416 & -2798.41774 & -598.16 \\
\hline 22-ts & -2796.906012 & 0.50189 & 0.604108 & -2798.422613 & -559.45 \\
\hline 23-ts & -2796.903037 & 0.50234 & 0.602373 & -2798.413427 & -1388.40 \\
\hline 24-ts & -3148.415646 & 0.605216 & 0.71404 & -3150.046778 & -52.69 \\
\hline 25-ts & -805.054464 & 0.253293 & 0.315216 & -805.868829 & -1040.20 \\
\hline 26-ts & -806.2747 & 0.27175 & 0.337967 & -807.08365 & -1034.18 \\
\hline
\end{tabular}

${ }^{1}$ The electronic energy calculated by B3LYP in gas phase. ${ }^{2}$ The thermal correction to Gibbs free energy calculated by B3LYP in gas phase. ${ }^{3}$ The thermal correction to enthalpy calculated by B3LYP in gas phase. ${ }^{4}$ The electronic energy calculated by M06-L in Tetrahydrofuran (THF) solvent. ${ }^{5}$ The B3LYP calculated imaginary frequencies for the transition states.

\section{B3LYP Geometries for All the Optimized Compounds and Transition State}

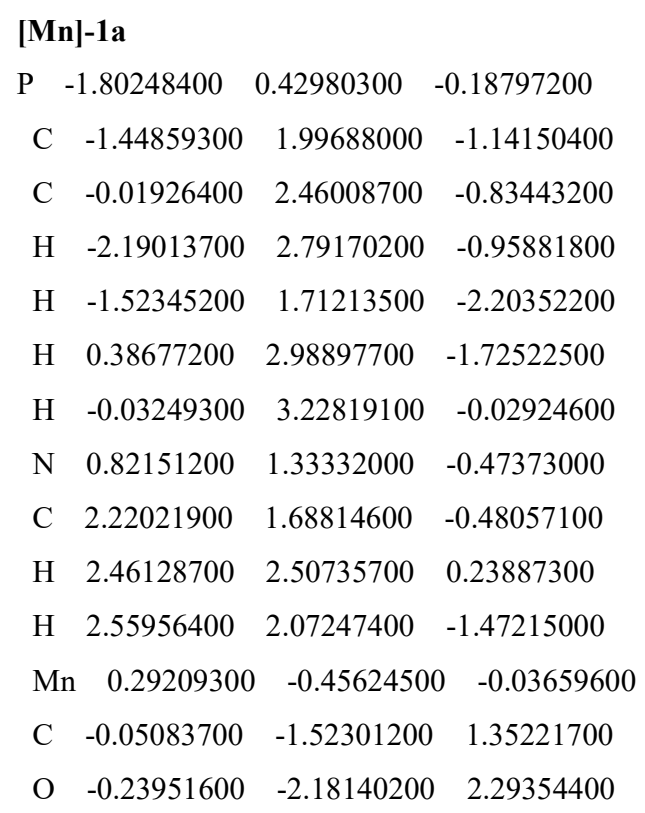




$$
\begin{array}{llll}
\mathrm{H} & -3.48559300 & 1.69993100 & 1.02988300 \\
\mathrm{H} & -1.37339300 & 2.83367800 & 1.65047600 \\
\mathrm{H} & -2.17991300 & 2.26329800 & 3.12522800 \\
\mathrm{H} & -0.78222800 & 1.37076600 & 2.46978300 \\
\mathrm{H} & -4.02636300 & -0.62752700 & 1.73149100 \\
\mathrm{H} & -2.45690000 & -0.78160700 & 2.55512500 \\
\mathrm{H} & -3.67617000 & 0.36164700 & 3.15908500 \\
\mathrm{C} & 2.98055100 & 0.45811800 & -0.12378200 \\
\mathrm{C} & 4.49963500 & -1.07277800 & 0.34439300 \\
\mathrm{H} & 5.48763800 & -1.49347500 & 0.51184700 \\
\mathrm{~N} & 2.31558500 & -0.66324800 & 0.10424200 \\
\mathrm{~N} & 4.31863000 & 0.26268500 & 0.01621000 \\
\mathrm{C} & 3.24715100 & -1.63142200 & 0.39779700 \\
\mathrm{H} & 2.96140300 & -2.65382000 & 0.63250700 \\
\mathrm{C} & 5.35761700 & 1.25104700 & -0.20231600 \\
\mathrm{H} & 6.28241400 & 0.92253100 & 0.29041800 \\
\mathrm{H} & 5.55988800 & 1.39333000 & -1.27663100 \\
\mathrm{H} & 5.05842900 & 2.21677000 & 0.23069900
\end{array}
$$

\section{[Mn]-1b}

P $\quad 1.80822400 \quad-0.31714300 \quad-0.28638900$

C $\quad 1.43385300 \quad-2.05492400 \quad-0.92819800$

$\begin{array}{llll}\text { C } & 0.07038300 & -2.52143700 & -0.41567600\end{array}$

H $\quad 2.21495200 \quad-2.78944800 \quad-0.67839300$

H $\quad 1.40393900 \quad-1.98527700 \quad-2.02899500$

H $\quad-0.22730600 \quad-3.47082300 \quad-0.90625500$

$\mathrm{H} \quad 0.10688700 \quad-2.71179600 \quad 0.66898700$

$\begin{array}{llll}\mathrm{N} & -0.91797200 & -1.46118700 & -0.64529400\end{array}$

C $\quad-2.27353600 \quad-1.71167900 \quad-0.14914100$

$\mathrm{H} \quad-2.19184200 \quad-2.03569800 \quad 0.90519700$

$\mathrm{H} \quad-2.80099700 \quad-2.51655900 \quad-0.69898100$

$\begin{array}{llll}M n & -0.26336400 & 0.50918900 & 0.03021800\end{array}$

$\begin{array}{llll}\text { C } & -0.33004400 & 0.22671200 & 1.80439000\end{array}$

$\begin{array}{llll}\mathrm{O} & -0.32042100 & 0.12012000 & 2.96837400\end{array}$

$\begin{array}{llll}\text { C } & 0.14382600 & 2.22832300 & 0.15797800\end{array}$

$\begin{array}{lllll}\mathrm{O} & 0.38098100 & 3.36822100 & 0.17339000\end{array}$

$\begin{array}{llll}\text { C } & 2.86159200 & 0.33482400 & -1.71682900\end{array}$

$\begin{array}{llll}\text { C } & 4.07000500 & -0.52866800 & -2.10201300\end{array}$

$\begin{array}{llll}\text { C } & 3.26693300 & 1.80626000 & -1.55576200\end{array}$

$\begin{array}{llll}\mathrm{H} & 2.11895900 & 0.29272900 & -2.53224200\end{array}$

H $\quad 3.80937600 \quad-1.58583500 \quad-2.26649100$

H $\quad 4.51712200 \quad-0.15344700 \quad-3.03852600$

H $\quad 4.86123000 \quad-0.49263400 \quad-1.33541500$

$\begin{array}{llll}\mathrm{H} & 2.41975900 & 2.44180400 & -1.26487100\end{array}$

H $\quad 4.06463700 \quad 1.93027500 \quad-0.80568000$

$$
\begin{array}{llll}
\mathrm{H} & 3.66398700 & 2.18957400 & -2.51116800 \\
\mathrm{C} & 3.05851300 & -0.61031100 & 1.10706600 \\
\mathrm{C} & 2.64461800 & -1.74504000 & 2.05532600 \\
\mathrm{C} & 3.28171000 & 0.68562100 & 1.90287400 \\
\mathrm{H} & 4.00800400 & -0.89850200 & 0.62341400 \\
\mathrm{H} & 2.55056400 & -2.71594400 & 1.54455600 \\
\mathrm{H} & 3.40896700 & -1.86897600 & 2.84144200 \\
\mathrm{H} & 1.69337100 & -1.52030800 & 2.56063400 \\
\mathrm{H} & 3.58926500 & 1.52768600 & 1.26698500 \\
\mathrm{H} & 2.36278500 & 0.98476800 & 2.42893300 \\
\mathrm{H} & 4.06960400 & 0.53388100 & 2.66050700 \\
\mathrm{C} & -2.99433600 & -0.40164800 & -0.23053900 \\
\mathrm{C} & -4.48266200 & 1.22044800 & -0.33143800 \\
\mathrm{H} & -5.46010700 & 1.68960300 & -0.40795900 \\
\mathrm{~N} & -2.30720100 & 0.72830600 & -0.16218700 \\
\mathrm{~N} & -4.33173900 & -0.15513000 & -0.34017900 \\
\mathrm{C} & -3.21827800 & 1.75050900 & -0.22684000 \\
\mathrm{H} & -2.90961100 & 2.79274700 & -0.20019700 \\
\mathrm{C} & -5.40370500 & -1.13242200 & -0.37245700 \\
\mathrm{H} & -6.30040300 & -0.67127100 & -0.80754400 \\
\mathrm{H} & -5.12165800 & -1.99084200 & -0.99911600 \\
\mathrm{H} & -5.65069600 & -1.49787100 & 0.63793800 \\
\mathrm{H} & -0.95787500 & -1.23409800 & -1.63942200 \\
\mathrm{H} & -0.14151000 & 0.81363500 & -1.56988700
\end{array}
$$

[Mn]-1b'

$\begin{array}{llll}\mathrm{P} & -1.79396900 & 0.38596000 & -0.08989600\end{array}$

$\begin{array}{llll}\text { C } & -1.45343800 & 1.98598600 & -1.02699200\end{array}$

C $\quad-0.05662500 \quad 2.49422800 \quad-0.67935400$

$\mathrm{H} \quad-2.20249500 \quad 2.77363800 \quad-0.84573800$

$\begin{array}{llll}\mathrm{H} & -1.50629400 & 1.73774500 & -2.10107300\end{array}$

H $\quad 0.21328500 \quad 3.36752400 \quad-1.30686600$

$\mathrm{H} \quad-0.02959800 \quad 2.82353500 \quad 0.36893100$

$\begin{array}{lllll}\mathrm{N} & 0.93956000 & 1.41477800 & -0.81075400\end{array}$

$\begin{array}{llll}\text { C } & 2.24572600 & 1.74441200 & -0.21898200\end{array}$

$\begin{array}{llll}\mathrm{H} & 2.04091600 & 2.19585900 & 0.76837100\end{array}$

H $\quad 2.82116600 \quad 2.48130200 \quad-0.81239300$

$\begin{array}{llll}\text { Mn } & 0.26392100 & -0.49060300 & 0.07116700\end{array}$

$\begin{array}{llll}\text { C } & 0.12507700 & -1.40564800 & -1.46874300\end{array}$

$\begin{array}{llll}\text { O } & -0.03404100 & -2.01569300 & -2.45194900\end{array}$

$\begin{array}{llll}\text { C } & -0.13343400 & -1.86417600 & 1.11857300\end{array}$

$\begin{array}{llll}\text { O } & -0.36805500 & -2.73671800 & 1.85242400\end{array}$

$\begin{array}{llll}\text { C } & -3.08422800 & -0.39608400 & -1.23345300\end{array}$

$\begin{array}{llll}\text { C } & -4.32722000 & 0.45169600 & -1.53202700\end{array}$

$\begin{array}{llll}\text { C } & -3.46948900 & -1.81994900 & -0.80848500\end{array}$ 


$$
\begin{array}{llll}
\mathrm{H} & -2.49670000 & -0.48920400 & -2.16435000 \\
\mathrm{H} & -4.07889000 & 1.46879000 & -1.87397600 \\
\mathrm{H} & -4.92731100 & -0.02291600 & -2.32727700 \\
\mathrm{H} & -4.98248800 & 0.54324100 & -0.65041600 \\
\mathrm{H} & -2.58658400 & -2.43235200 & -0.57559000 \\
\mathrm{H} & -4.12879100 & -1.81854000 & 0.07423000 \\
\mathrm{H} & -4.02037000 & -2.31997800 & -1.62293700 \\
\mathrm{C} & -2.78789900 & 0.93450400 & 1.42445700 \\
\mathrm{C} & -2.19290400 & 2.16518500 & 2.12077800 \\
\mathrm{C} & -2.92314300 & -0.22095700 & 2.42776400 \\
\mathrm{H} & -3.79126700 & 1.20265700 & 1.05094000 \\
\mathrm{H} & -2.20143000 & 3.06241800 & 1.48207800 \\
\mathrm{H} & -2.78254300 & 2.40580800 & 3.02178900 \\
\mathrm{H} & -1.15697500 & 1.96757700 & 2.43870200 \\
\mathrm{H} & -3.34180600 & -1.13212200 & 1.97770900 \\
\mathrm{H} & -1.94272400 & -0.48525200 & 2.85062900 \\
\mathrm{H} & -3.58539200 & 0.07665300 & 3.25876800 \\
\mathrm{C} & 2.98592500 & 0.46367200 & -0.01929900 \\
\mathrm{C} & 4.49906100 & -1.09562300 & 0.34799800 \\
\mathrm{H} & 5.48544300 & -1.53855000 & 0.45846000 \\
\mathrm{~N} & 2.31078500 & -0.65101100 & 0.20776600 \\
\mathrm{~N} & 4.33136400 & 0.24597100 & 0.05267000 \\
\mathrm{C} & 3.23788300 & -1.63611300 & 0.43336600 \\
\mathrm{H} & 2.94357700 & -2.66262500 & 0.63744200 \\
\mathrm{C} & 5.38609900 & 1.23294000 & -0.08264200 \\
\mathrm{H} & 6.34353400 & 0.71739600 & -0.23453000 \\
\mathrm{H} & 5.20300400 & 1.87839400 & -0.95449200 \\
\mathrm{H} & 5.46757100 & 1.86614300 & 0.81605000 \\
\mathrm{H} & 1.06892900 & 1.19229600 & -1.79861900 \\
\mathrm{H} & 0.32218500 & 0.36293300 & 1.46408800 \\
\mathrm{I} & -1 \mathrm{a}-\mathrm{s} 2 & &
\end{array}
$$

\section{[Mn]-1a-H}

$\begin{array}{llll}\mathrm{P} & -1.73018300 & -0.68916700 & 0.06687000\end{array}$

$$
\begin{array}{llll}
\mathrm{C} & -0.51121500 & -1.83805100 & -0.72733500 \\
\mathrm{C} & 0.54560500 & -0.99707700 & -1.43659000 \\
\mathrm{H} & -0.98306800 & -2.56882400 & -1.40356200 \\
\mathrm{H} & -0.03614600 & -2.39985600 & 0.09345900 \\
\mathrm{H} & 1.43658600 & -1.62002600 & -1.62561700 \\
\mathrm{H} & 0.18503500 & -0.68646800 & -2.43758300 \\
\mathrm{~N} & 0.91080400 & 0.19735700 & -0.65344000 \\
\mathrm{C} & 2.25639300 & 0.62992500 & -1.07251000 \\
\mathrm{H} & 2.46114000 & 1.63023900 & -0.67025800 \\
\mathrm{H} & 2.31140100 & 0.70951800 & -2.17386500 \\
\mathrm{Mn} & -0.30611900 & 1.18546500 & 0.37250300 \\
\mathrm{C} & -1.36443400 & 2.34606100 & -0.47608100
\end{array}
$$

$$
\begin{array}{cccc}
\mathrm{O} & -1.98177200 & 3.06893700 & -1.13879800 \\
\mathrm{C} & 0.84698100 & 2.49387500 & 0.82388300 \\
\mathrm{O} & 1.52858600 & 3.35740700 & 1.17942900 \\
\mathrm{C} & -2.26956700 & -1.66825300 & 1.58896500 \\
\mathrm{C} & -2.90860500 & -3.02612600 & 1.26907300 \\
\mathrm{C} & -3.11574300 & -0.87565000 & 2.59501800 \\
\mathrm{H} & -1.28857200 & -1.85320300 & 2.06340100 \\
\mathrm{H} & -2.29535900 & -3.63072900 & 0.58353900 \\
\mathrm{H} & -3.03845400 & -3.60965700 & 2.19602900 \\
\mathrm{H} & -3.90761000 & -2.90959400 & 0.81919200 \\
\mathrm{H} & -2.68356700 & 0.10976800 & 2.81902300 \\
\mathrm{H} & -4.14486000 & -0.72022700 & 2.23727500 \\
\mathrm{H} & -3.18516900 & -1.43233000 & 3.54426300 \\
\mathrm{C} & -3.21014800 & -0.67433600 & -1.10071200 \\
\mathrm{C} & -2.78934600 & -0.31915900 & -2.53517200 \\
\mathrm{C} & -4.32921600 & 0.26034800 & -0.61814900 \\
\mathrm{H} & -3.59270200 & -1.70994200 & -1.10388300 \\
\mathrm{H} & -2.07071400 & -1.03776500 & -2.95527400 \\
\mathrm{H} & -3.67630100 & -0.32300200 & -3.19032500 \\
\mathrm{H} & -2.34427100 & 0.68595300 & -2.59102400 \\
\mathrm{H} & -4.71224100 & -0.01116000 & 0.37547500 \\
\mathrm{H} & -3.99214100 & 1.30714500 & -0.58015500 \\
\mathrm{H} & -5.17894900 & 0.21429500 & -1.31957300 \\
\mathrm{C} & 3.36068200 & -0.31532000 & -0.67728400 \\
\mathrm{C} & 4.83148800 & -1.39693400 & 0.57090300 \\
\mathrm{H} & 5.39966100 & -1.66773900 & 1.45803600 \\
\mathrm{~N} & 3.94384200 & -1.18331100 & -1.48715000 \\
\mathrm{~N} & 3.86898600 & -0.40969700 & 0.59701300 \\
\mathrm{C} & 4.85950500 & -1.85882000 & -0.72571200 \\
\mathrm{H} & 5.49468100 & -2.63932800 & -1.14380400 \\
\mathrm{C} & 3.46308100 & 0.36040700 & 1.75875000 \\
\mathrm{H} & 4.05312300 & 0.03942100 & 2.62727000 \\
\mathrm{H} & 3.62888300 & 1.43699400 & 1.60218600 \\
\mathrm{H} & 2.39438000 & 0.20473200 & 1.96784700 \\
\mathrm{H} & -1.33754300 & 1.65827500 & 1.58854400 \\
\mathrm{H} & -0.72420600 & 1.15788000 & 1.96148500
\end{array}
$$$$
\text { [Mn]-5a }
$$$$
\text { P } \quad 2.28059400 \quad-0.10409900 \quad-0.23930100
$$$$
\text { C } \quad 2.45665900 \quad-1.12519200 \quad-1.77910200
$$$$
\begin{array}{llll}
\text { C } & 1.18761900 & -1.96149300 & -1.95586800
\end{array}
$$$$
\text { H } \quad 3.36630100 \quad-1.74763700 \quad-1.78667200
$$$$
\mathrm{H} \quad 2.54030700 \quad-0.40302100 \quad-2.60748900
$$$$
\mathrm{H} \quad 1.05186900 \quad-2.17509500 \quad-3.03679400
$$$$
\mathrm{H} \quad 1.32051800 \quad-2.95957000 \quad-1.48623500
$$ 


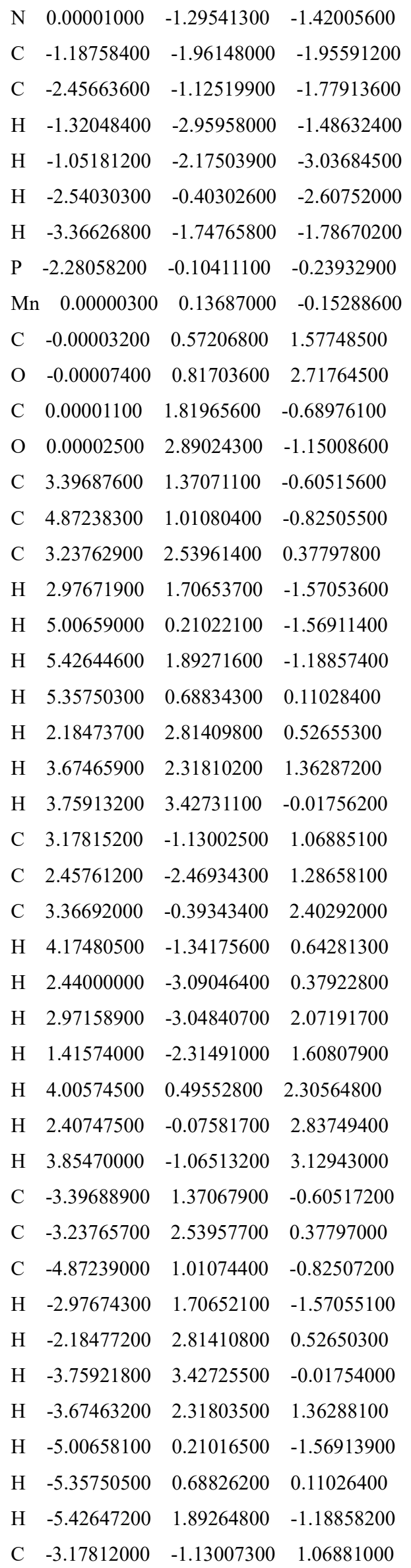

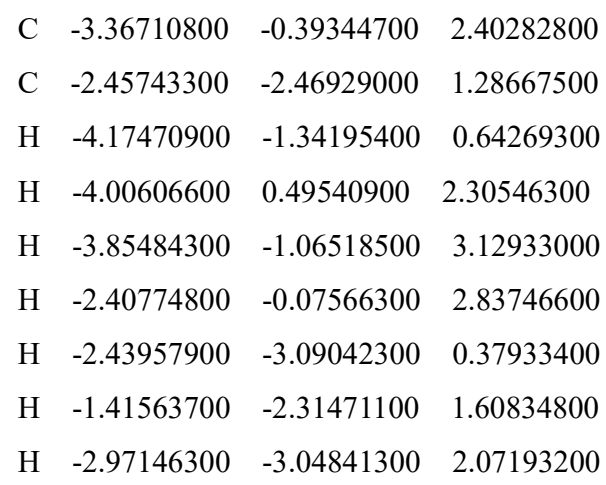




$$
\begin{array}{cccc}
\mathrm{H} & -5.21892500 & 1.23166600 & -0.73595300 \\
\mathrm{H} & -4.81916200 & 2.35913200 & -2.04069200 \\
\mathrm{C} & -3.48303000 & -0.59923800 & 0.96923000 \\
\mathrm{C} & -3.23897700 & 0.04076700 & 2.34545500 \\
\mathrm{C} & -3.46265100 & -2.13056300 & 1.08722400 \\
\mathrm{H} & -4.48288200 & -0.29864800 & 0.61144500 \\
\mathrm{H} & -3.22358700 & 1.13915000 & 2.30981200 \\
\mathrm{H} & -4.03488400 & -0.26069000 & 3.04742100 \\
\mathrm{H} & -2.27781400 & -0.28962700 & 2.76687800 \\
\mathrm{H} & -3.73097700 & -2.63504200 & 0.14659600 \\
\mathrm{H} & -2.47857800 & -2.49924500 & 1.41311500 \\
\mathrm{H} & -4.19754700 & -2.45154000 & 1.84477100 \\
\mathrm{H} & -0.00003300 & 1.24699600 & -1.04790600 \\
\text { H2 } & & &
\end{array}
$$

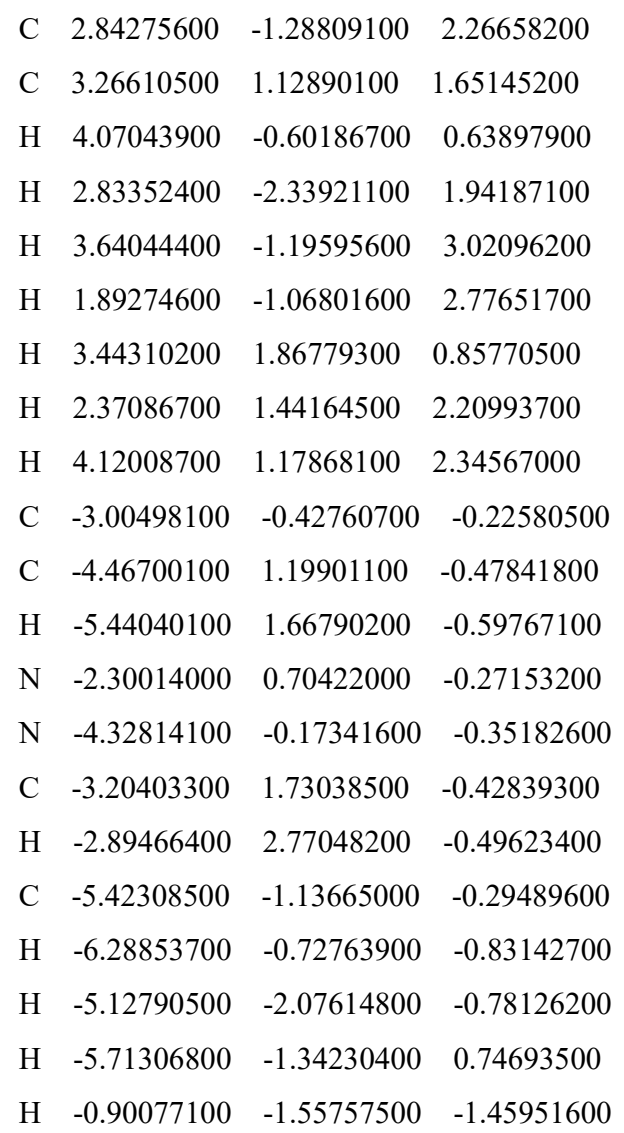




$$
\begin{aligned}
& \text { C } \quad 4.49295700 \quad 1.40800300 \quad-1.62620800 \\
& \text { C } \quad 3.09856500 \quad 2.80202200 \quad-0.03551900 \\
& \mathrm{H} \quad 2.39948400 \quad 1.86720000 \quad-1.83899500 \\
& \mathrm{H} \quad 4.51923600 \quad 0.60701500 \quad-2.37989200 \\
& \mathrm{H} \quad 4.82200100 \quad 2.33429500 \quad-2.12407100 \\
& \text { H } \quad 5.24373700 \quad 1.17588300 \quad-0.85465400 \\
& \text { H } \quad 2.11797500 \quad 2.97604100 \quad 0.42931800 \\
& \text { H } \quad 3.83601500 \quad 2.65970900 \quad 0.76914800 \\
& \mathrm{H} \quad 3.38201100 \quad 3.72238500 \quad-0.57034400 \\
& \begin{array}{llll}
\text { C } & 3.50360700 & -0.57760300 & 1.01402300
\end{array} \\
& \begin{array}{llll}
\text { C } & 3.50130900 & -2.10779700 & 1.15029800
\end{array} \\
& \text { C } \quad 3.22630700 \quad 0.09216700 \quad 2.36988900 \\
& \text { H } \quad 4.50291600 \quad-0.26719100 \quad 0.66454500 \\
& \text { H } \quad 3.79730200 \quad-2.62281700 \quad 0.22466900 \\
& \text { H } \quad 4.22992700 \quad-2.39942600 \quad 1.92345400 \\
& \text { H } \quad 2.52189200 \quad-2.49338400 \quad 1.47110400 \\
& \mathrm{H} \quad 3.19214900 \quad 1.18884800 \quad 2.31243800 \\
& \text { H } \quad 2.27517800 \quad-0.25398500 \quad 2.80150900 \\
& \text { H } \quad 4.02497900 \quad-0.17620900 \quad 3.07964300 \\
& \text { C } \quad-3.09922200 \quad 1.62115400 \quad-1.01739400 \\
& \begin{array}{llll}
\text { C } & -3.09851800 & 2.80199700 & -0.03554000
\end{array} \\
& \begin{array}{llll}
\text { C } & -4.49296600 & 1.40798100 & -1.62618600
\end{array} \\
& \text { H } \quad-2.39949400 \quad 1.86715500 \quad-1.83903000 \\
& \text { H } \quad-2.11790900 \quad 2.97601400 \quad 0.42926100 \\
& \mathrm{H} \quad-3.38197900 \quad 3.72235900 \quad-0.57035800 \\
& \mathrm{H} \quad-3.83594000 \quad 2.65969000 \quad 0.76915600 \\
& \mathrm{H} \quad-4.51927200 \quad 0.60699300 \quad-2.37986900 \\
& \mathrm{H} \quad-5.24372900 \quad 1.17586800 \quad-0.85461300 \\
& \text { H } \quad-4.82201300 \quad 2.33427500 \quad-2.12404300 \\
& \begin{array}{llll}
\text { C } & -3.50362400 & -0.57760400 & 1.01401500
\end{array} \\
& \begin{array}{llll}
\text { C } & -3.22634400 & 0.09218800 & 2.36987300
\end{array} \\
& \text { C } \quad-3.50135200 \quad-2.10779600 \quad 1.15031200 \\
& \mathrm{H} \quad-4.50292500 \quad-0.26718600 \quad 0.66451900 \\
& \mathrm{H} \quad-3.19216100 \quad 1.18886800 \quad 2.31240000 \\
& \text { H } \quad-4.02504100 \quad-0.17615400 \quad 3.07961000 \\
& \mathrm{H} \quad-2.27523600 \quad-0.25397700 \quad 2.80152500 \\
& \text { H } \quad-3.79734800 \quad-2.62282500 \quad 0.22469000 \\
& \mathrm{H} \quad-2.52194500 \quad-2.49339400 \quad 1.47113100 \\
& \text { H } \quad-4.22998100 \quad-2.39940100 \quad 1.92346700
\end{aligned}
$$

\section{[Mn]-1d}

P $\quad 1.75474200 \quad-0.35221800 \quad-0.29989300$

$\begin{array}{llll}\text { C } & 1.38728600 & -2.09698100 & -0.89472300\end{array}$

$\begin{array}{llll}\text { C } & 0.01026200 & -2.49953600 & -0.34979600\end{array}$

H $\quad 2.17978000 \quad-2.82489600 \quad-0.65015200$

$$
\begin{aligned}
& \text { H } \quad 1.31539200 \quad-2.02640700 \quad-1.99262300 \\
& \text { Н } \quad-0.30466200 \quad-3.45310300 \quad-0.85380600 \\
& \text { H } \quad 0.11263800 \quad-2.78473700 \quad 0.73837600 \\
& \mathrm{~N} \quad-0.89812700 \quad-1.43013300 \quad-0.57912400 \\
& \text { C } \quad-2.21108300-1.70190500 \quad-0.12150700 \\
& \text { H } \quad-2.27528500 \quad-2.03413900 \quad 0.96577700 \\
& \mathrm{H} \quad-2.74366500 \quad-2.52183100 \quad-0.68198600 \\
& \begin{array}{llll}
\text { Mn } & -0.27045300 & 0.51232700 & 0.04576400
\end{array} \\
& \begin{array}{llll}
\text { C } & -0.30730700 & 0.26484800 & 1.82250400
\end{array} \\
& \begin{array}{llll}
\text { O } & -0.22203400 & 0.24707200 & 2.99719200
\end{array} \\
& \begin{array}{llll}
\text { C } & 0.14510700 & 2.22389600 & 0.13717400
\end{array} \\
& \begin{array}{llll}
\text { O } & 0.40996600 & 3.36923400 & 0.12039900
\end{array} \\
& \begin{array}{llll}
\text { C } & 2.80431800 & 0.28172500 & -1.75410700
\end{array} \\
& \begin{array}{llll}
\text { C } & 3.99858100 & -0.59250800 & -2.15784900
\end{array} \\
& \begin{array}{llll}
\text { C } & 3.22184300 & 1.75203200 & -1.62441700
\end{array} \\
& \text { H } \quad 2.04369200 \quad 0.23222800 \quad-2.55177200 \\
& \text { H } 3.71909300 \quad-1.64761800 \quad-2.30266500 \\
& \text { H } \quad 4.43586100 \quad-0.23298800 \quad-3.10772000 \\
& \text { H } \quad 4.80456100 \quad-0.55987500 \quad-1.40474300 \\
& \text { H } \quad 2.38423700 \quad 2.38987700 \quad-1.31030400 \\
& \text { H } \quad 4.04249400 \quad 1.88040200 \quad-0.89822200 \\
& \text { H } \quad 3.59370100 \quad 2.12935300 \quad-2.59489400 \\
& \begin{array}{llll}
\text { C } & 3.05669300 & -0.59778000 & 1.07259600
\end{array} \\
& \begin{array}{llll}
\text { C } & 2.67224300 & -1.71378700 & 2.05423300
\end{array} \\
& \begin{array}{llll}
\text { C } & 3.27719900 & 0.71451100 & 1.84155000
\end{array} \\
& \text { H } \quad 4.00332500 \quad-0.88540800 \quad 0.57971700 \\
& \mathrm{H} \quad 2.57420100 \quad-2.69365800 \quad 1.56314700 \\
& \text { H } \quad 3.44641000 \quad-1.81118100 \quad 2.83798100 \\
& \text { H } \quad 1.71897000 \quad-1.48789700 \quad 2.55506500 \\
& \text { H } \quad 3.54319100 \quad 1.55358800 \quad 1.18275800 \\
& \text { H } \quad 2.36364100 \quad 0.99971900 \quad 2.38434000 \\
& \mathrm{H} \quad 4.09041500 \quad 0.59606400 \quad 2.58168200 \\
& \text { C } \quad-2.96708100 \quad-0.41597800 \quad-0.23561000 \\
& \begin{array}{llll}
\text { C } & -4.49697600 & 1.18318500 & -0.37091800
\end{array} \\
& \mathrm{H} \quad-5.48392800 \quad 1.62824900 \quad-0.47233200 \\
& \mathrm{~N} \quad-2.31235600 \quad 0.73123900 \quad-0.16540500 \\
& \mathrm{~N} \quad-4.31044200 \quad-0.19270200 \quad-0.36536500 \\
& \text { C } \quad-3.24245000 \quad 1.73376600 \quad-0.25034700 \\
& \mathrm{H} \quad-2.95046500 \quad 2.78162500 \quad-0.22770900 \\
& \text { C } \quad-5.35289200 \quad-1.19200600 \quad-0.39845800 \\
& \mathrm{H} \quad-6.17329300 \quad-0.85884400 \quad-1.05294400 \\
& \text { H } \quad-4.94734700 \quad-2.13186300 \quad-0.79798900 \\
& \mathrm{H} \quad-5.76591000 \quad-1.39190500 \quad 0.60636400 \\
& \mathrm{H} \quad-0.11828700 \quad 0.88017700 \quad-1.53446500
\end{aligned}
$$




\section{[Mn]-5d}

P $\quad 2.20132000 \quad 0.06721200 \quad-0.33046800$

$\begin{array}{llll}\text { C } & 2.40122400 & -0.97762900 & -1.86818200\end{array}$

C $\quad 1.18534800 \quad-1.91005900 \quad-1.93121600$

Н $\quad 3.36579400 \quad-1.51044400 \quad-1.91597600$

H $\quad 2.34900100 \quad-0.28167300 \quad-2.72149400$

Н $\quad 1.17986600 \quad-2.41481900 \quad-2.93178300$

Н $\quad 1.33577000 \quad-2.75172200 \quad-1.19541200$

$\mathrm{N} \quad 0.00000100 \quad-1.14422900 \quad-1.71877000$

C $\quad-1.18533200-1.91007400 \quad-1.93122200$

C $\quad-2.40121800 \quad-0.97766000 \quad-1.86818800$

H $\quad-1.33574400 \quad-2.75173800 \quad-1.19542100$

H $\quad-1.17984000 \quad-2.41482900 \quad-2.93179100$

$\mathrm{H} \quad-2.34900800 \quad-0.28171100 \quad-2.72150600$

H $\quad-3.36578100 \quad-1.51048700 \quad-1.91597300$

P $\quad-2.20131900 \quad 0.06719600 \quad-0.33048100$

$\begin{array}{llll}\text { Mn } & -0.00000100 & 0.00485300 & 0.11503700\end{array}$

$\begin{array}{llll}\text { C } & 0.00000800 & -1.42437700 & 1.18707000\end{array}$

$\begin{array}{llll}\text { O } & -0.00002400 & -2.30051100 & 1.97210300\end{array}$

C $\quad-0.00000100 \quad 1.18368200 \quad 1.42537700$

$\begin{array}{llll}\mathrm{O} & -0.00001200 & 2.01374200 & 2.25850400\end{array}$

C $\quad 2.97778800 \quad 1.70087400 \quad-0.91117500$

C $\quad 4.38997800 \quad 1.59501000 \quad-1.50124300$

$\begin{array}{llll}\text { C } & 2.89591200 & 2.83298900 & 0.11983900\end{array}$

H $\quad 2.27499500 \quad 1.95146400 \quad-1.72432800$

H $\quad 4.46431300 \quad 0.81777700 \quad-2.27754700$

H $\quad 4.68709800 \quad 2.55413700 \quad-1.96368100$

H $\quad 5.14252000 \quad 1.36917100 \quad-0.72659900$

H $\quad 1.89957000 \quad 2.89637400 \quad 0.57803300$

H $\quad 3.63590400 \quad 2.70337800 \quad 0.92752200$

H $\quad 3.11495900 \quad 3.80364000 \quad-0.36126100$

$\begin{array}{llll}\text { C } & 3.50383100 & -0.61524000 & 0.87956700\end{array}$

$\begin{array}{llll}\text { C } & 3.50186300 & -2.14956300 & 0.94393500\end{array}$

$\begin{array}{llll}\text { C } & 3.28765100 & -0.03753000 & 2.28670600\end{array}$

$\mathrm{H} \quad 4.49266300 \quad-0.28959100 \quad 0.50892300$

H $\quad 3.73959000 \quad-2.61400900 \quad-0.02473300$

Н $\quad 4.25802200 \quad-2.49594200 \quad 1.67218600$

H $\quad 2.52400100 \quad-2.53330200 \quad 1.27131000$

H $\quad 3.26917900 \quad 1.06156700 \quad 2.29942500$

H $\quad 2.32772800 \quad-0.38156000 \quad 2.70071800$

H $\quad 4.09212600 \quad-0.37090700 \quad 2.96762100$

$\begin{array}{lrrr}\text { C } & -2.97778100 & 1.70085500 & -0.91120200\end{array}$

$\begin{array}{llll}\text { C } & -2.89589200 & 2.83297800 & 0.11980100\end{array}$

C $\quad-4.38997600 \quad 1.59499000 \quad-1.50125700$
$\mathrm{H} \quad-2.27499200 \quad 1.95143100 \quad-1.72436300$

$\begin{array}{llll}\mathrm{H} & -1.89954600 & 2.89636600 & 0.57798400\end{array}$

H $\quad-3.11494000 \quad 3.80362700 \quad-0.36130300$

H $\quad-3.63587700 \quad 2.70337500 \quad 0.92749300$

H $\quad-4.46431700 \quad 0.81775100 \quad-2.27755500$

$\mathrm{H} \quad-5.14251100 \quad 1.36915600 \quad-0.72660500$

$\mathrm{H} \quad-4.68710000 \quad 2.55411500 \quad-1.96370000$

$\begin{array}{llll}\text { C } & -3.50383500 & -0.61525000 & 0.87955000\end{array}$

$\begin{array}{llll}\text { C } & -3.28773400 & -0.03744900 & 2.28666000\end{array}$

$\begin{array}{llll}\text { C } & -3.50179700 & -2.14956900 & 0.94400600\end{array}$

H $\quad-4.49266900 \quad-0.28966900 \quad 0.50885400$

H $\quad-3.26934800 \quad 1.06164900 \quad 2.29931700$

H $\quad-4.09220100 \quad-0.37084700 \quad 2.96757400$

$\mathrm{H} \quad-2.32779800 \quad-0.38138300 \quad 2.70071400$

$\mathrm{H} \quad-3.73946000 \quad-2.61408200 \quad-0.02464400$

$\mathrm{H} \quad-2.52393100 \quad-2.53324200 \quad 1.27144100$

$\mathrm{H} \quad-4.25797000 \quad-2.49594100 \quad 1.67224500$

$\begin{array}{llll}\mathrm{H} & 0.00000400 & 1.31738400 & -0.83379200\end{array}$

\section{[Mn]-1e}

P $\quad-1.77759000 \quad 0.08268900 \quad-0.48716500$

$\begin{array}{llll}\text { C } & -0.66999600 & -0.04795000 & -2.00720700\end{array}$

$\begin{array}{llll}\text { C } & 0.66459500 & -0.65734600 & -1.52979000\end{array}$

H $\quad-0.51128600 \quad 0.96544600 \quad-2.40677100$

H $\quad-1.14009500 \quad-0.63887200 \quad-2.80980000$

H $\quad 0.57563300 \quad-1.75431700 \quad-1.48113900$

H $\quad 1.47010900 \quad-0.43021300 \quad-2.24865900$

$\mathrm{N} \quad 0.96860300 \quad-0.12392400 \quad-0.18918100$

C $\quad 1.93047500 \quad 0.98387100 \quad-0.24373800$

H $\quad 1.93127400 \quad 1.49623900 \quad 0.72888400$

H $\quad 1.66252800 \quad 1.72975600 \quad-1.01443700$

$\begin{array}{llll}M n & -0.29318500 & -0.45533600 & 1.13657900\end{array}$

$\begin{array}{llll}\text { C } & -0.61488200 & -2.22838200 & 0.83190500\end{array}$

$\begin{array}{llll}\text { O } & -0.81370600 & -3.36143400 & 0.77045500\end{array}$

$\begin{array}{llll}\text { C } & -0.14478300 & 1.04456400 & 2.16484700\end{array}$

$\begin{array}{llll}\text { O } & -0.16514300 & 1.90420300 & 2.93354700\end{array}$

C $\quad-3.17402100 \quad-1.11745700 \quad-0.89080600$

C $\quad-4.05582100 \quad-0.67349200 \quad-2.06704200$

$\begin{array}{llll}\text { C } & -4.02111900 & -1.55007600 & 0.31446200\end{array}$

$\mathrm{H} \quad-2.59671000 \quad-2.00190500 \quad-1.21461400$

H $\quad-3.47198200 \quad-0.37933000 \quad-2.95338600$

Н $\quad-4.71540800 \quad-1.50306100 \quad-2.37099400$

H $\quad-4.70676600 \quad 0.17210400 \quad-1.79352600$

$\begin{array}{llll}\mathrm{H} & -3.40237700 & -1.88502200 & 1.15772900\end{array}$

H $\quad-4.67094300 \quad-0.73910800 \quad 0.67633300$ 


$$
\begin{array}{cccc}
\mathrm{H} & -4.67604200 & -2.38717900 & 0.02096200 \\
\mathrm{C} & -2.52073500 & 1.80785600 & -0.58041500 \\
\mathrm{C} & -1.44610100 & 2.90360700 & -0.54041400 \\
\mathrm{C} & -3.56840400 & 2.04144900 & 0.51707300 \\
\mathrm{H} & -3.01832200 & 1.85047100 & -1.56526300 \\
\mathrm{H} & -0.68712300 & 2.79234200 & -1.32873200 \\
\mathrm{H} & -1.92203100 & 3.88762500 & -0.68370200 \\
\mathrm{H} & -0.92665600 & 2.92706000 & 0.42851000 \\
\mathrm{H} & -4.42382100 & 1.35632700 & 0.43735900 \\
\mathrm{H} & -3.12986200 & 1.92146900 & 1.52013500 \\
\mathrm{H} & -3.96166700 & 3.06888200 & 0.44562000 \\
\mathrm{C} & 3.31958500 & 0.52511700 & -0.59204700 \\
\mathrm{C} & 5.29430900 & -0.44726300 & -0.42176600 \\
\mathrm{H} & 6.11597500 & -0.99587400 & 0.03323400 \\
\mathrm{~N} & 3.90040400 & 0.70523800 & -1.76500600 \\
\mathrm{~N} & 4.12769500 & -0.18283200 & 0.26370500 \\
\mathrm{C} & 5.13012400 & 0.10910900 & -1.67092400 \\
\mathrm{H} & 5.83490400 & 0.10581700 & -2.50188900 \\
\mathrm{C} & 3.83182500 & -0.54767100 & 1.63823100 \\
\mathrm{H} & 4.45807200 & -1.40131200 & 1.93143200 \\
\mathrm{H} & 4.03048500 & 0.28777700 & 2.33001500 \\
\mathrm{H} & 2.77618000 & -0.83876000 & 1.72338000 \\
\mathrm{H} & -0.14988300 & -1.06721900 & 2.55493400 \\
\mathrm{H} & -1.62713500 & -0.40320800 & 1.94453600
\end{array}
$$

\section{iso-I}

P $\quad-1.79362300 \quad 0.58521500 \quad 0.18486000$

$\begin{array}{llll}\text { C } & -0.46024900 & 1.78565200 & 0.74809600\end{array}$

C $\quad 0.86812200 \quad 1.05258700 \quad 0.92691700$

H $\quad-0.72868200 \quad 2.32921100 \quad 1.66724400$

$\mathrm{H} \quad-0.35702700 \quad 2.53912100 \quad-0.05032700$

H $\quad 1.68017900 \quad 1.77234800 \quad 1.12955200$

H $\quad 0.81221300 \quad 0.35399000 \quad 1.77606900$

$\begin{array}{llll}\mathrm{N} & 1.17036200 & 0.25774800 & -0.28468700\end{array}$

C $\quad 2.38206800 \quad-0.56318300 \quad-0.17338700$

H $\quad 2.38367600 \quad-1.29031800 \quad-1.00779500$

H $\quad 2.31948200 \quad-1.16267200 \quad 0.74871000$

$\begin{array}{llll}\mathrm{Mn} & -0.53238600 & -0.89403300 & -0.89546000\end{array}$

$\begin{array}{llll}\text { C } & -0.44377400 & -2.10942200 & 0.42835100\end{array}$

$\begin{array}{llll}\mathrm{O} & -0.42662700 & -2.93752200 & 1.25004500\end{array}$

C $\quad-1.83808800-1.70915300 \quad-1.78160200$

O $\quad-2.68414700 \quad-2.20151600 \quad-2.40591300$

C $\quad-2.90128300 \quad 1.73278000 \quad-0.82561100$

$\begin{array}{llll}\text { C } & -3.33370000 & 3.01686000 & -0.10412000\end{array}$

C $\quad-4.10965300 \quad 1.02881500 \quad-1.45905200$

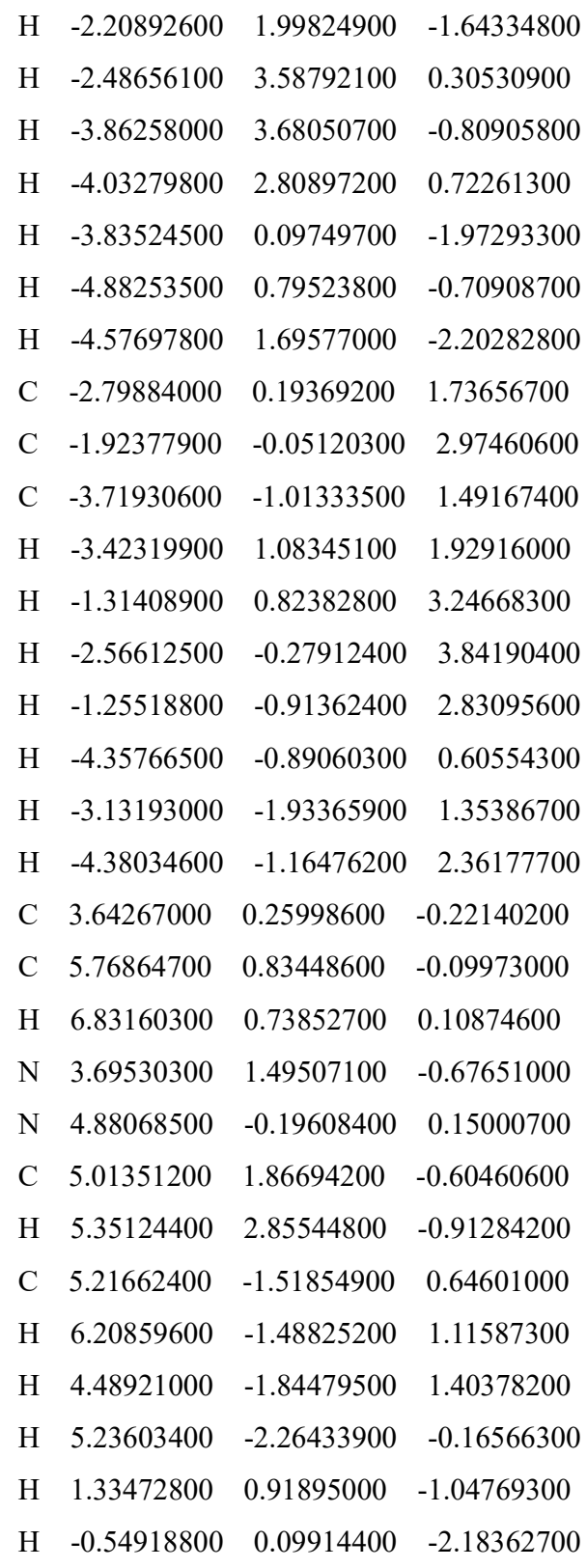

iso-II

$\begin{array}{llll}\mathrm{P} & 1.77820800 & -0.65335600 & 0.16502700\end{array}$

$\begin{array}{llll}\text { C } & 0.42783500 & -1.84903300 & 0.69372100\end{array}$

$\begin{array}{llll}\text { C } & -0.86554900 & -1.07268100 & 0.92716400\end{array}$

$\mathrm{H} \quad 0.68737500 \quad-2.44819500 \quad 1.58045900$

H $\quad 0.28175100 \quad-2.56182300 \quad-0.13649700$

H $\quad-1.70695900 \quad-1.76771300 \quad 1.08915400$

$\mathrm{H} \quad-0.77597600 \quad-0.43554900 \quad 1.82020900$

$\begin{array}{llll}\mathrm{N} & -1.15108400 & -0.17443000 & -0.22420000\end{array}$

$\begin{array}{llll}\text { C } & -2.38891500 & 0.60635300 & -0.03041200\end{array}$

$\mathrm{H} \quad-2.38074500 \quad 1.41642800 \quad-0.77614500$

$\mathrm{H} \quad-2.34638300 \quad 1.09451300 \quad 0.95647800$

$\begin{array}{llll}\text { Mn } & 0.53684200 & 1.01406600 & -0.82232700\end{array}$

C $\quad 0.58799800 \quad 2.16726700 \quad 0.49167500$ 


$$
\begin{aligned}
& \begin{array}{llll}
\mathrm{O} & 0.54353700 & 2.94883000 & 1.35761800
\end{array} \\
& \text { C } \quad 1.80544100 \quad 1.88861600 \quad-1.70274800 \\
& \begin{array}{lllll}
\mathrm{O} & 2.60075900 & 2.46876900 & -2.31874100
\end{array} \\
& \text { C } \quad 2.81990100 \quad-1.77341500 \quad-0.95082800 \\
& \text { C } 3.25419700 \quad-3.10558200 \quad-0.32529100 \\
& \text { C } 4.01157900 \quad-1.05205700 \quad-1.59773200 \\
& \mathrm{H} \quad 2.09413700 \quad-1.99396300 \quad-1.75672200 \\
& \text { H } \quad 2.41089100 \quad-3.68004800 \quad 0.08726300 \\
& \text { H } \quad 3.74117000 \quad-3.73933600 \quad-1.08579200 \\
& \text { H } 3.98838800 \quad-2.95509900 \quad 0.48279100 \\
& \text { H } \quad 3.73692300 \quad-0.07481200 \quad-2.02006300 \\
& \mathrm{H} \quad 4.82642400 \quad-0.89260300 \quad-0.87386700 \\
& \mathrm{H} \quad 4.42359200 \quad-1.66899900 \quad-2.41364600 \\
& \begin{array}{llll}
\text { C } & 2.82961000 & -0.37902300 & 1.70822400
\end{array} \\
& \begin{array}{llll}
\text { C } & 1.97636300 & -0.12659500 & 2.96020000
\end{array} \\
& \begin{array}{llll}
\text { C } & 3.81269600 & 0.78360100 & 1.49722400
\end{array} \\
& \text { H } \quad 3.40272100 \quad-1.30984600 \quad 1.86292600 \\
& \text { H } \quad 1.31420200 \quad-0.97106400 \quad 3.20410900 \\
& \begin{array}{llll}
\mathrm{H} & 2.63670000 & 0.02714000 & 3.83006600
\end{array} \\
& \text { H } \quad 1.36343400 \quad 0.78156500 \quad 2.85265000 \\
& \text { H } \quad 4.45187300 \quad 0.64968400 \quad 0.61348900 \\
& \mathrm{H} \quad 3.27425000 \quad 1.73573900 \quad 1.37770500 \\
& \mathrm{H} \quad 4.47307100 \quad 0.87836700 \quad 2.37562100 \\
& \text { C } \quad-3.61618700 \quad-0.25101400 \quad-0.18510100 \\
& \begin{array}{llll}
\text { C } & -5.72526700 & -0.89860600 & -0.20954300
\end{array} \\
& \mathrm{H} \quad-6.79847500 \quad-0.85313800 \quad-0.04023900 \\
& \mathrm{~N} \quad-3.60929800 \quad-1.44125900 \quad-0.75247200 \\
& \begin{array}{llll}
\mathrm{N} & -4.88199400 & 0.13061800 & 0.17087000
\end{array} \\
& \begin{array}{llll}
\text { C } & -4.91684600 & -1.85767700 & -0.77197900
\end{array} \\
& \text { H } \quad-5.20881500 \quad-2.82333700 \quad-1.18245400 \\
& \begin{array}{llll}
\text { C } & -5.28115400 & 1.39820800 & 0.75524500
\end{array} \\
& \mathrm{H} \quad-6.27870400 \quad 1.29386300 \quad 1.20245100 \\
& \mathrm{H} \quad-4.57923300 \quad 1.69584600 \quad 1.54772900 \\
& \mathrm{H} \quad-5.31553700 \quad 2.20003300 \quad-0.00042300 \\
& \mathrm{H} \quad-1.35510000 \quad-0.78797500 \quad-1.02472600 \\
& \mathrm{H} \quad-0.50307000 \quad 1.82353500 \quad-1.71907800
\end{aligned}
$$

\section{iso-III}

$\mathrm{P} \quad-1.73981800 \quad-0.66339200 \quad-0.14285900$

$$
\begin{array}{llll}
\mathrm{C} & -0.37207700 & -1.93549700 & -0.38969300 \\
\mathrm{C} & 0.88577800 & -1.24167100 & -0.91224400 \\
\mathrm{H} & -0.66437700 & -2.75757600 & -1.06275900 \\
\mathrm{H} & -0.15584100 & -2.39011100 & 0.59209600 \\
\mathrm{H} & 1.74394300 & -1.93360500 & -0.87790700 \\
\mathrm{H} & 0.75065100 & -0.92990100 & -1.96073400
\end{array}
$$

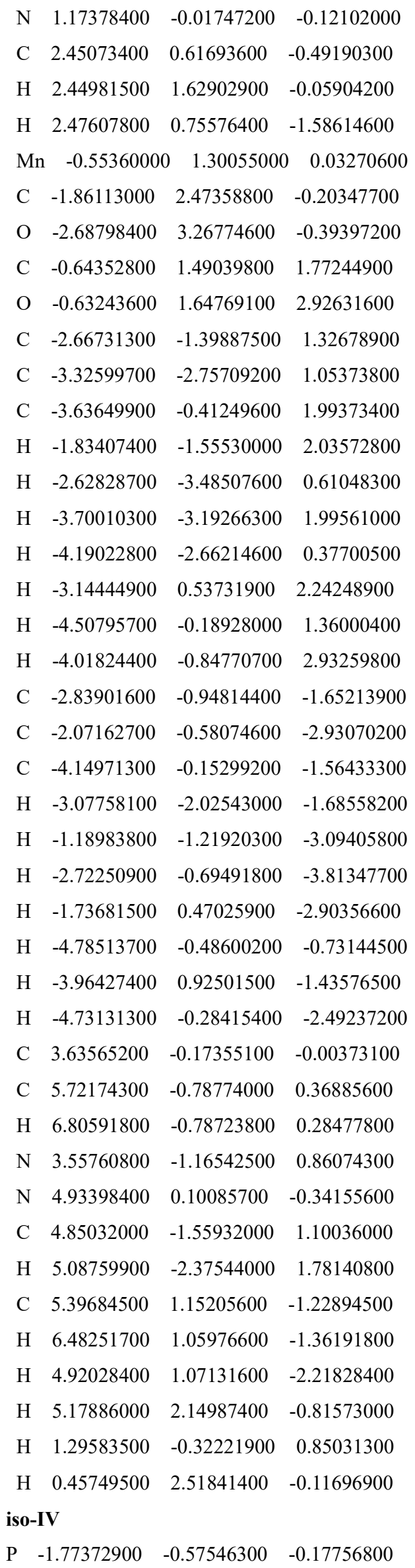




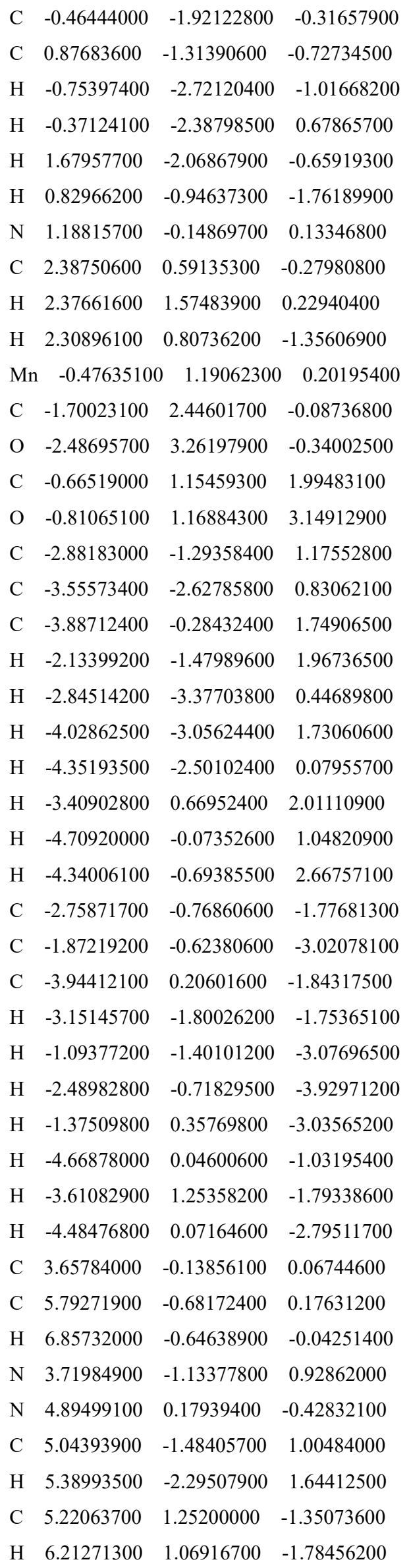

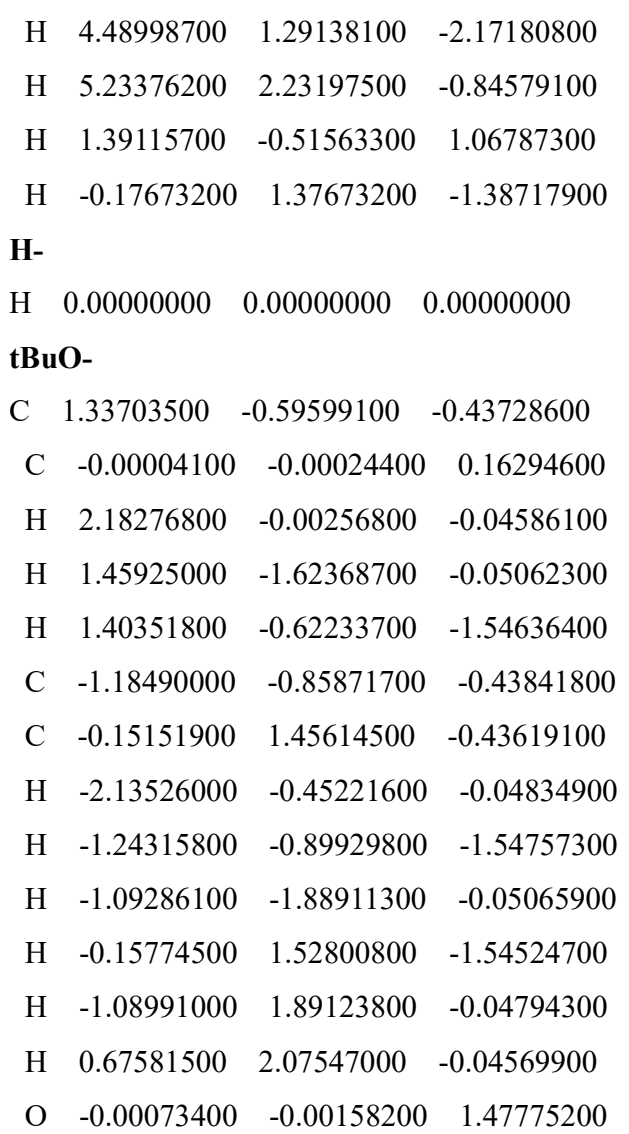




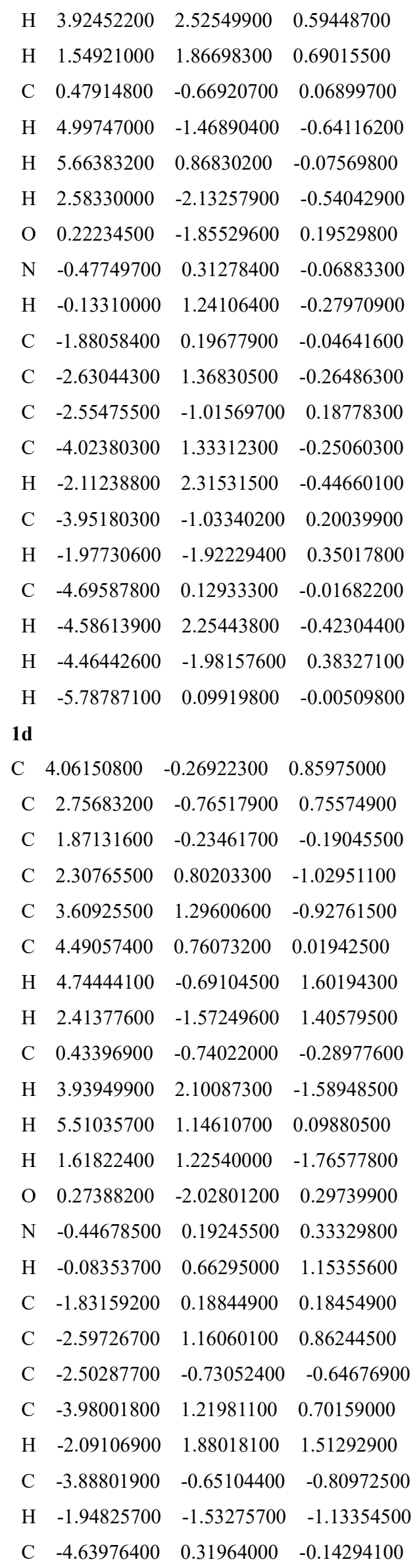

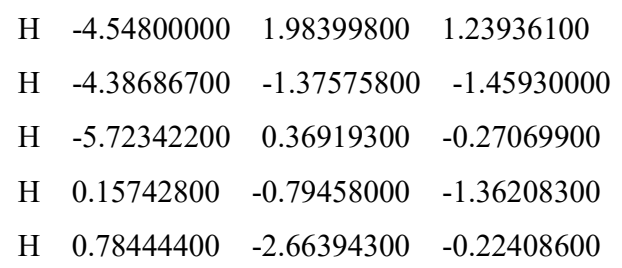




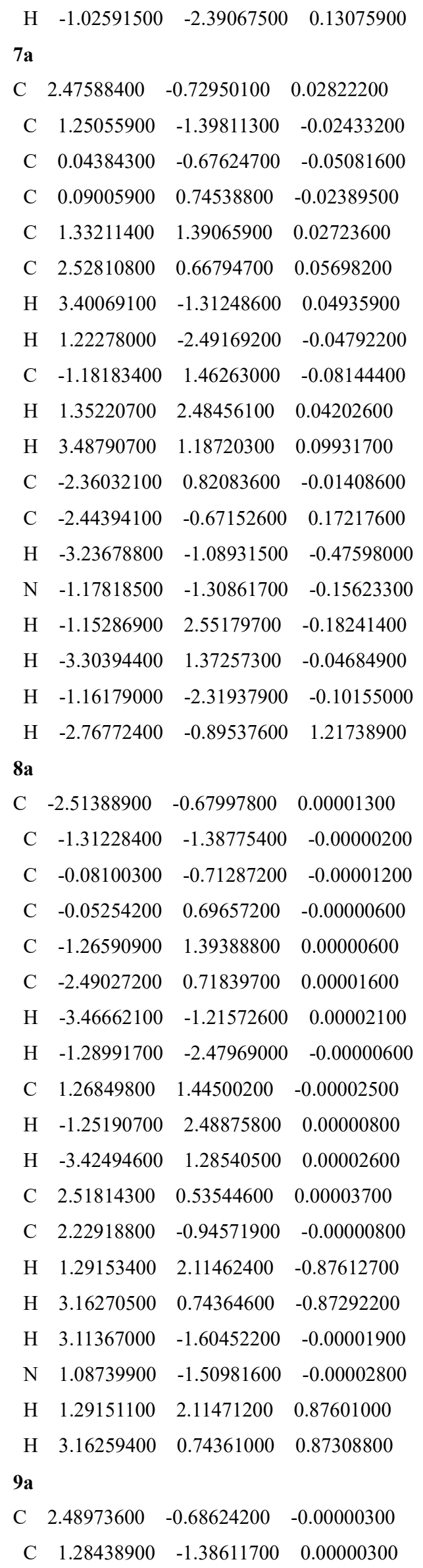

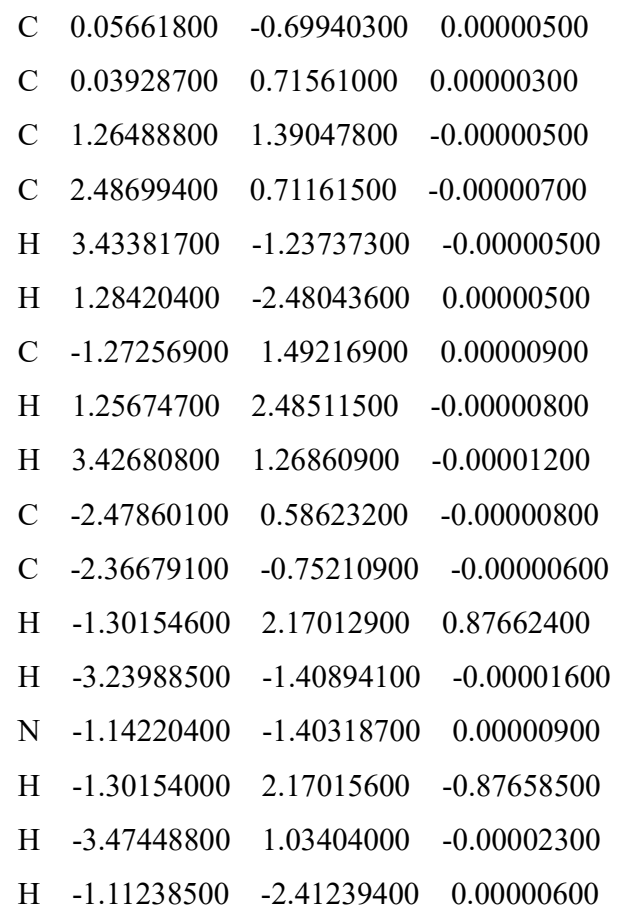




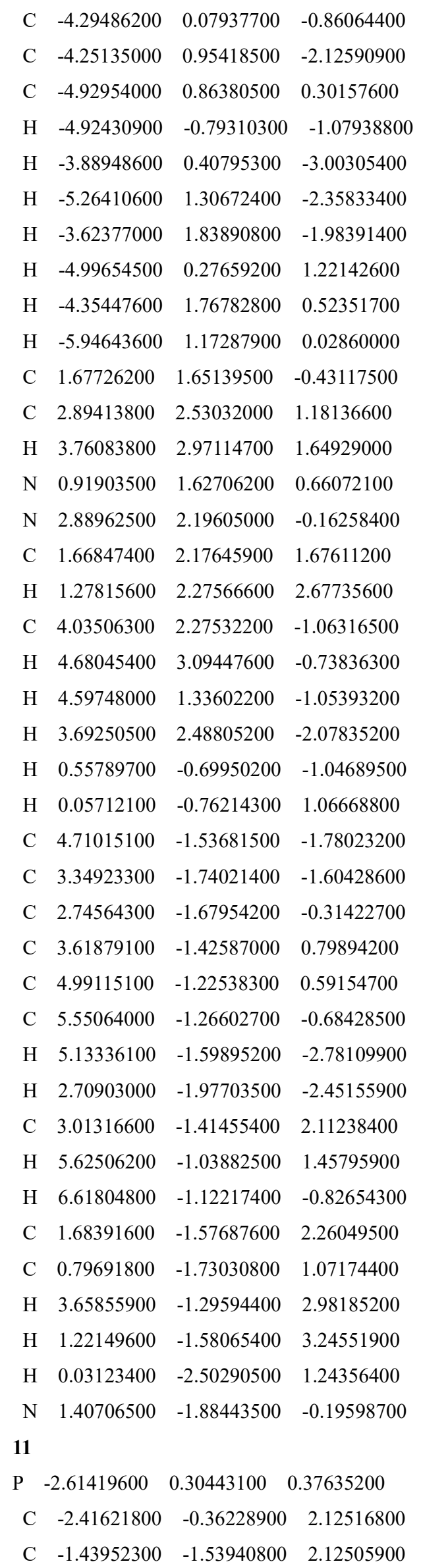

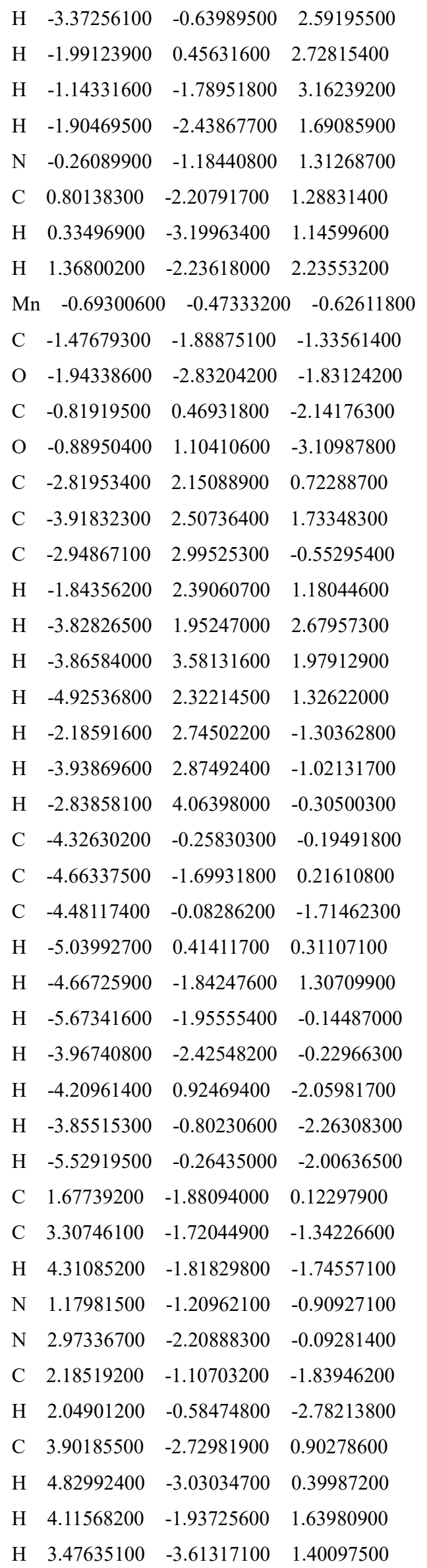




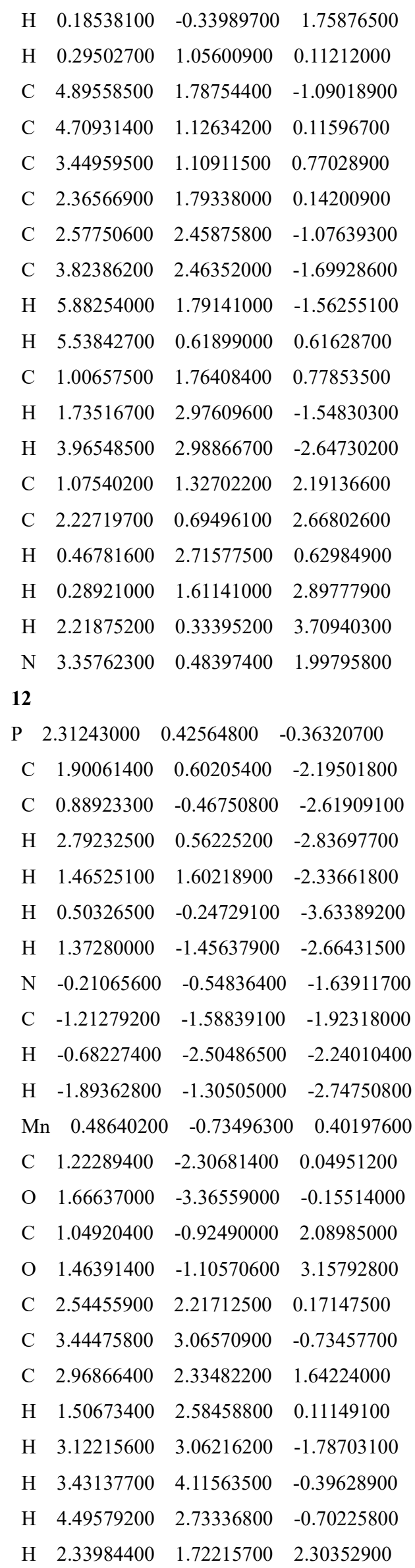

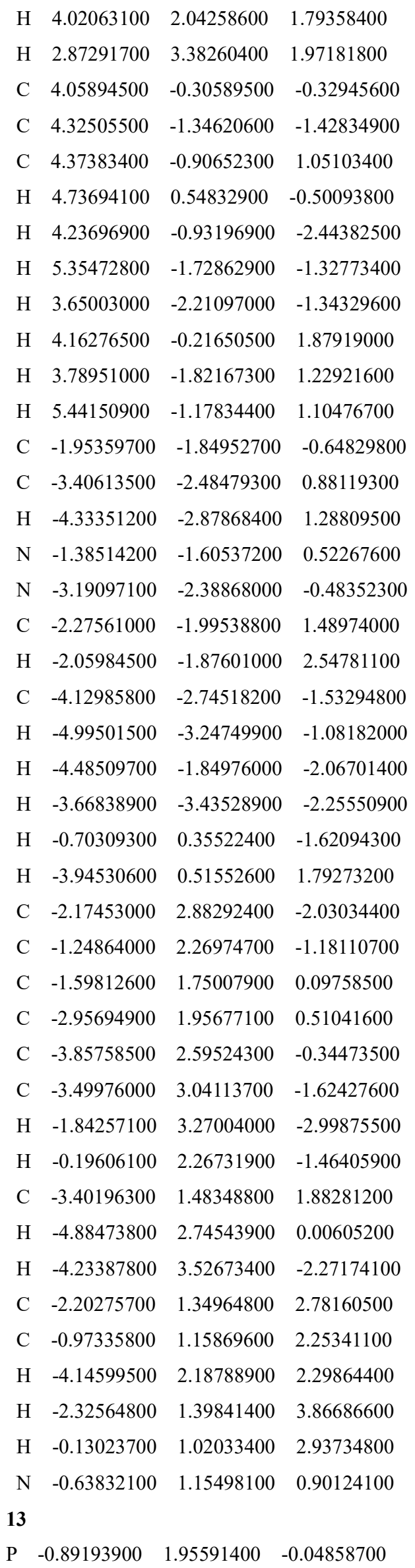




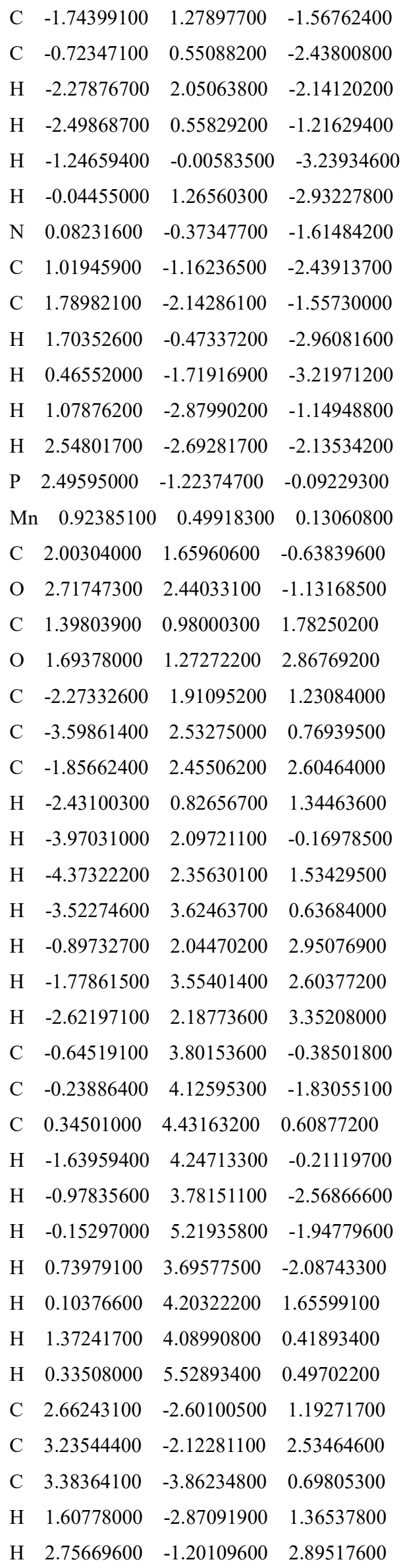

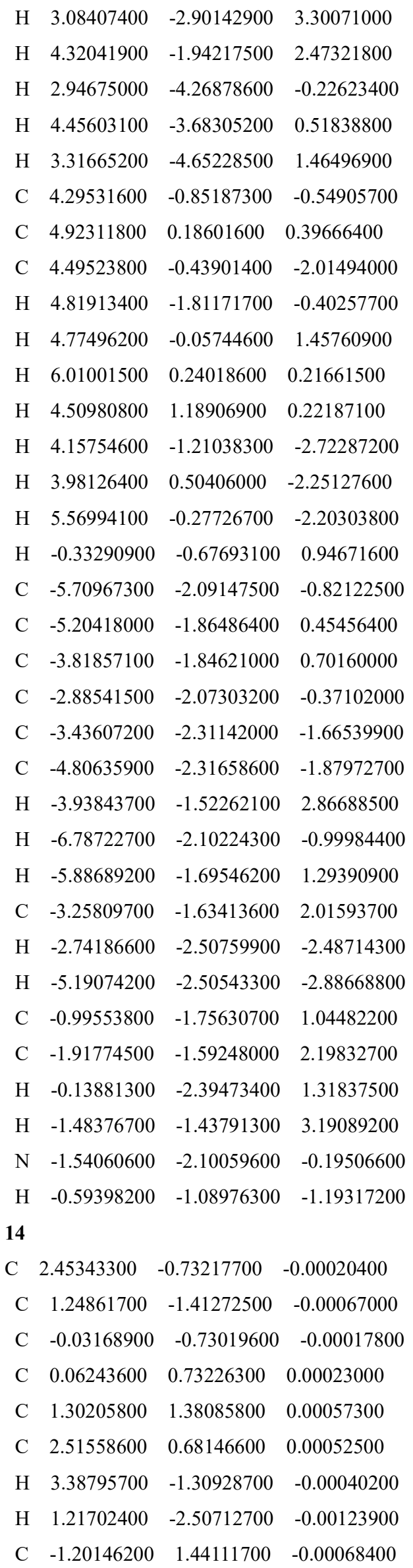




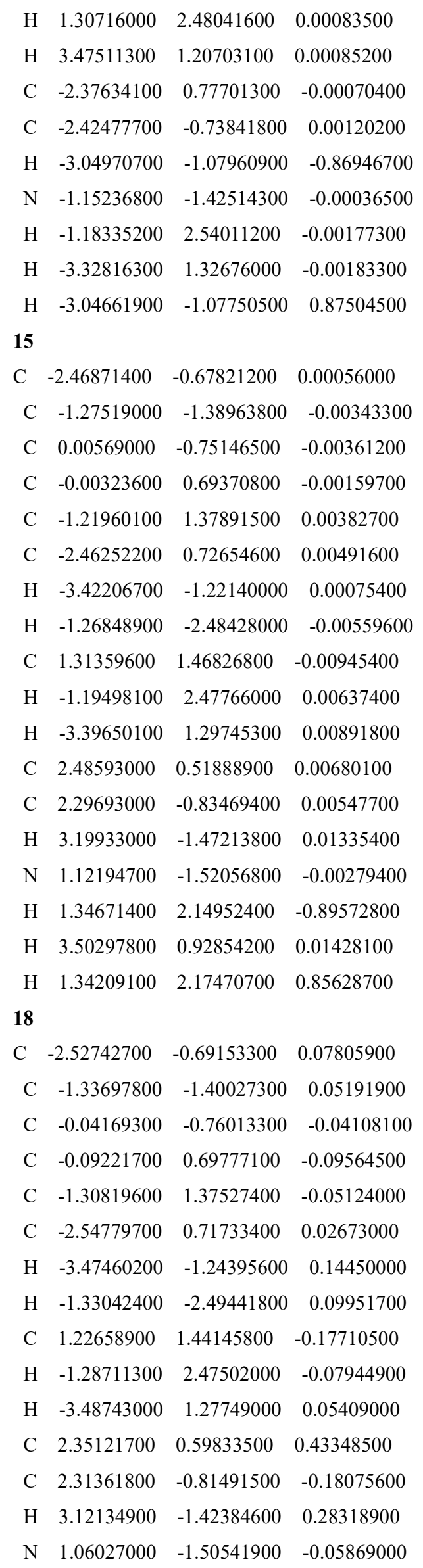

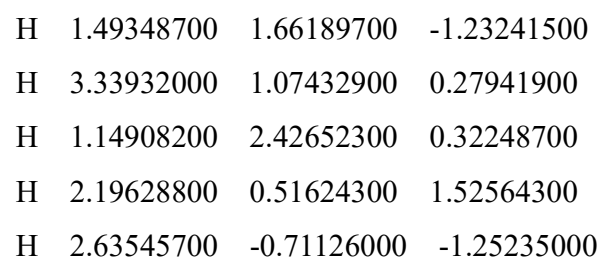




$$
\begin{aligned}
& \begin{array}{llll}
\text { C } & -3.13837500 & -0.88261000 & -0.00339500
\end{array} \\
& \begin{array}{llll}
\text { C } & -2.93886600 & 0.51679300 & 0.09049700
\end{array} \\
& \begin{array}{llll}
\text { C } & -3.93487800 & 1.37799000 & -0.38195800
\end{array} \\
& \begin{array}{llll}
\text { C } & -5.11903100 & 0.88149900 & -0.93976500
\end{array} \\
& \mathrm{H} \quad-6.23876100 \quad-0.89905600 \quad-1.46096000 \\
& \begin{array}{lllll}
\mathrm{H} & -4.45612500 & -2.45807600 & -0.60980100
\end{array} \\
& \begin{array}{llll}
\text { C } & -1.66075100 & 0.99798300 & 0.73317000
\end{array} \\
& \begin{array}{llll}
\mathrm{H} & -3.77769500 & 2.45977800 & -0.31291000
\end{array} \\
& \mathrm{H} \quad-5.88454800 \quad 1.57278300 \quad-1.30419300 \\
& \begin{array}{llll}
\text { C } & -0.49704400 & 0.03043800 & 0.46861900
\end{array} \\
& \begin{array}{llll}
\text { C } & -0.98066200 & -1.38771300 & 0.65348300
\end{array} \\
& \mathrm{H} \quad-0.20974100 \quad-2.09930300 \quad 0.97862000 \\
& \mathrm{~N} \quad-2.18029300 \quad-1.80735900 \quad 0.45065400 \\
& \begin{array}{llll}
\mathrm{H} & -1.81050900 & 1.06453400 & 1.83149000
\end{array} \\
& \begin{array}{llll}
\text { C } & 4.88098000 & -1.35202300 & -0.72497100
\end{array} \\
& \begin{array}{llll}
\text { C } & 3.76775600 & -1.54459200 & 0.07868200
\end{array} \\
& \begin{array}{llll}
\text { C } & 2.79023100 & -0.51205200 & 0.31639500
\end{array} \\
& \begin{array}{llll}
\text { C } & 3.05619700 & 0.74754500 & -0.35335300
\end{array} \\
& \begin{array}{llll}
\text { C } & 4.19371800 & 0.91234700 & -1.14299600
\end{array} \\
& \text { C } \quad 5.12371500 \quad-0.11612500 \quad-1.35485100 \\
& \mathrm{H} \quad 5.58665300 \quad-2.18049000 \quad-0.86617100 \\
& \begin{array}{llll}
\mathrm{H} & 3.58906000 & -2.50477700 & 0.57264500
\end{array} \\
& \begin{array}{llll}
\text { C } & 2.05814700 & 1.86721500 & -0.15519000
\end{array} \\
& \mathrm{H} \quad 4.35678300 \quad 1.89028700 \quad-1.61758900 \\
& \begin{array}{lllll}
\mathrm{H} & 6.00533800 & 0.03947500 & -1.98343300
\end{array} \\
& \text { C } \quad 1.34947900 \quad 1.70139300 \quad 1.19243000 \\
& \begin{array}{llll}
\text { C } & 0.78898900 & 0.26850700 & 1.34705000
\end{array} \\
& \mathrm{H} \quad 2.55833900 \quad 2.85166700 \quad-0.21553100 \\
& \begin{array}{llll}
\mathrm{H} & 2.08519500 & 1.85278000 & 2.00199300
\end{array} \\
& \begin{array}{llll}
\mathrm{H} & 0.41921300 & 0.19029400 & 2.39629500
\end{array} \\
& \begin{array}{llll}
\mathrm{N} & 1.74777800 & -0.77057900 & 1.11768500
\end{array} \\
& \mathrm{H} \quad 0.56509500 \quad 2.46627900 \quad 1.33396600 \\
& \mathrm{H} \quad 1.30003600 \quad 1.87325600 \quad-0.96607000 \\
& \mathrm{H} \quad-1.42553600 \quad 2.01761100 \quad 0.39229700 \\
& \mathrm{H} \quad-0.17691800 \quad 0.11839000 \quad-0.58831300
\end{aligned}
$$$$
23
$$$$
\text { P } \quad-0.01073000 \quad 2.45868300 \quad 0.00305300
$$$$
\begin{array}{llll}
\text { C } & 1.85557500 & 2.49790600 & -0.02358700
\end{array}
$$$$
\text { C } \quad 2.40338300 \quad 1.21722800 \quad 0.58895400
$$$$
\text { H } \quad 2.27069100 \quad 3.38082300 \quad 0.48518500
$$$$
\text { H } 2.16646800 \quad 2.55857500 \quad-1.08002300
$$$$
\text { H } \quad 3.50273100 \quad 1.19442100 \quad 0.48622100
$$$$
\mathrm{H} \quad 2.16478800 \quad 1.16822600 \quad 1.66285900
$$$$
\text { N } \quad 1.79479300 \quad 0.03412200 \quad-0.05738300
$$

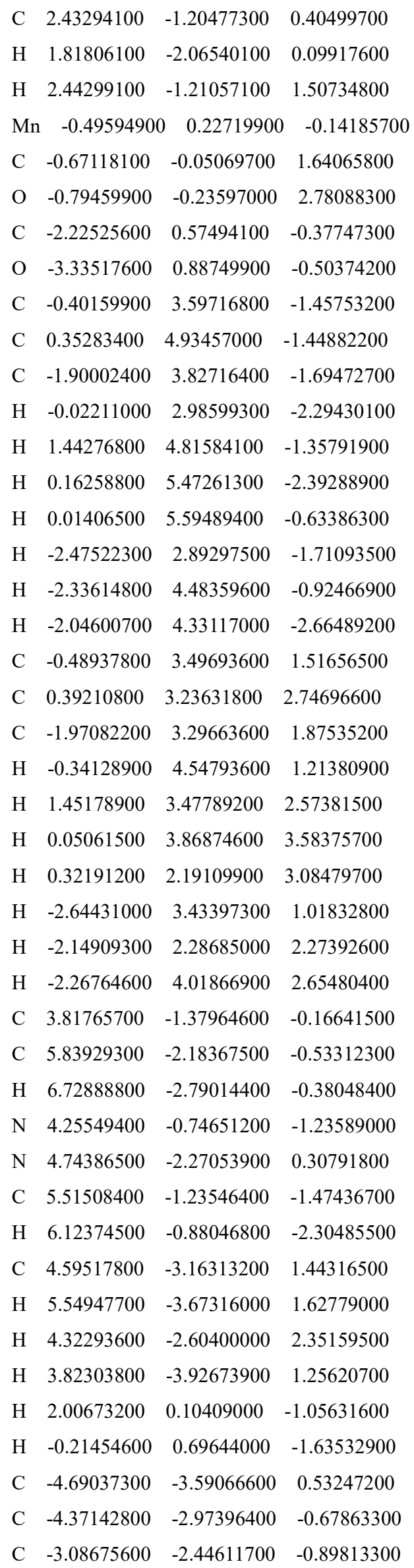




\begin{tabular}{|c|c|c|c|c|c|c|c|}
\hline $\mathrm{C}$ & -2.10956100 & -2.54487200 & 0.12843900 & $\mathrm{C}$ & -0.21306700 & 3.53072300 & 2.48350400 \\
\hline $\mathrm{C}$ & -2.45265400 & -3.17834200 & 1.33025800 & $\mathrm{C}$ & -2.62186300 & 3.31442500 & 1.75958900 \\
\hline $\mathrm{C}$ & -3.73078100 & -3.70177600 & 1.54409800 & $\mathrm{H}$ & -1.12708600 & 4.57453400 & 0.84446800 \\
\hline $\mathrm{H}$ & -5.69632700 & -3.99238900 & 0.68185100 & $\mathrm{H}$ & 0.81191500 & 3.82058600 & 2.20463500 \\
\hline $\mathrm{H}$ & -5.12327200 & -2.89501100 & -1.46990400 & $\mathrm{H}$ & -0.55003800 & 4.24644300 & 3.25206200 \\
\hline $\mathrm{C}$ & -0.76519800 & -1.99101700 & -0.11859900 & $\mathrm{H}$ & -0.17419400 & 2.53618300 & 2.95229100 \\
\hline $\mathrm{H}$ & -1.69714500 & -3.24756600 & 2.11859800 & $\mathrm{H}$ & -3.35568100 & 3.42677900 & 0.94837900 \\
\hline $\mathrm{H}$ & -3.97598000 & -4.18825100 & 2.49104800 & $\mathrm{H}$ & -2.73985700 & 2.30296400 & 2.17696200 \\
\hline $\mathrm{C}$ & -0.41510500 & -1.50422800 & -1.39312900 & $\mathrm{H}$ & -2.89124300 & 4.03659900 & 2.54855100 \\
\hline $\mathrm{C}$ & -1.38770300 & -1.71419000 & -2.53826500 & $\mathrm{C}$ & 3.94740300 & -0.48779400 & -0.25791900 \\
\hline $\mathrm{H}$ & 0.00418900 & -2.37433600 & 0.55430400 & $\mathrm{C}$ & 6.01088700 & -1.02774700 & -0.81975000 \\
\hline $\mathrm{H}$ & 0.62608700 & -1.60046500 & -1.71777800 & $\mathrm{H}$ & 7.01471200 & -1.43180500 & 23400 \\
\hline $\mathrm{H}$ & -1.08923200 & -2.65098500 & -3.06773500 & $\mathrm{~N}$ & 4.09108200 & -0.06926000 & -1.49970000 \\
\hline $\mathrm{N}$ & -2.75947000 & -1.78596100 & -2.06923500 & $\mathrm{~N}$ & 5.08874400 & -1.08007600 & 0.21067400 \\
\hline $\mathrm{H}$ & -1.30828600 & -0.89746400 & -3.27226600 & $\mathrm{C}$ & 5.37386700 & -0.39699800 & -1.86197100 \\
\hline $\mathrm{H}$ & -3.47024800 & -1.79592600 & -2.79034600 & $\mathrm{H}$ & 5.77363200 & -0.16077300 & -2.84709100 \\
\hline 24 & & & & $\mathrm{C}$ & 5.27684300 & -1.69802300 & 1.51111100 \\
\hline $\mathrm{P}$ & $-0.67394100 \quad 2$ & 2.36598500 & -0.07524600 & $\mathrm{H}$ & 6.34284000 & -1.91579600 & 1.65723800 \\
\hline $\mathrm{C}$ & 1.07363000 & 2.90143500 & -0.48911500 & $\mathrm{H}$ & 4.94997500 & -1.02166400 & 2.31518700 \\
\hline $\mathrm{C}$ & 2.05249500 & 1.93136400 & 0.16100900 & $\mathrm{H}$ & 4.71144300 & -2.64027700 & 1.59465400 \\
\hline $\mathrm{H}$ & 1.29464500 & 3.93469200 & -0.17661500 & $\mathrm{H}$ & 1.86042800 & 0.39324900 & -1.14621800 \\
\hline $\mathrm{H}$ & 1.18592900 & 2.86977900 & -1.58645500 & $\mathrm{H}$ & -0.66640700 & -0.01045700 & -1.56314600 \\
\hline $\mathrm{H}$ & 3.08424800 & 2.14661500 & -0.16941400 & $\mathrm{C}$ & -3.23727600 & -4.77250200 & 0.63985600 \\
\hline $\mathrm{H}$ & 2.02026300 & 2.04324100 & 1.25485600 & $\mathrm{C}$ & -3.45287300 & -3.87530100 & -0.40893300 \\
\hline $\mathrm{N}$ & 1.70112700 & 0.51977800 & -0.13981000 & $\mathrm{C}$ & -2.46882600 & -2.94374800 & -0.76838300 \\
\hline $\mathrm{C}$ & 2.65737000 & -0.39350600 & 0.51287200 & $\mathrm{C}$ & -1.23688400 & -2.89467800 & -0.06447300 \\
\hline $\mathrm{H}$ & 2.20062000 & -1.39045700 & 0.59596900 & $\mathrm{C}$ & -1.03879800 & -3.82558900 & 0.96793200 \\
\hline $\mathrm{H}$ & 2.82331700 & -0.05338500 & 1.54738800 & $\mathrm{C}$ & -2.01924600 & -4.75412700 & 1.32637300 \\
\hline $\mathrm{Mn}$ & -0.48889000 & 0.10478700 & 0.23059000 & $\mathrm{H}$ & -4.01704700 & -5.48901200 & 0.91090500 \\
\hline $\mathrm{C}$ & -0.13619300 & -0.01185200 & 1.95853600 & $\mathrm{H}$ & -4.39770400 & -3.89363200 & -0.96198900 \\
\hline $\mathrm{O}$ & 0.14113800 & -0.14365000 & 3.08368400 & $\mathrm{C}$ & -0.22459600 & -1.86091200 & -0.39663200 \\
\hline $\mathrm{C}$ & -2.22658900 & -0.08754700 & 0.59063900 & $\mathrm{H}$ & -0.09182800 & -3.79980800 & 1.51634100 \\
\hline $\mathrm{O}$ & -3.33868600 & -0.15653700 & 0.91143900 & $\mathrm{H}$ & -1.83426100 & -5.45778700 & 2.14216200 \\
\hline $\mathrm{C}$ & -1.59639400 & 2.97133300 & -1.61809600 & $\mathrm{C}$ & -0.39320400 & -1.13945800 & -1.70471700 \\
\hline $\mathrm{C}$ & -1.64595600 & 4.49388500 & -1.79941600 & $\mathrm{C}$ & -1.50457900 & -1.69172200 & -2.60406800 \\
\hline $\mathrm{C}$ & -2.98262700 & 2.33480800 & -1.79413300 & $\mathrm{H}$ & 0.79046800 & -2.24952200 & -0.24233900 \\
\hline $\mathrm{H}$ & -0.95132200 & 2.55842700 & -2.41579700 & $\mathrm{H}$ & 0.54446700 & -1.07947100 & -2.28266100 \\
\hline $\mathrm{H}$ & -0.65574200 & 4.96666200 & -1.70127400 & $\mathrm{H}$ & -1.09027400 & -2.57869700 & -3.13248500 \\
\hline $\mathrm{H}$ & -2.03039600 & 4.74321900 & -2.80298100 & $\mathrm{~N}$ & -2.67193300 & -2.02218100 & -1.80858400 \\
\hline $\mathrm{H}$ & -2.32169000 & 4.96874800 & -1.07042900 & $\mathrm{H}$ & -1.79396200 & -0.95860800 & -3.37474600 \\
\hline $\mathrm{H}$ & -2.94294300 & 1.23748100 & -1.73954000 & $\mathrm{H}$ & -3.50320900 & -2.21047800 & -2.35894300 \\
\hline $\mathrm{H}$ & -3.69793900 & 2.67560700 & -1.03037300 & 25 & & & \\
\hline $\mathrm{H}$ & -3.39809600 & 2.61258900 & -2.77764700 & $\mathrm{P}$ & 2.37203200 & $34774300-$ & -0.33072700 \\
\hline $\mathrm{C}$ & -1.18175300 & 3.56710700 & 1.29205400 & $c$ & 1.89140600 & 0.86284100 & -2.07722400 \\
\hline
\end{tabular}




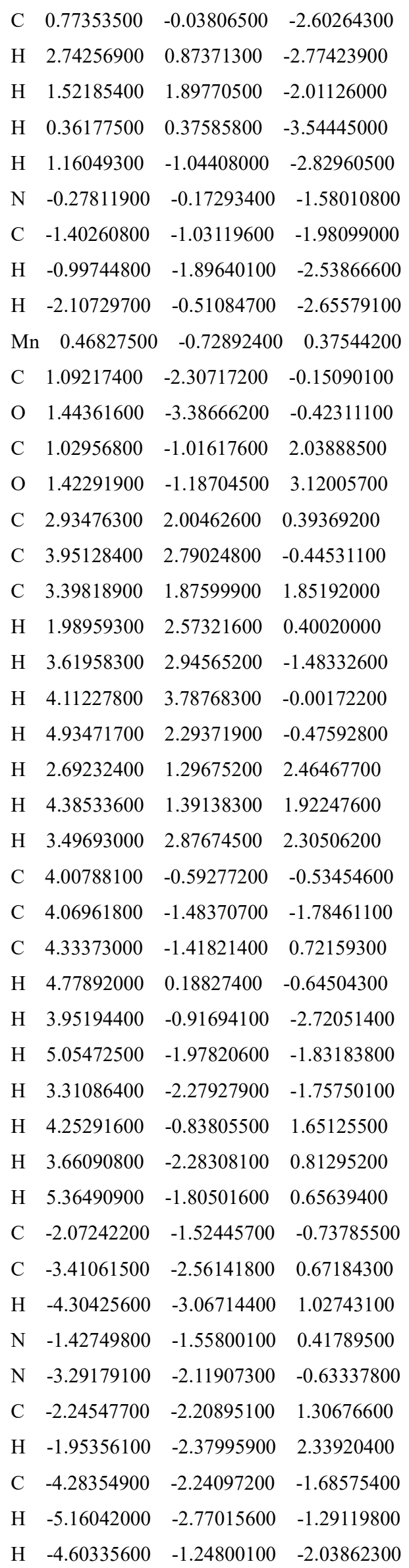

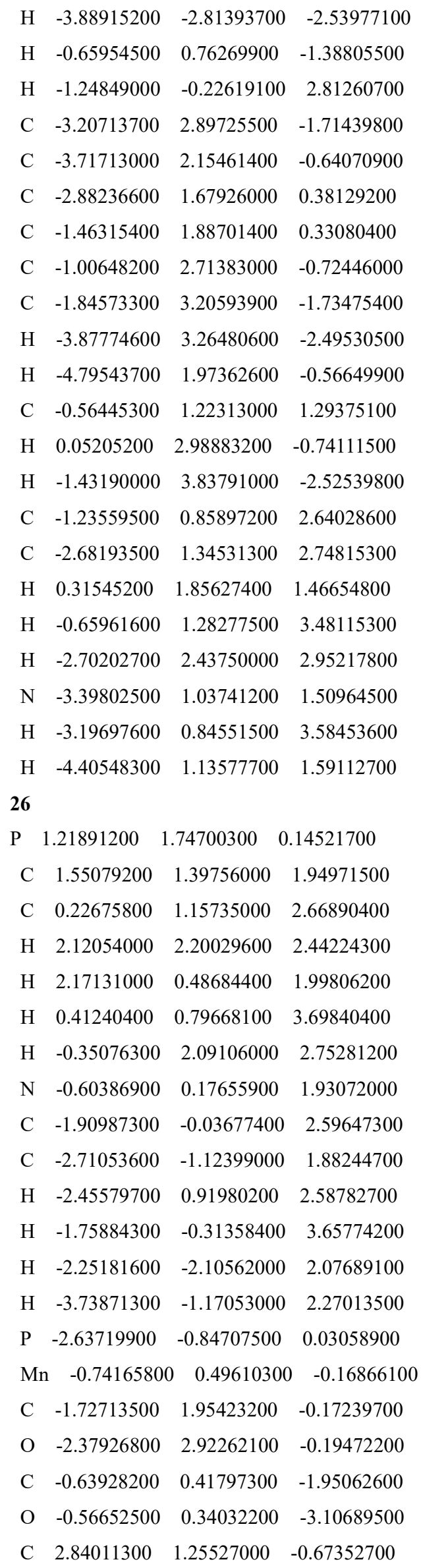




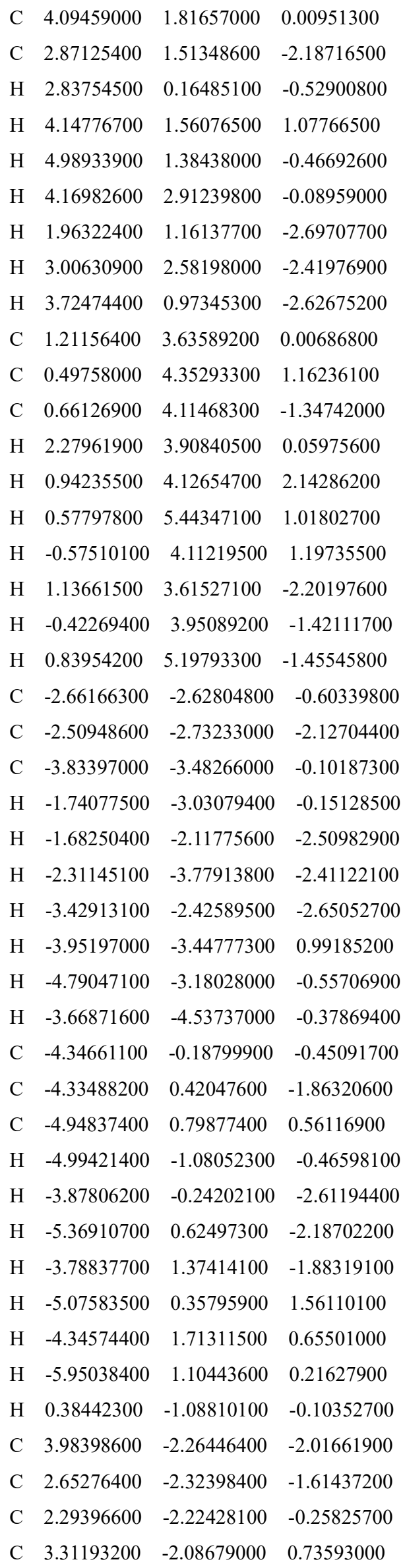

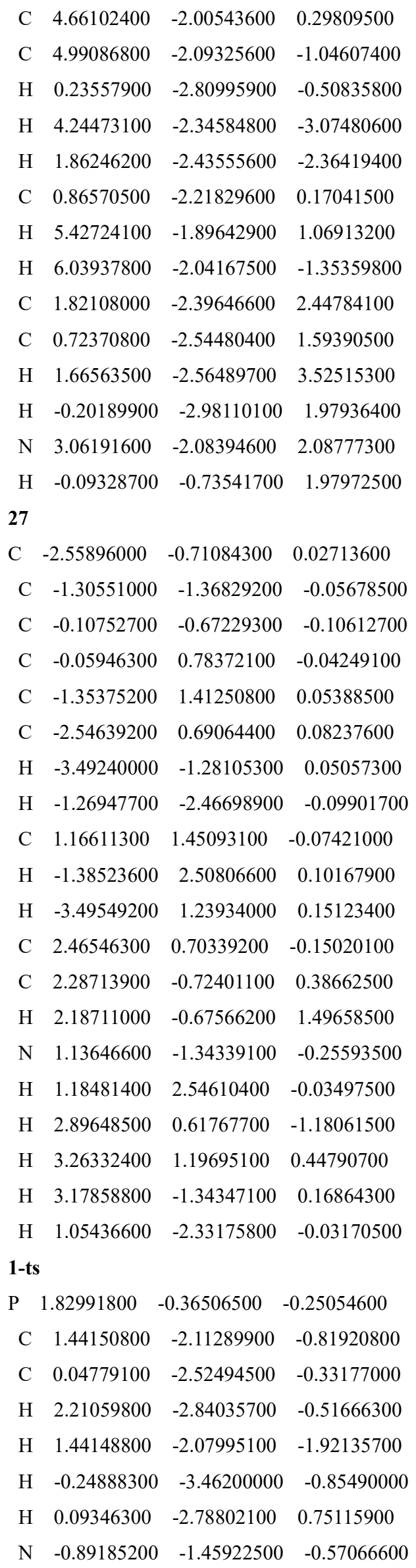




$$
\begin{aligned}
& \text { C } \quad-2.25057400 \quad-1.73525200 \quad-0.19573400 \\
& \text { H } \quad-2.32454700 \quad-2.15451800 \quad 0.84037700 \\
& \begin{array}{lllll}
\mathrm{H} & -2.74816300 & -2.48862900 & -0.85076500
\end{array} \\
& \begin{array}{llll}
\text { Mn } & -0.27708200 & 0.51756100 & -0.05265900
\end{array} \\
& \text { C } \quad-0.31612700 \quad 0.46070200 \quad 1.73754100 \\
& \begin{array}{lllll}
\mathrm{O} & -0.35627700 & 0.44167100 & 2.89853400
\end{array} \\
& \begin{array}{llll}
\text { C } & 0.13786300 & 2.25492900 & -0.08389300
\end{array} \\
& \begin{array}{llll}
\text { O } & 0.38670500 & 3.38877300 & -0.13226600
\end{array} \\
& \begin{array}{llll}
\text { C } & 2.92642300 & 0.22557900 & -1.67524800
\end{array} \\
& \begin{array}{llll}
\text { C } & 4.12271100 & -0.68098700 & -1.99465000
\end{array} \\
& \begin{array}{llll}
\text { C } & 3.36610100 & 1.69205400 & -1.56105200
\end{array} \\
& \begin{array}{lllll}
\mathrm{H} & 2.20853600 & 0.16417800 & -2.51270400
\end{array} \\
& \begin{array}{llll}
\mathrm{H} & 3.83695600 & -1.73488500 & -2.12996400
\end{array} \\
& \begin{array}{llll}
\mathrm{H} & 4.60698800 & -0.34941200 & -2.92887300
\end{array} \\
& \text { H } 4.89033500 \quad-0.63695600 \quad-1.20484600 \\
& \begin{array}{llll}
\mathrm{H} & 2.53472700 & 2.36438400 & -1.30907000
\end{array} \\
& \begin{array}{lllll}
\mathrm{H} & 4.15359000 & 1.82245600 & -0.80172300
\end{array} \\
& \begin{array}{llll}
\mathrm{H} & 3.79061300 & 2.02842000 & -2.52205300
\end{array} \\
& \begin{array}{llll}
\text { C } & 3.00766700 & -0.57985500 & 1.20895500
\end{array} \\
& \begin{array}{llll}
\text { C } & 2.53381400 & -1.64840100 & 2.20562400
\end{array} \\
& \begin{array}{llll}
\text { C } & 3.25070800 & 0.75419100 & 1.93296100
\end{array} \\
& \text { H } \quad 3.96153300 \quad-0.92350700 \quad 0.77241400 \\
& \begin{array}{llll}
\mathrm{H} & 2.42932800 & -2.64232600 & 1.74583500
\end{array} \\
& \text { H } \quad 3.27076300 \quad-1.74257400 \quad 3.02121400 \\
& \begin{array}{llll}
\mathrm{H} & 1.57159900 & -1.37787600 & 2.66553600
\end{array} \\
& \begin{array}{llll}
\mathrm{H} & 3.59000100 & 1.55137000 & 1.25705600
\end{array} \\
& \begin{array}{llll}
\mathrm{H} & 2.33574400 & 1.10623100 & 2.43219000
\end{array} \\
& \text { H } \quad 4.02377400 \quad 0.62418900 \quad 2.70921500 \\
& \begin{array}{lrrr}
\text { C } & -2.98865200 & -0.43282800 & -0.23640300
\end{array} \\
& \text { C } \quad-4.51187200 \quad 1.16736700 \quad-0.27086200 \\
& \mathrm{H} \quad-5.50082900 \quad 1.61605900 \quad-0.31224500 \\
& \begin{array}{llll}
\mathrm{N} & -2.32614600 & 0.71351500 & -0.17571500
\end{array} \\
& \text { N } \quad-4.32923300 \quad-0.20603100 \quad-0.30144800 \\
& \text { C } \quad-3.25791500 \quad 1.72137400 \quad-0.19672200 \\
& \begin{array}{llll}
\mathrm{H} & -2.97162200 & 2.76984500 & -0.16477100
\end{array} \\
& \begin{array}{lrrr}
\text { C } & -5.37977000 & -1.20730200 & -0.31710800
\end{array} \\
& \text { H } \quad-6.27384300 \quad-0.79090400 \quad-0.80085900 \\
& \mathrm{H} \quad-5.05272300 \quad-2.08617300 \quad-0.88979100 \\
& \mathrm{H} \quad-5.64600200 \quad-1.52944000 \quad 0.70293100 \\
& \mathrm{H} \quad-0.59366800 \quad-0.42916600 \quad-1.50832300 \\
& \mathrm{H} \quad-0.30233000 \quad 0.44279600 \quad-1.80428000
\end{aligned}
$$

\section{1-ts-quinoline}

P $\quad-2.45775500 \quad-0.82659000 \quad-0.28754500$

$\begin{array}{llll}\text { C } & -1.90224800 & -0.96779400 & -2.07711100\end{array}$

$$
\begin{aligned}
& \begin{array}{llll}
\text { C } & -1.17766300 & 0.32342600 & -2.47055700
\end{array} \\
& \text { H } \quad-2.72600100 \quad-1.20748400 \quad-2.76797400 \\
& \mathrm{H} \quad-1.19259800 \quad-1.81169200 \quad-2.11096500 \\
& \mathrm{H} \quad-0.66686100 \quad 0.16857600 \quad-3.45471200 \\
& \mathrm{H} \quad-1.93175000 \quad 1.12314700 \quad-2.67002900 \\
& \mathrm{~N} \quad-0.25662900 \quad 0.70426800 \quad-1.43108000 \\
& \begin{array}{llll}
\text { C } & 0.48370900 & 1.88981700 & -1.74429600
\end{array} \\
& \mathrm{H} \quad-0.16523300 \quad 2.73284800 \quad-2.10408100 \\
& \mathrm{H} \quad 1.23879400 \quad 1.75271200 \quad-2.56472700 \\
& \begin{array}{llll}
\text { Mn } & -0.95222600 & 0.65103500 & 0.53948100
\end{array} \\
& \begin{array}{llll}
\text { C } & -2.06965100 & 2.06355800 & 0.50475500
\end{array} \\
& \begin{array}{llll}
\mathrm{O} & -2.82710800 & 2.94768200 & 0.57831400
\end{array} \\
& \begin{array}{llll}
\text { C } & -1.17908400 & 0.38132100 & 2.28336900
\end{array} \\
& \text { O } \quad-1.25451000 \quad 0.17476900 \quad 3.42876300 \\
& \begin{array}{llll}
\text { C } & -2.36127500 & -2.64272300 & 0.24404800
\end{array} \\
& \text { C } \quad-3.17846200 \quad-3.62407600 \quad-0.60640100 \\
& \begin{array}{llll}
\text { C } & -2.61398800 & -2.86734900 & 1.74073800
\end{array} \\
& \text { H } \quad-1.28869400 \quad-2.83398300 \quad 0.06582100 \\
& \mathrm{H} \quad-3.00077200 \quad-3.50377100 \quad-1.68639700 \\
& \mathrm{H} \quad-2.91571200 \quad-4.66360200 \quad-0.34384100 \\
& \mathrm{H} \quad-4.26110600 \quad-3.51612700 \quad-0.42956500 \\
& \mathrm{H} \quad-2.04352400 \quad-2.16950400 \quad 2.36859300 \\
& \mathrm{H} \quad-3.68081500 \quad-2.76165600 \quad 1.99609000 \\
& \mathrm{H} \quad-2.31933500 \quad-3.89346800 \quad 2.02121500 \\
& \begin{array}{llll}
\text { C } & -4.31790300 & -0.48883400 & -0.41421300
\end{array} \\
& \begin{array}{llll}
\text { C } & -4.66040500 & 0.60964800 & -1.43217800
\end{array} \\
& \begin{array}{llll}
\text { C } & -4.90999000 & -0.14016900 & 0.96043400
\end{array} \\
& \mathrm{H} \quad-4.77662100 \quad-1.42866800 \quad-0.76731000 \\
& \mathrm{H} \quad-4.32922600 \quad 0.36198000 \quad-2.45180700 \\
& \text { H } \quad-5.75466600 \quad 0.74882800 \quad-1.47006300 \\
& \text { H } \quad-4.21749400 \quad 1.57663500 \quad-1.15195700 \\
& \mathrm{H} \quad-4.72038700 \quad-0.91616500 \quad 1.71571600 \\
& \mathrm{H} \quad-4.49328600 \quad 0.80464500 \quad 1.34046500 \\
& \text { H } \quad-6.00365200 \quad-0.01505700 \quad 0.88028100 \\
& \begin{array}{llll}
\text { C } & 1.15655700 & 2.32043900 & -0.48072600
\end{array} \\
& \begin{array}{llll}
\text { C } & 2.37127000 & 3.32522800 & 1.06872500
\end{array} \\
& \text { H } \quad 3.13169600 \quad 3.98055400 \quad 1.48555900 \\
& \mathrm{~N} \quad 0.71802700 \quad 1.86561300 \quad 0.68490600 \\
& \begin{array}{llll}
\mathrm{N} & 2.17115100 & 3.21279900 & -0.29753100
\end{array} \\
& \begin{array}{llll}
\text { C } & 1.46403000 & 2.48040300 & 1.66090100
\end{array} \\
& \mathrm{H} \quad 1.29846200 \quad 2.28389000 \quad 2.71767900 \\
& \text { C } \quad 2.84253200 \quad 3.97968300 \quad-1.32896700 \\
& \text { H } \quad 2.99487700 \quad 3.35793200 \quad-2.22262300 \\
& \text { H } \quad 2.25812900 \quad 4.86939200 \quad-1.61655800
\end{aligned}
$$




$$
\begin{array}{cccc}
\mathrm{H} & 3.82459700 & 4.30712200 & -0.96099600 \\
\mathrm{H} & 1.02368300 & -0.52619500 & -0.52539400 \\
\mathrm{H} & -0.08917900 & -0.73955000 & 0.71078500 \\
\mathrm{C} & 4.93060400 & -1.89281100 & 1.96373500 \\
\mathrm{C} & 5.14419500 & -2.16624900 & 0.62628400 \\
\mathrm{C} & 4.14337000 & -1.87340600 & -0.33882800 \\
\mathrm{C} & 2.92194900 & -1.27687900 & 0.11099000 \\
\mathrm{C} & 2.70628100 & -1.02438000 & 1.48345400 \\
\mathrm{C} & 3.70539300 & -1.32893600 & 2.39130900 \\
\mathrm{H} & 5.21607400 & -2.61068500 & -2.08214000 \\
\mathrm{H} & 5.70490300 & -2.12511700 & 2.69877300 \\
\mathrm{H} & 6.08265700 & -2.61474400 & 0.29080400 \\
\mathrm{C} & 4.29071400 & -2.15204400 & -1.72507800 \\
\mathrm{H} & 1.74136300 & -0.61264600 & 1.78363400 \\
\mathrm{H} & 3.53922100 & -1.13995100 & 3.45447000 \\
\mathrm{C} & 2.11597700 & -1.20855300 & -2.12508200 \\
\mathrm{C} & 3.26957800 & -1.83590800 & -2.60913400 \\
\mathrm{H} & 1.29613200 & -0.87177000 & -2.75973100 \\
\mathrm{H} & 3.35889000 & -2.03694400 & -3.67768300 \\
\mathrm{~N} & 1.95879800 & -0.95790100 & -0.82184500
\end{array}
$$

\section{ts-H2-II}

P $\quad-1.78757200 \quad 0.06885500 \quad-0.46692500$

$$
\begin{array}{llll}
\text { C } & -0.65740900 & -0.09824200 & -1.96790400
\end{array}
$$$$
\text { C } \quad 0.67457000 \quad-0.70702700 \quad-1.47618400
$$$$
\mathrm{H} \quad-0.48958000 \quad 0.90790300 \quad-2.38169400
$$$$
\mathrm{H} \quad-1.12596300 \quad-0.69676900 \quad-2.76629700
$$$$
\mathrm{H} \quad 0.57580500 \quad-1.80284400 \quad-1.40862300
$$$$
\mathrm{H} \quad 1.47677600 \quad-0.50321600 \quad-2.20628300
$$$$
\mathrm{N} \quad 0.99068700 \quad-0.15080900 \quad-0.15134400
$$$$
\begin{array}{llll}
\text { C } & 1.94070400 & 0.96291300 & -0.23686000
\end{array}
$$$$
\mathrm{H} \quad 1.94987800 \quad 1.49531500 \quad 0.72575200
$$$$
\text { H } \quad 1.66073500 \quad 1.69627200 \quad-1.01654800
$$$$
\text { Mn } \quad-0.31637800 \quad-0.43525200 \quad 1.16869400
$$$$
\begin{array}{llll}
\text { C } & -0.58259400 & -2.21938700 & 0.85340300
\end{array}
$$$$
\text { O } \quad-0.75489000 \quad-3.35730100 \quad 0.79331600
$$$$
\begin{array}{llll}
\text { C } & -0.15857500 & 1.13692600 & 2.10806200
\end{array}
$$$$
\begin{array}{llll}
\mathrm{O} & -0.17328500 & 2.04335700 & 2.81993900
\end{array}
$$$$
\text { C } \quad-3.17388800-1.14712900 \quad-0.86434300
$$$$
\begin{array}{llll}
\text { C } & -4.04700500 & -0.73678200 & -2.05862400
\end{array}
$$$$
\begin{array}{llll}
\text { C } & -4.02772800 & -1.55611200 & 0.34400400
\end{array}
$$$$
\mathrm{H} \quad-2.58839900 \quad-2.03531600 \quad-1.16190100
$$$$
\text { H } \quad-3.45507500 \quad-0.45632800 \quad-2.94403900
$$$$
\mathrm{H} \quad-4.69638900 \quad-1.57797800 \quad-2.35271500
$$$$
\mathrm{H} \quad-4.70784200 \quad 0.10900700 \quad-1.81066400
$$

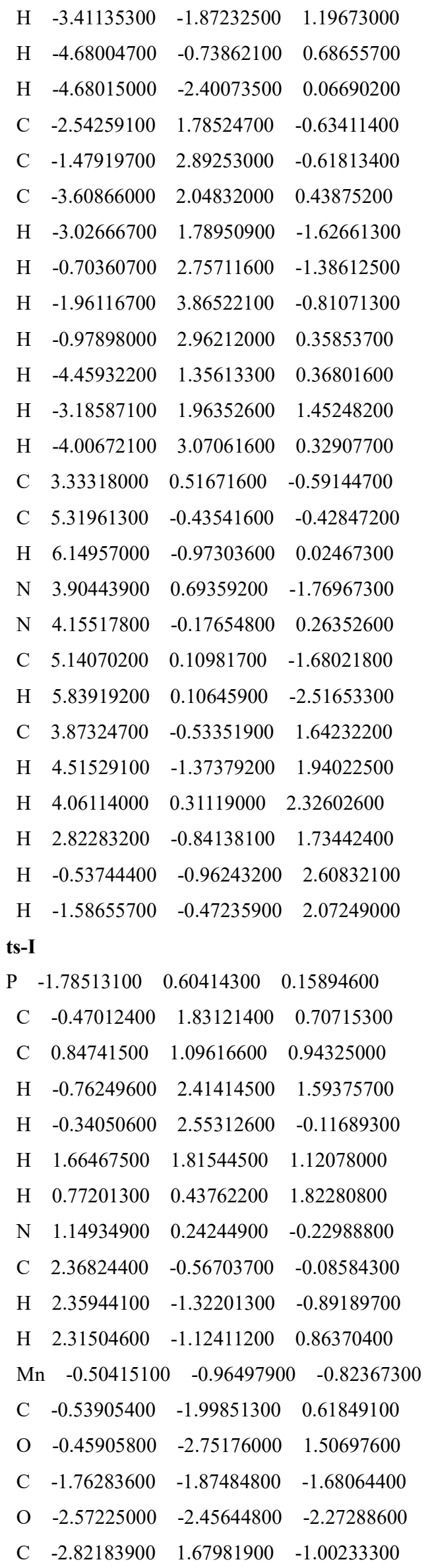




$$
\begin{array}{cccc}
\mathrm{C} & -3.25342900 & 3.03394000 & -0.42200300 \\
\mathrm{C} & -4.01916000 & 0.94136900 & -1.61682500 \\
\mathrm{H} & -2.09428200 & 1.86458100 & -1.81447900 \\
\mathrm{H} & -2.41002100 & 3.62516500 & -0.03483500 \\
\mathrm{H} & -3.74313000 & 3.63789400 & -1.20451800 \\
\mathrm{H} & -3.98544500 & 2.91389500 & 0.39335300 \\
\mathrm{H} & -3.74811600 & -0.04477200 & -2.01838700 \\
\mathrm{H} & -4.82801400 & 0.80266800 & -0.88163200 \\
\mathrm{H} & -4.43718500 & 1.53686400 & -2.44538600 \\
\mathrm{C} & -2.89031600 & 0.32336800 & 1.66358300 \\
\mathrm{C} & -2.09664800 & 0.17753200 & 2.97031800 \\
\mathrm{C} & -3.79478700 & -0.90183000 & 1.45166300 \\
\mathrm{H} & -3.52502100 & 1.22302100 & 1.74511300 \\
\mathrm{H} & -1.49858900 & 1.06964100 & 3.20953900 \\
\mathrm{H} & -2.79539900 & 0.02283200 & 3.80949900 \\
\mathrm{H} & -1.42655300 & -0.69508700 & 2.94071500 \\
\mathrm{H} & -4.37650600 & -0.84952000 & 0.52088800 \\
\mathrm{H} & -3.20187600 & -1.82812600 & 1.42214800 \\
\mathrm{H} & -4.50867200 & -0.98805600 & 2.28802800 \\
\mathrm{C} & 3.62023000 & 0.26486800 & -0.17352100 \\
\mathrm{C} & 5.75252500 & 0.82925600 & -0.15055800 \\
\mathrm{H} & 6.82508300 & 0.72227400 & -0.00682200 \\
\mathrm{~N} & 3.65299100 & 1.51502600 & -0.58846300 \\
\mathrm{~N} & 4.87427800 & -0.20740900 & 0.11002200 \\
\mathrm{C} & 4.97569600 & 1.88056300 & -0.57661000 \\
\mathrm{H} & 5.30084900 & 2.87740800 & -0.87116000 \\
\mathrm{H} & 5.22000700 & -1.54737200 & 0.54863100 \\
\mathrm{H} & -3.65433900 & -1.82458800 & 1.45155000 \\
\mathrm{H} & -24191100 & -0.96331000 & -2.29906500
\end{array}
$$$$
\text { ts-II }
$$$$
\text { P } \quad 1.73161400 \quad-0.65801700 \quad 0.17610800
$$$$
\begin{array}{llll}
\text { C } & 0.38431700 & -1.89050500 & 0.64661500
\end{array}
$$$$
\text { C } \quad-0.88503200 \quad-1.15445000 \quad 1.07045900
$$$$
\mathrm{H} \quad 0.70166800 \quad-2.58839200 \quad 1.43791500
$$$$
\mathrm{H} \quad 0.17094700 \quad-2.50114300 \quad-0.24725000
$$$$
\text { H } \quad-1.73183100 \quad-1.85963000 \quad 1.13209300
$$$$
\text { H } \quad-0.75648800 \quad-0.69827600 \quad 2.06422900
$$$$
\mathrm{N} \quad-1.20177200 \quad-0.05182400 \quad 0.12246000
$$$$
\begin{array}{llll}
\text { C } & -2.47734000 & 0.61860300 & 0.43914700
\end{array}
$$$$
\mathrm{H} \quad-2.46829100 \quad 1.59913600 \quad-0.06201700
$$$$
\mathrm{H} \quad-2.51501700 \quad 0.82016600 \quad 1.52292600
$$

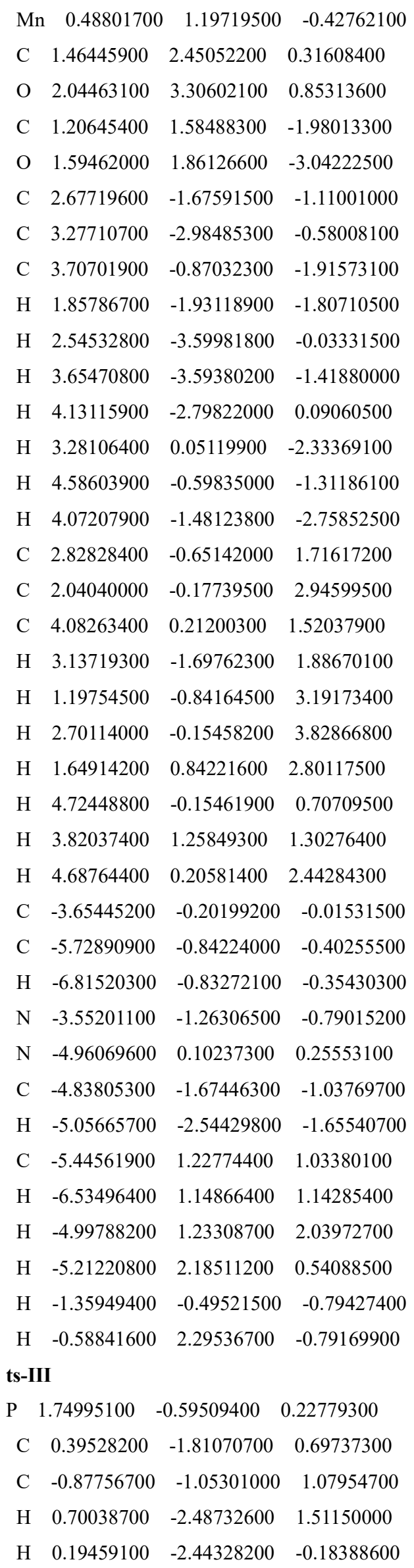




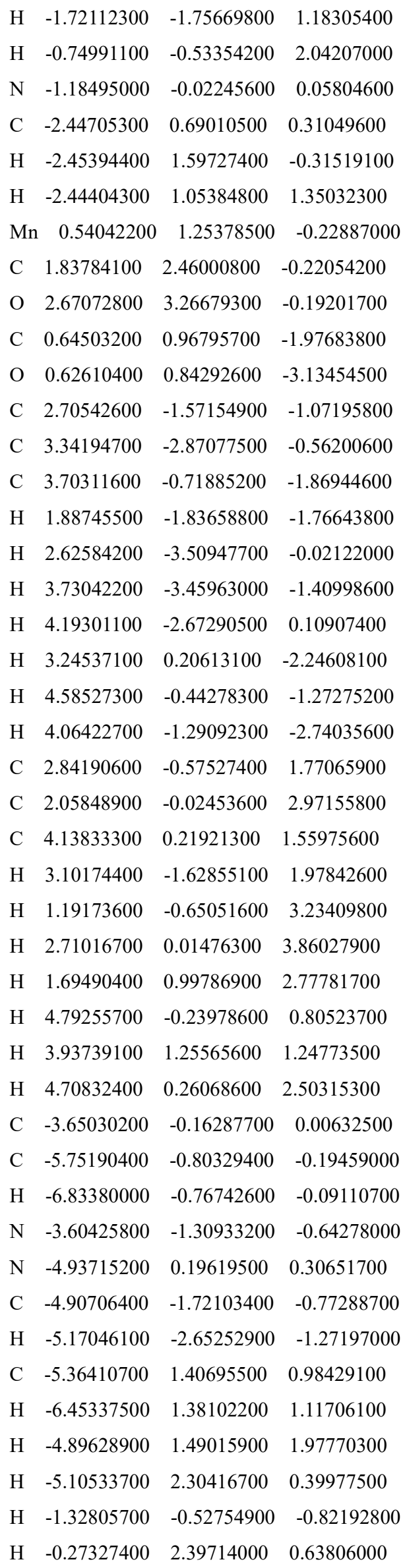

2-ts

$\begin{array}{llll}\text { P } & 2.29216200 & 0.03578100 & -0.31046800\end{array}$

$\begin{array}{llll}\text { C } & 2.43914200 & -1.11309700 & -1.77657500\end{array}$

$\begin{array}{llll}\text { C } & 1.20342900 & -2.01462700 & -1.81180800\end{array}$

H $3.37694300 \quad-1.68937900 \quad-1.77997200$

H $\quad 2.44257100 \quad-0.47135700 \quad-2.67308900$

H $\quad 1.19488400 \quad-2.56740600 \quad-2.77754900$

H $\quad 1.29731600 \quad-2.79879600 \quad-1.02252900$

$\mathrm{N} \quad 0.00000000 \quad-1.23255600 \quad-1.65697300$

H $\quad 0.00000000 \quad 0.31322300 \quad-1.72108100$

$\begin{array}{llll}\text { C } & -1.20343000 & -2.01462600 & -1.81180800\end{array}$

C $\quad-2.43914200-1.11309600 \quad-1.77657500$

$\mathrm{H} \quad-1.29731700 \quad-2.79879500 \quad-1.02253000$

$\mathrm{H} \quad-1.19488500 \quad-2.56740600 \quad-2.77754900$

$\mathrm{H} \quad-2.44257100 \quad-0.47135600 \quad-2.67308900$

$\mathrm{H} \quad-3.37694400 \quad-1.68937800 \quad-1.77997200$

$\begin{array}{llll}\mathrm{P} & -2.29216200 & 0.03578100 & -0.31046800\end{array}$

$\begin{array}{llll}\text { Mn } & 0.00000000 & 0.11073800 & 0.00119900\end{array}$

C $\quad-0.00000100 \quad-1.11217300 \quad 1.29769500$

$\begin{array}{llll}\text { O } & -0.00000100 & -1.91805000 & 2.13683300\end{array}$

$\begin{array}{llll}\text { C } & 0.00000100 & 1.47926300 & 1.14765200\end{array}$

$\begin{array}{llll}\mathrm{O} & 0.00000500 & 2.37019000 & 1.89540300\end{array}$

$\begin{array}{llll}\text { C } & 3.14777800 & 1.58787900 & -0.96920800\end{array}$

C $\quad 4.55031700 \quad 1.34755200 \quad-1.54502000$

$\begin{array}{llll}\text { C } & 3.15237700 & 2.76623400 & 0.01413800\end{array}$

H $\quad 2.47431300 \quad 1.85545900 \quad-1.80333700$

H $\quad 4.57014800 \quad 0.54584500 \quad-2.29833200$

H $\quad 4.91794900 \quad 2.26621900 \quad-2.03280200$

H $\quad 5.27794000 \quad 1.09144300 \quad-0.75764700$

$\begin{array}{llll}\mathrm{H} & 2.16332200 & 2.95388200 & 0.45375900\end{array}$

H $\quad 3.86572500 \quad 2.60474100 \quad 0.83815500$

H $3.46918700 \quad 3.68503500 \quad-0.50741400$

C $3.46931400 \quad-0.63376000 \quad 1.00564300$

$\begin{array}{llll}\text { C } & 3.43636900 & -2.16476200 & 1.12690500\end{array}$

$\begin{array}{llll}\text { C } & 3.20888200 & 0.01897500 & 2.37299200\end{array}$

H $\quad 4.47844500 \quad-0.34394900 \quad 0.66532400$

H $3.70744800 \quad-2.67092600 \quad 0.18905100$

$\mathrm{H} \quad 4.16189700 \quad-2.48604300 \quad 1.89316500$

H $\quad 2.44706600 \quad-2.52865300 \quad 1.44092700$

H $\quad 3.19823200 \quad 1.11708800 \quad 2.32975900$

H $\quad 2.24450200 \quad-0.30687700 \quad 2.79004800$

H $3.99638000 \quad-0.27974700 \quad 3.08531000$

$\begin{array}{llll}\text { C } & -3.14777900 & 1.58788000 & -0.96920700\end{array}$

$\begin{array}{llll}\text { C } & -3.15237800 & 2.76623400 & 0.01413900\end{array}$ 


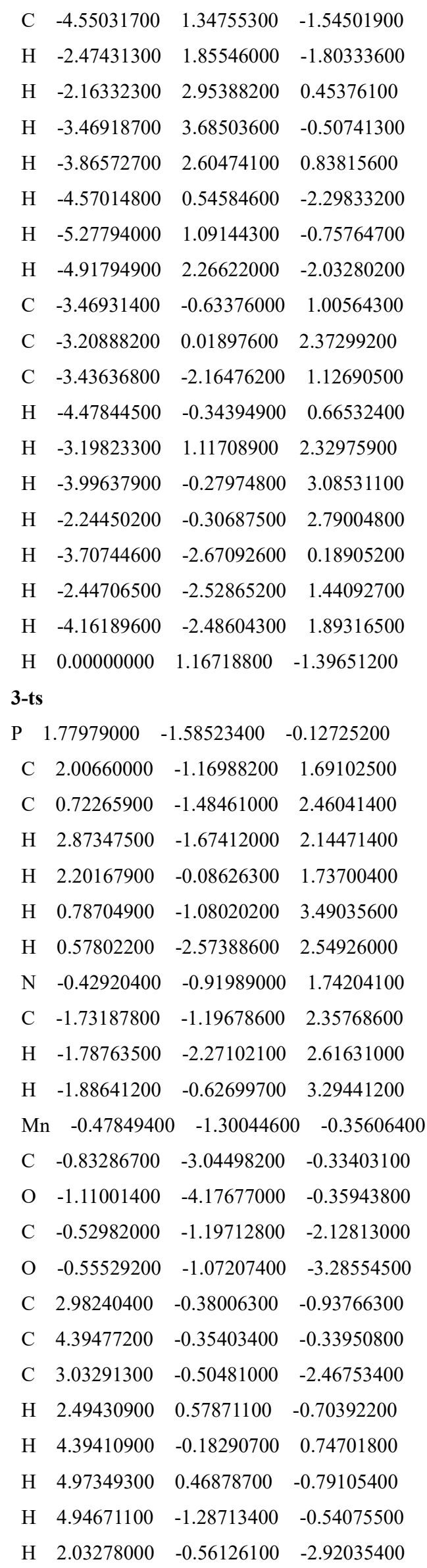

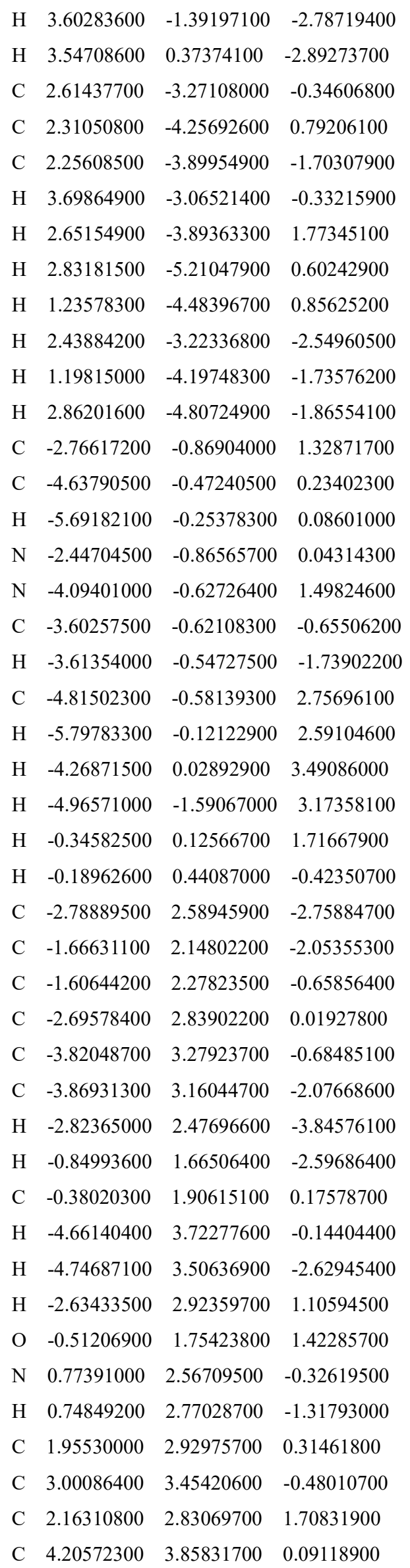




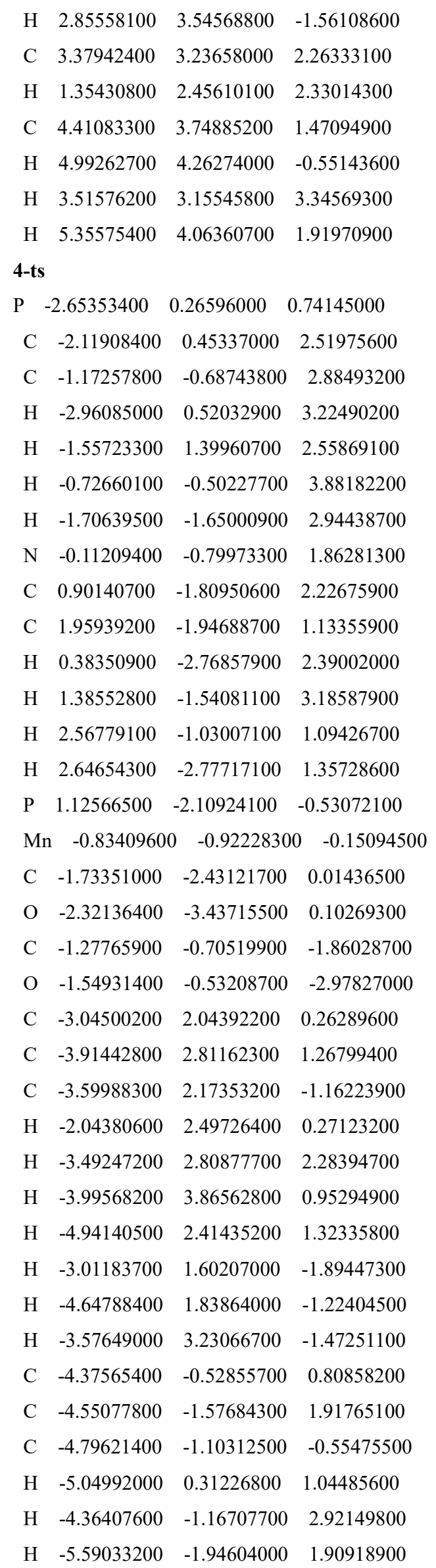

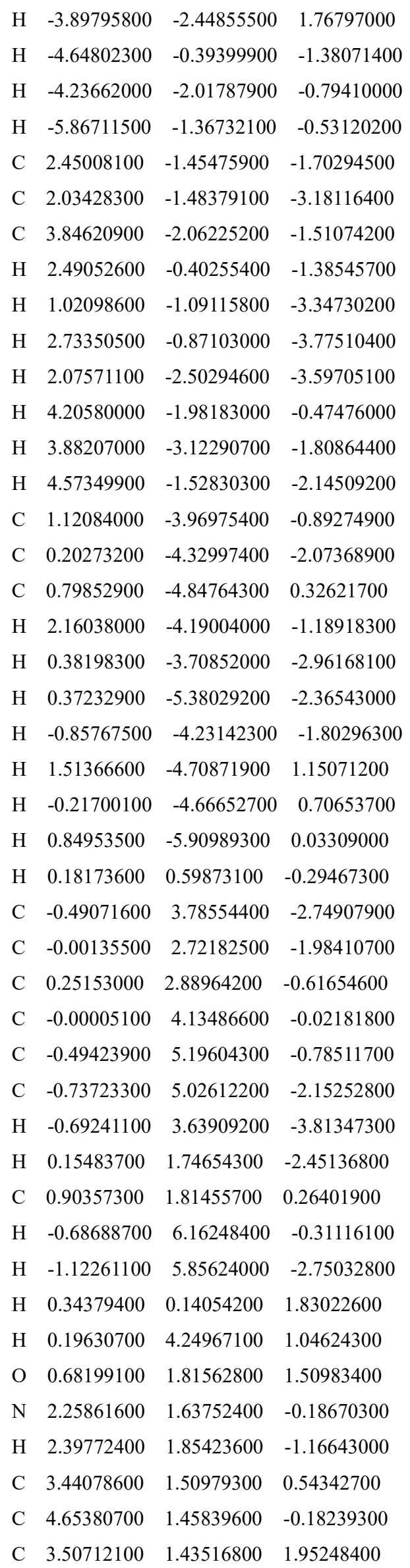




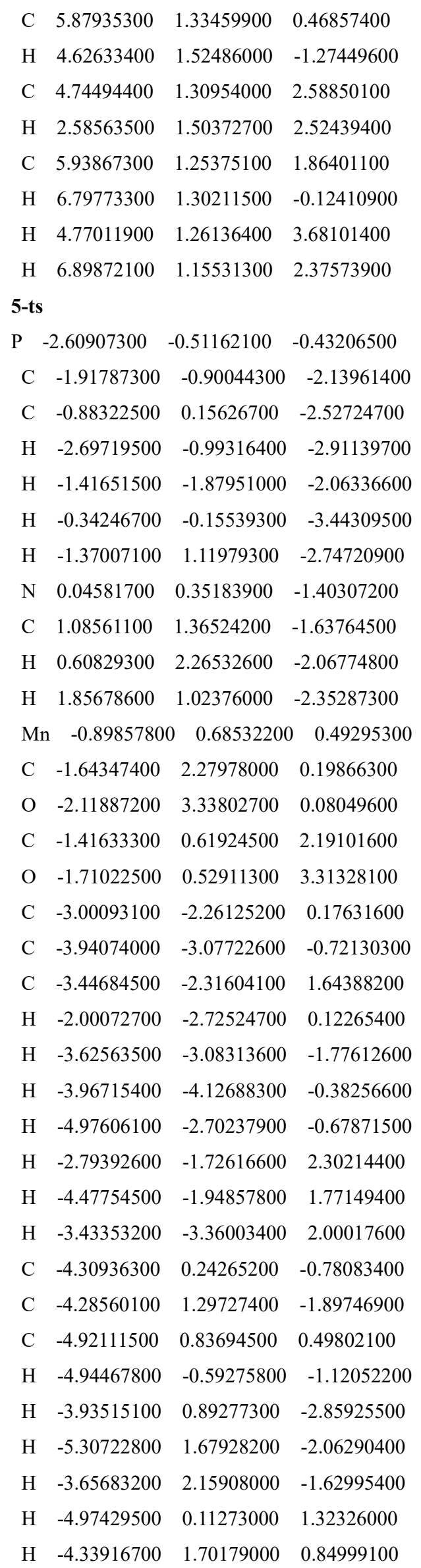

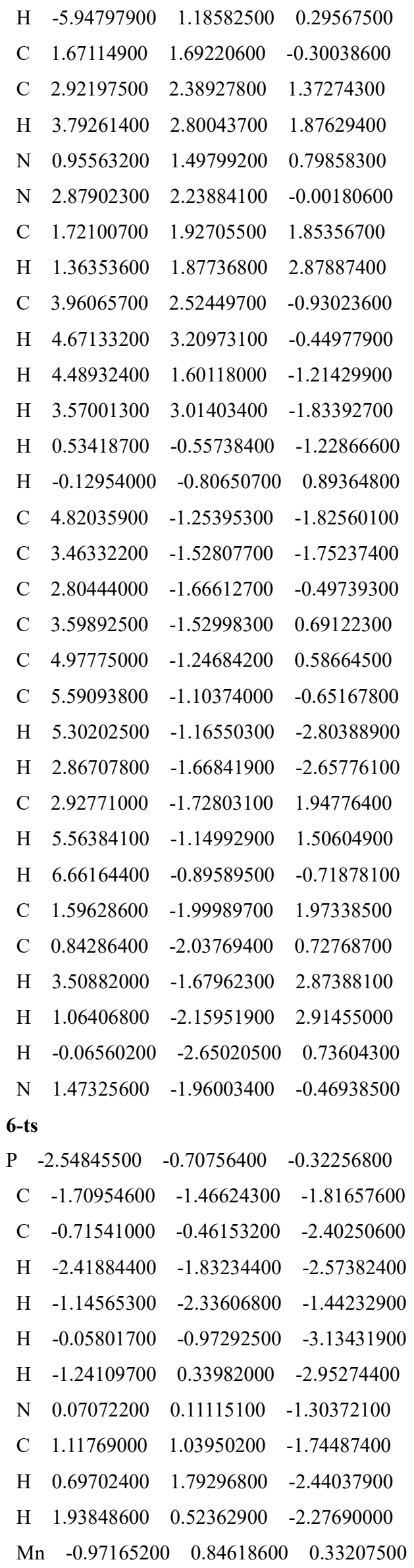




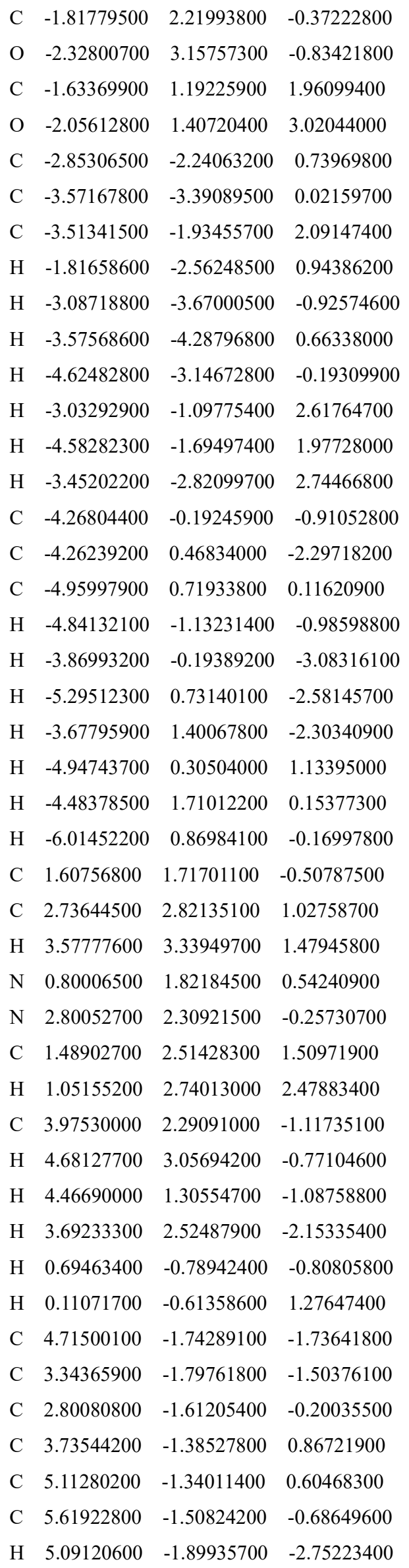

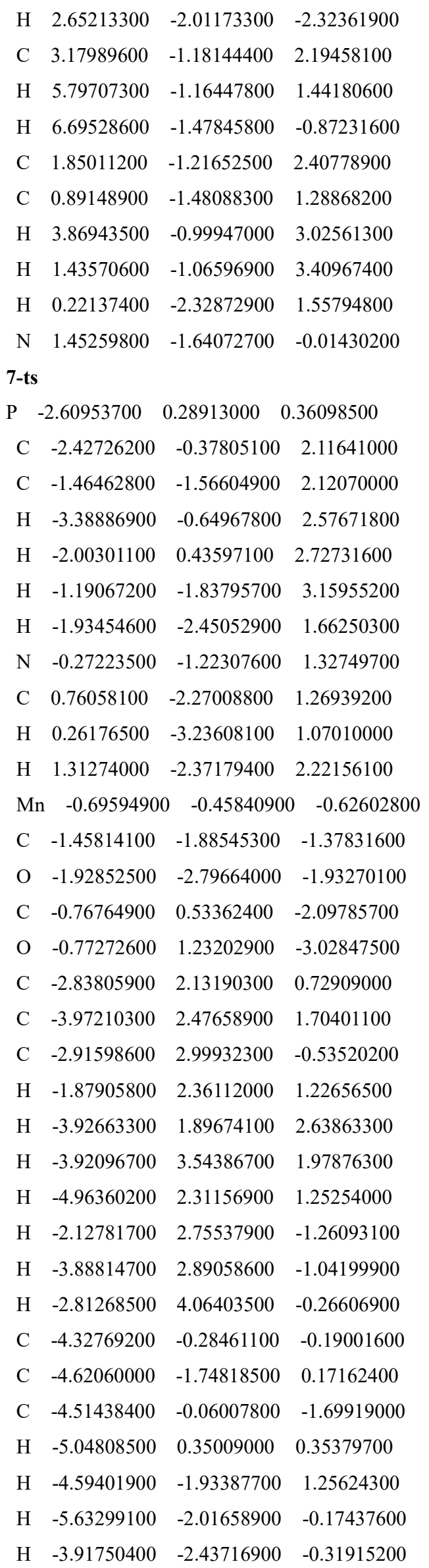




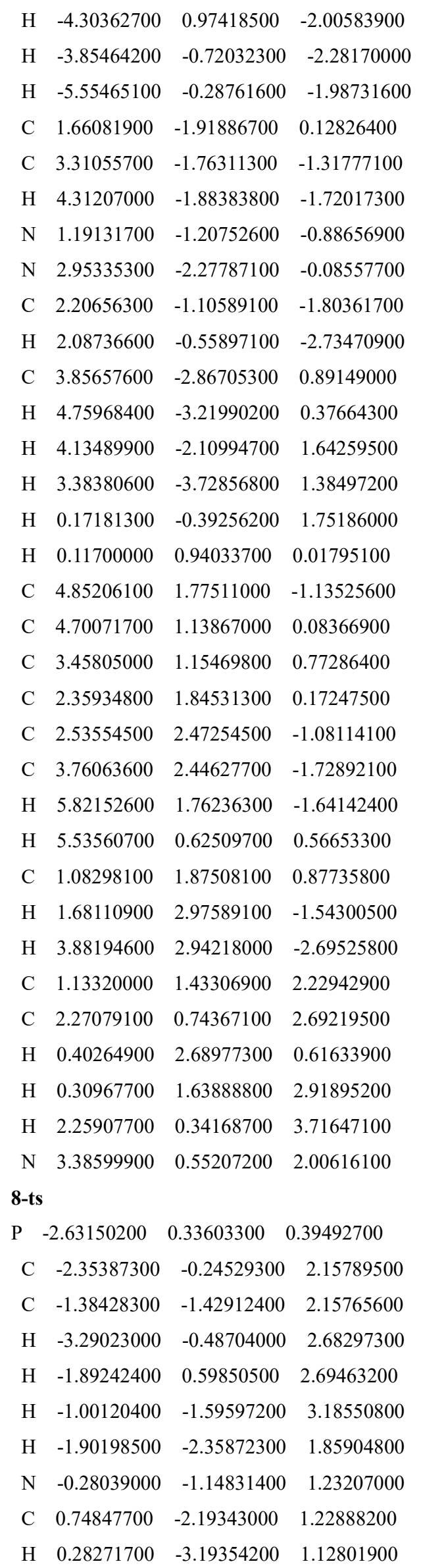

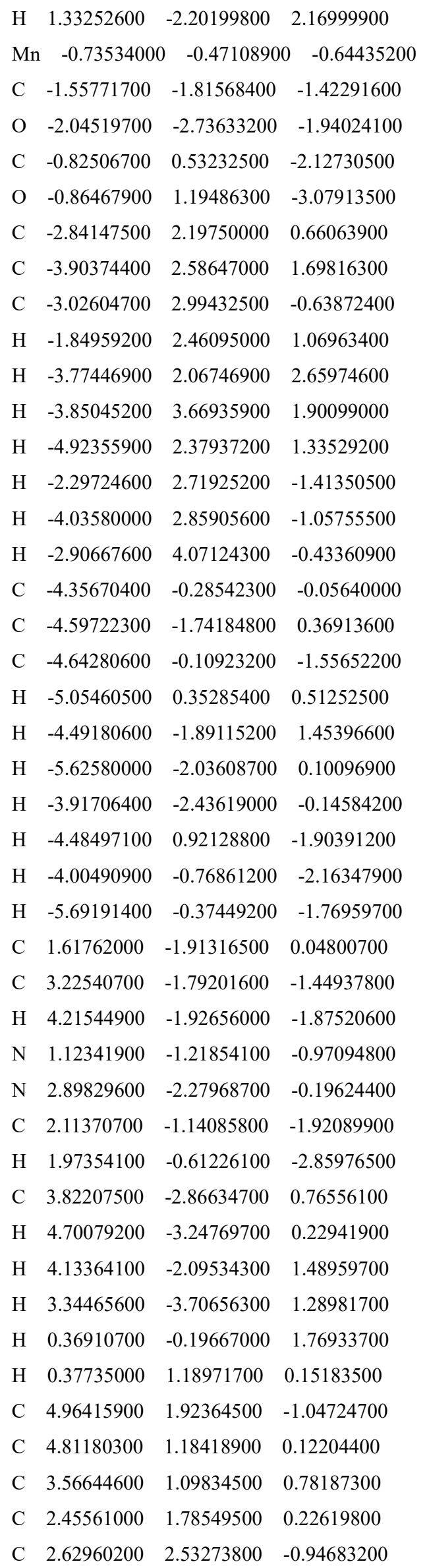




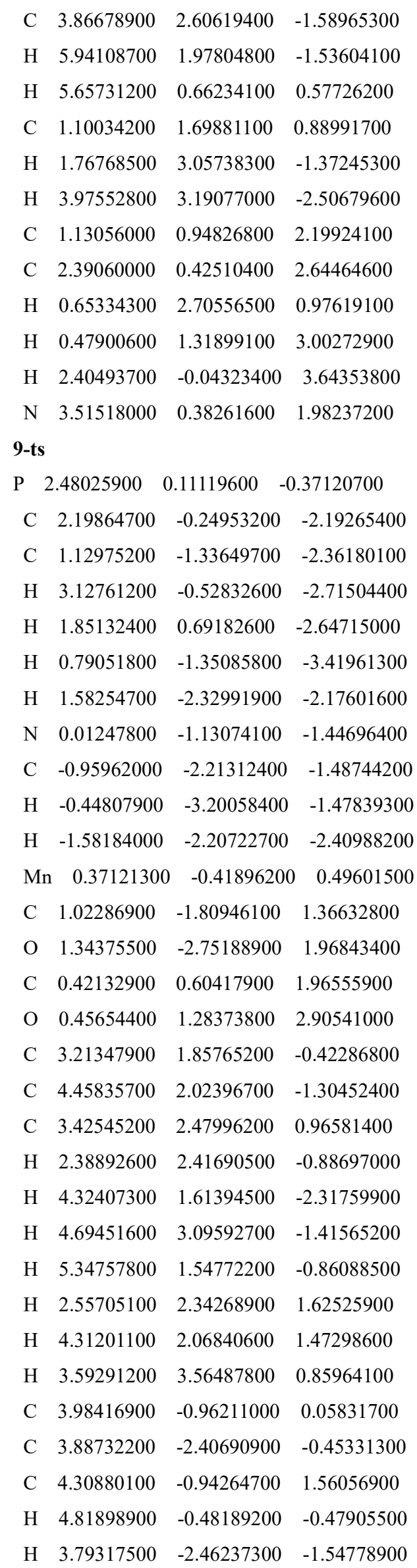

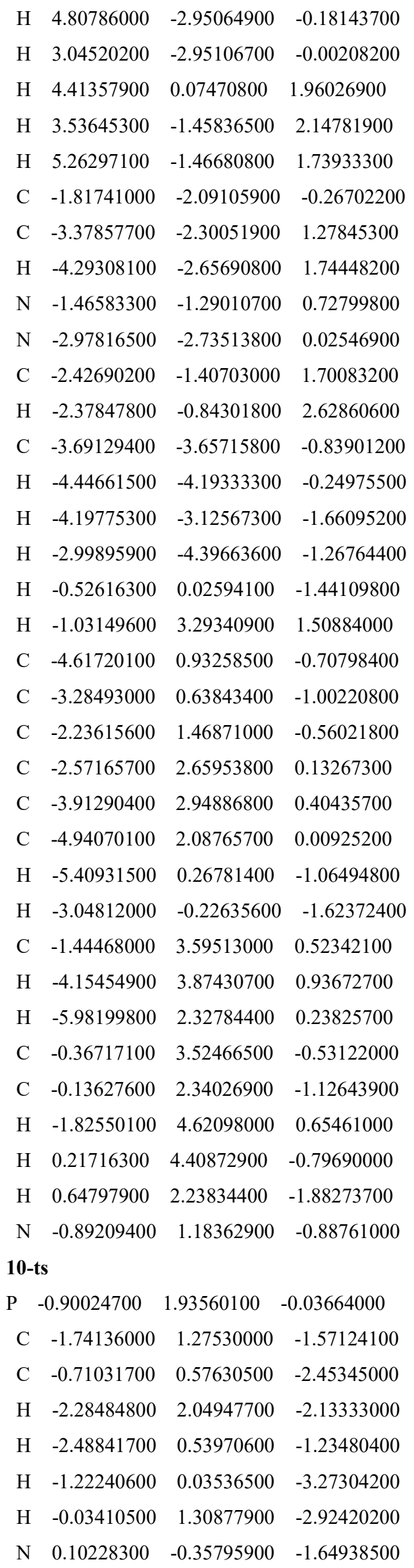




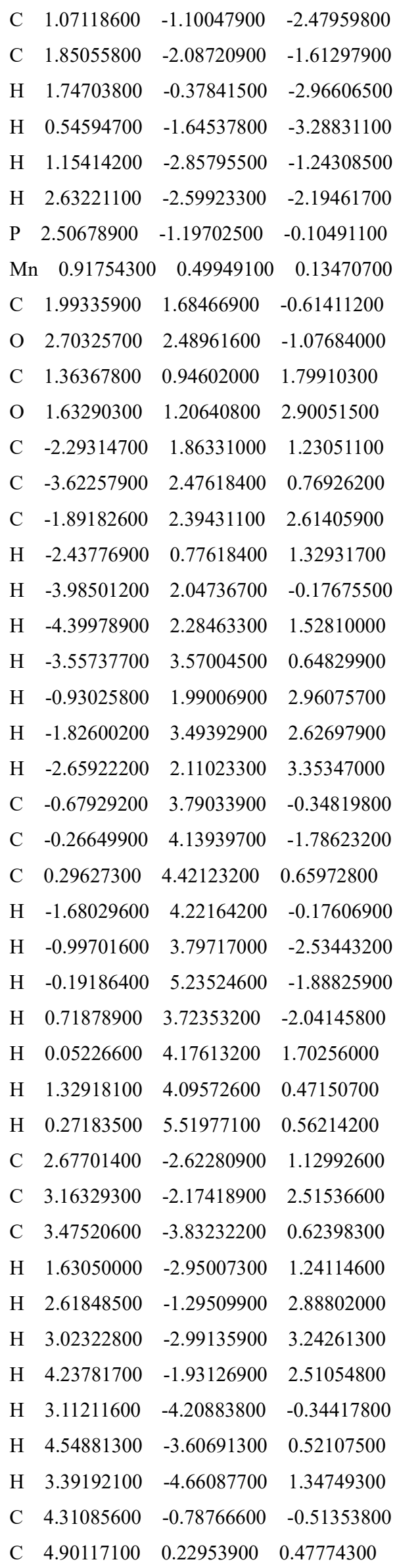

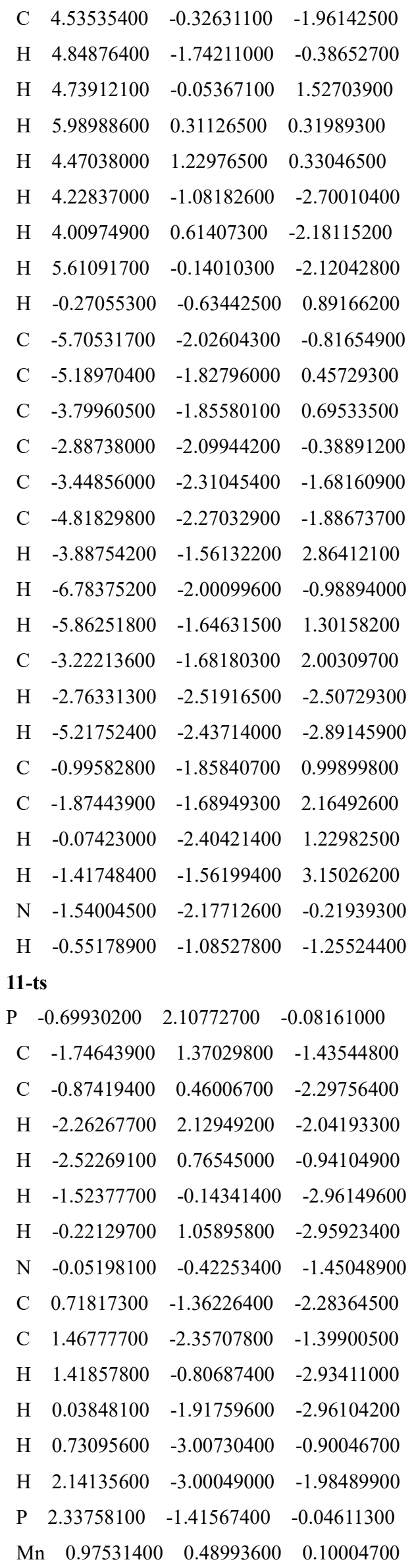




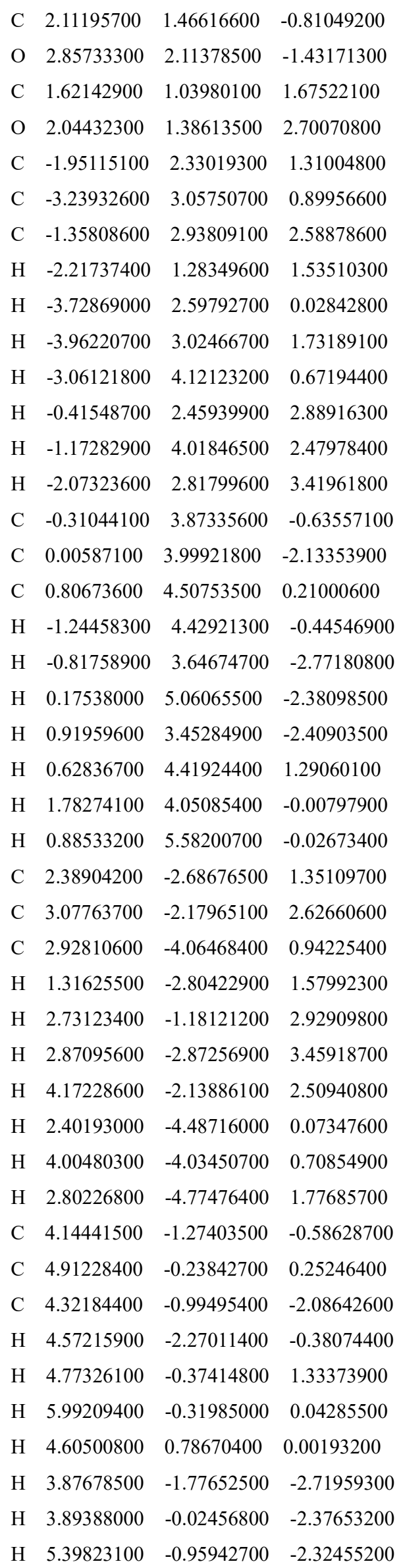

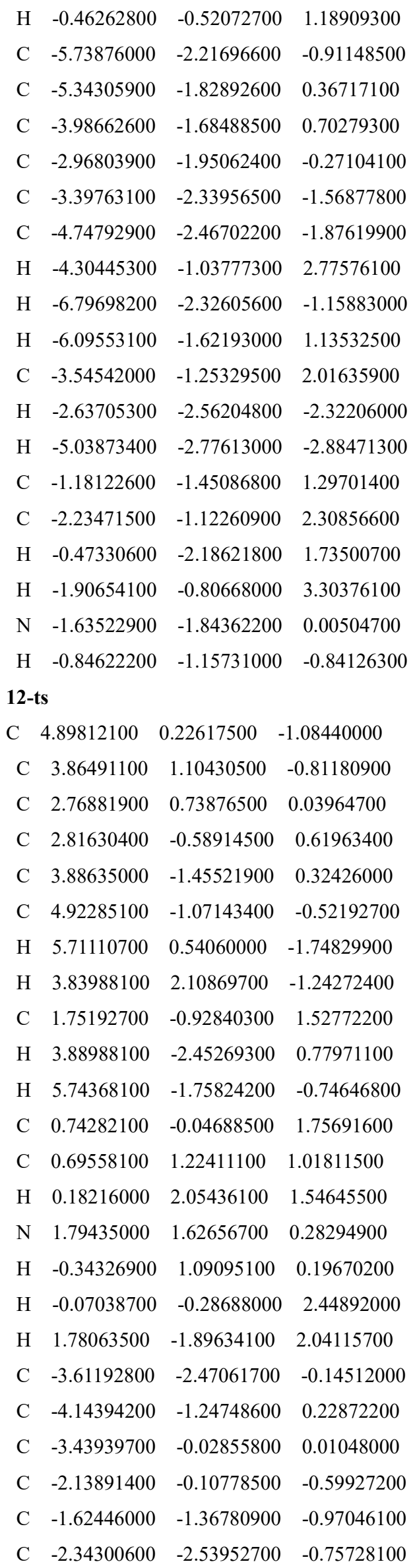




$$
\begin{array}{llll}
\mathrm{H} & -4.18103000 & -3.38937700 & 0.03217600 \\
\mathrm{H} & -5.13018900 & -1.16956100 & 0.69403300 \\
\mathrm{C} & -1.37306200 & 1.12915800 & -0.77088300 \\
\mathrm{H} & -0.63191500 & -1.41152900 & -1.43041300 \\
\mathrm{H} & -1.92307700 & -3.50541900 & -1.05217900 \\
\mathrm{C} & -2.12245500 & 2.33495600 & -0.55565300 \\
\mathrm{C} & -3.37733300 & 2.26481100 & 0.04198600 \\
\mathrm{~N} & -4.04100900 & 1.14684700 & 0.35341300 \\
\mathrm{H} & -0.59572900 & 1.13171700 & -1.55180100 \\
\mathrm{H} & -1.68215200 & 3.30694100 & -0.79376300 \\
\mathrm{H} & -3.90209900 & 3.20640600 & 0.26790400
\end{array}
$$

\section{3-ts}

$\begin{array}{llll}\text { C } & -2.79827100 & -2.05760700 & 1.48309200\end{array}$

$\begin{array}{llll}\text { C } & -1.84223100 & -1.29717500 & 0.81727700\end{array}$

$\begin{array}{llll}\text { C } & -2.19786900 & -0.12598800 & 0.09882700\end{array}$

$\begin{array}{llll}\text { C } & -3.57225100 & 0.26055700 & 0.07666200\end{array}$

C $\quad-4.50871700 \quad-0.52803000 \quad 0.75295000$

$\begin{array}{llll}\text { C } & -4.14847800 & -1.68227600 & 1.45799700\end{array}$

$\mathrm{H} \quad-2.48707700 \quad-2.95484400 \quad 2.02841000$

$\mathrm{H} \quad-0.78749700 \quad-1.58318000 \quad 0.83201700$

$\begin{array}{llll}\text { C } & -4.01685900 & 1.51978400 & -0.66213800\end{array}$

$\mathrm{H} \quad-5.56169300 \quad-0.22175200 \quad 0.72632200$

$\mathrm{H} \quad-4.90588200 \quad-2.27580500 \quad 1.97812900$

C $\quad-2.86278700 \quad 2.16934900 \quad-1.38608200$

C $\quad-1.60193500 \quad 1.68604500 \quad-1.28010000$

$\mathrm{N} \quad-1.22345500 \quad 0.58780900 \quad-0.55395400$

$\mathrm{H} \quad-4.84093500 \quad 1.26754100 \quad-1.36584800$

$\mathrm{H} \quad-3.05151500 \quad 3.05706900 \quad-1.99697100$

$\mathrm{H} \quad-0.77483200 \quad 2.18152300 \quad-1.80468200$

$\begin{array}{lllll}\mathrm{H} & -0.02853600 & 0.20983400 & -0.59841500\end{array}$

$\mathrm{H} \quad-4.48817800 \quad 2.22802200 \quad 0.05744700$

$\begin{array}{llll}\text { C } & 3.15091400 & 1.79330400 & 1.66994900\end{array}$

$\begin{array}{llll}\text { C } & 2.08810300 & 1.30829100 & 0.91851200\end{array}$

$\begin{array}{llll}\text { C } & 2.25646600 & 0.22627200 & -0.00255000\end{array}$

$\begin{array}{llll}\text { C } & 3.57415300 & -0.36296900 & -0.06631700\end{array}$

$\begin{array}{llll}\text { C } & 4.62096700 & 0.14914400 & 0.71142000\end{array}$

C $\quad 4.43858800 \quad 1.23279300 \quad 1.57509500$

$\begin{array}{llll}\mathrm{H} & 2.97631900 & 2.63442500 & 2.35057100\end{array}$

H $\quad 1.09434200 \quad 1.75363300 \quad 1.00960200$

$\begin{array}{llll}\text { C } & 3.71160200 & -1.55383800 & -0.89214200\end{array}$

$\mathrm{H} \quad 5.60445200 \quad-0.33141700 \quad 0.63657700$

H $\quad 5.26854700 \quad 1.62548000 \quad 2.16870400$

C $\quad 2.70366600 \quad-1.98205700 \quad-1.67563600$

C $\quad 1.43060100 \quad-1.18043700 \quad-1.80686600$

$$
\begin{array}{llll}
\mathrm{H} & 0.55680700 & -1.86253100 & -1.86205300 \\
\mathrm{~N} & 1.22194300 & -0.22940100 & -0.73092100 \\
\mathrm{H} & 4.64907100 & -2.12016100 & -0.83098900 \\
\mathrm{H} & 2.80333000 & -2.89939300 & -2.26783200 \\
\mathrm{H} & 1.42989500 & -0.66826900 & -2.80658000
\end{array}
$$




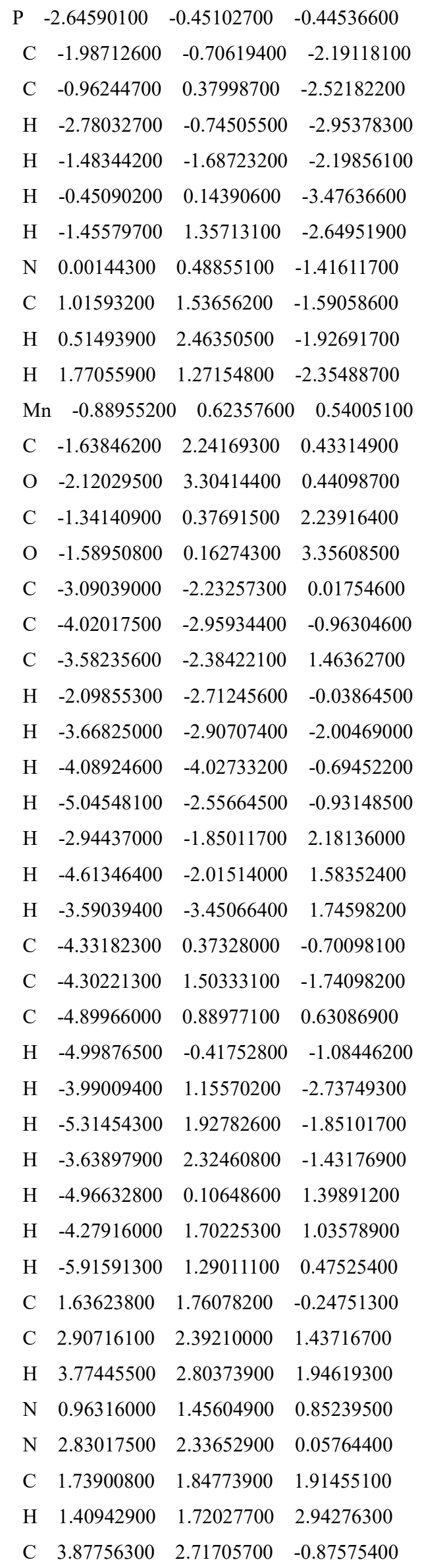

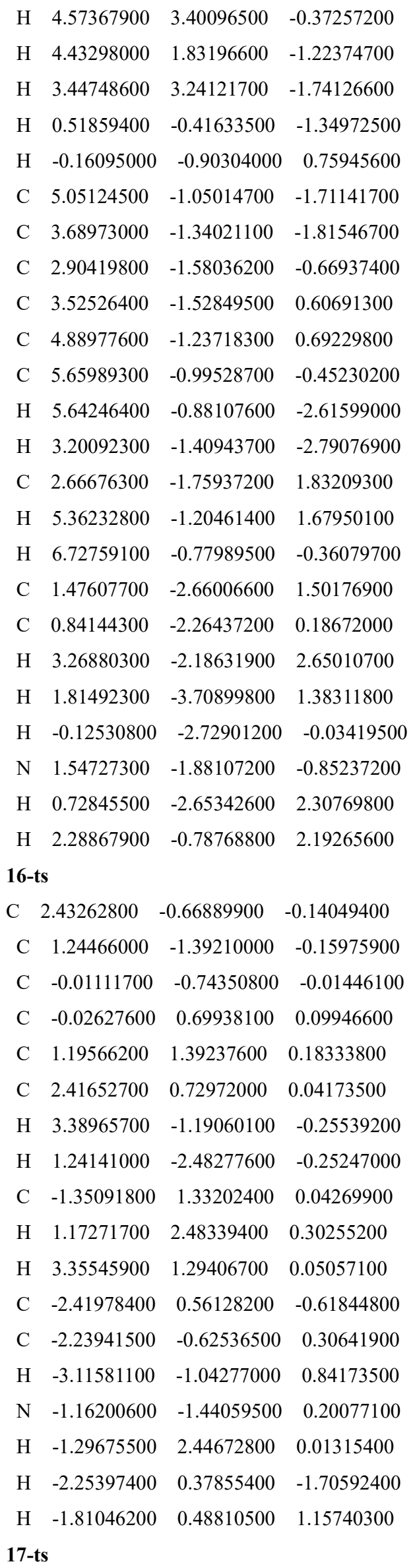




$$
\begin{aligned}
& \begin{array}{lrrr}
\text { C } & -5.48170200 & -0.08602300 & -0.37117800
\end{array} \\
& \text { C } \quad-4.45904400 \quad-1.02804900 \quad-0.31154300 \\
& \text { C } \quad-3.14510400 \quad-0.68174100 \quad 0.10247900 \\
& \text { C } \quad-2.90419300 \quad 0.68664500 \quad 0.44998600 \\
& \text { C } \quad-3.94612300 \quad 1.61404400 \quad 0.38692100 \\
& \text { C } \quad-5.23700500 \quad 1.24978200 \quad-0.01953000 \\
& \text { H } \quad-6.48300400 \quad-0.39222100 \quad-0.69290900 \\
& \mathrm{H} \quad-4.63185700 \quad-2.07417300 \quad-0.57824700 \\
& \text { C } \quad-1.49598600 \quad 1.07555300 \quad 0.84013600 \\
& \mathrm{H} \quad-3.73977000 \quad 2.65529900 \quad 0.66308600 \\
& \mathrm{H} \quad-6.03676600 \quad 1.99493200 \quad-0.06040700 \\
& \text { C } \quad-0.76175100 \quad-0.11066900 \quad 1.47343000 \\
& \text { C } \quad-0.98494300 \quad-1.36762300 \quad 0.63878100 \\
& \mathrm{H} \quad-0.35209100 \quad-2.22747900 \quad 0.92085600 \\
& \mathrm{~N} \quad-2.19165600 \quad-1.67049500 \quad 0.15392400 \\
& \mathrm{H} \quad-0.01912200 \quad-1.09946700 \quad-0.43376000 \\
& \mathrm{H} \quad 0.31517800 \quad 0.09248900 \quad 1.58199300 \\
& \mathrm{H} \quad-0.93475500 \quad 1.39300000 \quad-0.06009300 \\
& \begin{array}{llll}
\text { C } & 5.16472200 & -0.27657100 & 1.02414900
\end{array} \\
& \text { C } \quad 4.15813600 \quad-1.13349500 \quad 0.61748400 \\
& \text { C } \quad 3.05562300 \quad-0.67999500 \quad-0.18416300 \\
& \text { C } \quad 3.07688000 \quad 0.71924700 \quad-0.56964400 \\
& \text { C } \quad 4.12046000 \quad 1.56071300 \quad-0.14260500 \\
& \begin{array}{llll}
\text { C } & 5.16058400 & 1.08871900 & 0.65402400
\end{array} \\
& \text { H } \quad 5.98050000 \quad-0.66279600 \quad 1.64573600 \\
& \mathrm{H} \quad 4.15787100 \quad-2.18917800 \quad 0.90179200 \\
& \text { C } \quad 2.01567900 \quad 1.15481600 \quad-1.44194100 \\
& \mathrm{H} \quad 4.10162800 \quad 2.61235000 \quad-0.45384700 \\
& \text { H } \quad 5.96097800 \quad 1.75707300 \quad 0.98342100 \\
& \begin{array}{llll}
\text { C } & 1.02797800 & 0.29476500 & -1.80207700
\end{array} \\
& \text { C } \quad 0.99095300 \quad-1.07127000 \quad-1.23714100 \\
& \text { N } \quad 2.10814400 \quad-1.54736500 \quad-0.56031600 \\
& \mathrm{H} \quad 2.03765700 \quad 2.17980700 \quad-1.83092300 \\
& \text { H } \quad 0.22819000 \quad 0.60625300 \quad-2.48244200 \\
& \mathrm{H} \quad 0.53414500 \quad-1.82180100 \quad-1.91384900 \\
& \mathrm{H} \quad-1.15873800 \quad-0.29931100 \quad 2.49177500 \\
& \mathrm{H} \quad-1.50645700 \quad 1.94716700 \quad 1.51715800
\end{aligned}
$$

\section{8-ts}

$$
\begin{array}{cccc}
\text { C } & -2.91954100 & 2.08214500 & -1.48005300 \\
\text { C } & -1.90368300 & 1.29597200 & -0.95230500 \\
\text { C } & -2.17752000 & 0.07761900 & -0.25264400 \\
\text { C } & -3.56310700 & -0.28197200 & -0.10758900 \\
\text { C } & -4.55917800 & 0.53687500 & -0.64411400 \\
\text { C } & -4.26886100 & 1.71669900 & -1.34062300
\end{array}
$$

$$
\begin{aligned}
& \mathrm{H} \quad-2.65660700 \quad 3.00315100 \quad-2.01299000 \\
& \text { H } \quad-0.85691000 \quad 1.59034200 \quad-1.06511800 \\
& \text { C } \quad-3.90560600 \quad-1.54137600 \quad 0.66519300 \\
& \mathrm{H} \quad-5.60627400 \quad 0.23775400 \quad-0.50463100 \\
& \text { H } \quad-5.06917900 \quad 2.33600100 \quad-1.75522000 \\
& \text { C } \quad-2.79178500 \quad-1.88987300 \quad 1.65920100 \\
& \begin{array}{llll}
\text { C } & -1.44453000 & -1.89666800 & 0.92462500
\end{array} \\
& \begin{array}{llll}
\mathrm{N} & -1.15648200 & -0.65595100 & 0.24911100
\end{array} \\
& \text { H } \quad-4.87376400 \quad-1.41843500 \quad 1.18372700 \\
& \begin{array}{llll}
\mathrm{H} & -2.76100500 & -1.13155600 & 2.46232300
\end{array} \\
& \mathrm{H} \quad-0.62288400 \quad-2.10784900 \quad 1.63551400 \\
& \text { H } \quad 0.06312700 \quad-0.18012400 \quad 0.36644400 \\
& \mathrm{H} \quad-4.04041500 \quad-2.39782400 \quad-0.02597400 \\
& \begin{array}{llll}
\text { C } & 3.32459500 & -1.63067700 & -1.74949800
\end{array} \\
& \begin{array}{llll}
\text { C } & 2.20626200 & -1.16727000 & -1.06748900
\end{array} \\
& \begin{array}{llll}
\text { C } & 2.31234800 & -0.14762500 & -0.07038800
\end{array} \\
& \begin{array}{llll}
\text { C } & 3.62900900 & 0.39631800 & 0.15786900
\end{array} \\
& \begin{array}{llll}
\text { C } & 4.73281900 & -0.09266400 & -0.55183400
\end{array} \\
& \begin{array}{llll}
\text { C } & 4.60853600 & -1.11108800 & -1.50178100
\end{array} \\
& \text { H } 3.19648000 \quad-2.42030800 \quad-2.49870600 \\
& \text { H } \quad 1.21434900 \quad-1.57392600 \quad-1.27818000 \\
& \begin{array}{llll}
\text { C } & 3.71960100 & 1.50234000 & 1.10064500
\end{array} \\
& \text { H } \quad 5.71569700 \quad 0.35096500 \quad-0.35006100 \\
& \text { H } \quad 5.48159100 \quad-1.48525100 \quad-2.04314900 \\
& \text { C } \quad 2.64701400 \quad 1.92150500 \quad 1.79746200 \\
& \text { C } \quad 1.31455200 \quad 1.22092400 \quad 1.69454900 \\
& \mathrm{H} \quad 0.50004300 \quad 1.97246600 \quad 1.61083900 \\
& \mathrm{~N} \quad 1.22354200 \quad 0.28710700 \quad 0.58830000 \\
& \mathrm{H} \quad 4.68602600 \quad 2.00904600 \quad 1.21204400 \\
& \text { H } \quad 2.72542500 \quad 2.77026100 \quad 2.48710000 \\
& \mathrm{H} \quad 1.09908300 \quad 0.71024300 \quad 2.66957700 \\
& \mathrm{H} \quad-2.97945500 \quad-2.86789800 \quad 2.13796100 \\
& \text { H } \quad-1.45029500 \quad-2.76224600 \quad 0.21928000 \\
& \text { 19-ts } \\
& \text { C } \quad-5.14719700 \quad 0.63729800 \quad-0.68067000 \\
& \begin{array}{llll}
\text { C } & -4.51084800 & -0.57001600 & -0.39704000
\end{array} \\
& \begin{array}{llll}
\text { C } & -3.21863500 & -0.60830800 & 0.17197300
\end{array} \\
& \begin{array}{llll}
\text { C } & -2.56249200 & 0.62389300 & 0.45351200
\end{array} \\
& \text { C } \quad-3.21859100 \quad 1.82405000 \quad 0.16106600 \\
& \begin{array}{llll}
\text { C } & -4.50106300 & 1.84770900 & -0.40111300
\end{array} \\
& \mathrm{H} \quad-6.14872800 \quad 0.63687800 \quad-1.12326600 \\
& \text { H } \quad-4.98910500 \quad-1.53061100 \quad-0.60655400 \\
& \begin{array}{llll}
\text { C } & -1.18437600 & 0.60818200 & 1.08572400
\end{array} \\
& \text { H } \quad-2.70345600 \quad 2.76745300 \quad 0.37387600
\end{aligned}
$$




$$
\begin{array}{cccc}
\mathrm{H} & -4.98735400 & 2.80220800 & -0.62403400 \\
\mathrm{C} & -0.52761900 & -0.75315200 & 0.96054900 \\
\mathrm{C} & -1.41638600 & -1.87134600 & 0.85941500 \\
\mathrm{H} & -0.99836400 & -2.87183500 & 1.06218600 \\
\mathrm{~N} & -2.65824300 & -1.85593600 & 0.43899200 \\
\mathrm{H} & -0.52606800 & 1.35645700 & 0.60118700 \\
\mathrm{H} & 0.33678900 & -0.91055200 & 1.61456000 \\
\mathrm{H} & -1.26705800 & 0.91590100 & 2.15056100 \\
\mathrm{C} & 4.49576000 & 1.88572900 & 0.18001500 \\
\mathrm{C} & 3.15639300 & 1.82474100 & -0.17043000 \\
\mathrm{C} & 2.48430800 & 0.57607900 & -0.36539200 \\
\mathrm{C} & 3.28608200 & -0.61835000 & -0.21211200 \\
\mathrm{C} & 4.64467100 & -0.52266700 & 0.13922300 \\
\mathrm{C} & 5.26019000 & 0.71051300 & 0.34605200 \\
\mathrm{H} & 4.96812800 & 2.86274200 & 0.33219900 \\
\mathrm{H} & 2.56222700 & 2.73311800 & -0.30161700 \\
\mathrm{C} & 2.62629500 & -1.86874900 & -0.50541200 \\
\mathrm{H} & 5.22186800 & -1.44902100 & 0.24766300 \\
\mathrm{H} & 6.31608200 & 0.76800300 & 0.62556300 \\
\mathrm{C} & 1.30103800 & -1.88235100 & -0.79449000 \\
\mathrm{C} & 0.51775000 & -0.63952300 & -0.74163500 \\
\mathrm{H} & 3.21648800 & -2.79271000 & -0.50976900 \\
\mathrm{H} & 0.78328000 & -2.81880700 & -1.02696700 \\
\mathrm{H} & -0.37923100 & -0.63747700 & -1.37861400 \\
\mathrm{~N} & 1.18436000 & 0.56248800 & -0.72076000
\end{array}
$$

\section{0-ts}

$\begin{array}{llll}\text { C } & -5.71078600 & -0.56603400 & 0.06626400\end{array}$

$$
\begin{array}{llll}
\mathrm{C} & -4.59075500 & -1.39348100 & 0.01961500 \\
\mathrm{C} & -3.28356800 & -0.86950100 & -0.11775800 \\
\mathrm{C} & -3.13427000 & 0.54561200 & -0.18337800 \\
\mathrm{C} & -4.27173900 & 1.35836900 & -0.15939700 \\
\mathrm{C} & -5.56018000 & 0.82348200 & -0.03285200 \\
\mathrm{H} & -6.70883400 & -1.00430700 & 0.17235400 \\
\mathrm{H} & -4.68218800 & -2.48169100 & 0.07330500 \\
\mathrm{C} & -1.73651500 & 1.13968700 & -0.21545400 \\
\mathrm{H} & -4.14343400 & 2.44579300 & -0.22405900 \\
\mathrm{H} & -6.43261800 & 1.48325200 & -0.00349100 \\
\mathrm{C} & -0.67450100 & 0.11383500 & -0.56824500 \\
\mathrm{C} & -1.05333400 & -1.25143000 & -0.54902300 \\
\mathrm{H} & -0.25376800 & -1.97896400 & -0.75547300 \\
\mathrm{~N} & -2.22739100 & -1.76062900 & -0.23422900 \\
\mathrm{H} & -1.53318200 & 1.57797500 & 0.79218100 \\
\mathrm{C} & 5.23004700 & -1.34575600 & -0.17746200 \\
\mathrm{C} & 3.94858000 & -1.57248800 & 0.31606900
\end{array}
$$

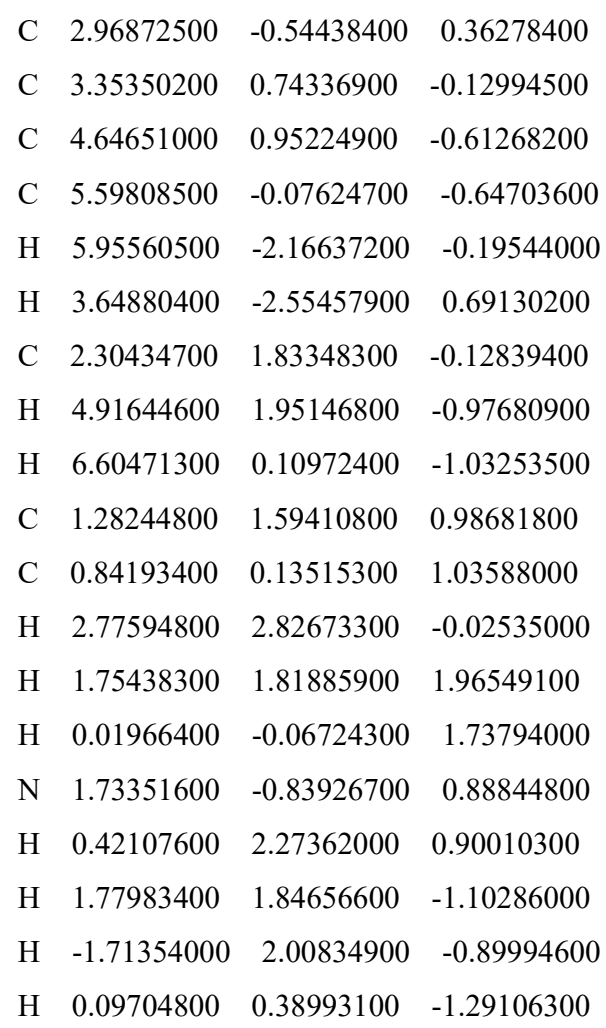




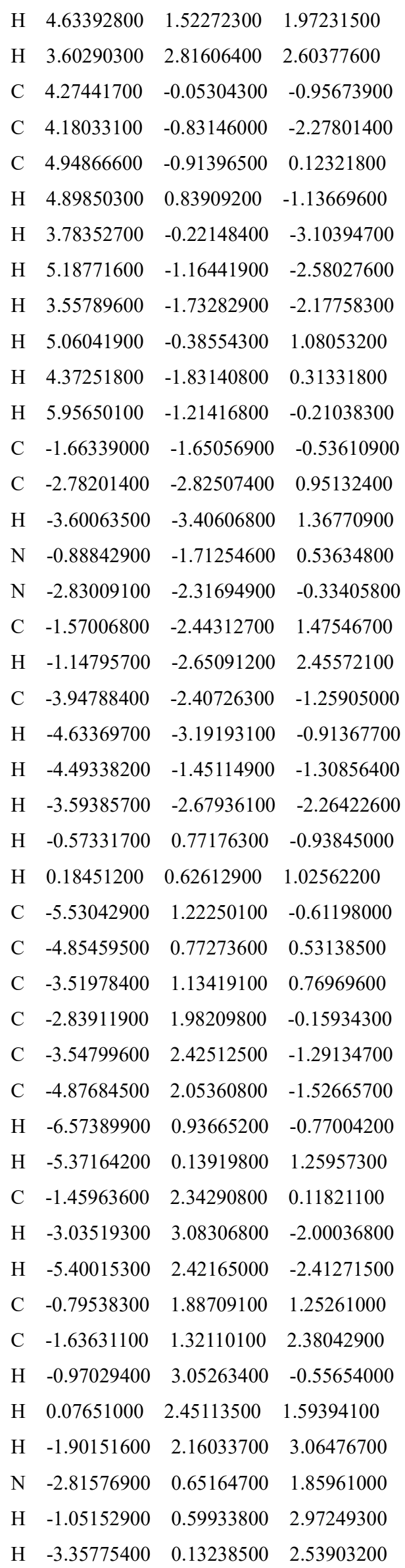

\section{2-ts}

$\begin{array}{llll}\mathrm{P} & -0.13317700 & 2.42624500 & 0.01426200\end{array}$

$\begin{array}{llll}\text { C } & 1.72958800 & 2.60431000 & -0.06525600\end{array}$

$\begin{array}{llll}\text { C } & 2.37978800 & 1.37039300 & 0.54746700\end{array}$

H $\quad 2.08912600 \quad 3.51897500 \quad 0.43155600$

H $2.01834800 \quad 2.68164800 \quad-1.12718200$

H $\quad 3.47218400 \quad 1.40257300 \quad 0.38935600$

$\mathrm{H} \quad 2.19682500 \quad 1.33240400 \quad 1.63256300$

N $\quad 1.80564200 \quad 0.13860000 \quad-0.04220100$

$\begin{array}{llll}\text { C } & 2.53022300 & -1.05529200 & 0.41468000\end{array}$

$\mathrm{H} \quad 1.94157400 \quad-1.95178900 \quad 0.16541000$

$\begin{array}{llll}\mathrm{H} & 2.59831700 & -1.03133800 & 1.51456400\end{array}$

$\begin{array}{llll}\mathrm{Mn} & -0.48461500 & 0.18102500 & 0.07248700\end{array}$

C $\quad-0.41723000 \quad-0.12581400 \quad 1.84944600$

$\begin{array}{llll}\text { O } & -0.35698400 & -0.40387900 & 2.97614200\end{array}$

$\begin{array}{llll}\text { C } & -2.24991700 & 0.43212300 & 0.10501700\end{array}$

O $\quad-3.38161700 \quad 0.67082200 \quad 0.18642300$

$\begin{array}{llll}\text { C } & -0.63117800 & 3.36857200 & -1.55272300\end{array}$

$\begin{array}{llll}\text { C } & -0.27781400 & 4.86170500 & -1.55017200\end{array}$

$\begin{array}{llll}\text { C } & -2.08131700 & 3.13477200 & -1.99602000\end{array}$

$\begin{array}{llll}\mathrm{H} & 0.01303900 & 2.86306800 & -2.29461200\end{array}$

$\mathrm{H} \quad 0.76724800 \quad 5.05211700 \quad-1.25863700$

$\begin{array}{llll}\mathrm{H} & -0.41721200 & 5.28367400 & -2.55983300\end{array}$

H $\quad-0.92855500 \quad 5.43539800 \quad-0.87094100$

$\begin{array}{llll}\mathrm{H} & -2.31753300 & 2.06478400 & -2.07167200\end{array}$

H $\quad-2.80885600 \quad 3.59123500 \quad-1.30769100$

H $\quad-2.24259900 \quad 3.58917400 \quad-2.98845200$

$\begin{array}{llll}\text { C } & -0.59008900 & 3.59380500 & 1.43121200\end{array}$

$\begin{array}{llll}\text { C } & 0.14318400 & 3.24739300 & 2.73475200\end{array}$

C $\quad-2.10596900 \quad 3.64244500 \quad 1.66645300$

$\mathrm{H} \quad-0.25196400 \quad 4.59396400 \quad 1.11002500$

$\mathrm{H} \quad 1.23856400 \quad 3.29261500 \quad 2.63313000$

$\mathrm{H} \quad-0.13598400 \quad 3.97255100 \quad 3.51769100$

$\mathrm{H} \quad-0.12603000 \quad 2.24668100 \quad 3.10315600$

H $\quad-2.66262300 \quad 3.97292000 \quad 0.77782600$

$\mathrm{H} \quad-2.49989900 \quad 2.65688500 \quad 1.95627300$

H $\quad-2.33684400 \quad 4.34858700 \quad 2.48178300$

$\begin{array}{llll}\text { C } & 3.88715300 & -1.17991800 & -0.23017000\end{array}$

$\begin{array}{llll}\text { C } & 5.91355600 & -1.91216300 & -0.70375200\end{array}$

$\begin{array}{llll}\mathrm{H} & 6.83288600 & -2.48290800 & -0.59611200\end{array}$

N $4.23929000 \quad-0.54318500 \quad-1.32898300$

N $4.87146900 \quad-2.02993300 \quad 0.19902400$

$\begin{array}{llll}\text { C } & 5.50131700 & -0.98743100 & -1.63385600\end{array}$

H $\quad 6.04864200 \quad-0.61926000 \quad-2.50049300$ 


$$
\begin{array}{llll}
\mathrm{C} & 4.81634000 & -2.92018000 & 1.34466300 \\
\mathrm{H} & 5.80379200 & -3.37535100 & 1.49523600 \\
\mathrm{H} & 4.54846000 & -2.36878200 & 2.25884200 \\
\mathrm{H} & 4.08036200 & -3.72618200 & 1.19271300 \\
\mathrm{H} & 1.97947700 & 0.19578900 & -1.05008400 \\
\mathrm{H} & -0.38058500 & 0.36144300 & -1.49974600 \\
\mathrm{C} & -4.47176700 & -3.83015600 & 0.47191900 \\
\mathrm{C} & -4.24796300 & -3.05913600 & -0.67111000 \\
\mathrm{C} & -3.00128000 & -2.45378600 & -0.89347900 \\
\mathrm{C} & -1.95664000 & -2.62419400 & 0.05359500 \\
\mathrm{C} & -2.20389600 & -3.41363100 & 1.18505200 \\
\mathrm{C} & -3.44627900 & -4.01633400 & 1.40373200 \\
\mathrm{H} & -5.45082600 & -4.29083500 & 0.62861100 \\
\mathrm{H} & -5.04830200 & -2.91816500 & -1.40433700 \\
\mathrm{C} & -0.65309800 & -1.97041500 & -0.18717600 \\
\mathrm{H} & -1.40081800 & -3.54243200 & 1.91716000 \\
\mathrm{H} & -3.61287800 & -4.62291300 & 2.29721300 \\
\mathrm{C} & -0.39806000 & -1.34013700 & -1.44074300 \\
\mathrm{C} & -1.42558300 & -1.55553900 & -2.54269100 \\
\mathrm{H} & 0.17386600 & -2.43033600 & 0.35846200 \\
\mathrm{H} & 0.62378500 & -1.41854000 & -1.83359800 \\
\mathrm{H} & -1.13503800 & -2.48844500 & -3.08108300 \\
\mathrm{~N} & -2.76718300 & -1.64043400 & -1.99936100 \\
\mathrm{H} & -1.39471000 & -0.73822500 & -3.28073300 \\
\hline & -3.50823100 & -1.64138200 & -2.69062000 \\
\hline & & & \\
\end{array}
$$

\section{3-ts}

$\begin{array}{llll}\mathrm{P} & 2.25046400 & 0.33144800 & -0.28915800\end{array}$

$\begin{array}{llll}\mathrm{C} & 1.42205700 & 1.27921400 & -1.67741900 \\ \mathrm{C} & 0.28502500 & 0.45104000 & -2.28590400 \\ \mathrm{H} & 2.13360600 & 1.60550000 & -2.45142000 \\ \mathrm{H} & 1.00254400 & 2.18700800 & -1.21748900 \\ \mathrm{H} & -0.36463200 & 1.12186800 & -2.88590800 \\ \mathrm{H} & 0.70183800 & -0.29305100 & -2.99795400 \\ \mathrm{~N} & -0.47282600 & -0.23048300 & -1.25044100 \\ \mathrm{C} & -1.58920100 & -1.00453400 & -1.74853900 \\ \mathrm{H} & -1.29236800 & -1.61628300 & -2.63160500 \\ \mathrm{H} & -2.44090200 & -0.37120300 & -2.07662000 \\ \mathrm{Mn} & 0.51191400 & -1.04210700 & 0.39018100 \\ \mathrm{C} & 1.27197900 & -2.49494700 & -0.26657700 \\ \mathrm{O} & 1.70034600 & -3.48822900 & -0.69398300 \\ \mathrm{C} & 1.09409100 & -1.40773200 & 2.04658000 \\ \mathrm{O} & 1.46240800 & -1.64019700 & 3.12261500 \\ \mathrm{C} & 2.88481100 & 1.73637400 & 0.80738000 \\ \mathrm{C} & 3.70536800 & 2.80897100 & 0.07909700\end{array}$

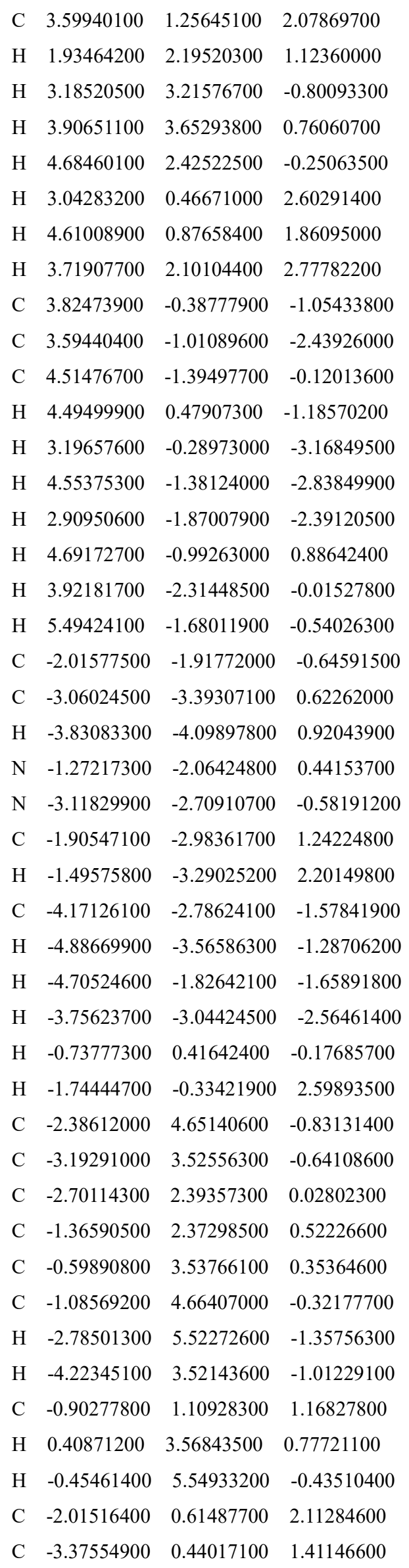




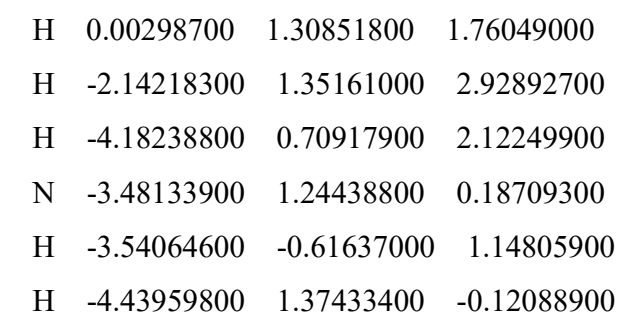

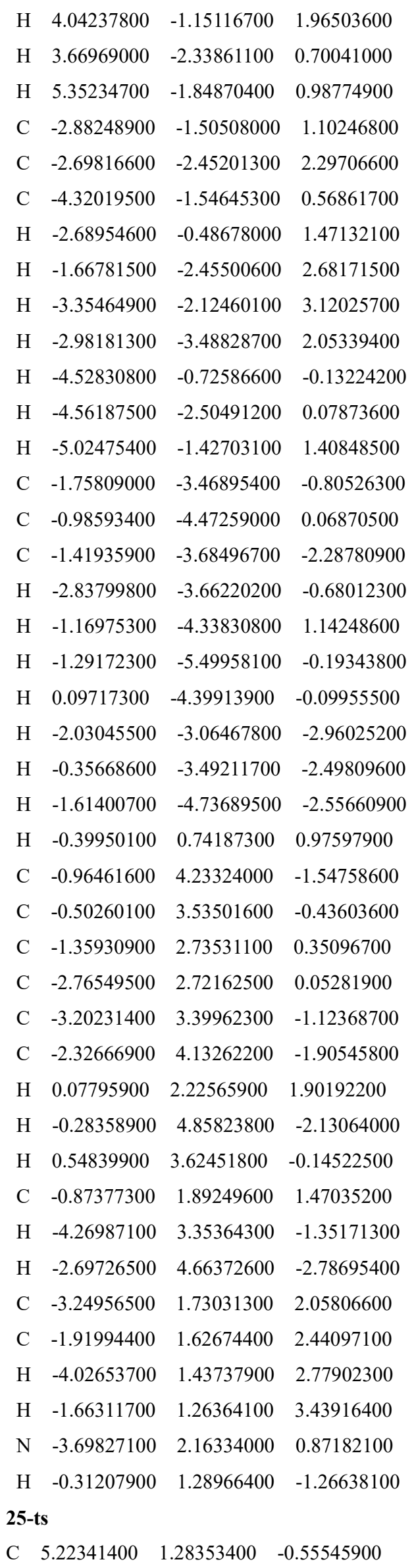




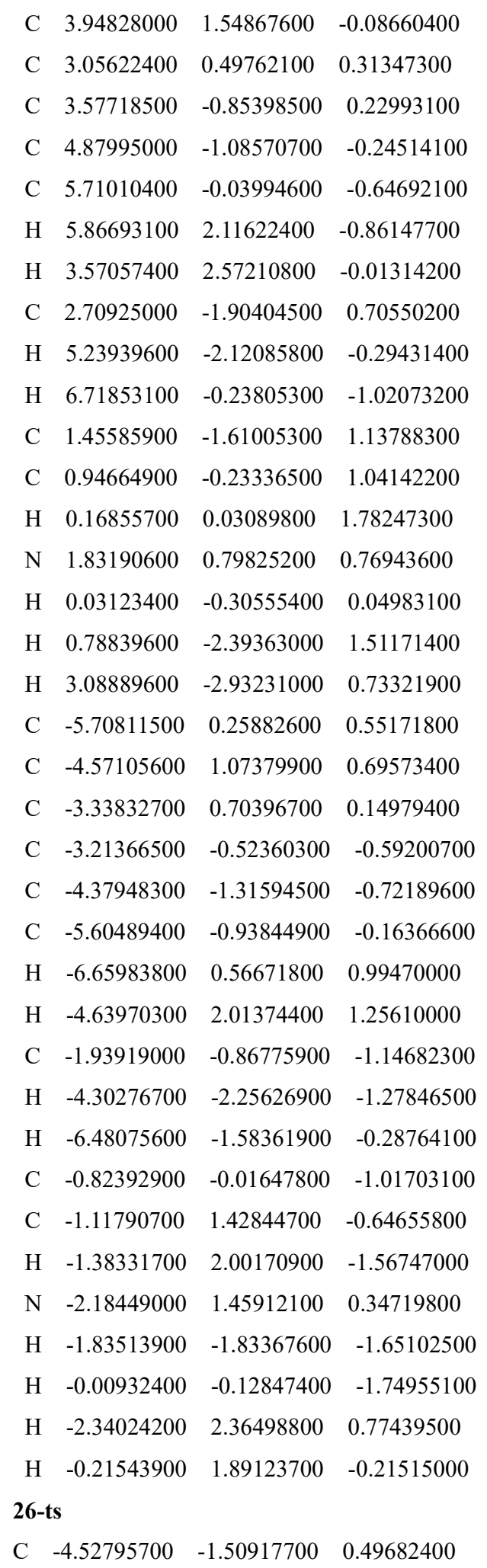

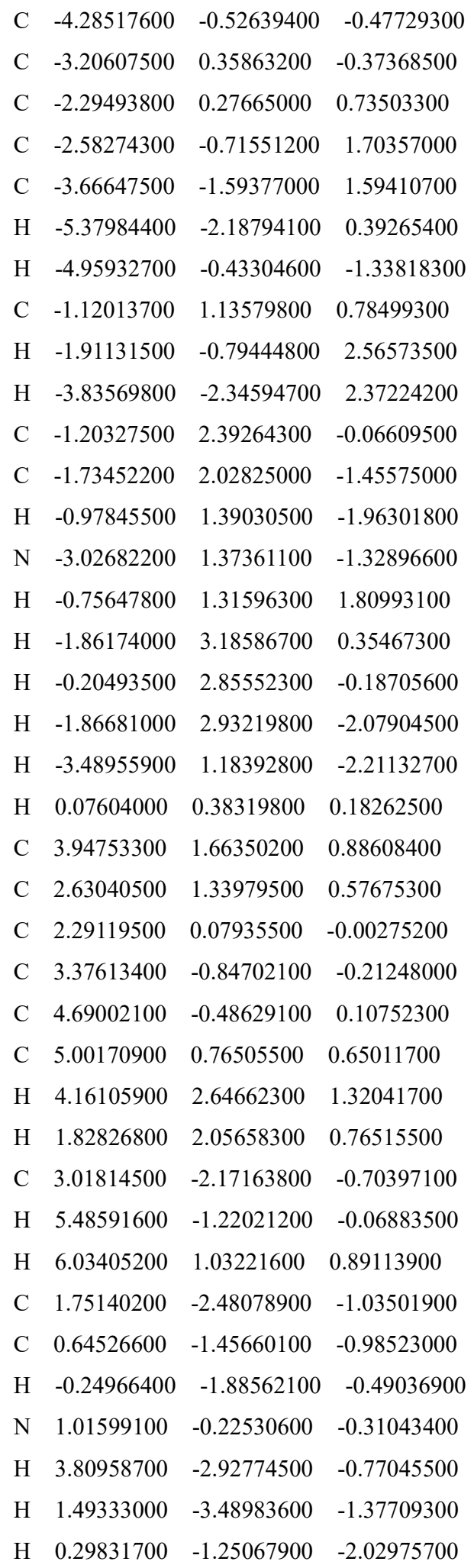




\section{Reference}

(1) Joseph, P. J. A.; Priyadarshini, S.; Kantam, M. L.; Sreedhar, B. Tetrahedron 2013, 69, 8276.

(2) Adam, R.; Cabrero-Antonino, J. R.; Spannenberg, A.; Junge, K.; Jackstell, R.; Beller, M. Angew. Chem. Int. Ed. 2017, 56, 3216.

(3) Pi, D.; Jiang, K.; Zhou, H.; Sui, Y.; Uozumi, Y.; Zou, K. RSC Adv. 2014, 4, 57875.

(4) Fu, S.; Shao, Z.; Wang, Y.; Liu, Q. J. Am. Chem. Soc. 2017, 139, 11941.

(5) Zubar, V.; Lebedev, Y.; Azofra, L. M.; Cavallo, L.; El-Sepelgy, O.; Rueping, M. Angew. Chem. Int. Ed. 2018, 57, 13439.

(6) Jang, Y. K.; Krückel, T.; Rueping, M.; El-Sepelgy, O. Org. Lett. 2018, 20, 7779.

(7) Toutov, A. A.; Betz, K. N.; Schuman, D. P.; Liu, W.-B.; Fedorov, A.; Stoltz, B. M.; Grubbs, R. H. J. Am. Chem. Soc. 2017, 139, 1668.

(8) Zhong, R.; Wei, Z.; Zhang, W.; Liu, S.; Liu, Q. Chem 2019, 5, 1552.

(9) Barrios, F. J.; Springer, B. C.; Colby, D. A. Org. Lett. 2013, 15, 3082.

(10) Ren, T.-B.; Xu, W.; Zhang, W.; Zhang, X.-X.; Wang, Z.-Y.; Xiang, Z.; Yuan, L.; Zhang, X.-B. J. Am. Chem. Soc. 2018, 140, 7716 .

(11) Forrest, T. P.; Dauphinee, G. A.; Deraniyagala, S. A. Can. J. Chem. 1985, 63, 412.

(12) (a) Mani, N. S.; Chen, P.; Jones, T. K. J. Org. Chem. 1999, 64, 6911; (b) Erős, G.; Nagy, K.; Mehdi, H.; Pápai, I.; Nagy, P.; Király, P.; Tárkányi, G.; Soós, T. Chem. Eur. J. 2012, 18, 574; (c) Talwar, D.; Li, H. Y.; Durham, E.; Xiao, J. Chem. Eur. J. 2015, 21, 5370.

(13) (a) Chen, X.; Zhao, H.; Chen, C.; Jiang, H.; Zhang, M. Org. Lett. 2018, 20, 1171; (b) Chen, X.; Zhao, H.; Chen, C.; Jiang, H.; Zhang, M. Chem. Commun. 2018, 54, 9087.

(14) Symeonidis, T. S.; Lykakis, I. N.; Litinas, K. E. Tetrahedron 2013, 69, 4612.

(15) Kuriyama, M.; Matsuo, S.; Shinozawa, M.; Onomura, O. Org. Lett. 2013, 15, 2716.

(16) Kuwano, R.; Ikeda, R.; Hirasada, K. Chem. Commun. 2015, 51, 7558.

(17) Marchand, P.; Puget, A.; Baut, G. L.; Emig, P.; Czech, M.; Günther, E. Tetrahedron 2005, 61, 4035.

(18) Xu, Y.; Cong, T.; Liu, P.; Sun, P. Org. Biomol. Chem. 2015, 13, 9742.

(19) Iosub, A. V.; Stahl, S. S. Org. Lett. 2015, 17, 4404.

(20) Yang, C.-H.; Chen, X.; Li, H.; Wei, W.; Yang, Z.; Chang, J. Chem. Commun. 2018, 54, 8622.

(21) Chen, F.; Surkus, A.-E.; He, L.; Pohl, M.-M.; Radnik, J.; Topf, C.; Junge, K.; Beller, M. J. Am. Chem. Soc. 2015, 137,11718

(22) Ferles, M.; Kocian, O.; Lövy, J. Collect. Czech. Chem. Commun. 1976, 41, 758.

(23) Du, Y.-L.; Hu, Y.; Zhu, Y.-F.; Tu, X.-F.; Han, Z.-Y.; Gong, L.-Z. J. Org. Chem. 2015, 80, 4754.

(24) Xiong, B.; Li, Y.; Lv, W.; Tan, Z.; Jiang, H.; Zhang, M. Org. Lett. 2015, 17, 4054.

(25) Xu, C.; Zhang, L.; Dong, C.; Xu, J.; Pan, Y.; Li, Y.; Zhang, H.; Li, H.; Yu, Z.; Xu, L. Adv. Synth. Catal. 2016, $358,567$.

(26) Wu, J.; Barnard, J. H.; Zhang, Y.; Talwar, D.; Robertson, C. M.; Xiao, J. Chem. Commun. 2013, 49, 7052.

(27) Li, S.; Meng, W.; Du, H. Org. Lett. 2017, 19, 2604.

(25). a) A. D. Becke, J. Chem. Phys. 1993, 98, 5648; b) C. Lee, W. Yang, R. G. Parr, Phys. Rev. B 1988, $37,785$.

(26). Zhao, Y.; Truhlar, D. G. Theor. Chem. Acc. 2008, 120, 215

(27). a) A. V. Marenich, C. J. Cramer, D. G. Truhlar, J. Phys. Chem. B 2009, 113, 6378; b) Zhao, Y.; Truhlar, D. G. J. Chem. Phys. 2006, 125, 194101. 


\section{NMR Spectra}

${ }^{1} \mathrm{H}$ NMR $\left(400 \mathrm{MHz}, \mathrm{CDCl}_{3}\right)$ spectrum of $\mathbf{4 a}$
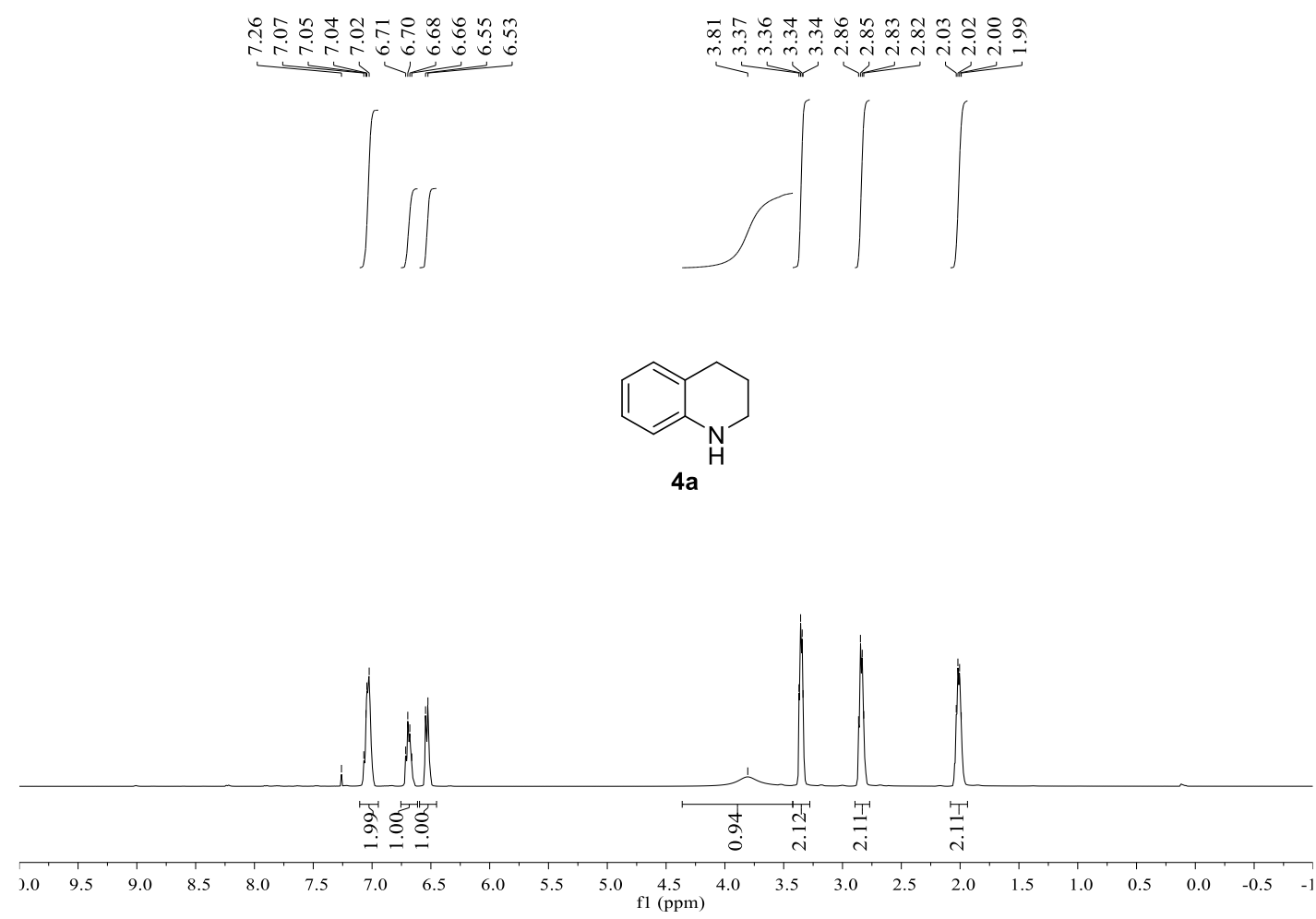

${ }^{13} \mathrm{C}\left\{{ }^{1} \mathrm{H}\right\}$ NMR $\left(101 \mathrm{MHz}, \mathrm{CDCl}_{3}\right)$ spectrum of $\mathbf{4 a}$

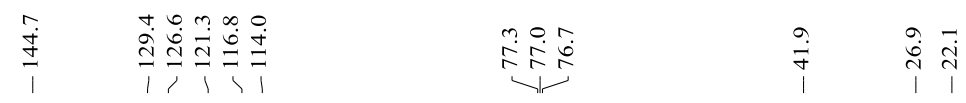<smiles>c1ccc2c(c1)CCCN2</smiles>

4a

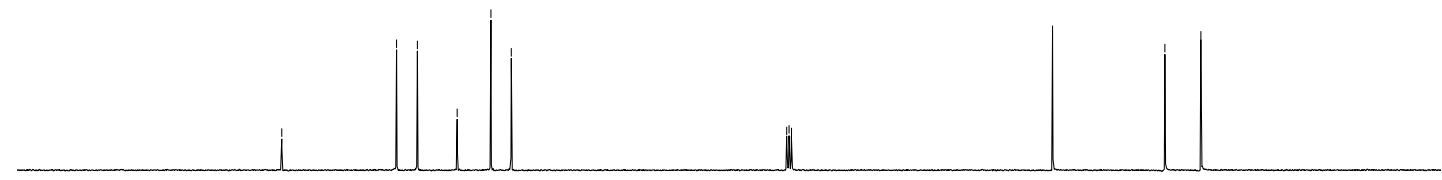

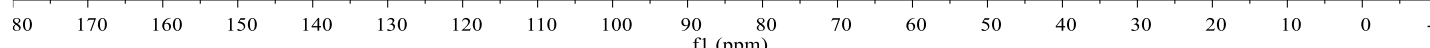


${ }^{1} \mathrm{H}$ NMR $\left(400 \mathrm{MHz}, \mathrm{CDCl}_{3}\right)$ spectrum of $\mathbf{4 b}$

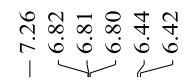

ڤัต

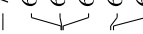

$-7-7-7$
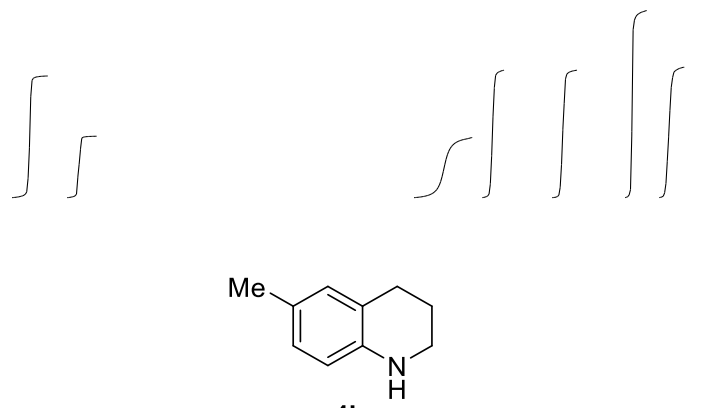

$\mathbf{4 b}$

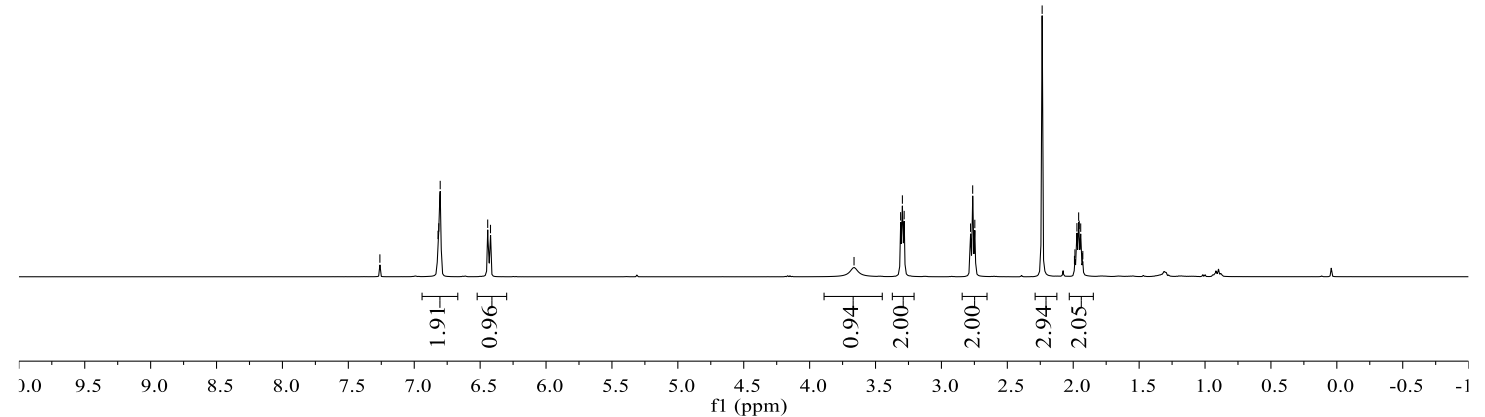

${ }^{13} \mathrm{C}\left\{{ }^{1} \mathrm{H}\right\}$ NMR (101 MHz, $\left.\mathrm{CDCl}_{3}\right)$ spectrum of $\mathbf{4 b}$

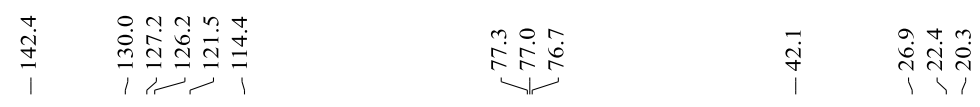<smiles>Cc1ccc2c(c1)CCCN2</smiles>

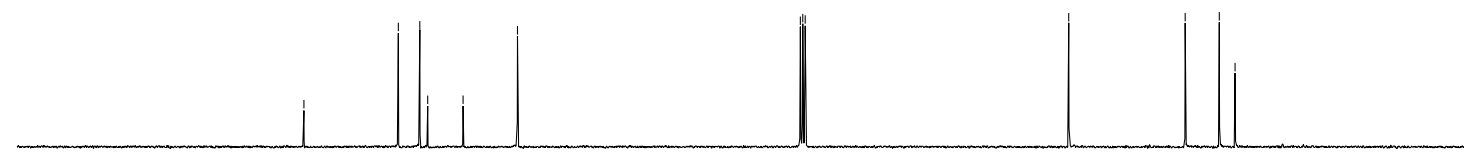

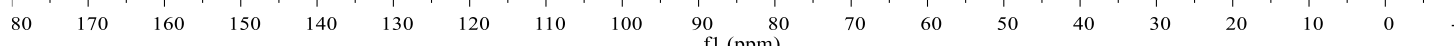


${ }^{1} \mathrm{H}$ NMR (400 MHz, $\left.\mathrm{CDCl}_{3}\right)$ spectrum of $\mathbf{4 c}$

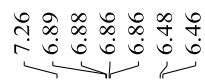

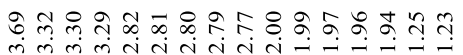

$\int \Gamma$

-<smiles>CC(C)c1ccc2c(c1)CCCN2</smiles>

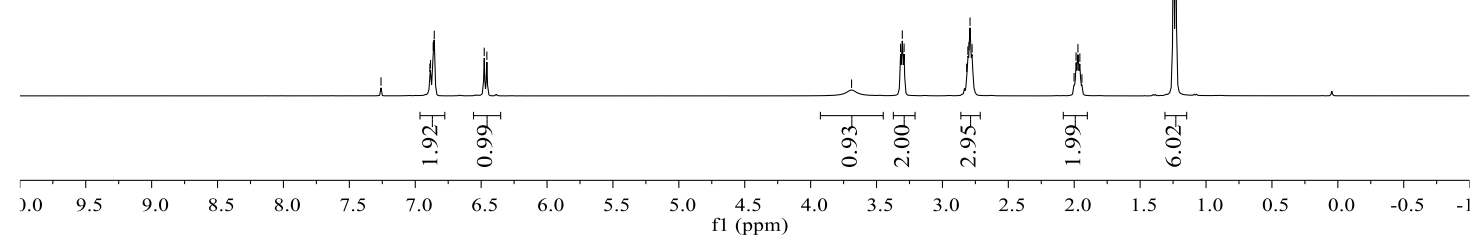

${ }^{13} \mathrm{C}\left\{{ }^{1} \mathrm{H}\right\}$ NMR (101 MHz, $\left.\mathrm{CDCl}_{3}\right)$ spectrum of $\mathbf{4 c}$

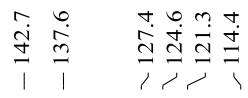

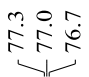

i m ले
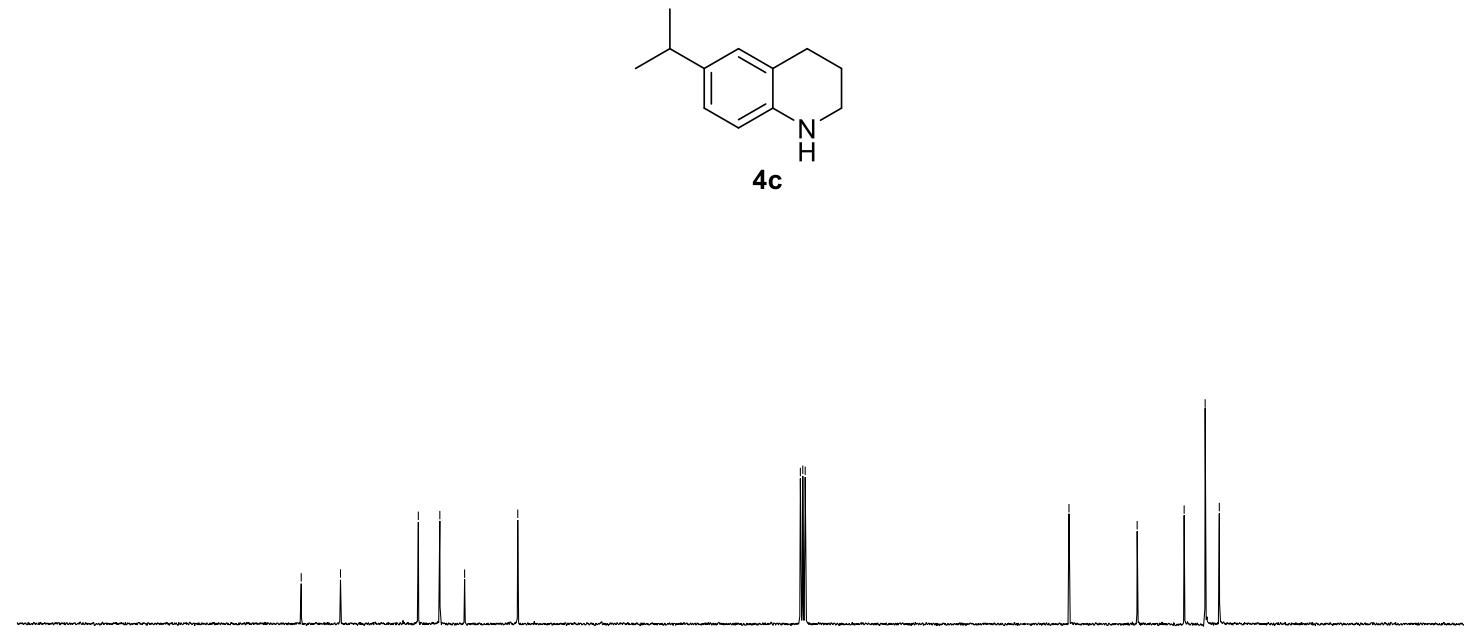

$\begin{array}{llllllllll}80 & 170 & 160 & 150 & 140 & 130 & 120 & 110 & 100 & 90 \\ \mathrm{f} 1(\mathrm{ppm})\end{array}$ 
${ }^{1} \mathrm{H}$ NMR $\left(400 \mathrm{MHz}, \mathrm{CDCl}_{3}\right.$ ) spectrum of $\mathbf{4 d}$
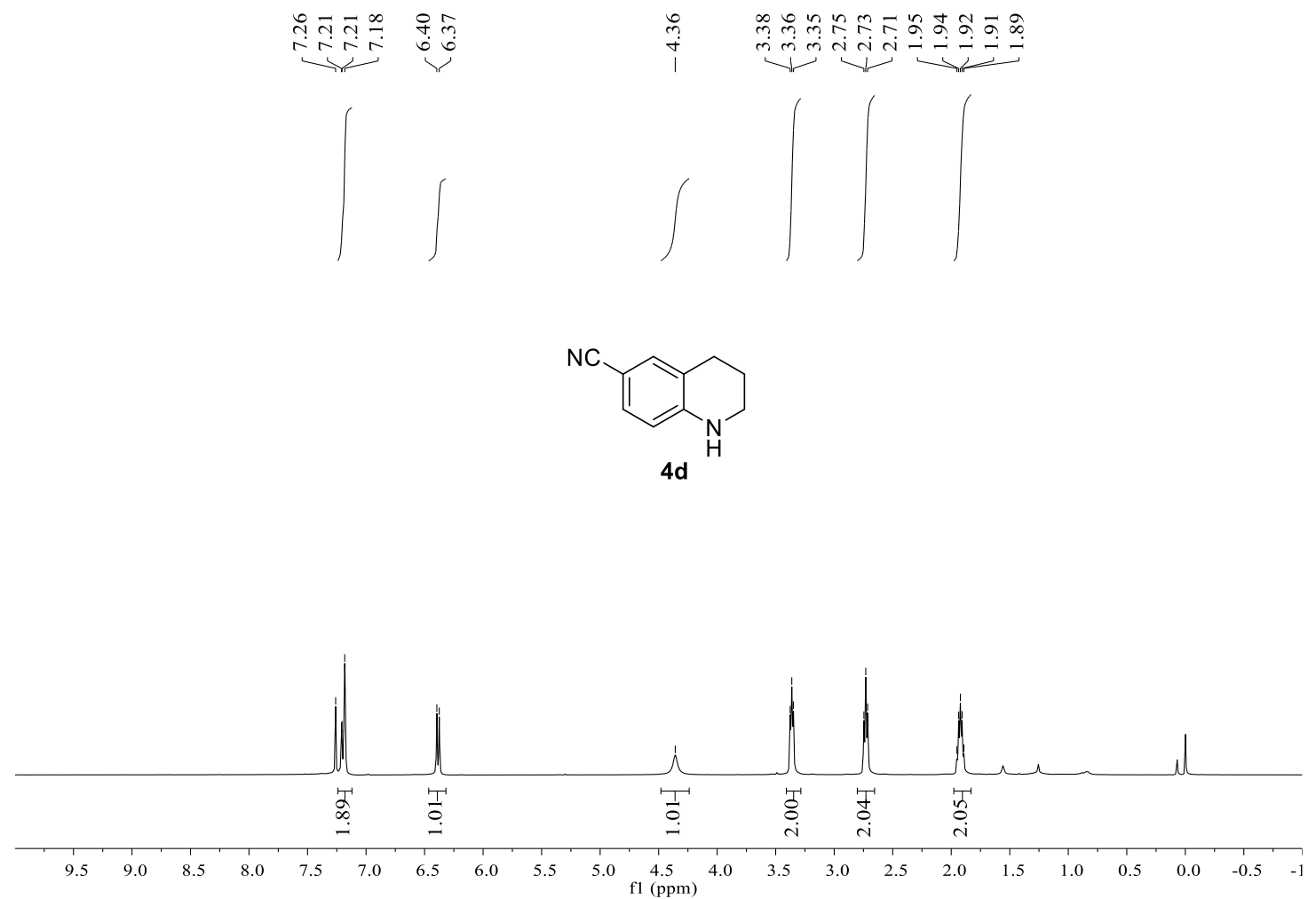

${ }^{13} \mathrm{C}\left\{{ }^{1} \mathrm{H}\right\}$ NMR $\left(101 \mathrm{MHz}, \mathrm{CDCl}_{3}\right)$ spectrum of $\mathbf{4 d}$
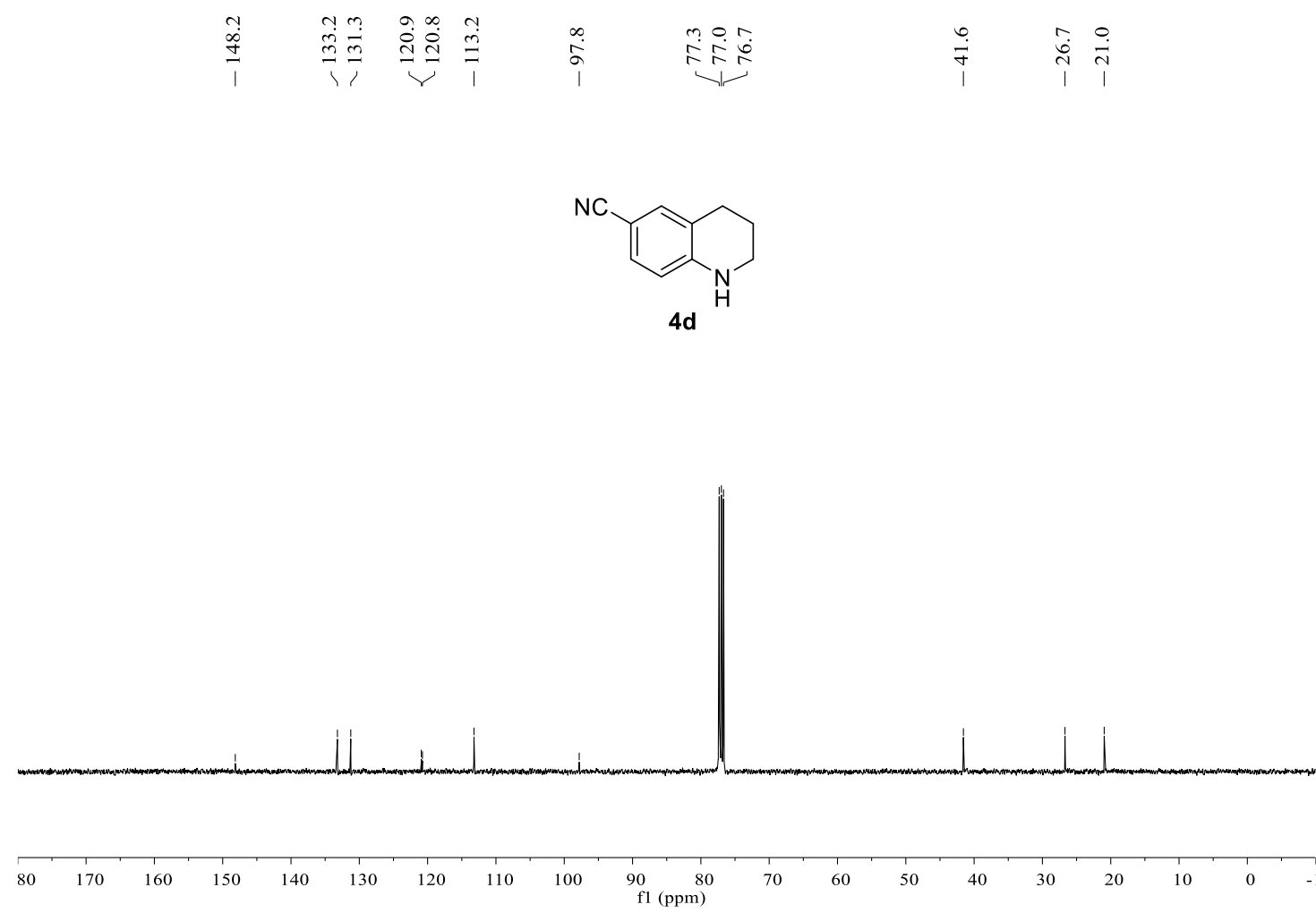
${ }^{1} \mathrm{H}$ NMR $\left(400 \mathrm{MHz}, \mathrm{CDCl}_{3}\right)$ spectrum of $\mathbf{4 e}$
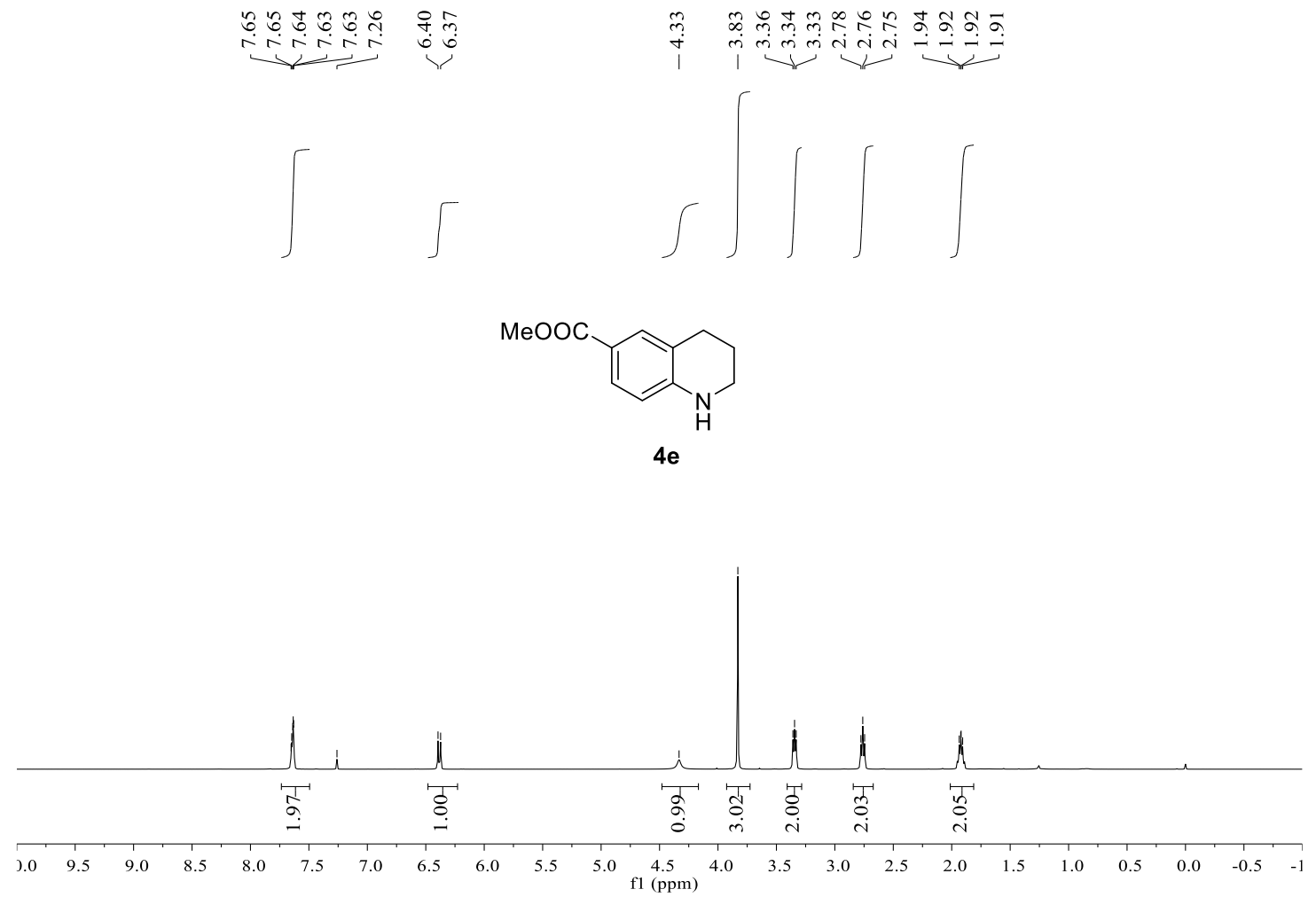

${ }^{13} \mathrm{C}\left\{{ }^{1} \mathrm{H}\right\}$ NMR $\left(101 \mathrm{MHz}, \mathrm{CDCl}_{3}\right)$ spectrum of $\mathbf{4 e}$

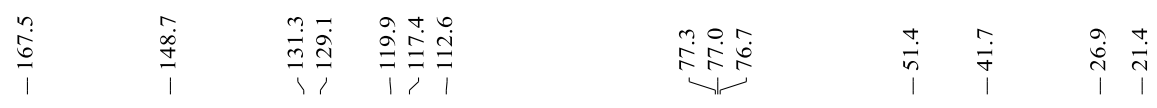
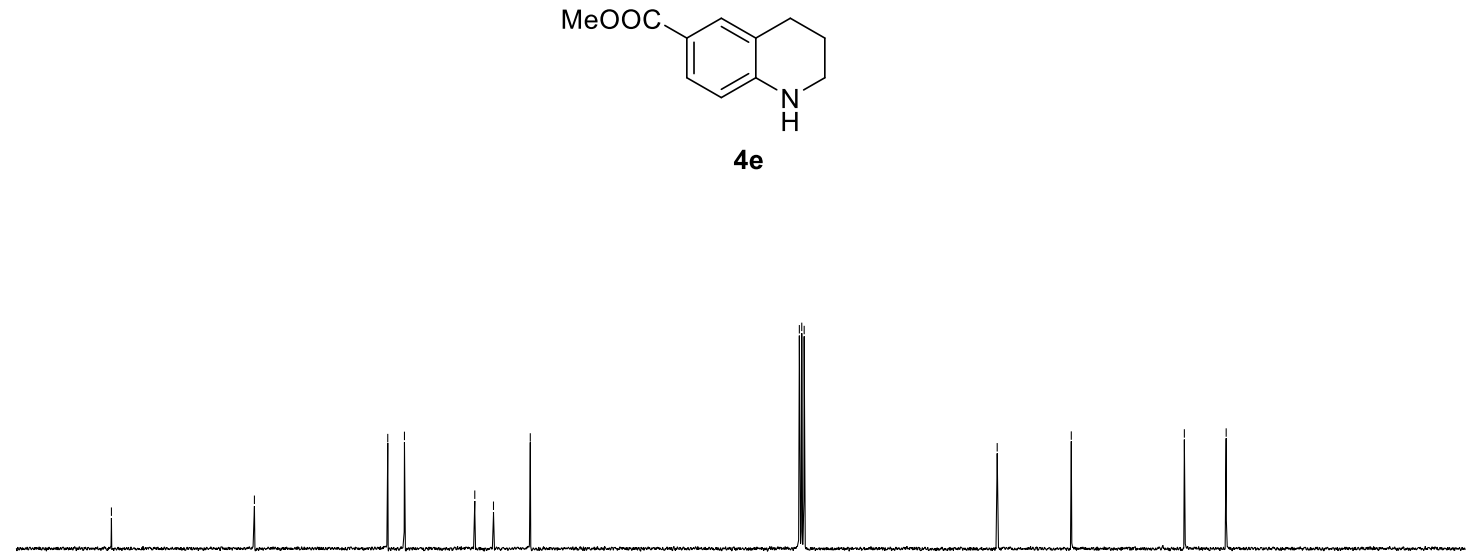

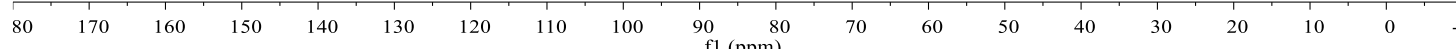


${ }^{1} \mathrm{H}$ NMR $\left(400 \mathrm{MHz}, \mathrm{CDCl}_{3}\right)$ spectrum of $\mathbf{4 f}$

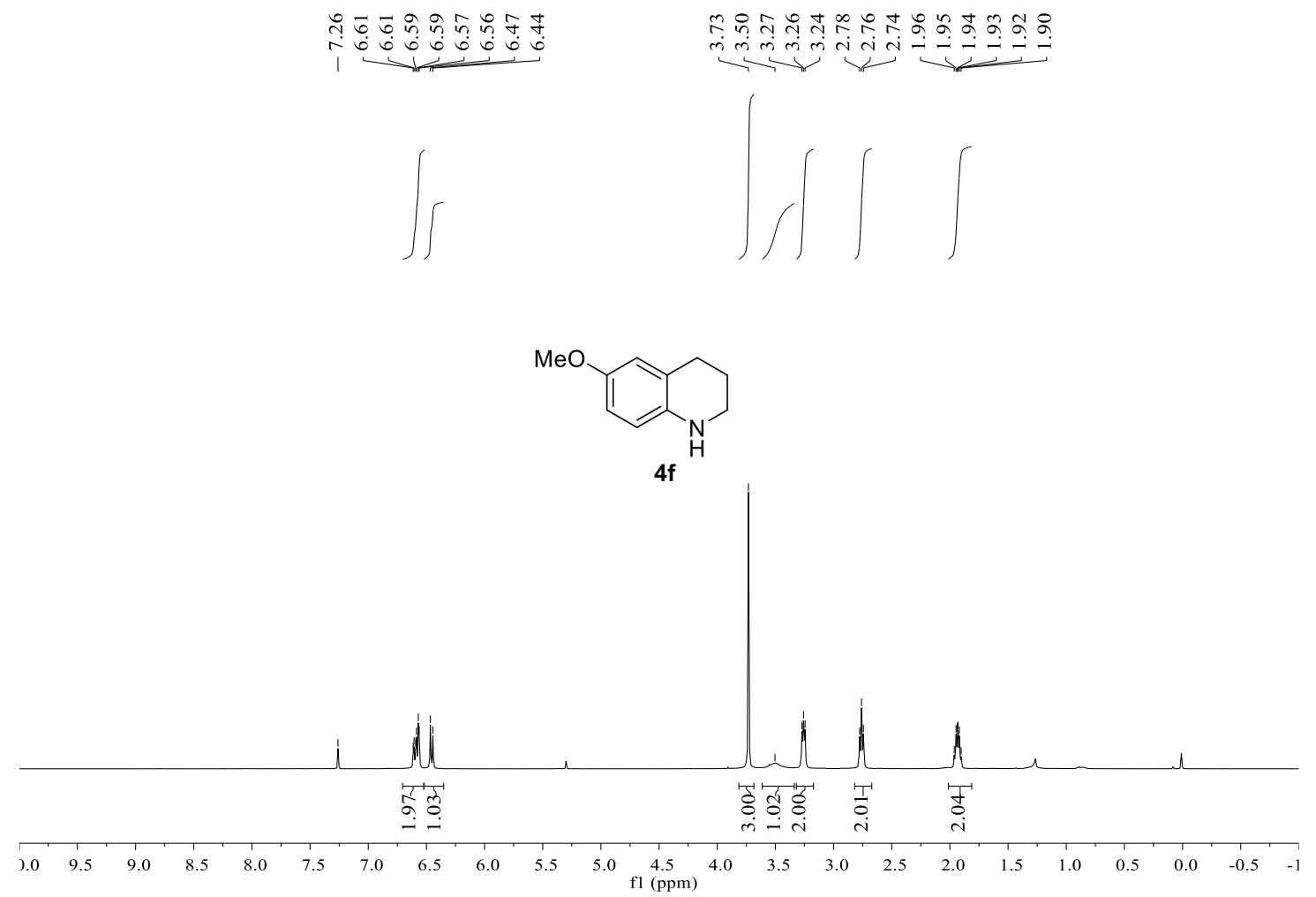

${ }^{13} \mathrm{C}\left\{{ }^{1} \mathrm{H}\right\}$ NMR $\left(101 \mathrm{MHz}, \mathrm{CDCl}_{3}\right)$ spectrum of $\mathbf{4 f}$

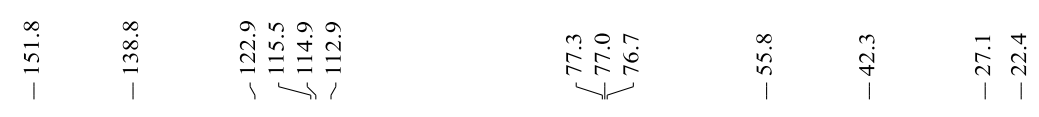
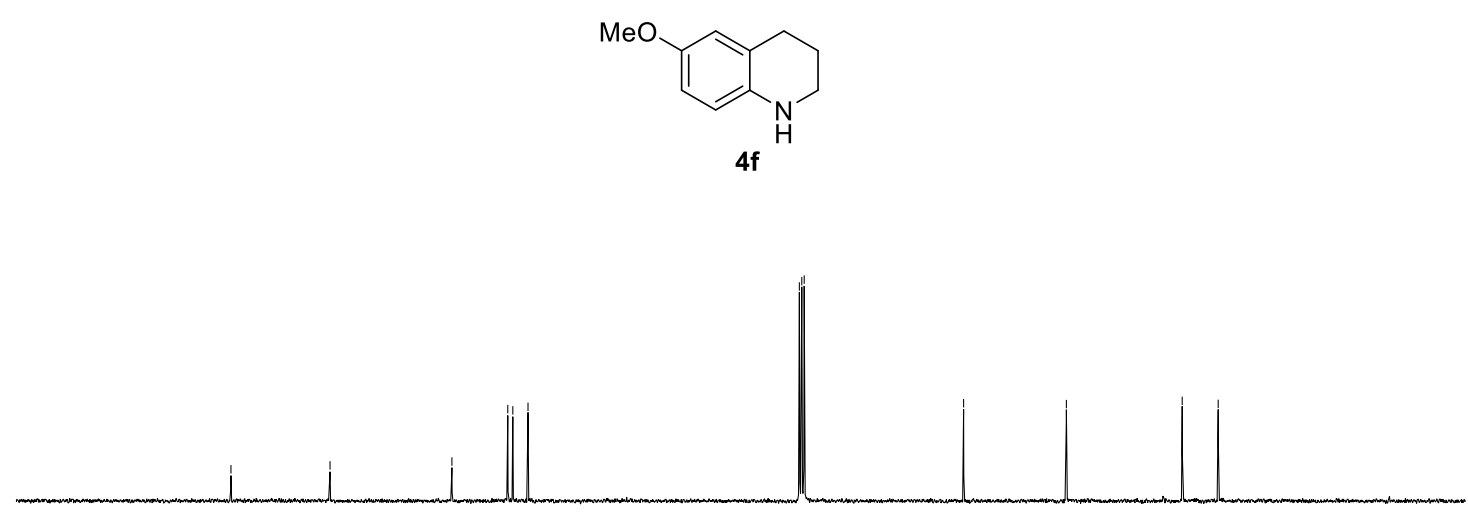

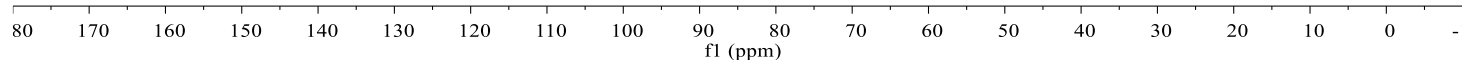


${ }^{1} \mathrm{H}$ NMR $\left(400 \mathrm{MHz}, \mathrm{CDCl}_{3}\right)$ spectrum of $\mathbf{4 g}$

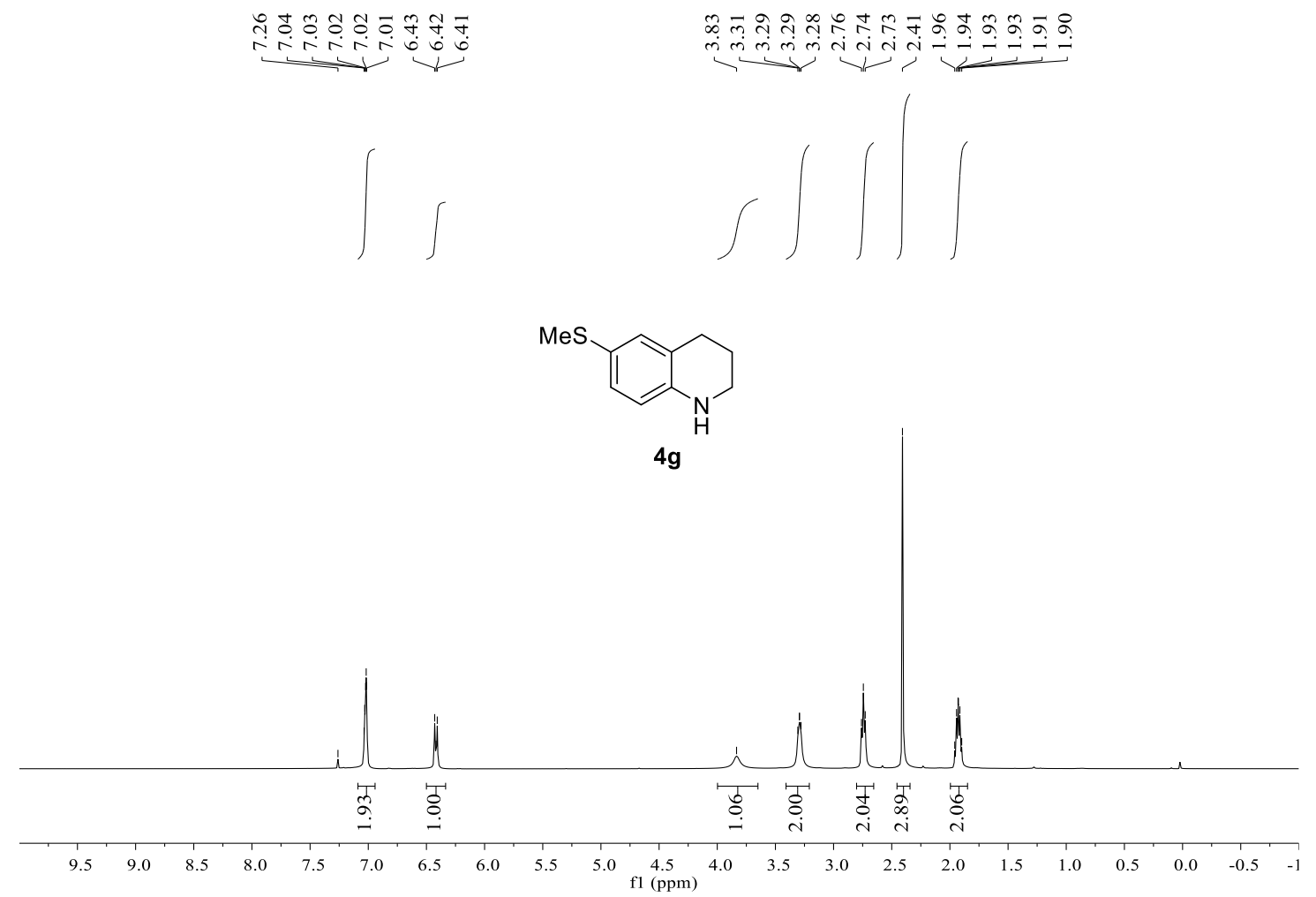

${ }^{13} \mathrm{C}\left\{{ }^{1} \mathrm{H}\right\}$ NMR $\left(101 \mathrm{MHz}, \mathrm{CDCl}_{3}\right)$ spectrum of $\mathbf{4 g}$

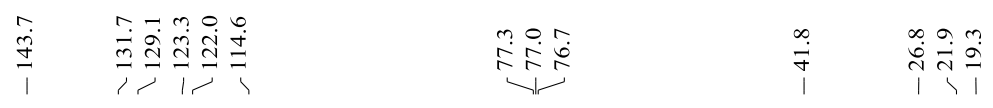
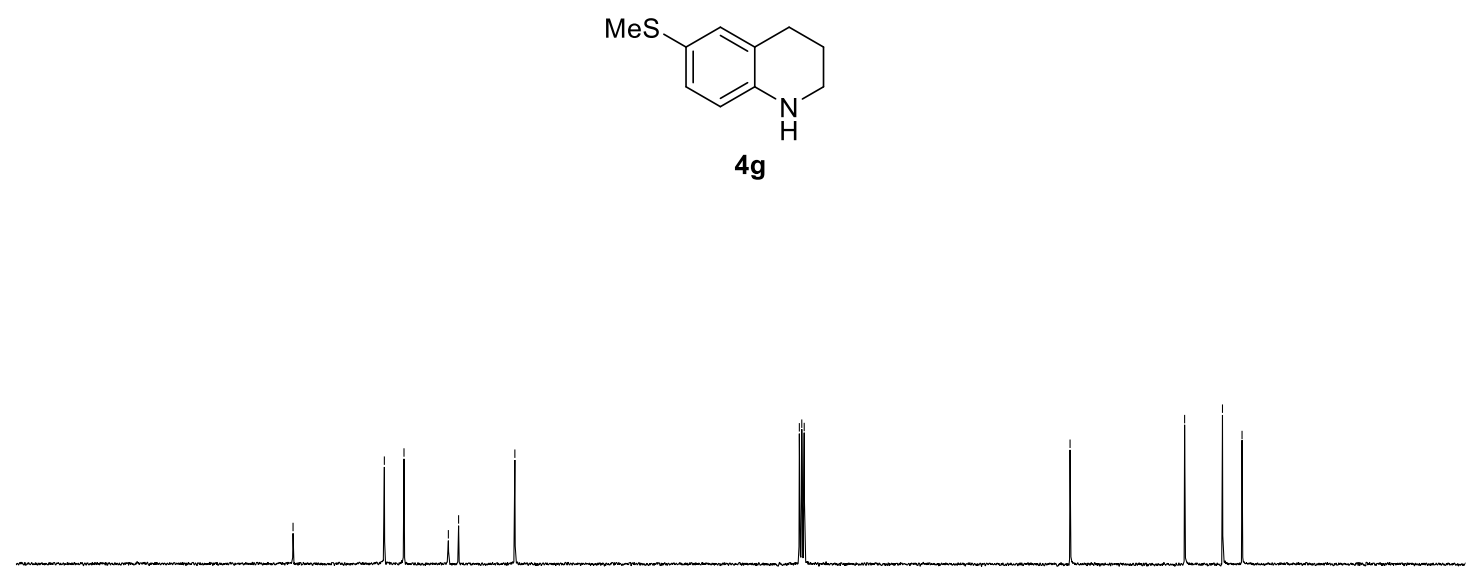

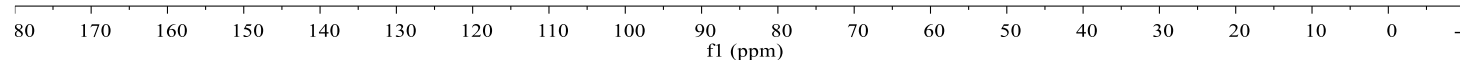


${ }^{1} \mathrm{H}$ NMR $\left(400 \mathrm{MHz}, \mathrm{CDCl}_{3}\right)$ spectrum of $\mathbf{4 h}$
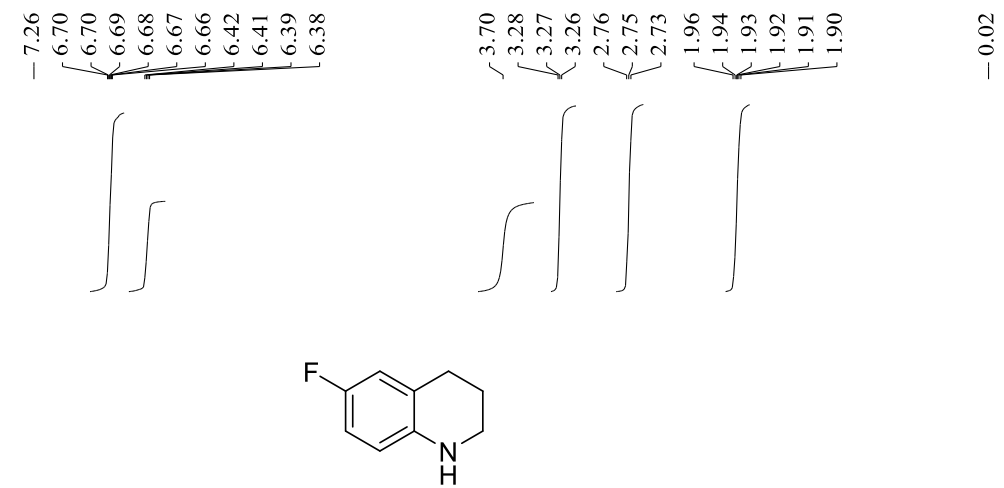

4h

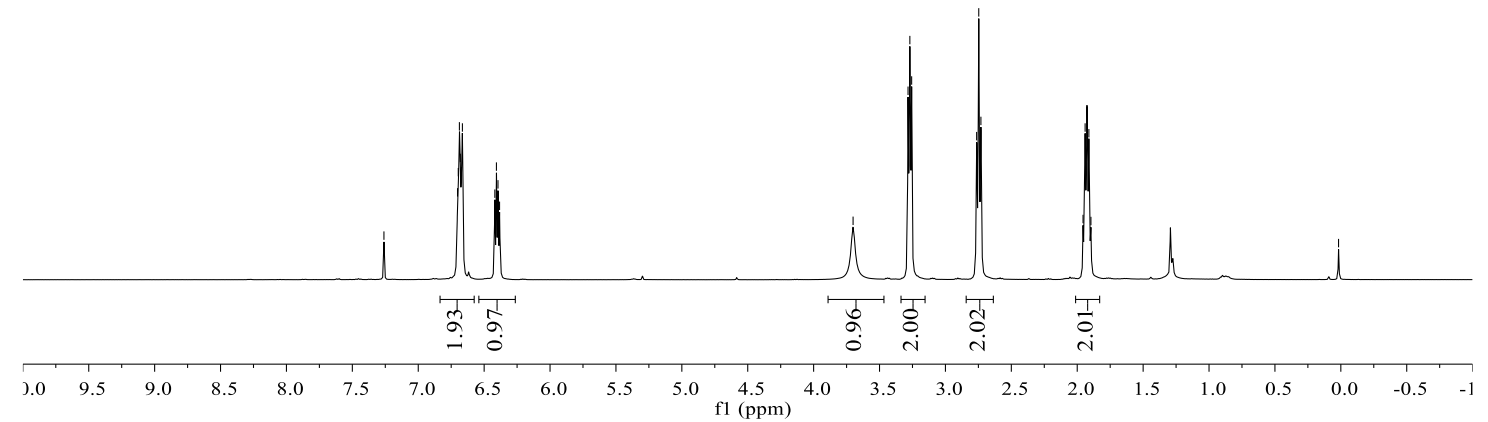

${ }^{13} \mathrm{C}\left\{{ }^{1} \mathrm{H}\right\} \mathrm{NMR}\left(101 \mathrm{MHz}, \mathrm{CDCl}_{3}\right)$ spectrum of $\mathbf{4 h}$

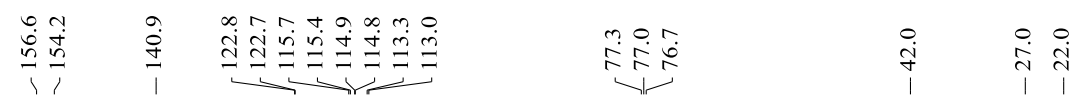

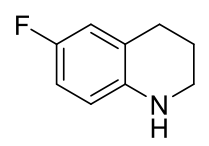

4h

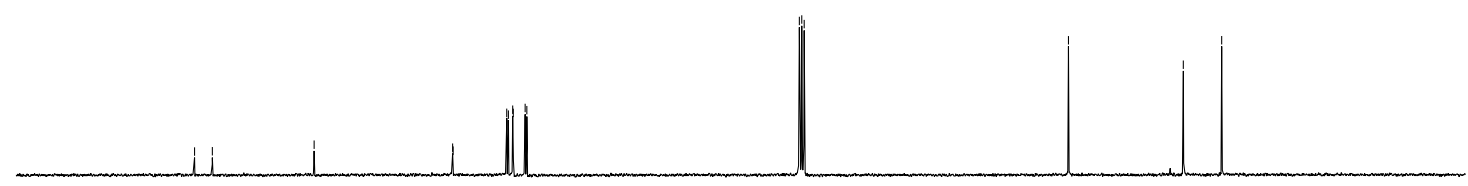

$\begin{array}{llllllllllllllllllll}80 & 170 & 160 & 150 & 140 & 130 & 120 & 110 & 100 & 90 & 80 & 70 & 60 & 50 & 40 & 30 & 20 & 10 & 0 & -\end{array}$ 
${ }^{1} \mathrm{H}$ NMR $\left(400 \mathrm{MHz}, \mathrm{CDCl}_{3}\right)$ spectrum of $\mathbf{4 i}$
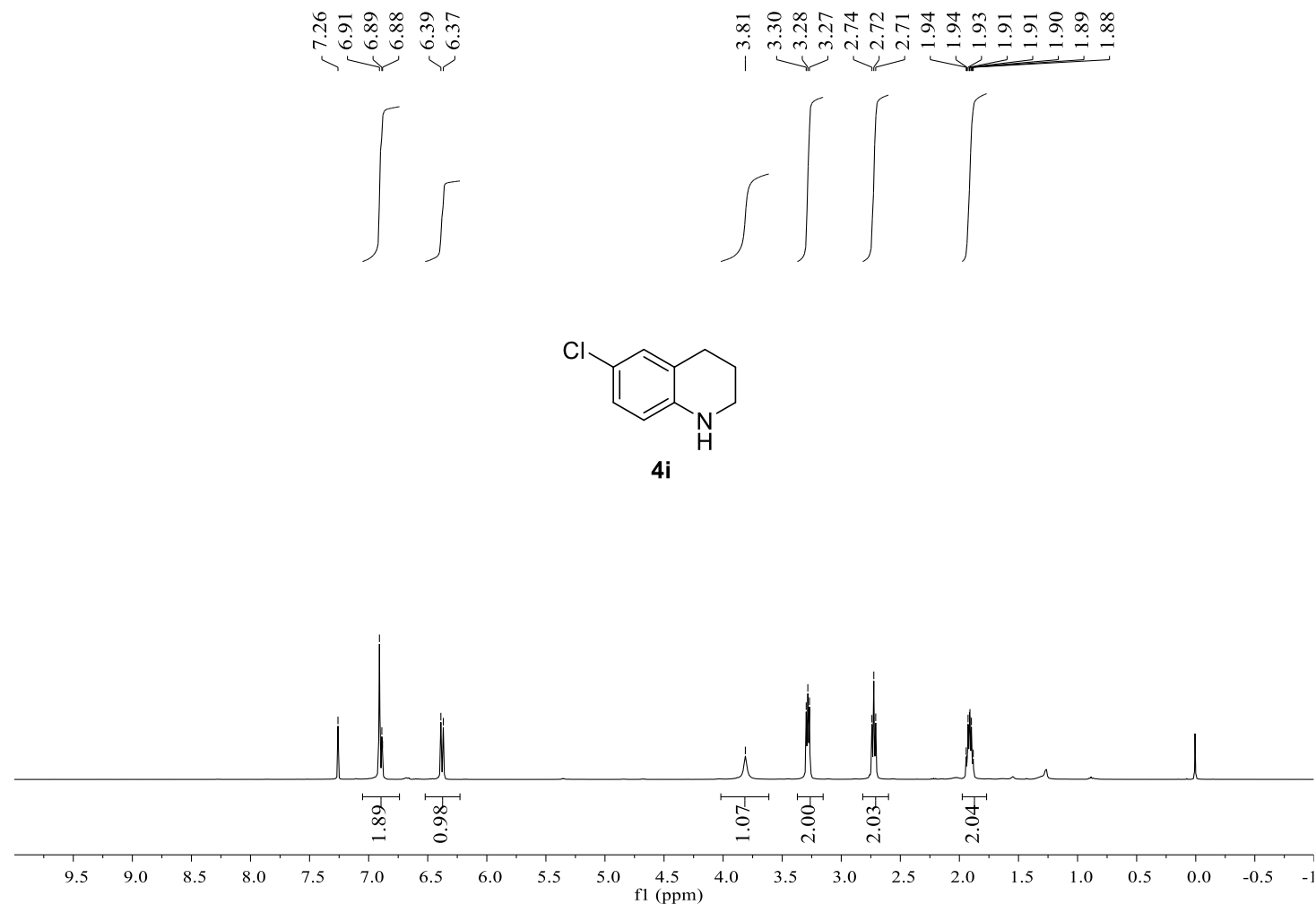

${ }^{13} \mathrm{C}\left\{{ }^{1} \mathrm{H}\right\}$ NMR $\left(101 \mathrm{MHz}, \mathrm{CDCl}_{3}\right)$ spectrum of $\mathbf{4 i}$

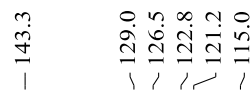

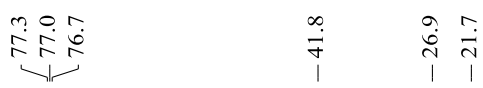

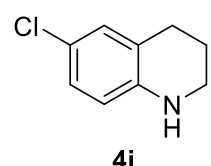

$4 \mathbf{i}$

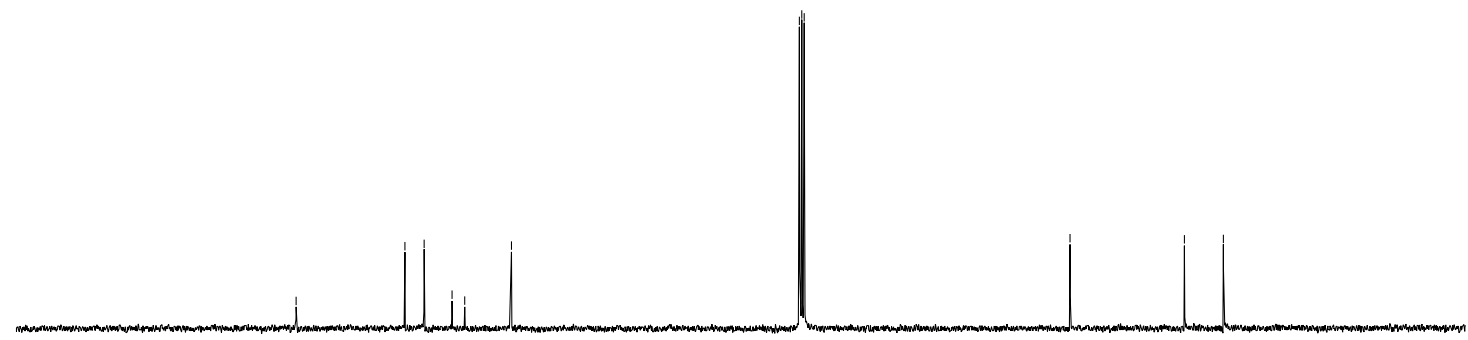

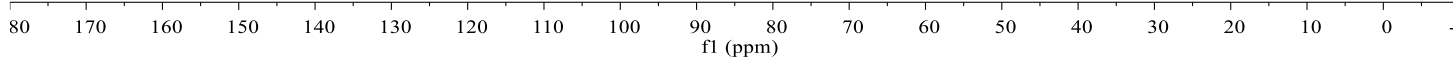


${ }^{1} \mathrm{H}$ NMR (400 MHz, $\left.\mathrm{CDCl}_{3}\right)$ spectrum of $\mathbf{4 j}$

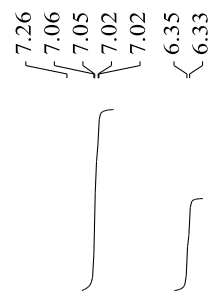

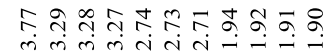

inm

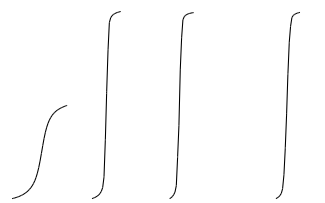<smiles>Brc1ccc2c(c1)CCCN2</smiles>

$4 \mathbf{j}$

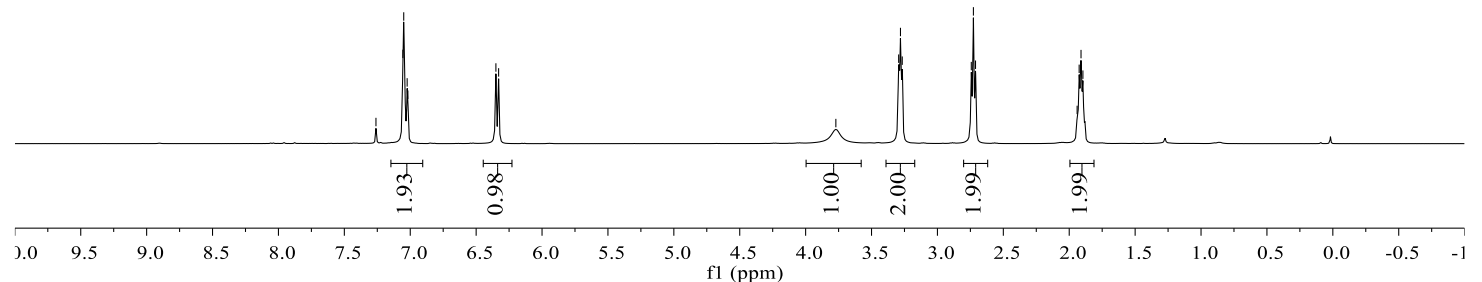

${ }^{13} \mathrm{C}\left\{{ }^{1} \mathrm{H}\right\}$ NMR $\left(101 \mathrm{MHz}, \mathrm{CDCl}_{3}\right)$ spectrum of $\mathbf{4} \mathbf{j}$

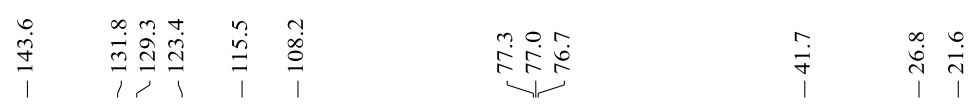<smiles>Brc1ccc2c(c1)CCCN2</smiles>

$4 \mathbf{j}$

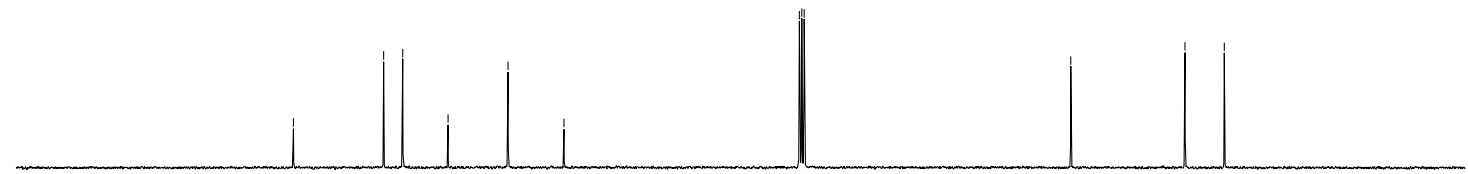

$\begin{array}{lllllllllllllllllll}80 & 170 & 160 & 150 & 140 & 130 & 120 & 110 & 100 & \begin{array}{c}90 \\ \mathrm{f} 1(\mathrm{ppm})\end{array} & 70 & 60 & 50 & 40 & 30 & 20 & 10 & 0 & \end{array}$ 
${ }^{1} \mathrm{H}$ NMR $\left(400 \mathrm{MHz}, \mathrm{CDCl}_{3}\right)$ spectrum of $\mathbf{4 k}$

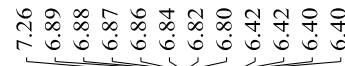

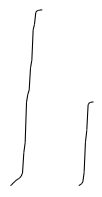

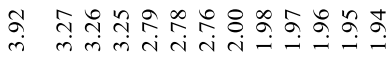

皮

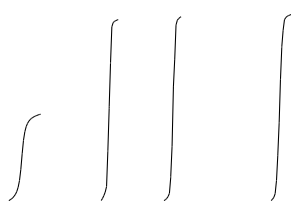<smiles>Brc1cccc2c1CCCN2</smiles>

$\mathbf{4 k}$

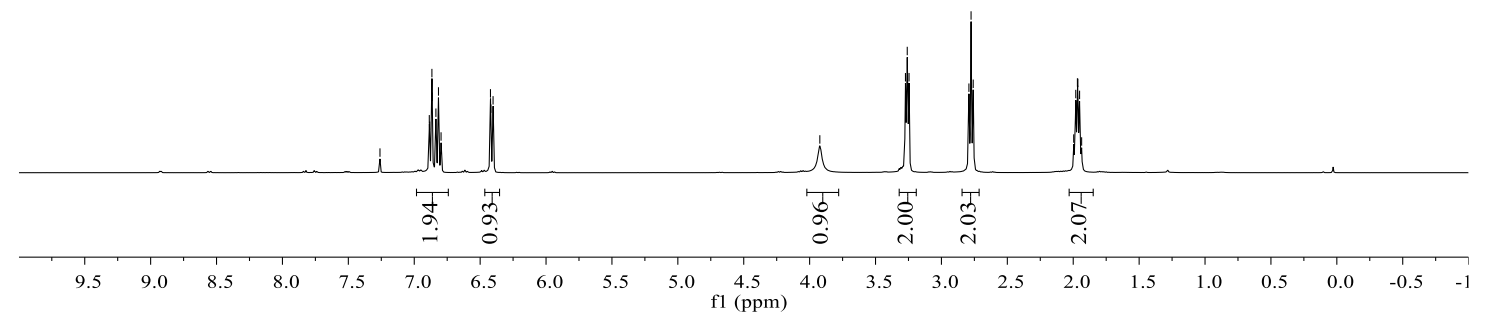

${ }^{13} \mathrm{C}\left\{{ }^{1} \mathrm{H}\right\}$ NMR $\left(101 \mathrm{MHz}, \mathrm{CDCl}_{3}\right)$ spectrum of $\mathbf{4 k}$

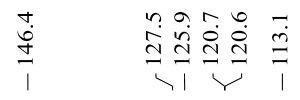

齐点宾

$\begin{array}{lll}1 & 1 & 1\end{array}$

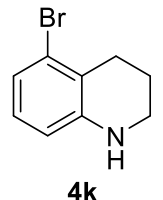

$4 \mathbf{k}$

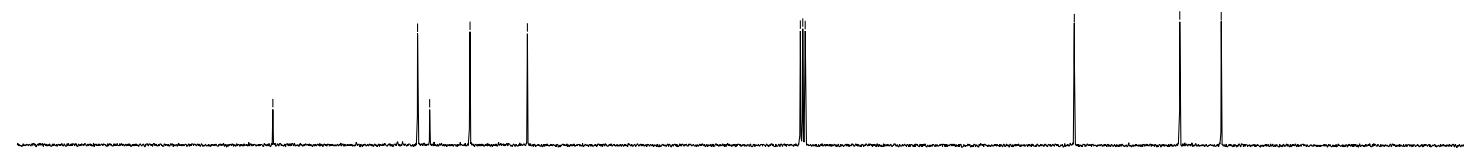

$\begin{array}{llllllllll}80 & 170 & 160 & 150 & 140 & 130 & 120 & 110 & 100 & 90 \quad 80 \\ \mathrm{f} 1(\mathrm{ppm})\end{array}$ 
${ }^{1} \mathrm{H}$ NMR $\left(400 \mathrm{MHz}, \mathrm{CDCl}_{3}\right)$ spectrum of $4 \mathbf{l}$
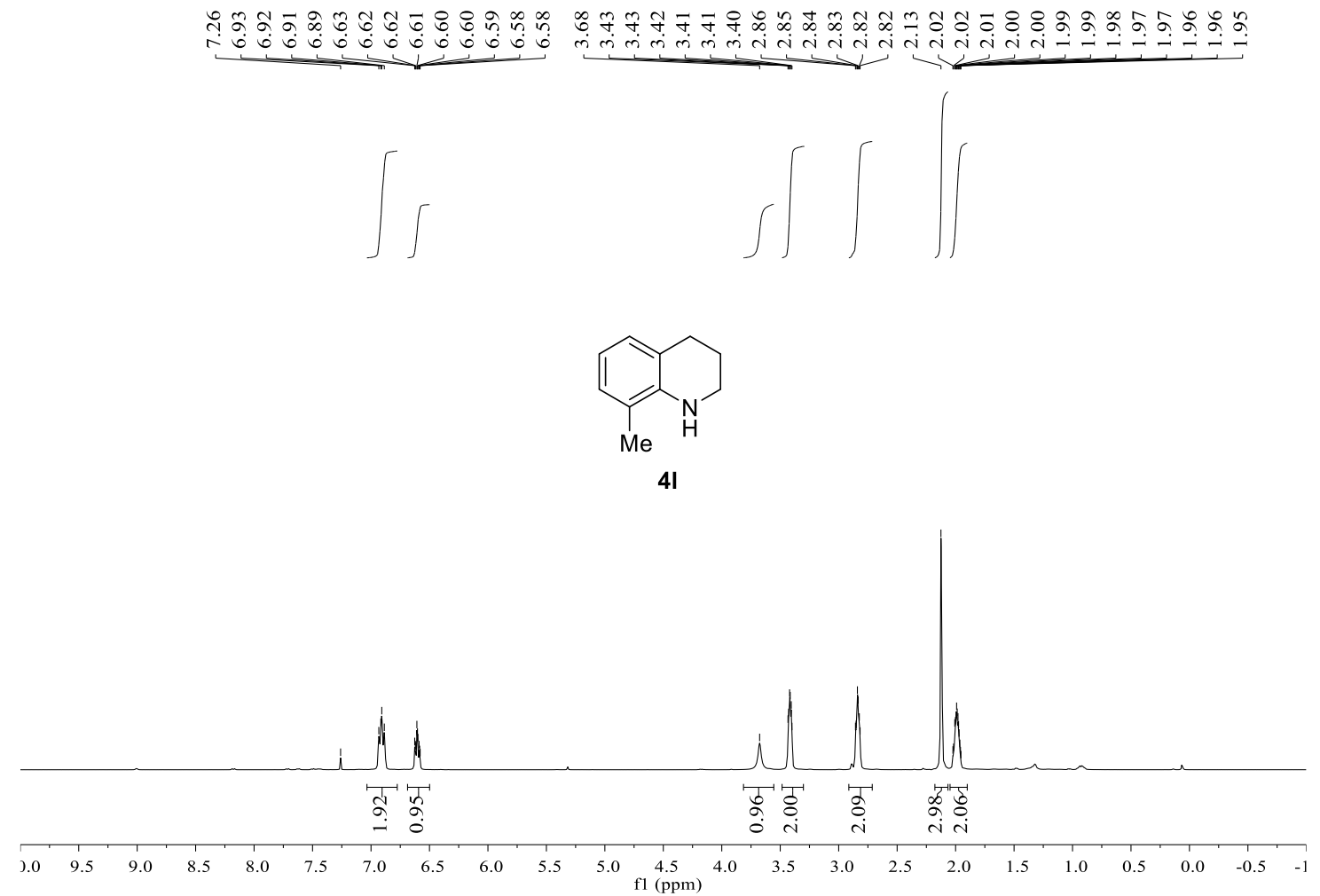

${ }^{13} \mathrm{C}\left\{{ }^{1} \mathrm{H}\right\}$ NMR $\left(101 \mathrm{MHz}, \mathrm{CDCl}_{3}\right)$ spectrum of $4 \mathrm{l}$

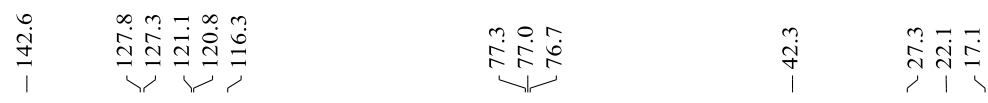
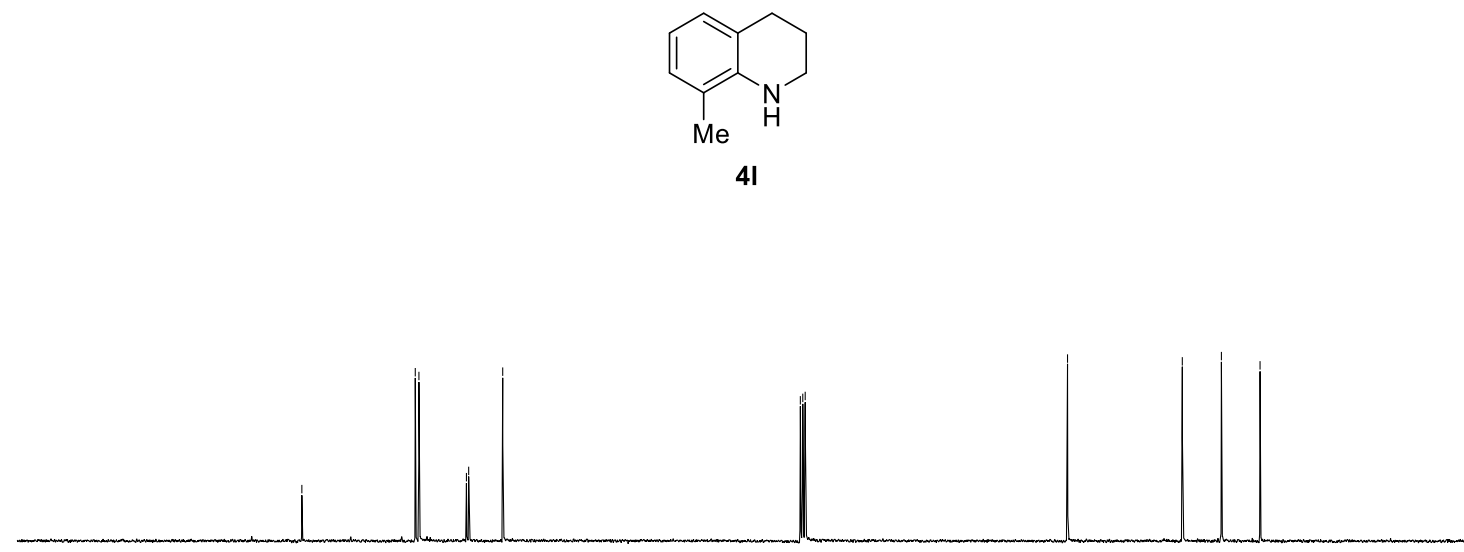

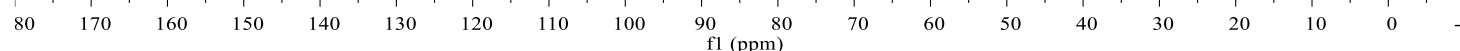


${ }^{1} \mathrm{H}$ NMR (400 MHz, $\mathrm{CDCl}_{3}$ ) spectrum of $\mathbf{4 m}$
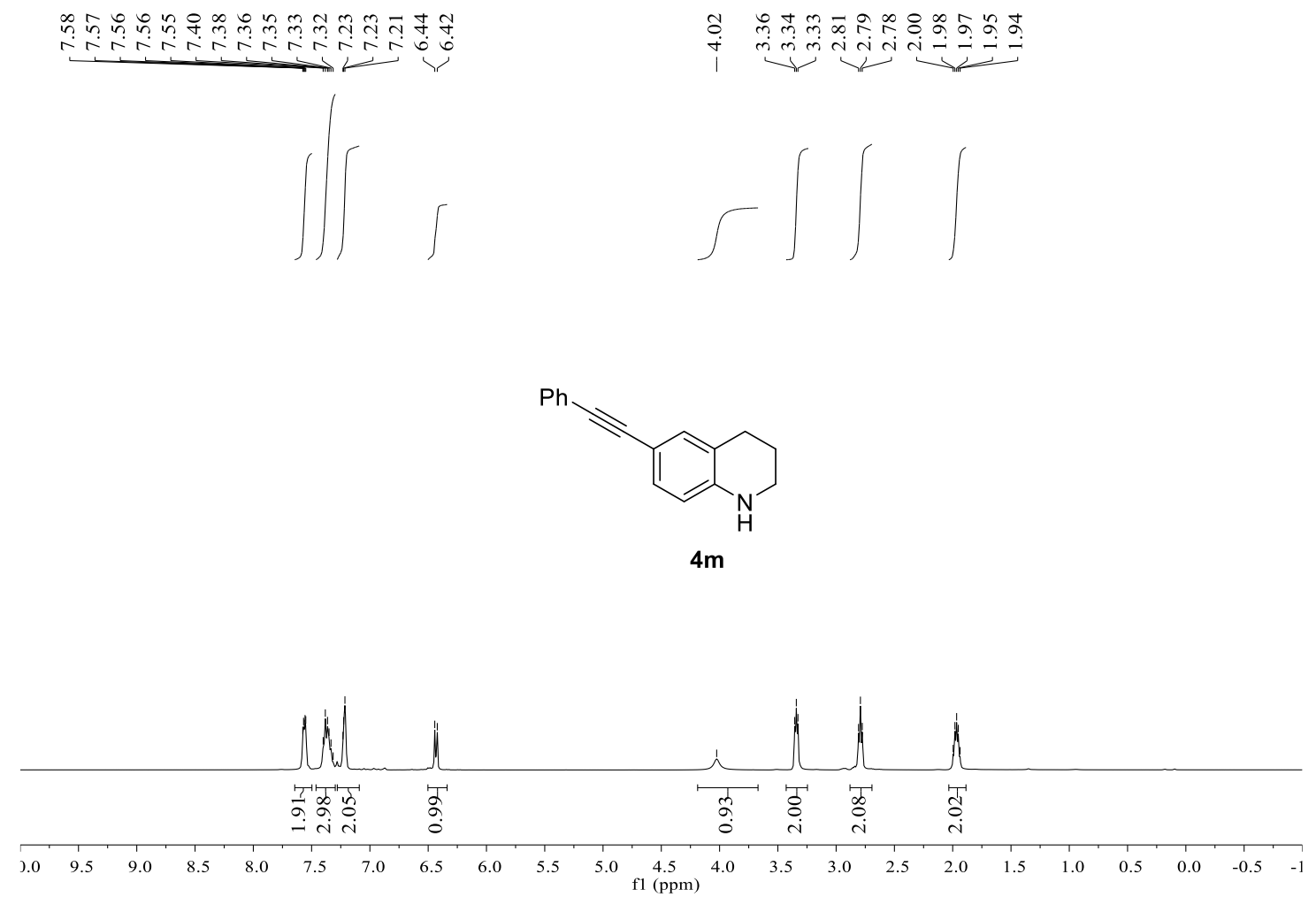

${ }^{13} \mathrm{C}\left\{{ }^{1} \mathrm{H}\right\}$ NMR $\left(101 \mathrm{MHz}, \mathrm{CDCl}_{3}\right)$ spectrum of $\mathbf{4 m}$

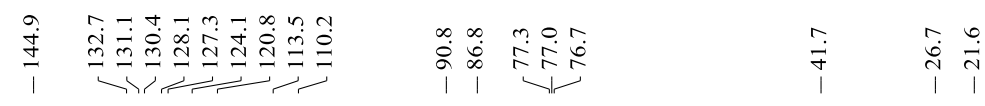
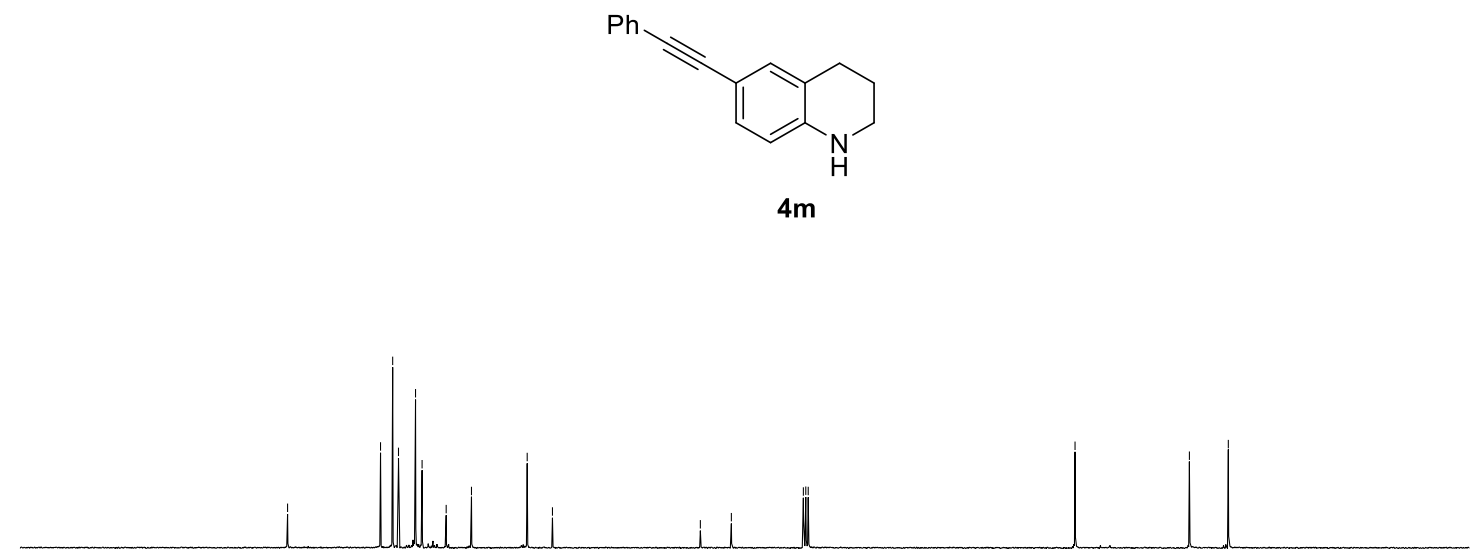

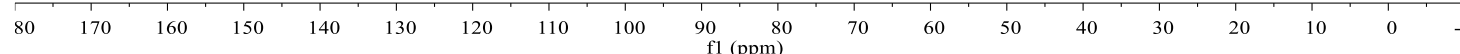


${ }^{1} \mathrm{H}$ NMR $\left(400 \mathrm{MHz}, \mathrm{CDCl}_{3}\right)$ spectrum of $\mathbf{4 n}$

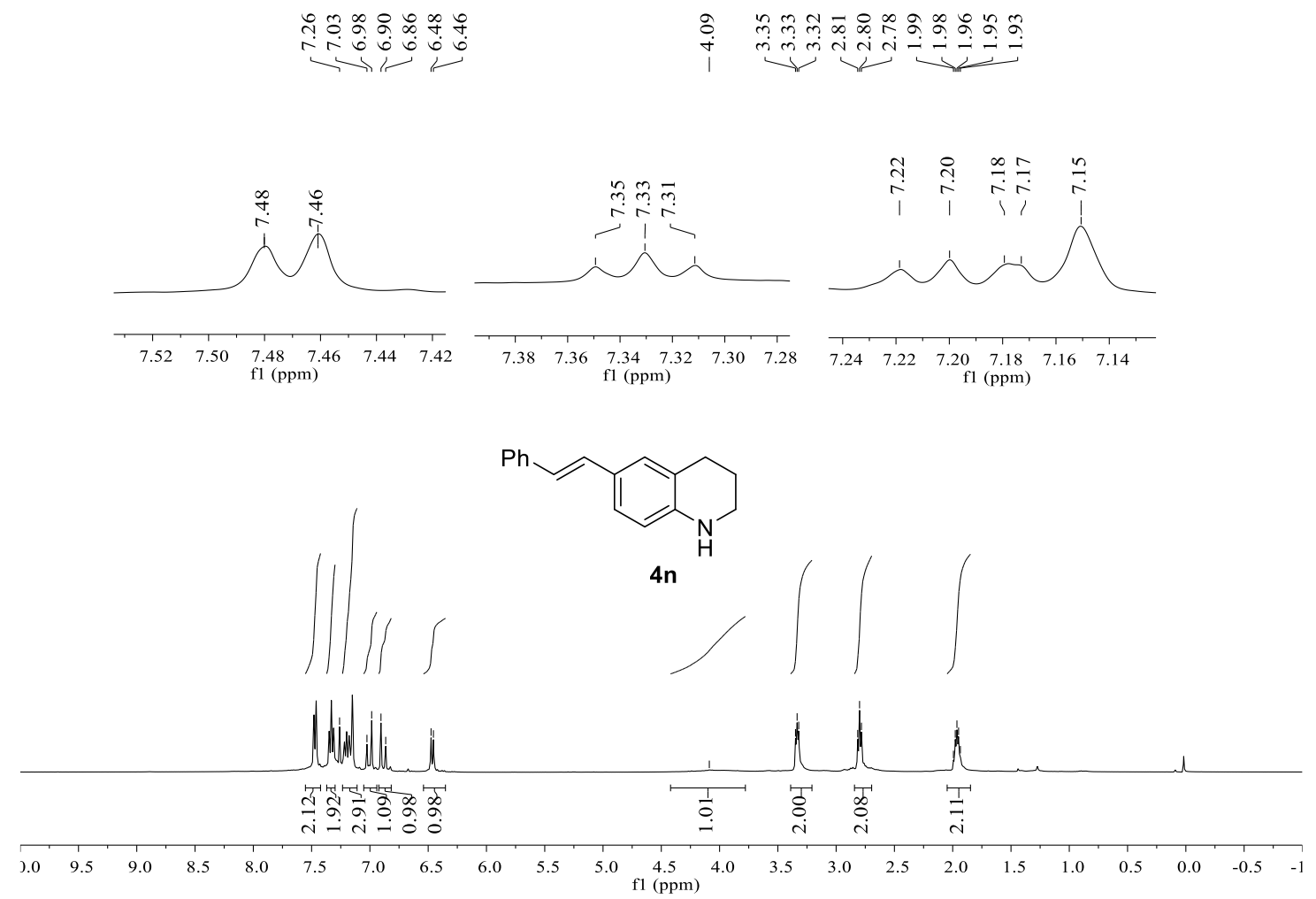

${ }^{13} \mathrm{C}\left\{{ }^{1} \mathrm{H}\right\}$ NMR $\left(101 \mathrm{MHz}, \mathrm{CDCl}_{3}\right)$ spectrum of $\mathbf{4 n}$

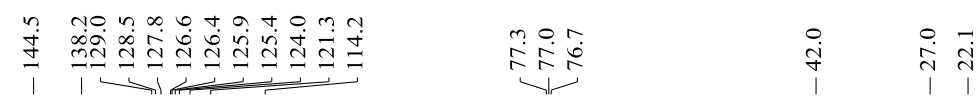<smiles>C(=C/c1ccc2c(c1)CCCN2)\c1ccccc1</smiles>

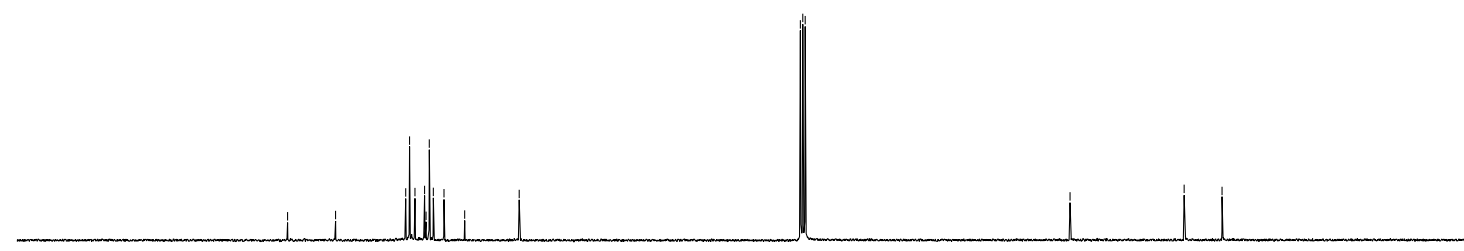

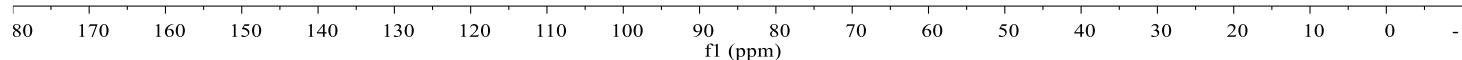


${ }^{1} \mathrm{H}$ NMR (400 MHz, $\mathrm{CDCl}_{3}$ ) spectrum of $4 \mathbf{o}$

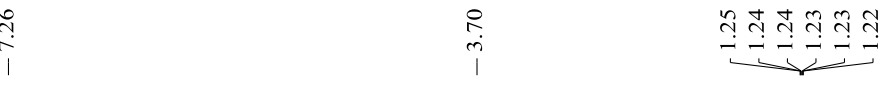

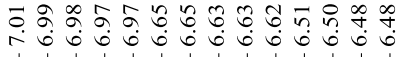

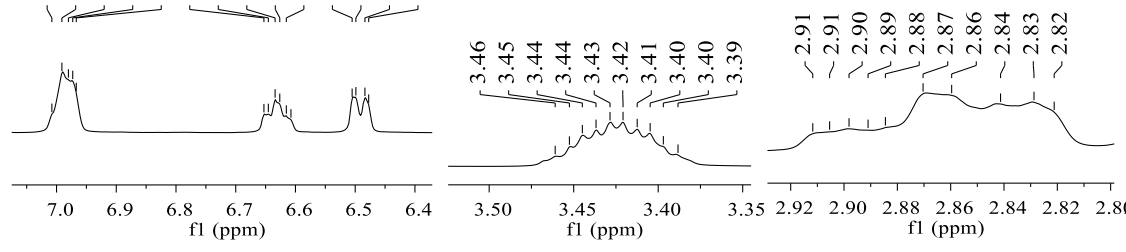

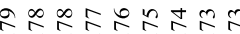
is

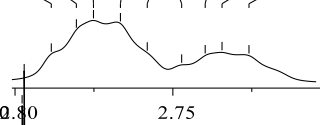

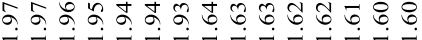

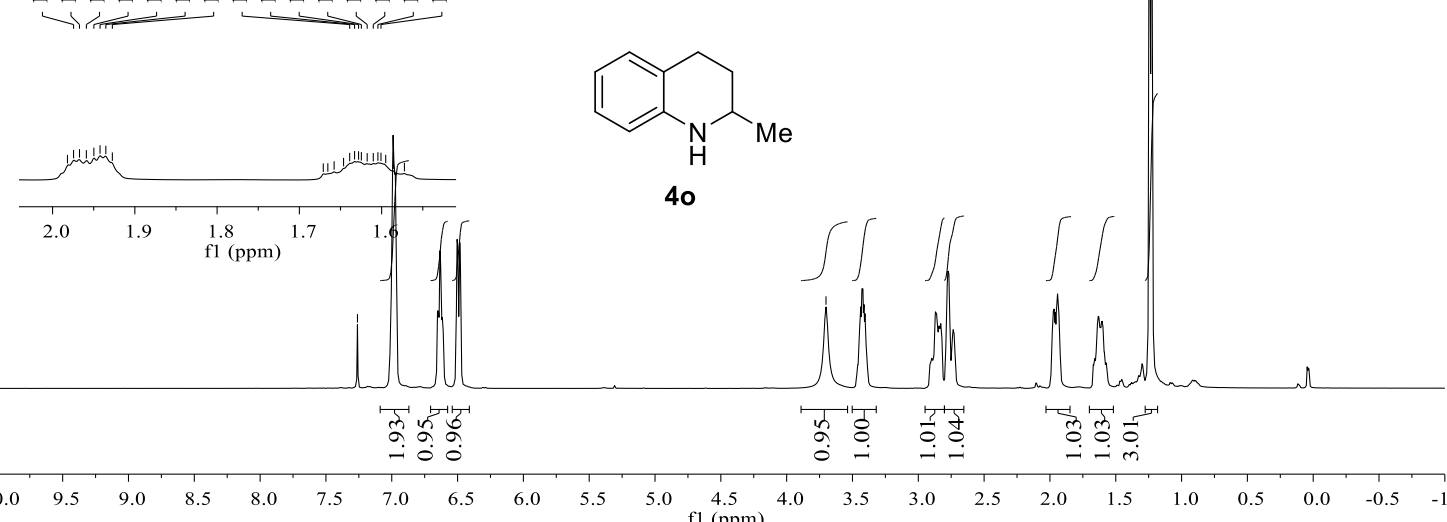

${ }^{13} \mathrm{C}\left\{{ }^{1} \mathrm{H}\right\}$ NMR $\left(101 \mathrm{MHz}, \mathrm{CDCl}_{3}\right)$ spectrum of $4 \mathbf{o}$

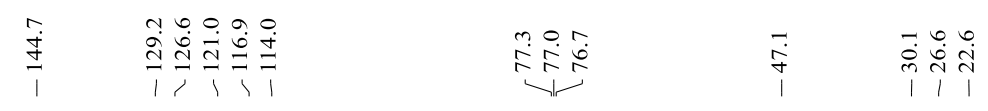
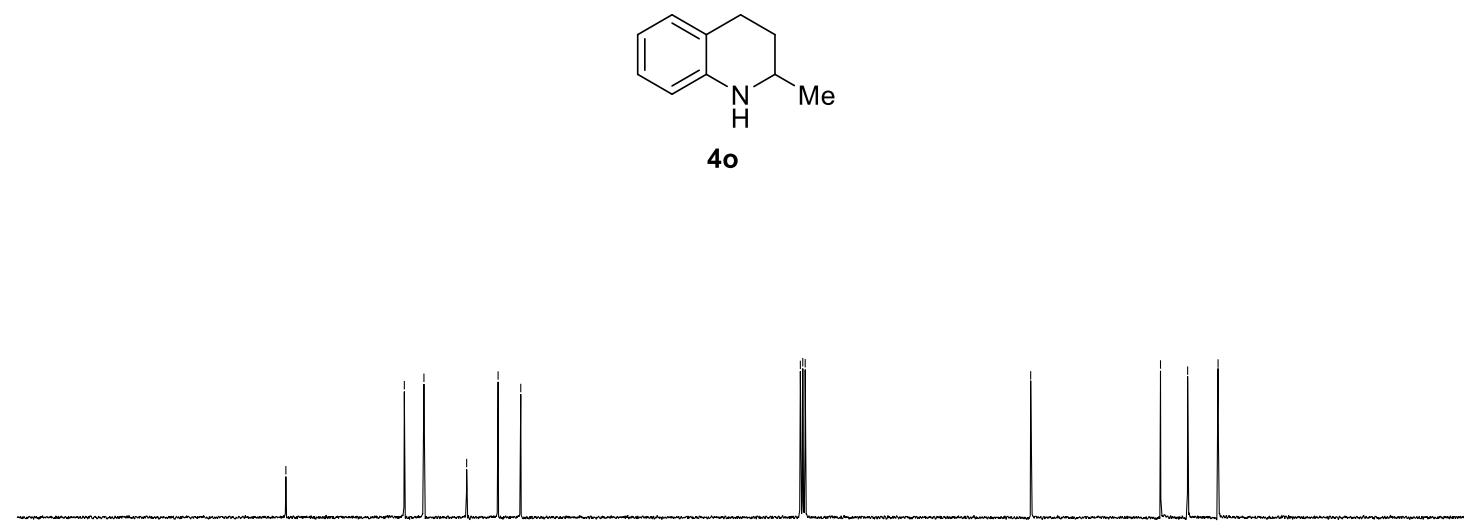

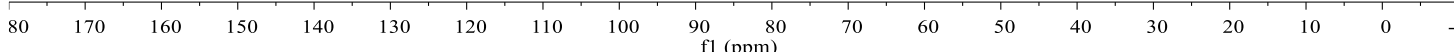


${ }^{1} \mathrm{H}$ NMR $\left(400 \mathrm{MHz}, \mathrm{CDCl}_{3}\right)$ spectrum of $\mathbf{4 p}$

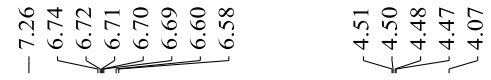

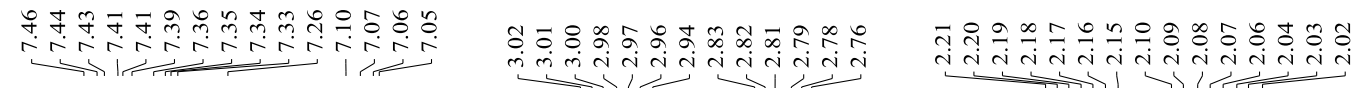
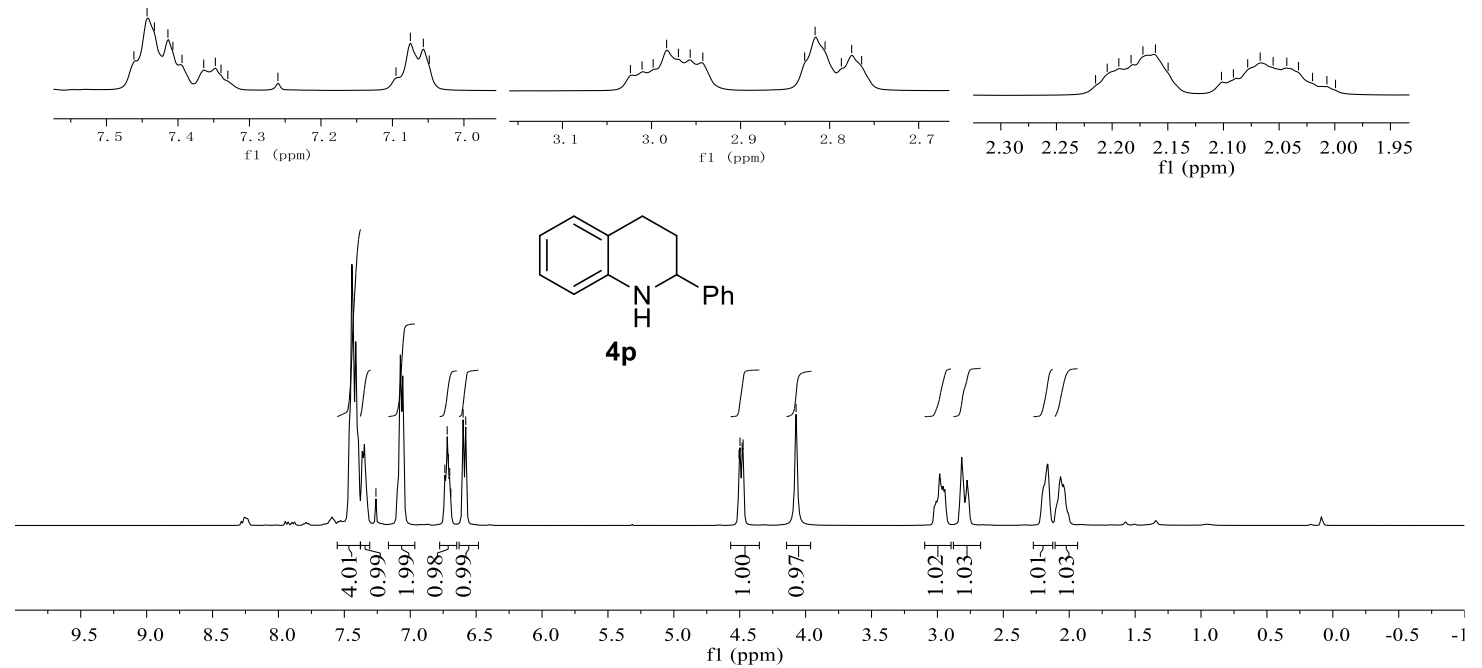

${ }^{13} \mathrm{C}\left\{{ }^{1} \mathrm{H}\right\}$ NMR $\left(101 \mathrm{MHz}, \mathrm{CDCl}_{3}\right)$ spectrum of $\mathbf{4 p}$

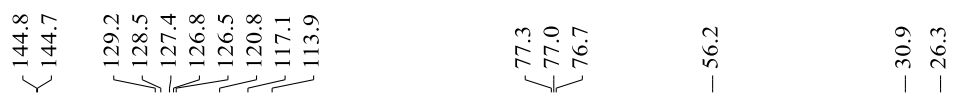
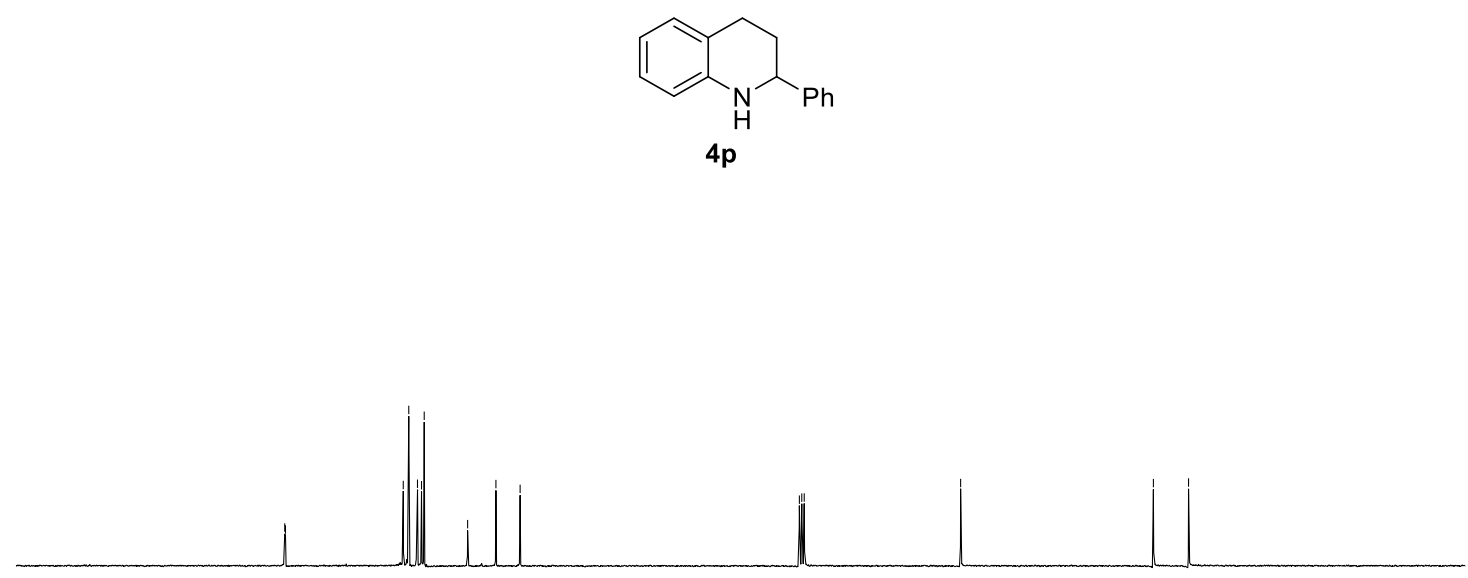

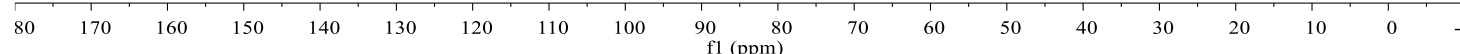


${ }^{1} \mathrm{H}$ NMR $\left(400 \mathrm{MHz}, \mathrm{CDCl}_{3}\right)$ spectrum of $\mathbf{4 q}$
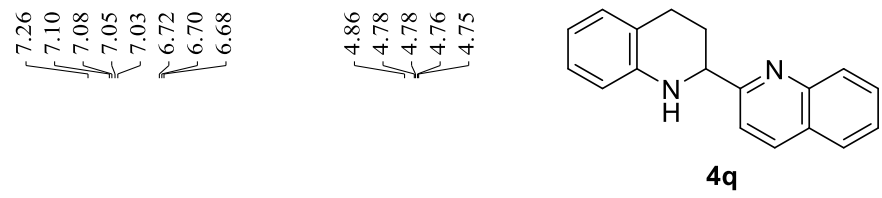

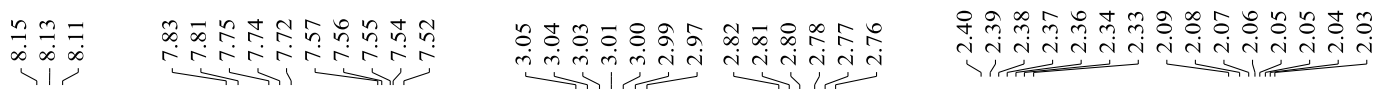
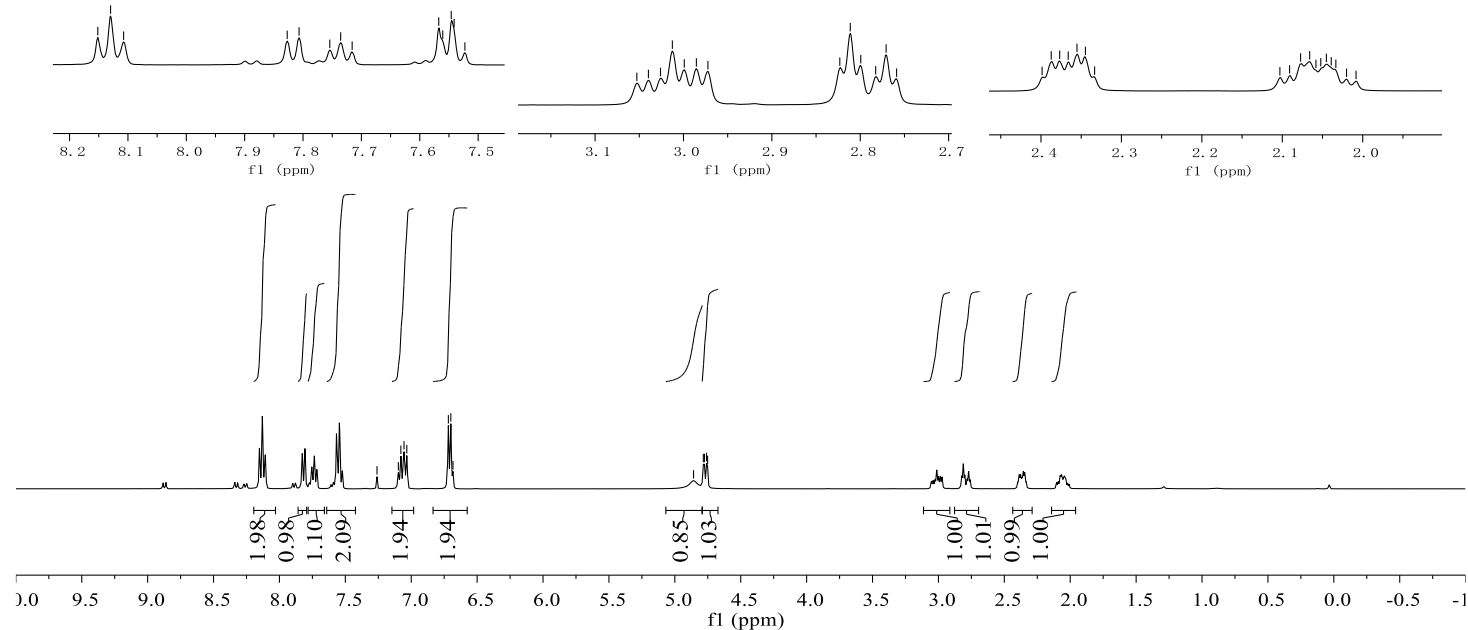

${ }^{13} \mathrm{C}\left\{{ }^{1} \mathrm{H}\right\}$ NMR $\left(101 \mathrm{MHz}, \mathrm{CDCl}_{3}\right)$ spectrum of $\mathbf{4 q}$

le

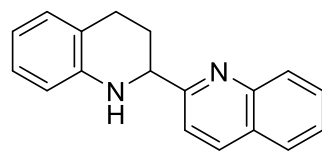

$4 q$

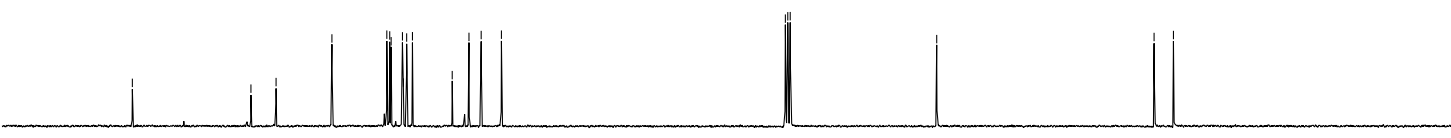

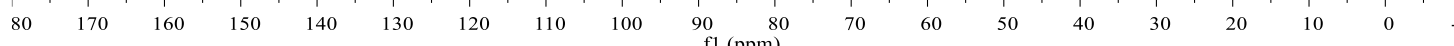


${ }^{1} \mathrm{H}$ NMR $\left(400 \mathrm{MHz}, \mathrm{CDCl}_{3}\right)$ spectrum of $\mathbf{4 r}$

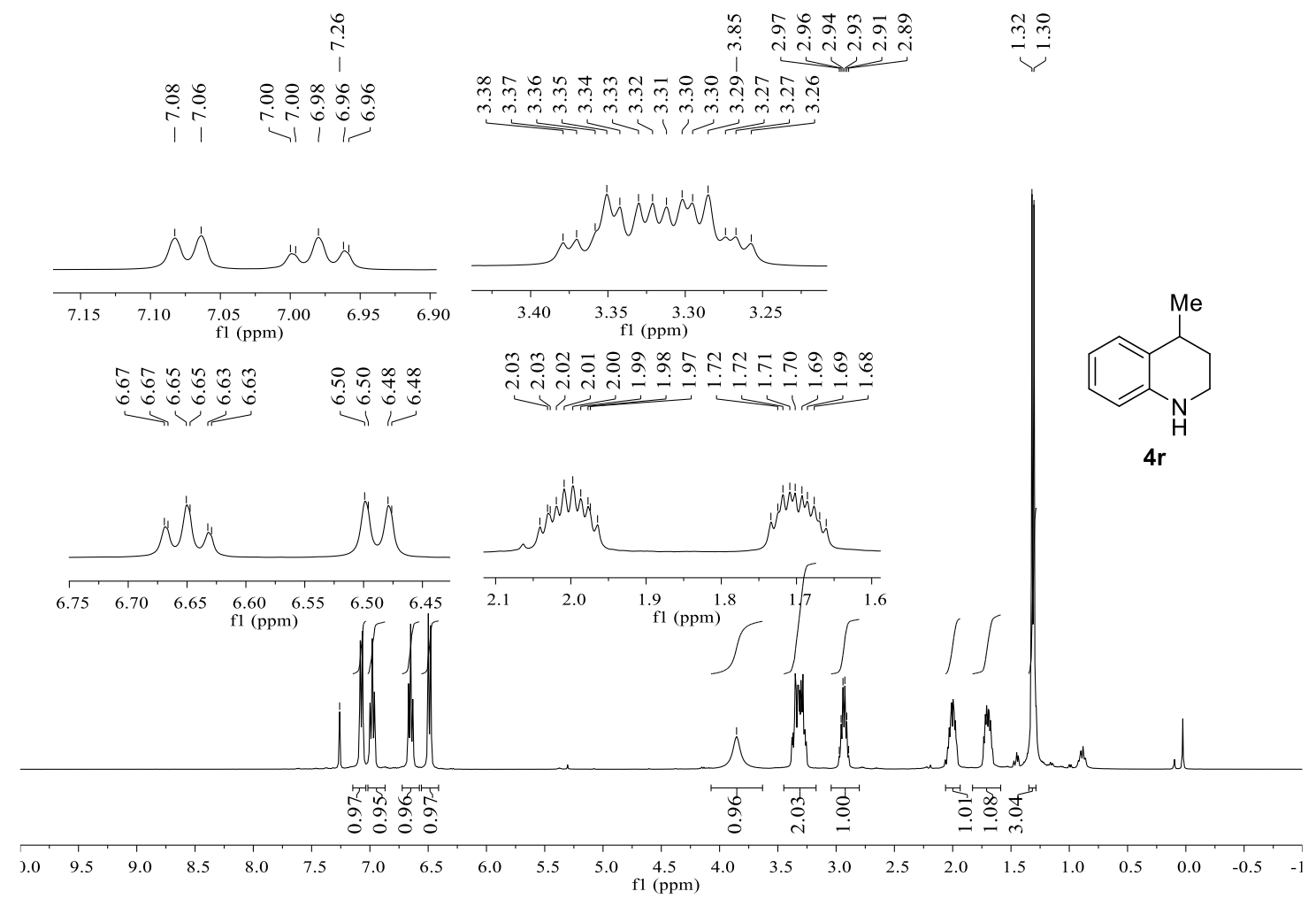

${ }^{13} \mathrm{C}\left\{{ }^{1} \mathrm{H}\right\}$ NMR $\left(101 \mathrm{MHz}, \mathrm{CDCl}_{3}\right)$ spectrum of $\mathbf{4 r}$
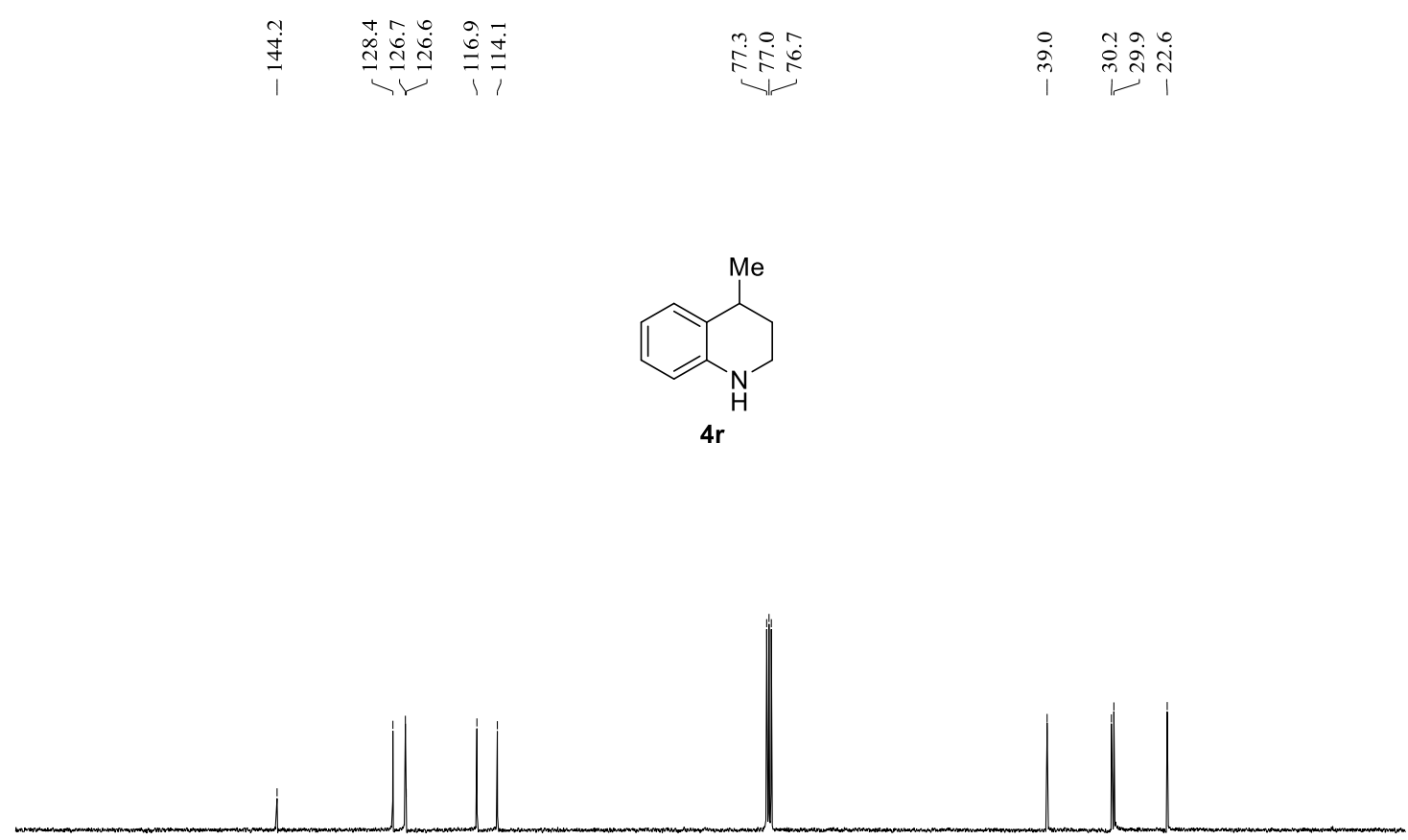

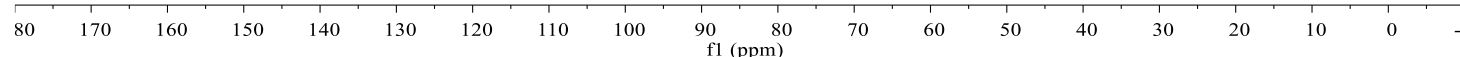


${ }^{1} \mathrm{H}$ NMR $\left(400 \mathrm{MHz}, \mathrm{CDCl}_{3}\right)$ spectrum of $\mathbf{4 s}$

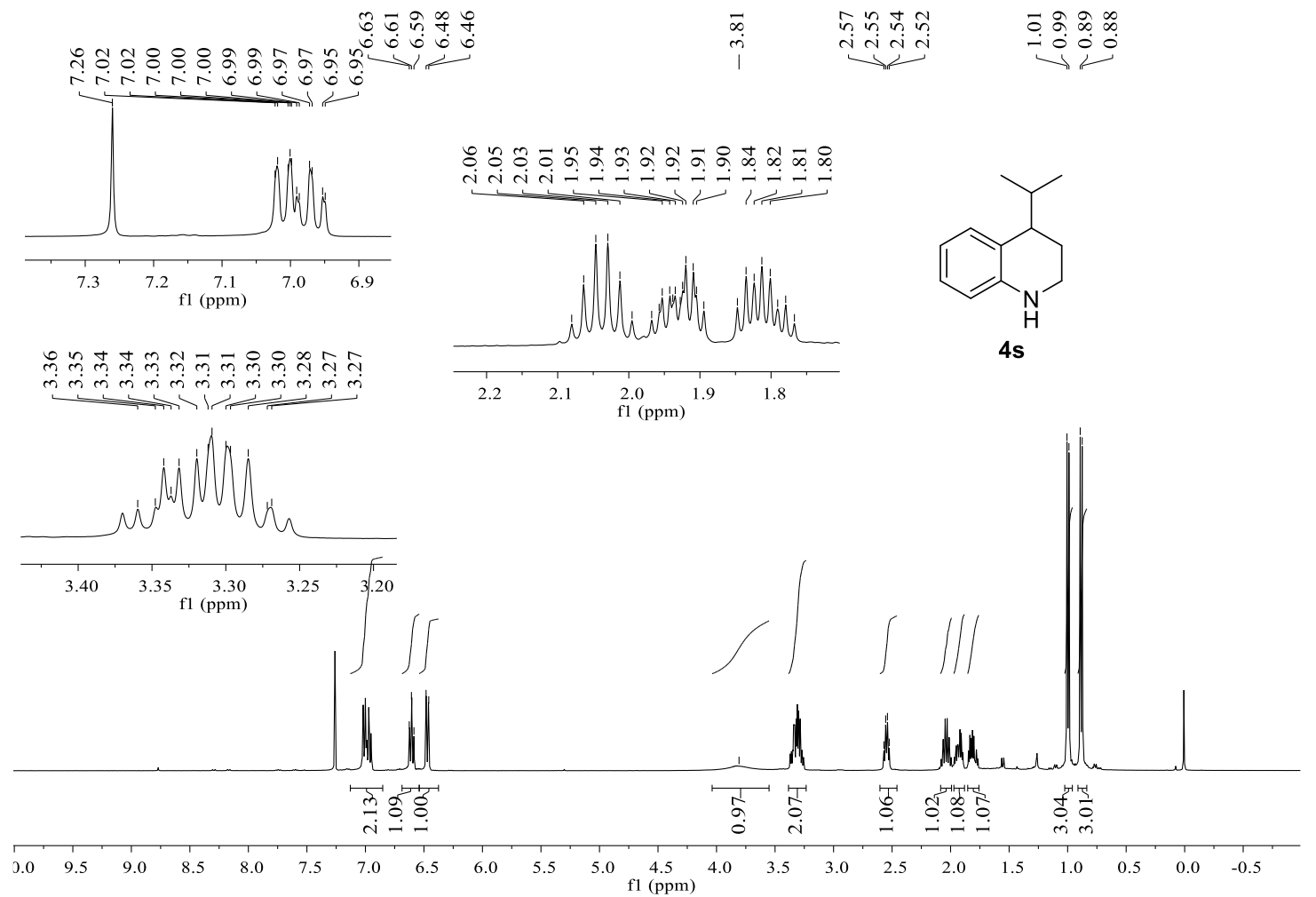

${ }^{13} \mathrm{C}\left\{{ }^{1} \mathrm{H}\right\}$ NMR $\left(101 \mathrm{MHz}, \mathrm{CDCl}_{3}\right)$ spectrum of $\mathbf{4 s}$
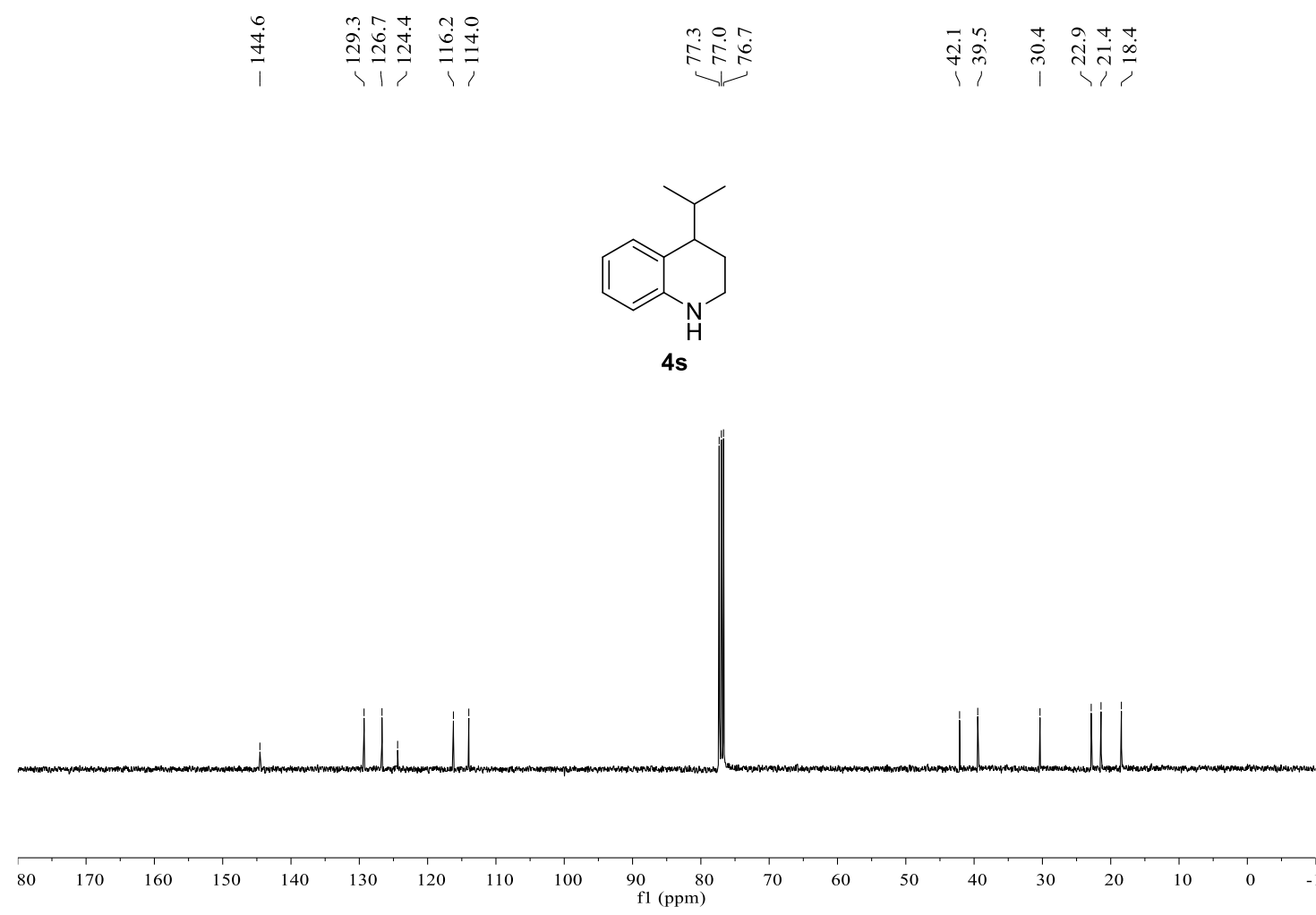
${ }^{1} \mathrm{H}$ NMR (400 MHz, $\mathrm{CDCl}_{3}$ ) spectrum of $\mathbf{4 t}$

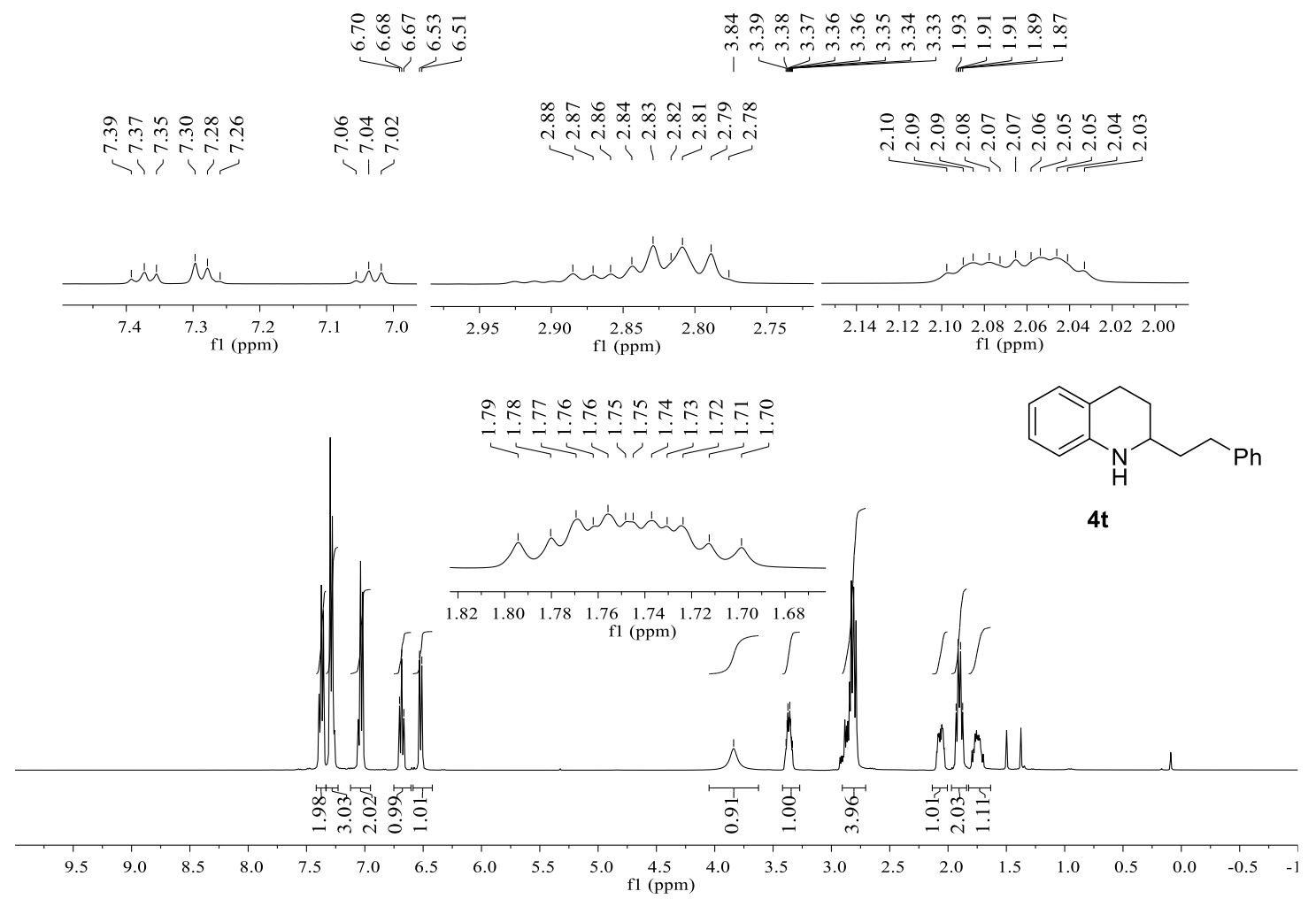

${ }^{13} \mathrm{C}\left\{{ }^{1} \mathrm{H}\right\}$ NMR (101 MHz, $\left.\mathrm{CDCl}_{3}\right)$ spectrum of $\mathbf{4 t}$

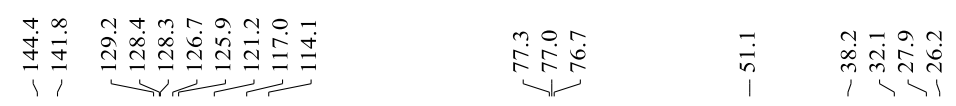

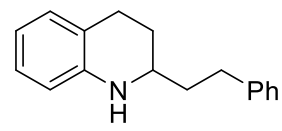

$4 t$

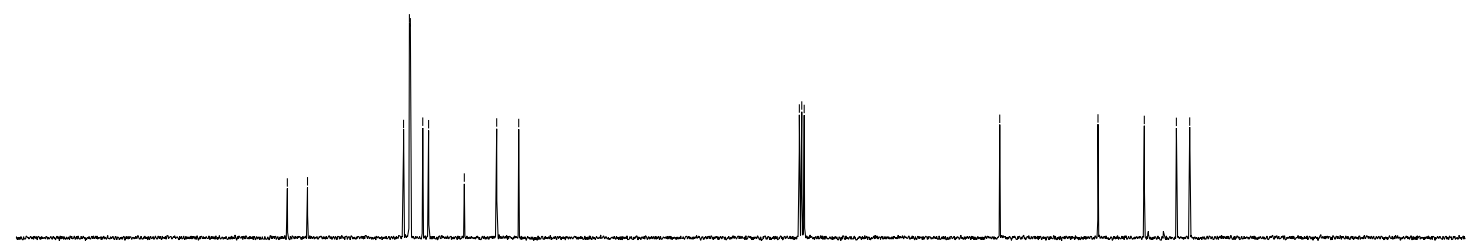

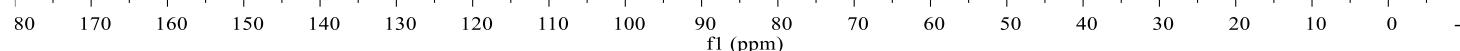


${ }^{1} \mathrm{H}$ NMR (400 MHz, $\left.\mathrm{CDCl}_{3}\right)$ spectrum of $\mathbf{4 u}$

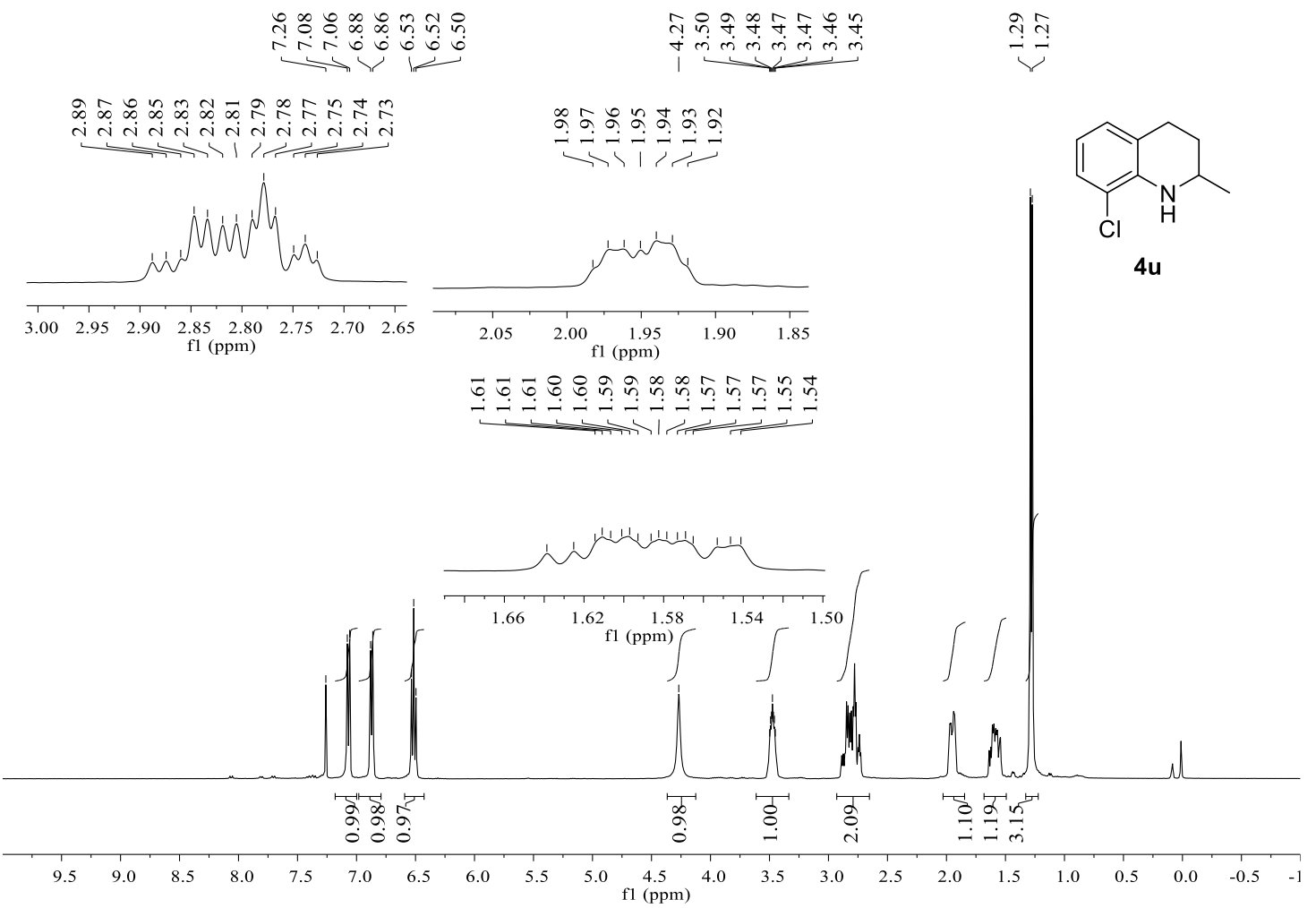

${ }^{13} \mathrm{C}\left\{{ }^{1} \mathrm{H}\right\}$ NMR $\left(101 \mathrm{MHz}, \mathrm{CDCl}_{3}\right)$ spectrum of $\mathbf{4 u}$

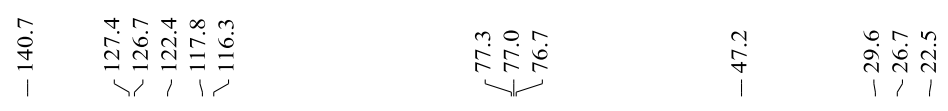

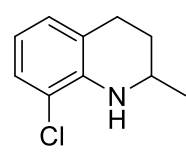

$4 \mathbf{u}$

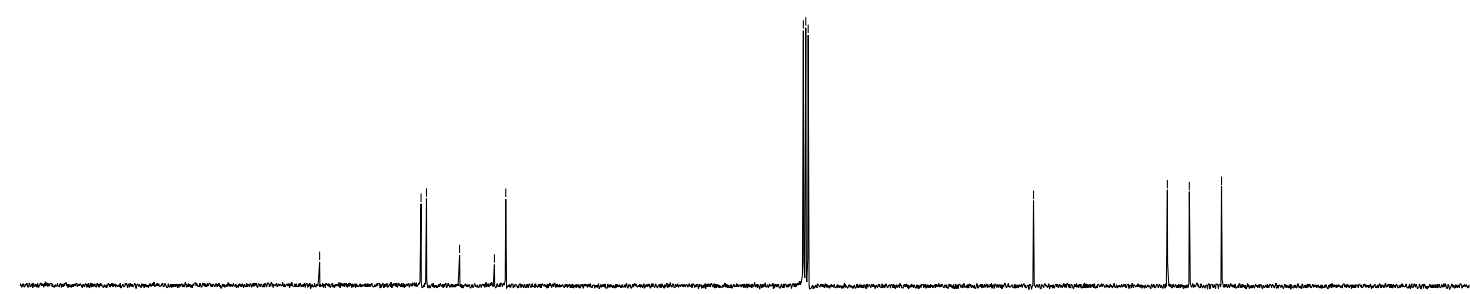

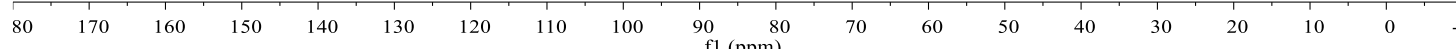


${ }^{1} \mathrm{H}$ NMR (400 MHz, $\mathrm{CDCl}_{3}$ ) spectrum of $\mathbf{6 a}$

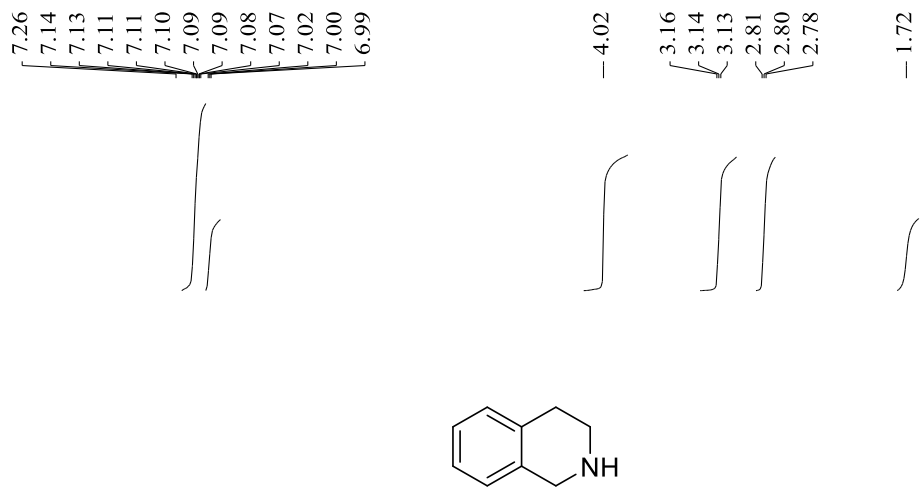

$6 \mathbf{a}$

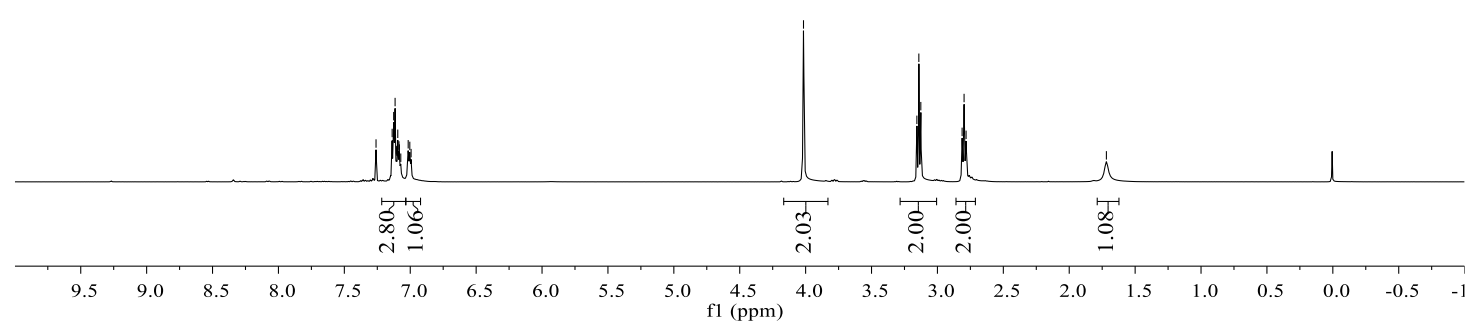

${ }^{13} \mathrm{C}\left\{{ }^{1} \mathrm{H}\right\}$ NMR $\left(101 \mathrm{MHz}, \mathrm{CDCl}_{3}\right)$ spectrum of $\mathbf{6 a}$

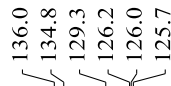

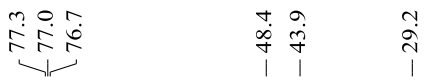

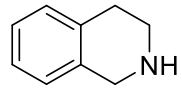

$6 a$

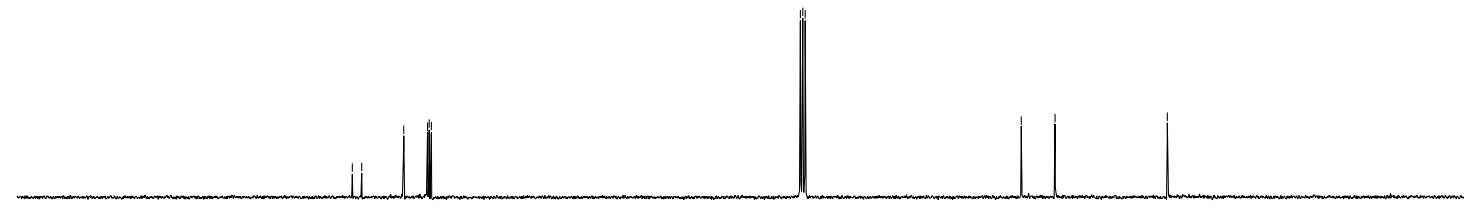

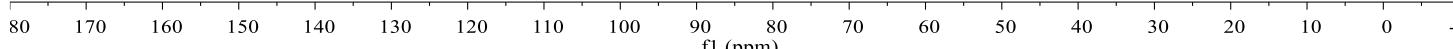


${ }^{1} \mathrm{H}$ NMR $\left(400 \mathrm{MHz}, \mathrm{CDCl}_{3}\right)$ spectrum of $\mathbf{6 b}$

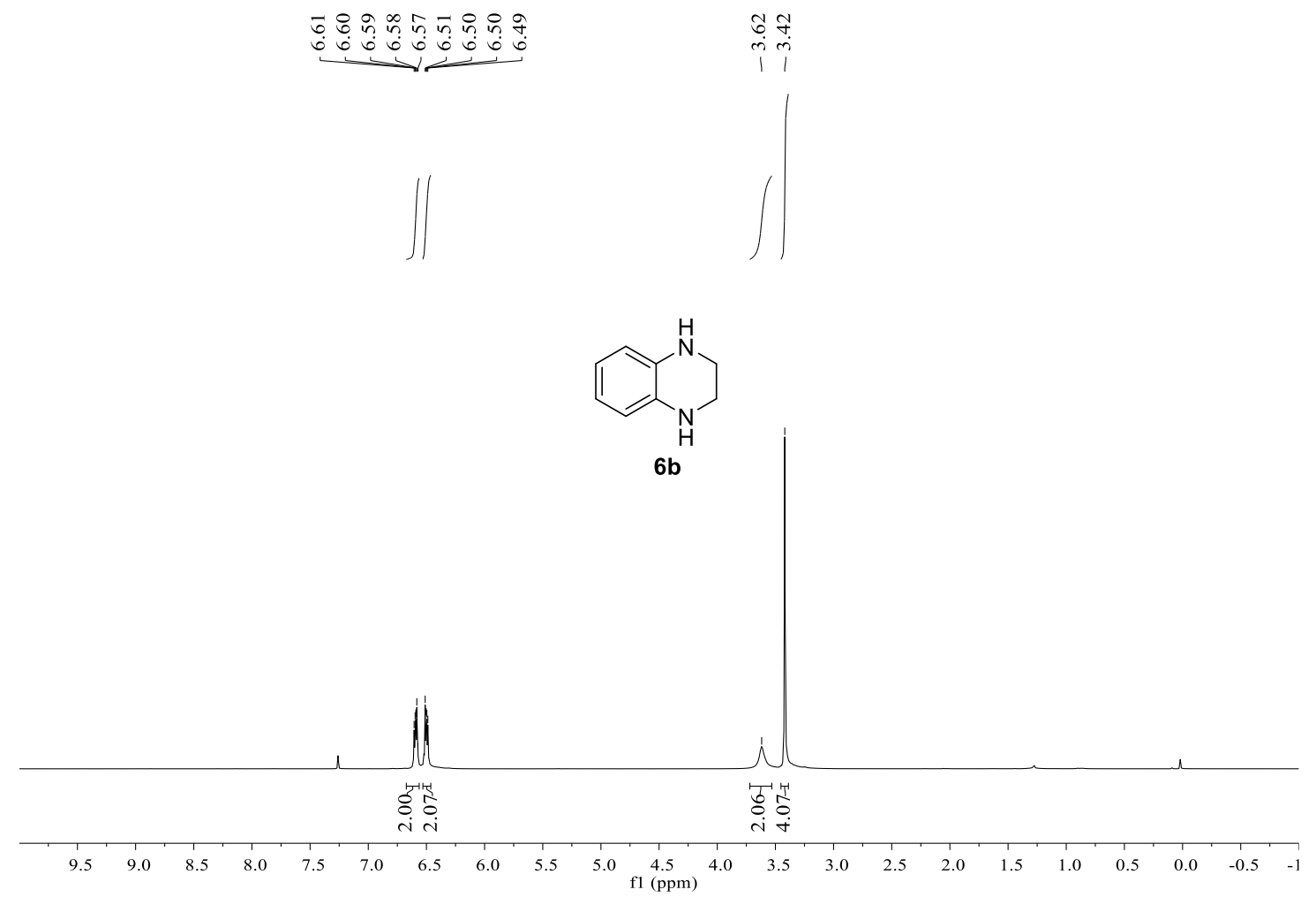

${ }^{13} \mathrm{C}\left\{{ }^{1} \mathrm{H}\right\}$ NMR $\left(101 \mathrm{MHz}, \mathrm{CDCl}_{3}\right)$ spectrum of $\mathbf{6 b}$
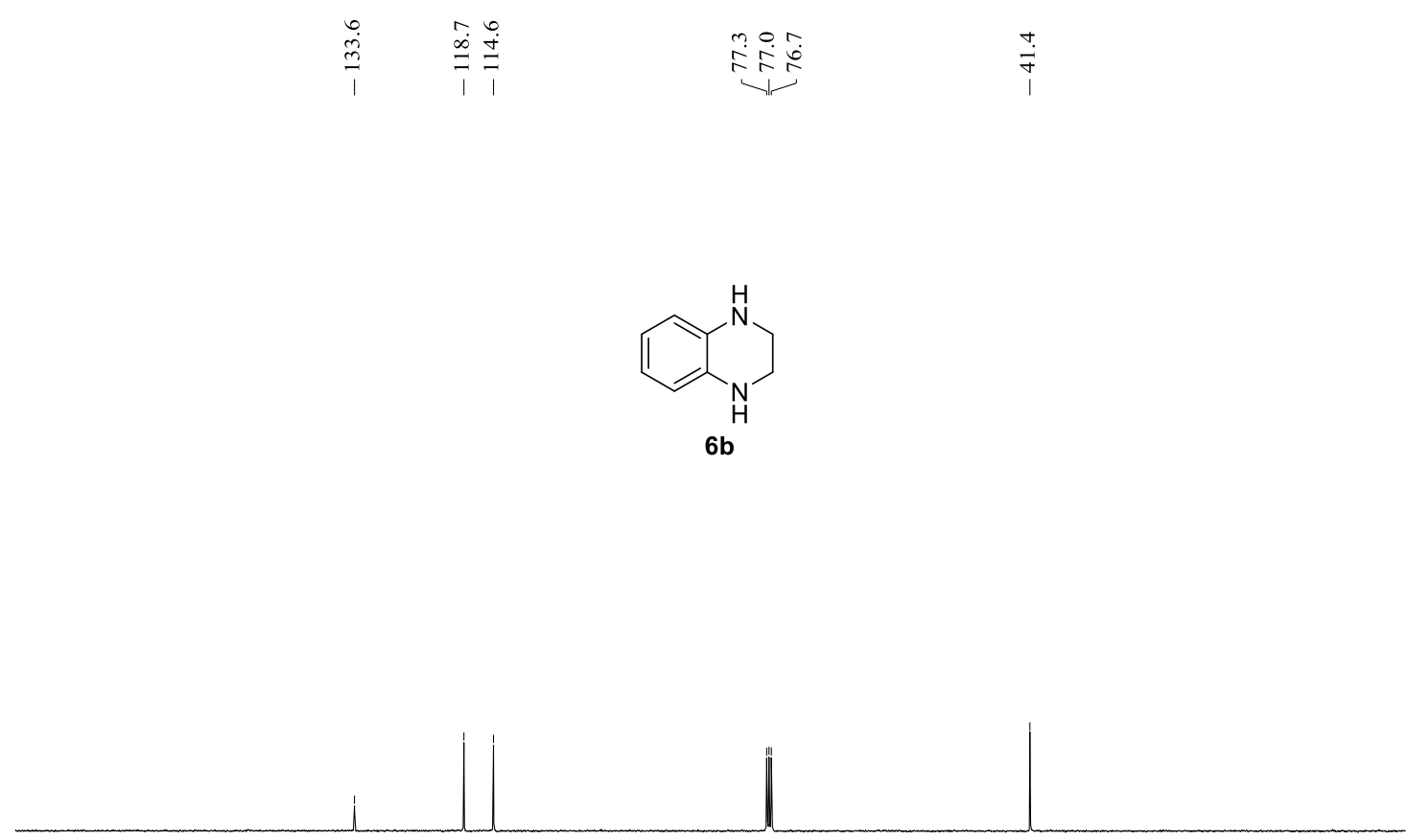

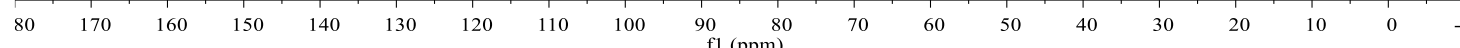


${ }^{1} \mathrm{H}$ NMR $\left(400 \mathrm{MHz}, \mathrm{CDCl}_{3}\right)$ spectrum of $\mathbf{6 c}$
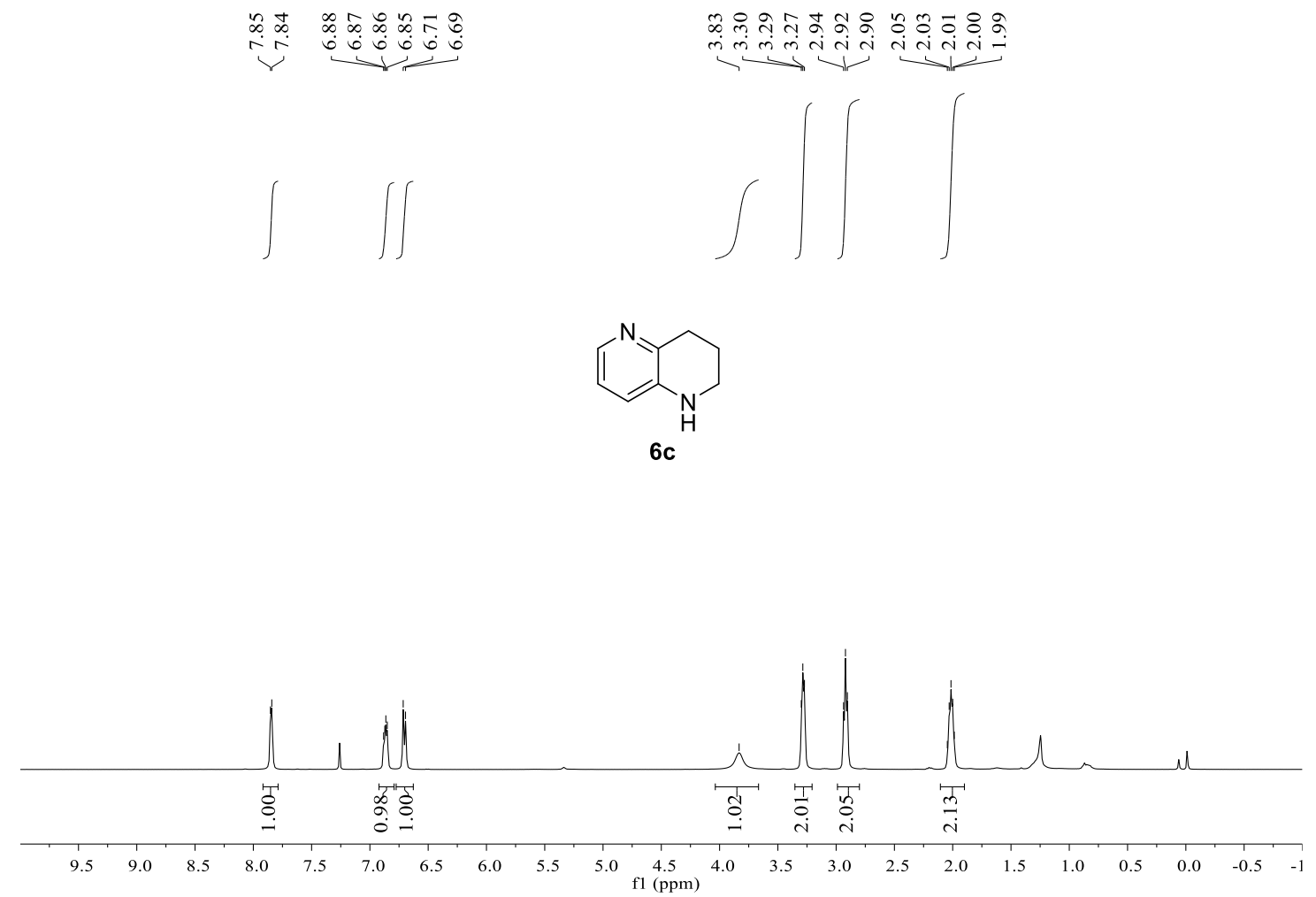

${ }^{13} \mathrm{C}\left\{{ }^{1} \mathrm{H}\right\}$ NMR $\left(101 \mathrm{MHz}, \mathrm{CDCl}_{3}\right)$ spectrum of $\mathbf{6 c}$
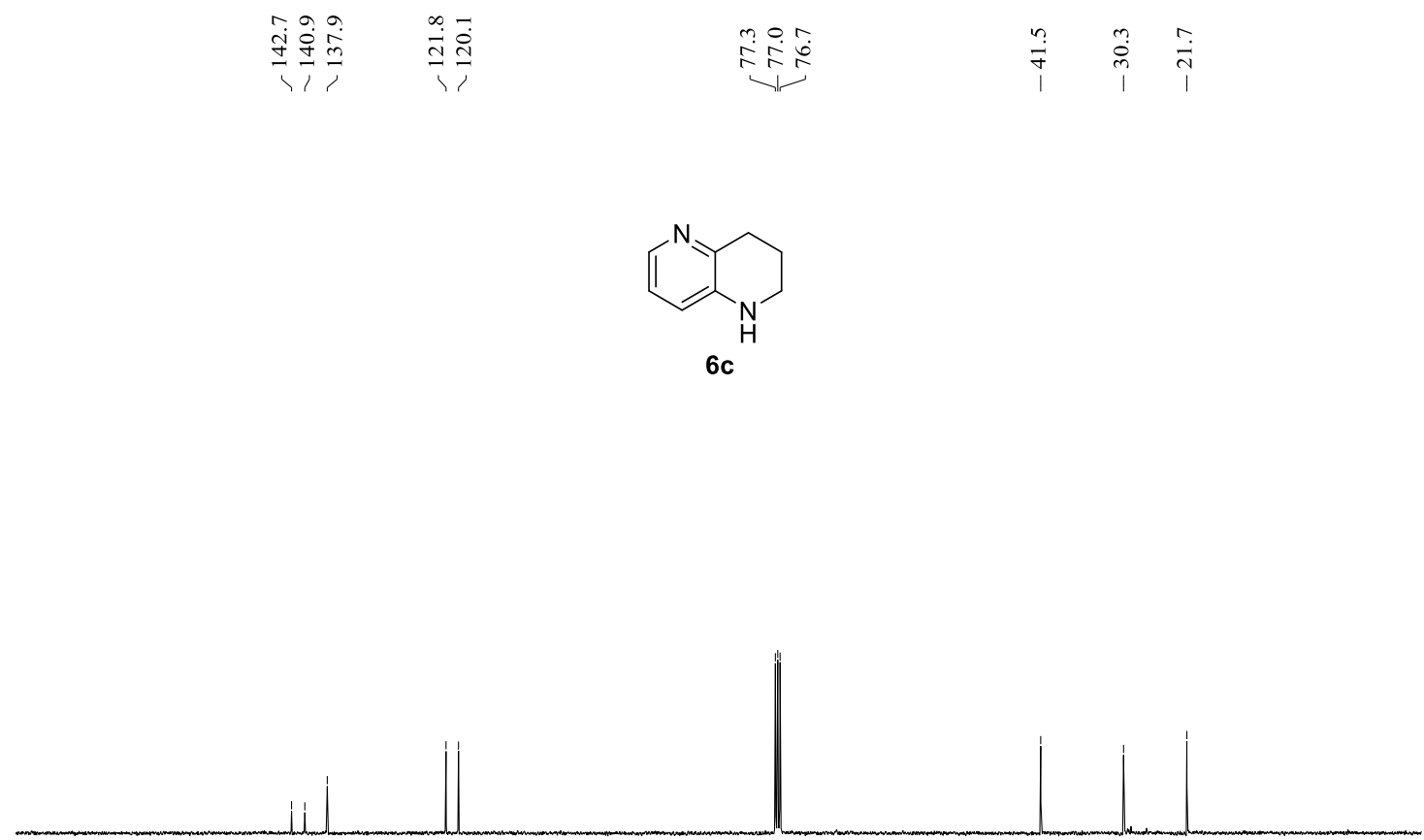

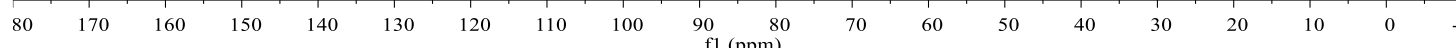


${ }^{1} \mathrm{H}$ NMR $\left(400 \mathrm{MHz}, \mathrm{CDCl}_{3}\right)$ spectrum of $\mathbf{6 d}$
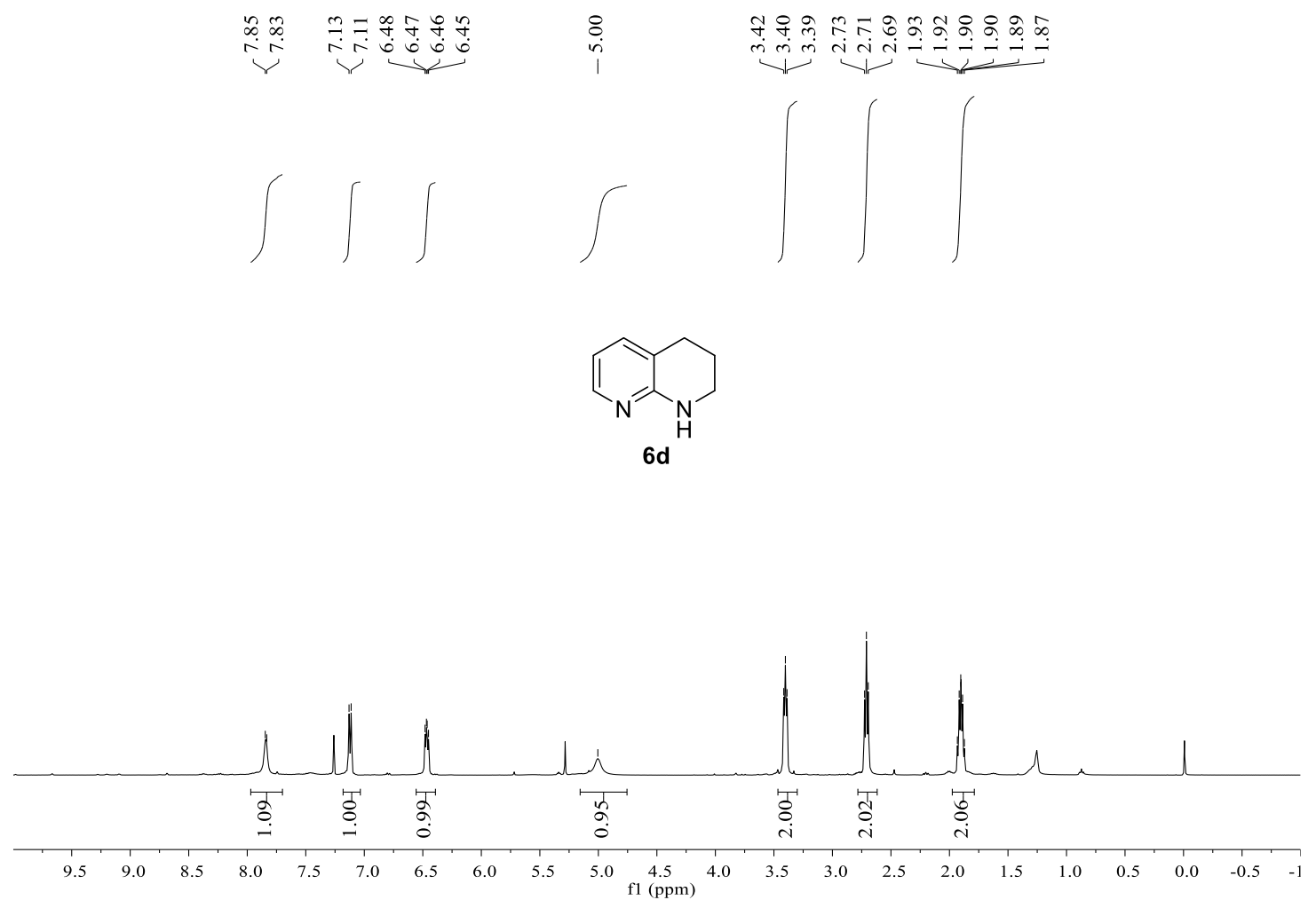

${ }^{13} \mathrm{C}\left\{{ }^{1} \mathrm{H}\right\}$ NMR $\left(101 \mathrm{MHz}, \mathrm{CDCl}_{3}\right)$ spectrum of $\mathbf{6 d}$

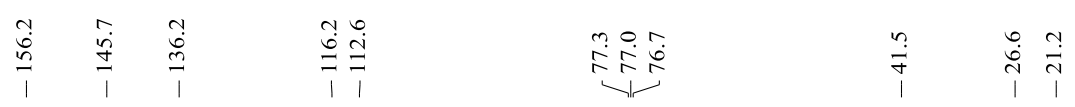
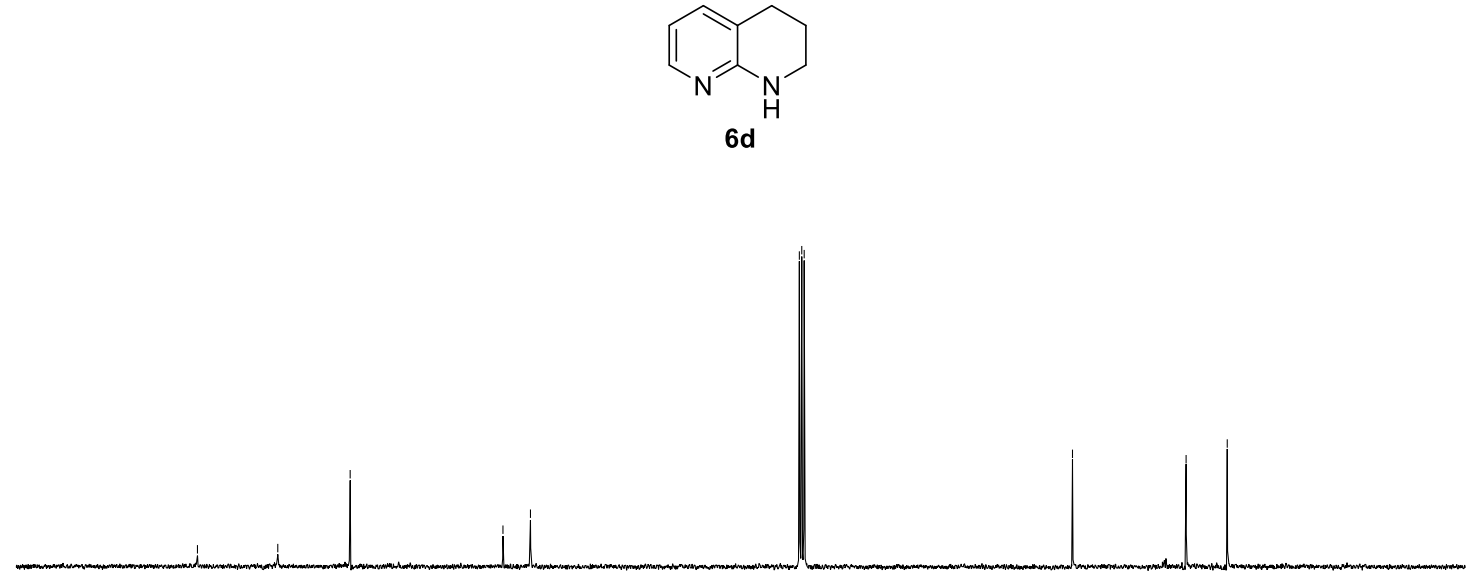

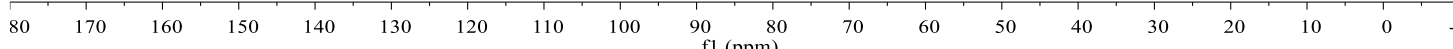


${ }^{1} \mathrm{H}$ NMR $\left(400 \mathrm{MHz}, \mathrm{CDCl}_{3}\right.$ ) spectrum of $\mathbf{6 e}$

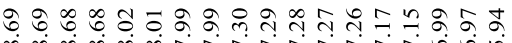

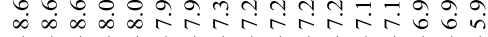

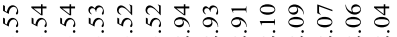

$\operatorname{minmminisinsiris~}$

$\iiint \iint$<smiles>c1cnc2c3c(ccc2c1)CCCN3</smiles>
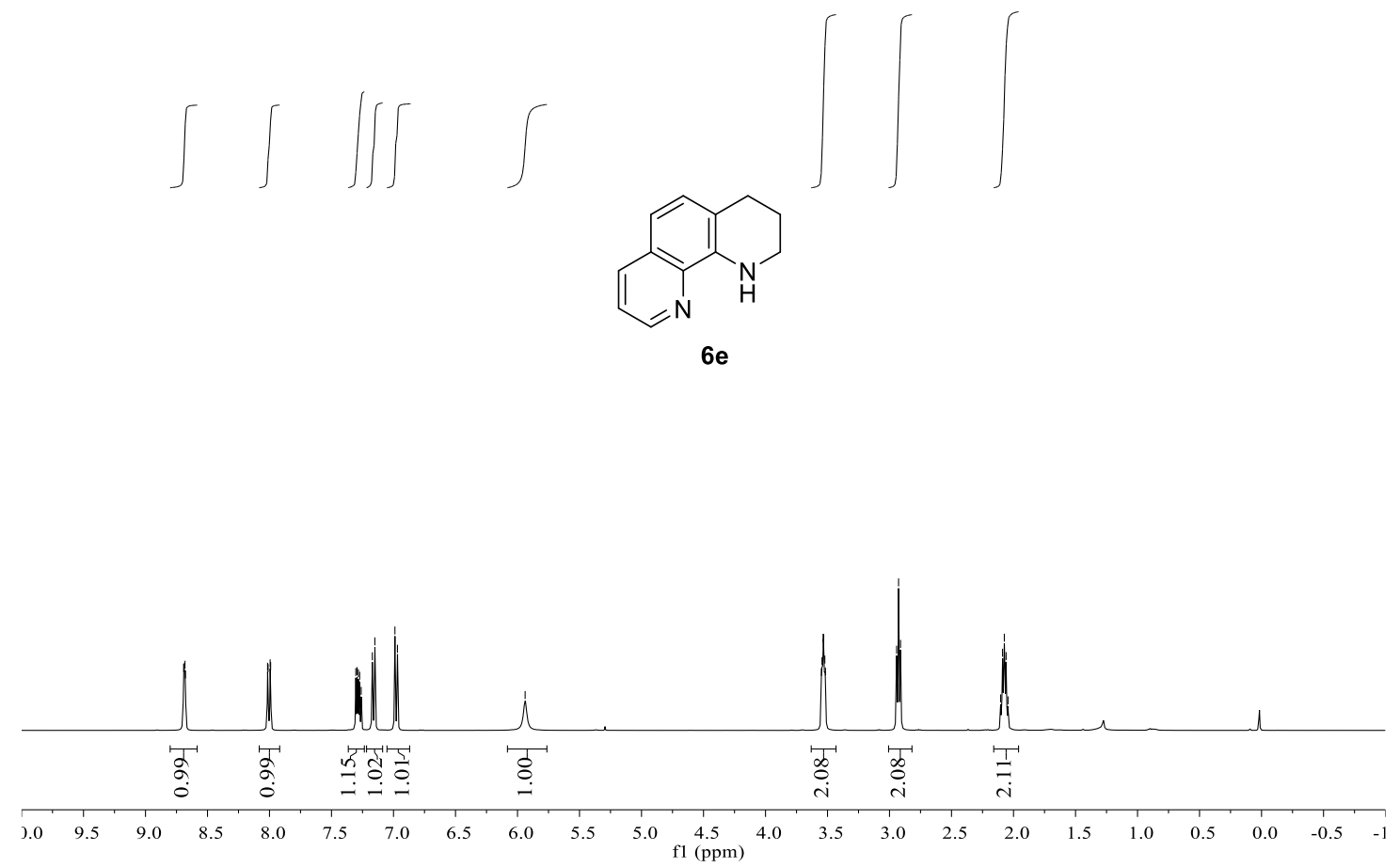

${ }^{13} \mathrm{C}\left\{{ }^{1} \mathrm{H}\right\}$ NMR $\left(101 \mathrm{MHz}, \mathrm{CDCl}_{3}\right)$ spectrum of $\mathbf{6 e}$

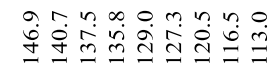

-

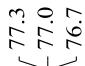

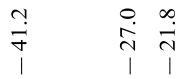

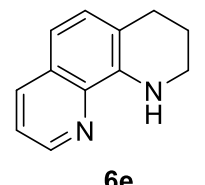

$6 e$

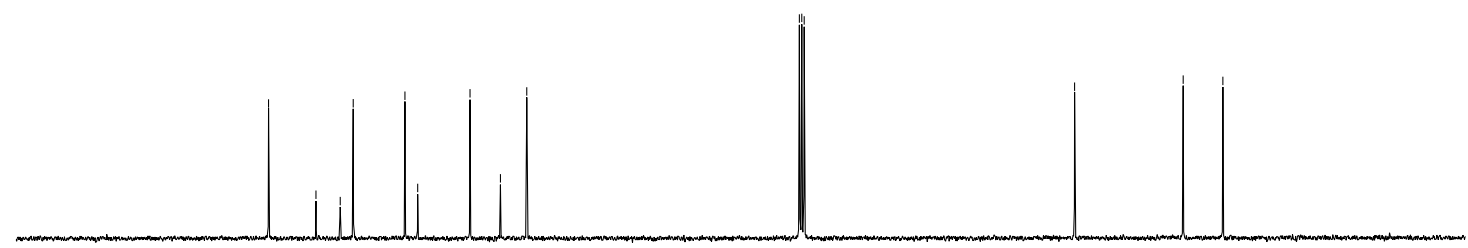

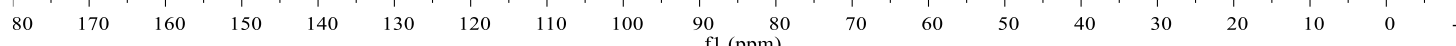


${ }^{1} \mathrm{H}$ NMR $\left(400 \mathrm{MHz}, \mathrm{CDCl}_{3}\right)$ spectrum of $\mathbf{6 f}$

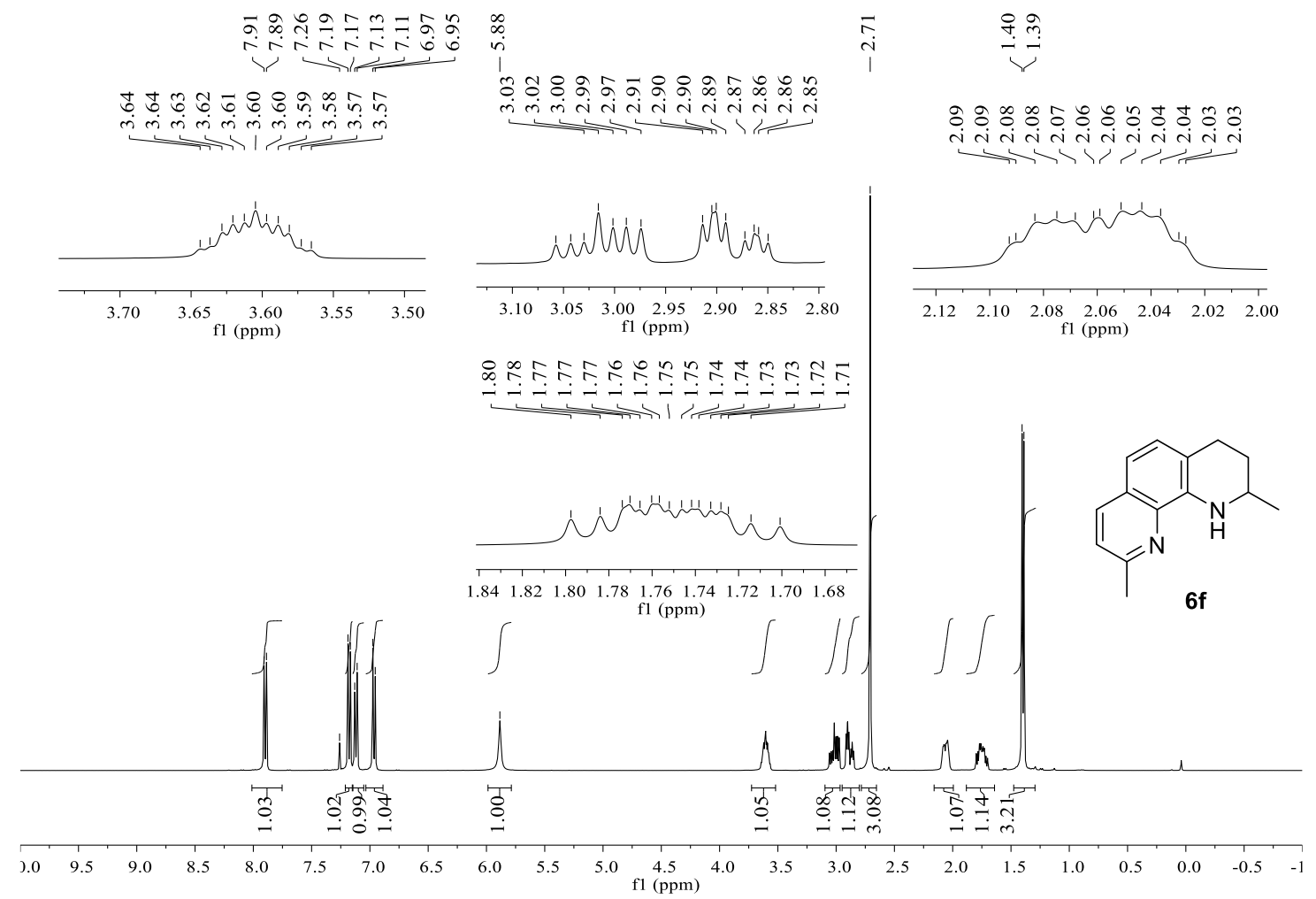

${ }^{13} \mathrm{C}\left\{{ }^{1} \mathrm{H}\right\}$ NMR $\left(101 \mathrm{MHz}, \mathrm{CDCl}_{3}\right)$ spectrum of $\mathbf{6} \mathbf{f}$

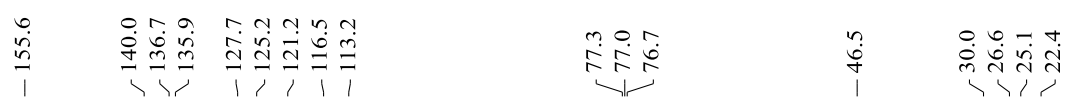
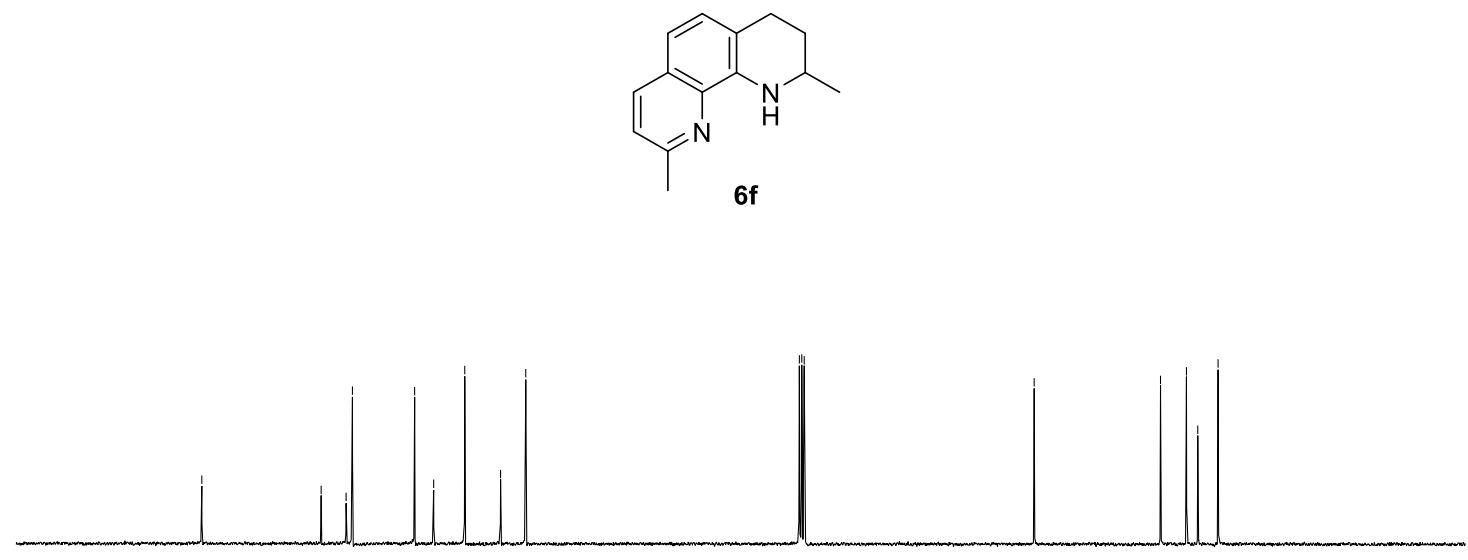

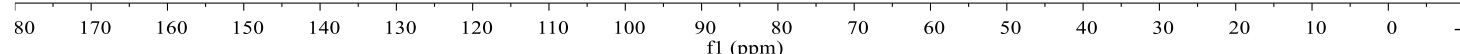


${ }^{1} \mathrm{H}$ NMR (400 MHz, $\mathrm{CDCl}_{3}$ ) spectrum of $\mathbf{6 g}$

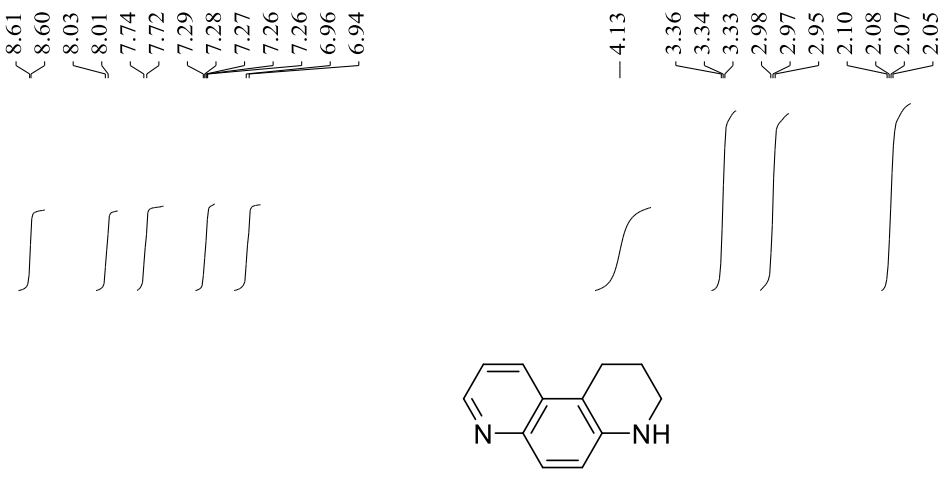

$6 \mathrm{~g}$

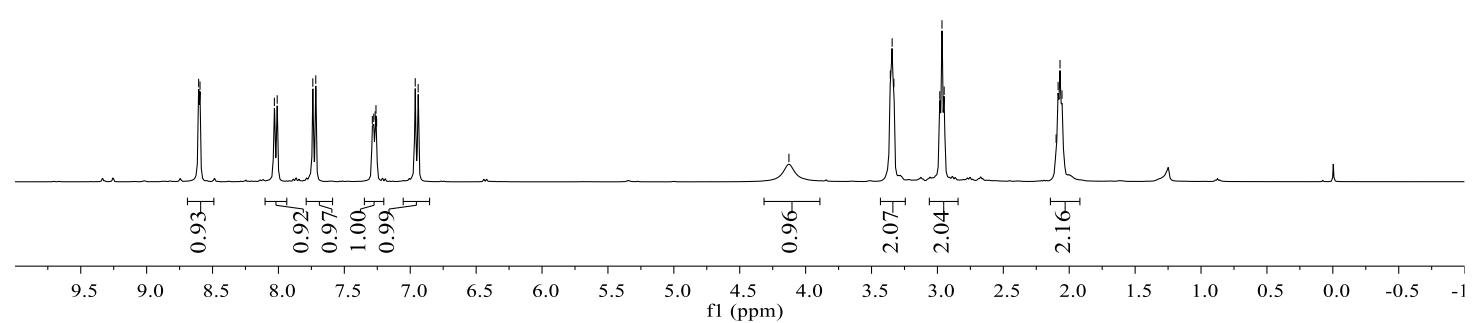

${ }^{13} \mathrm{C}\left\{{ }^{1} \mathrm{H}\right\} \mathrm{NMR}\left(101 \mathrm{MHz}, \mathrm{CDCl}_{3}\right)$ spectrum of $\mathbf{6 g}$

方莳

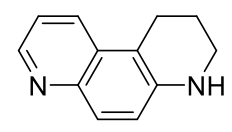

$6 \mathrm{~g}$

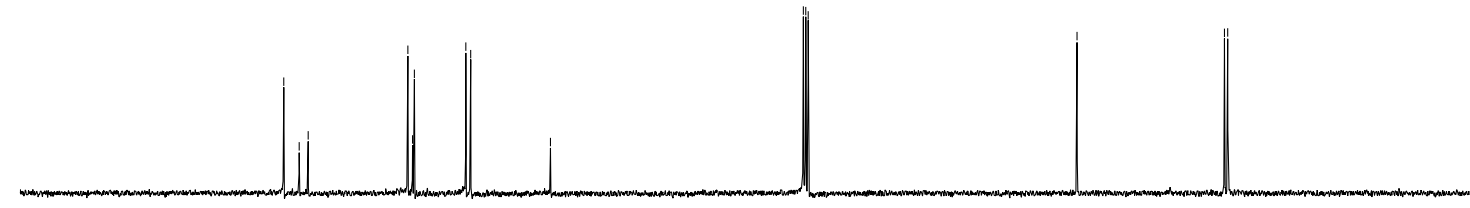

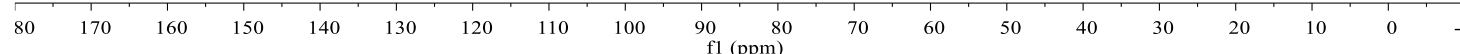


${ }^{1} \mathrm{H}$ NMR $\left(400 \mathrm{MHz}, \mathrm{CDCl}_{3}\right)$ spectrum of $\mathbf{6} \mathbf{h}$

$$
\stackrel{\substack{++}}{i} \underset{i}{i}
$$

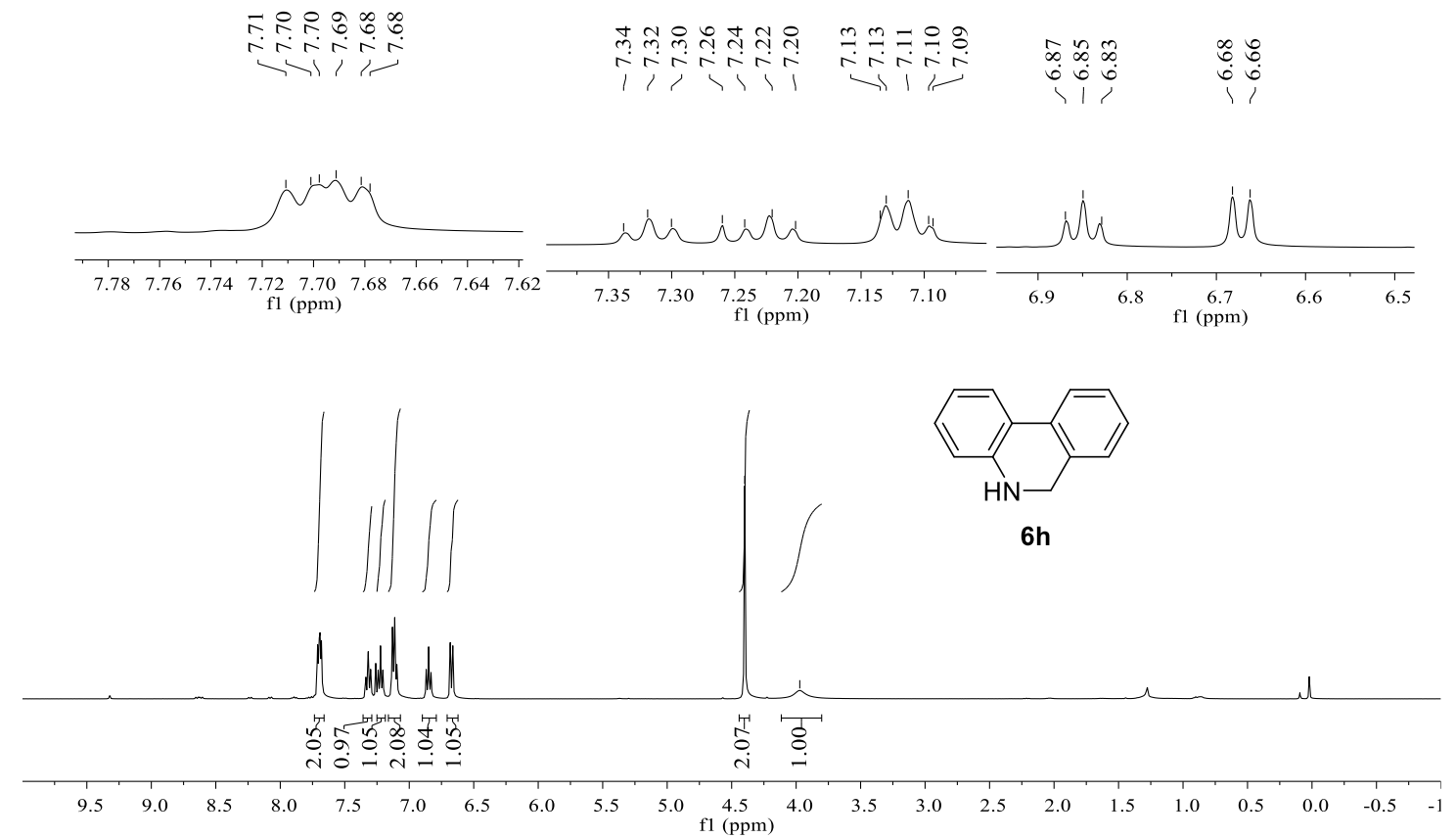

${ }^{13} \mathrm{C}\left\{{ }^{1} \mathrm{H}\right\}$ NMR $\left(101 \mathrm{MHz}, \mathrm{CDCl}_{3}\right)$ spectrum of $\mathbf{6 h}$
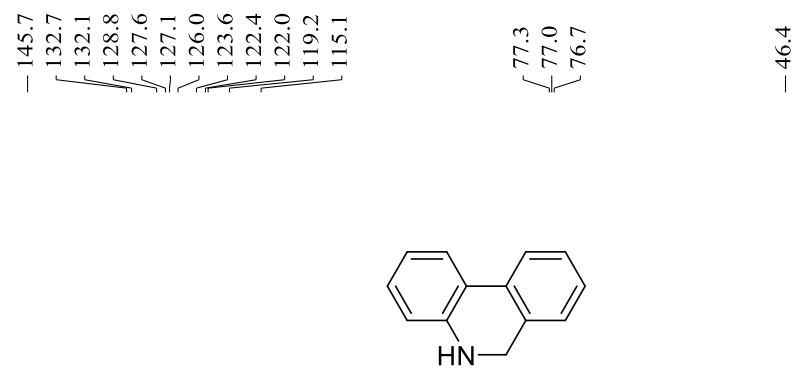

6h

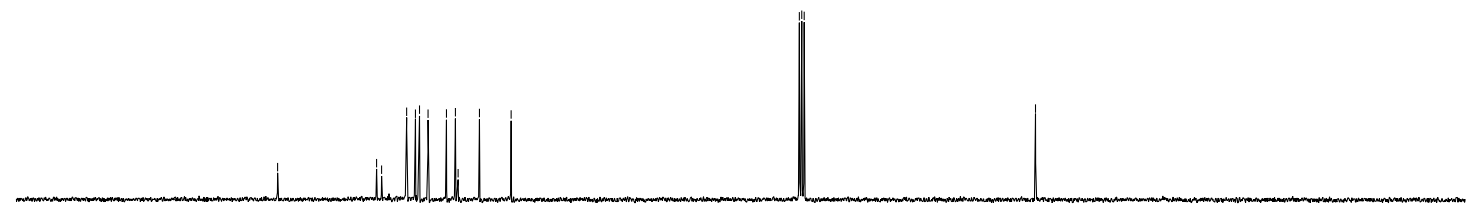

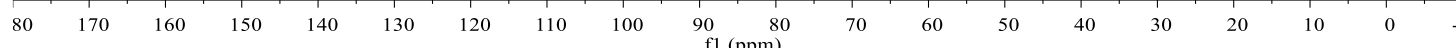


${ }^{1} \mathrm{H}$ NMR $\left(400 \mathrm{MHz}, \mathrm{CDCl}_{3}\right)$ spectrum of $\mathbf{6 i}$
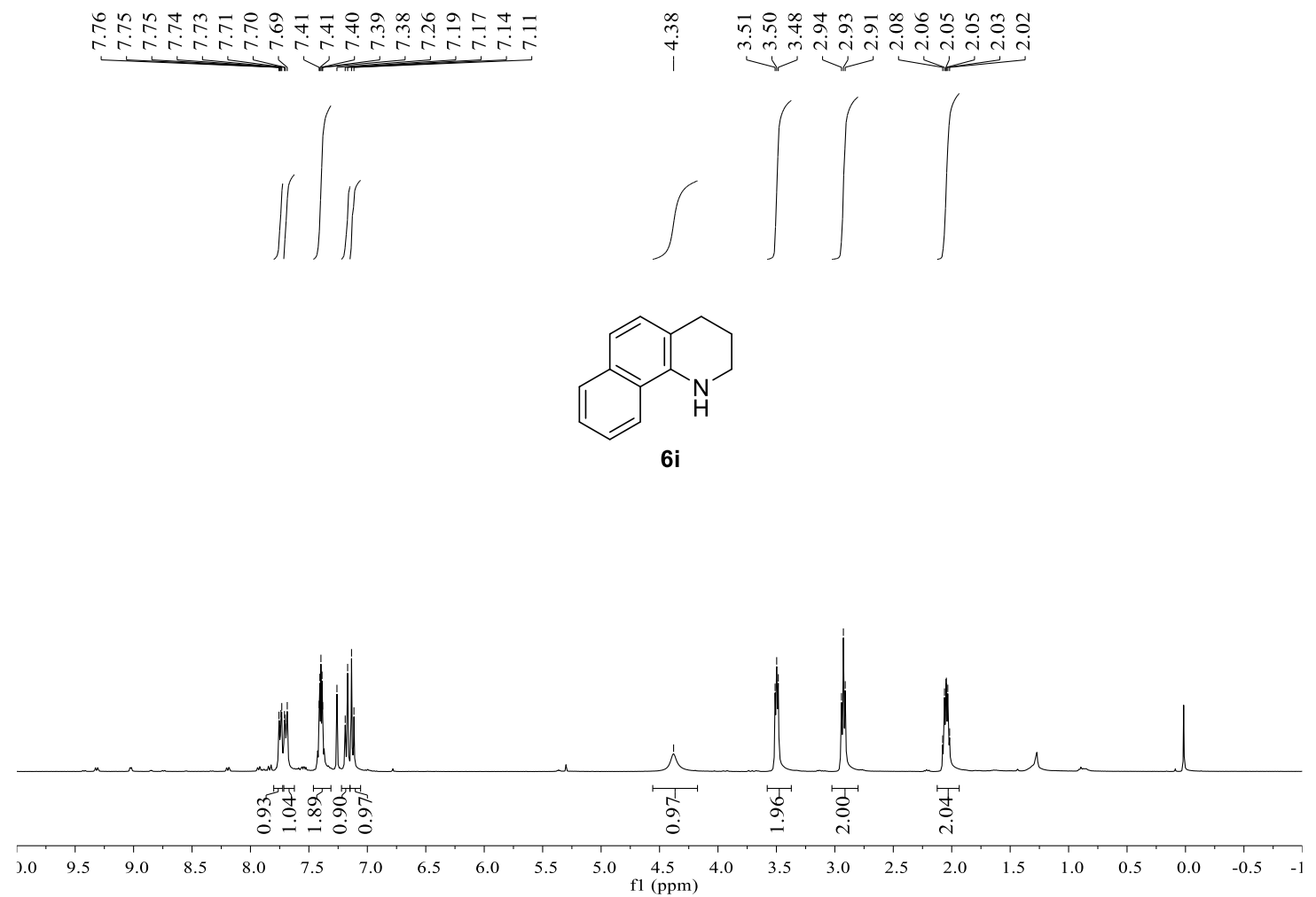

${ }^{13} \mathrm{C}\left\{{ }^{1} \mathrm{H}\right\}$ NMR $\left(101 \mathrm{MHz}, \mathrm{CDCl}_{3}\right)$ spectrum of $\mathbf{6 i}$

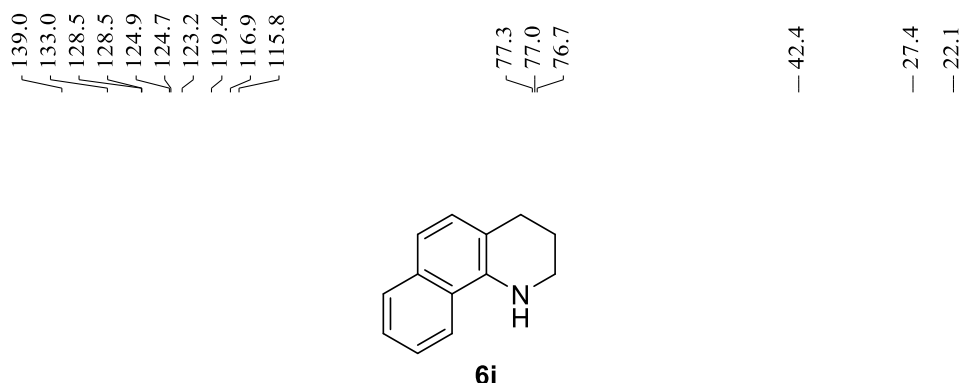

6i

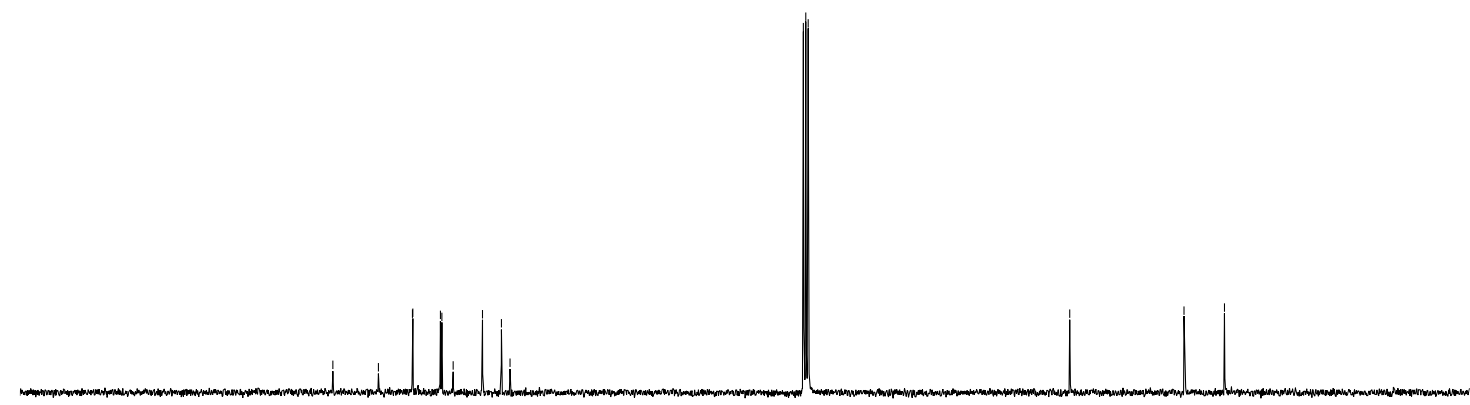

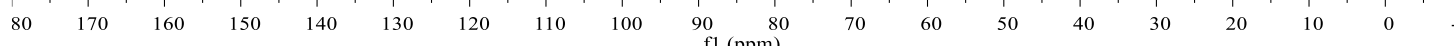


${ }^{1} \mathrm{H}$ NMR $\left(400 \mathrm{MHz}, \mathrm{CDCl}_{3}\right)$ spectrum of $\mathbf{6 j}$
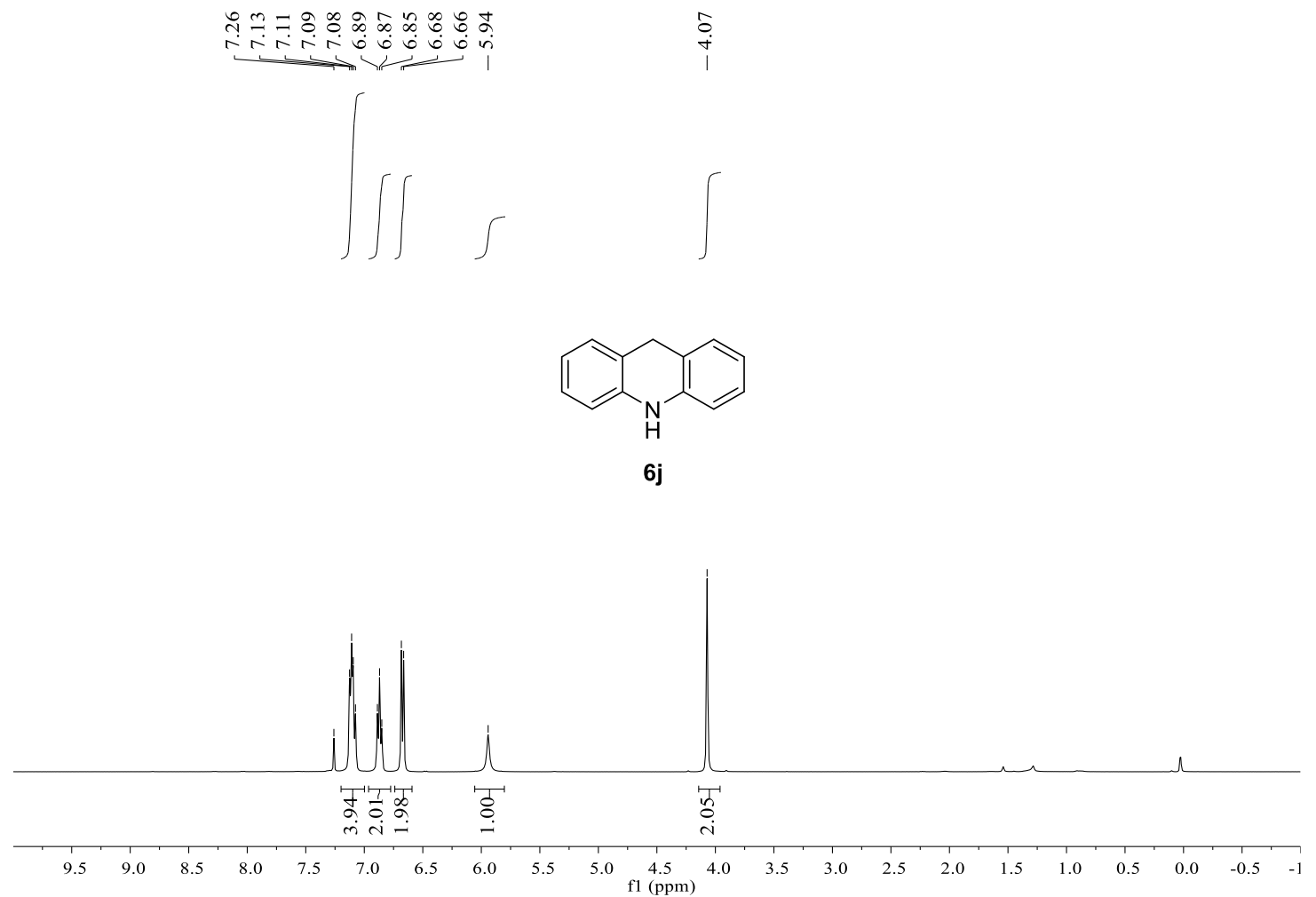

${ }^{13} \mathrm{C}\left\{{ }^{1} \mathrm{H}\right\}$ NMR $\left(101 \mathrm{MHz}, \mathrm{CDCl}_{3}\right)$ spectrum of $\mathbf{6 j}$
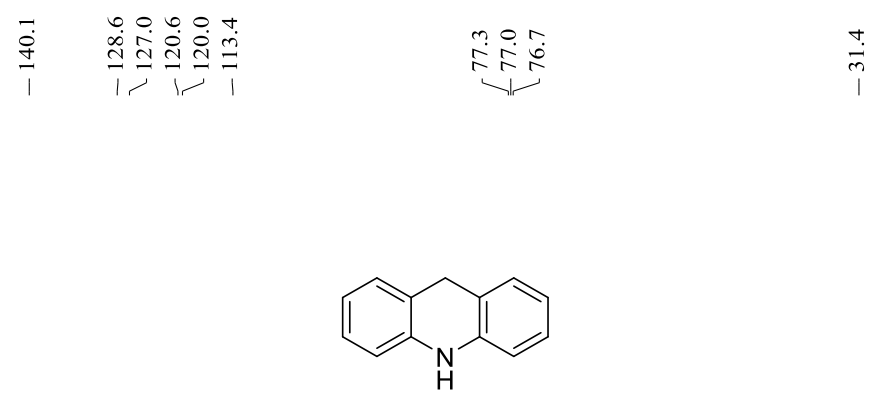

6j

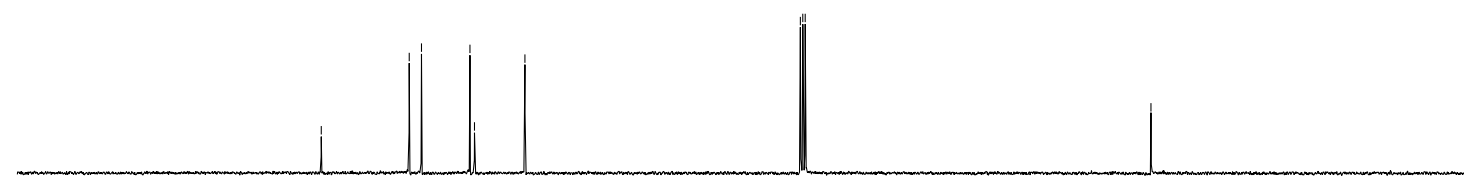

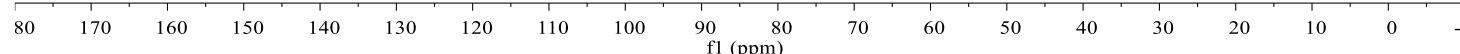


${ }^{1} \mathrm{H}$ NMR $\left(400 \mathrm{MHz}, \mathrm{CDCl}_{3}\right)$ spectrum of $\mathbf{6 k}$

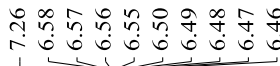

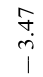

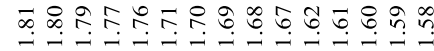

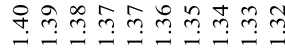<smiles>c1ccc2c(c1)NC1CCCCC1N2</smiles>

6k
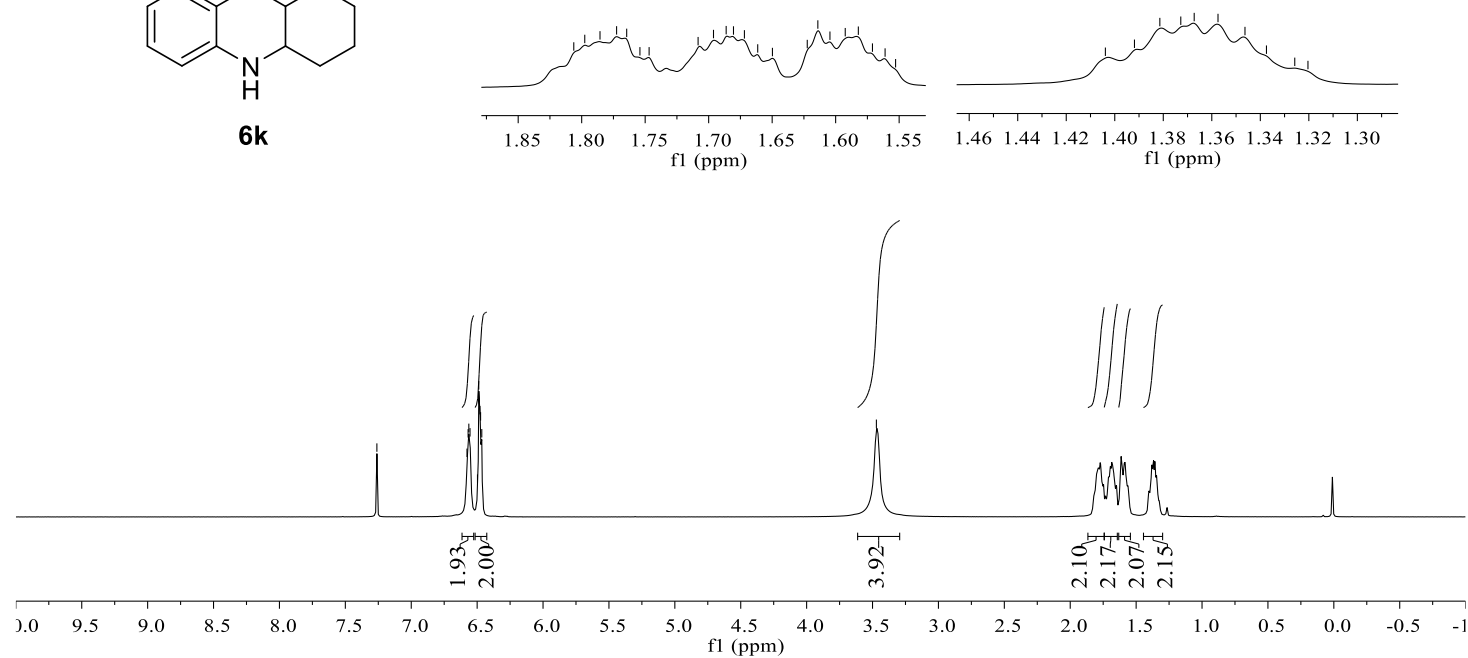

${ }^{13} \mathrm{C}\left\{{ }^{1} \mathrm{H}\right\}$ NMR $\left(101 \mathrm{MHz}, \mathrm{CDCl}_{3}\right)$ spectrum of $\mathbf{6 k}$

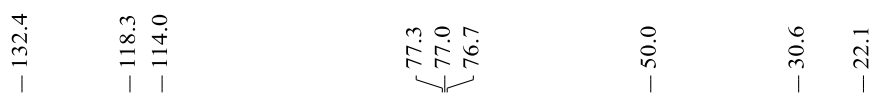<smiles>N#CC1Nc2ccccc2NC2CCCCC12</smiles>

6k

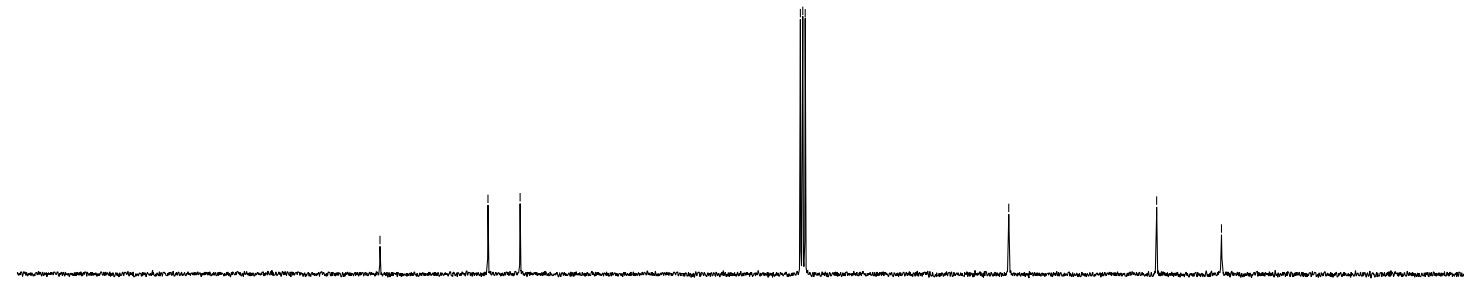

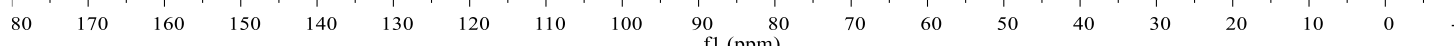


${ }^{1} \mathrm{H}$ NMR $\left(400 \mathrm{MHz}, \mathrm{CDCl}_{3}\right)$ spectrum of $\mathbf{6 n}$

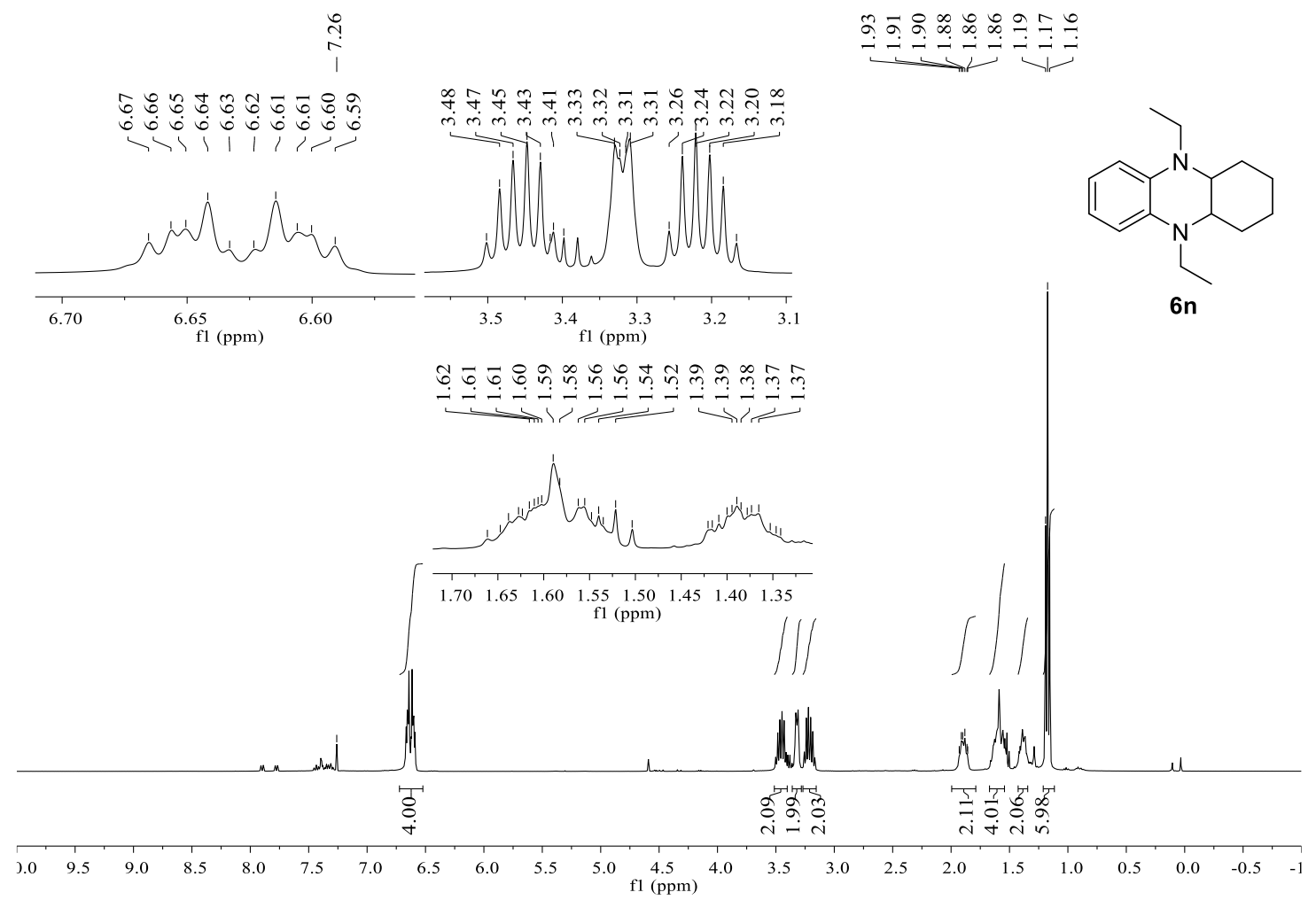

${ }^{13} \mathrm{C}\left\{{ }^{1} \mathrm{H}\right\}$ NMR $\left(101 \mathrm{MHz}, \mathrm{CDCl}_{3}\right)$ spectrum of $\mathbf{6 n}$

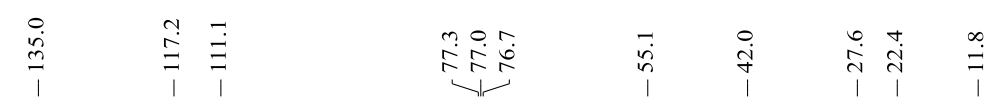<smiles>CCN1c2ccccc2N(CC)C2CCCCC21</smiles>

$6 n$

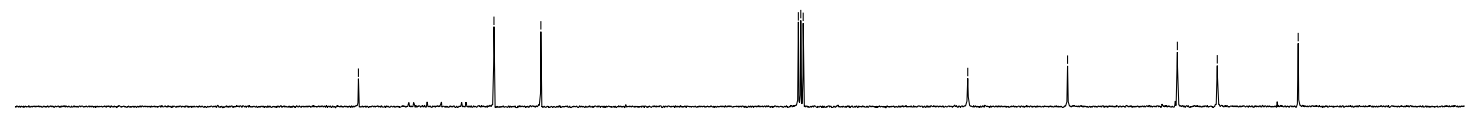

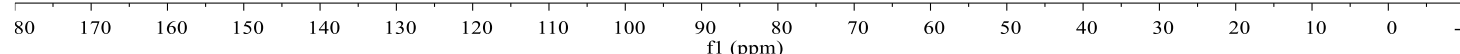


${ }^{1} \mathrm{H}$ NMR (400 MHz, $\mathrm{CDCl}_{3}$ ) spectrum of $\mathbf{L - 6}$

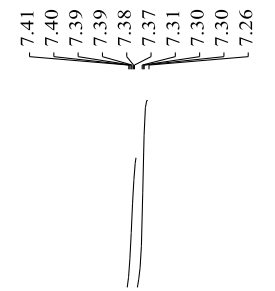

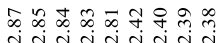

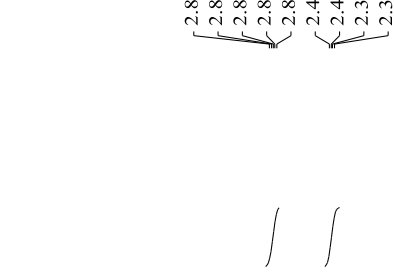

$\mathrm{Ph}_{2} \mathrm{P} \sim{ }_{\mathrm{L}-6}^{\mathrm{H}} \sim_{\mathrm{PPh}_{2}}^{\mathrm{H}}$

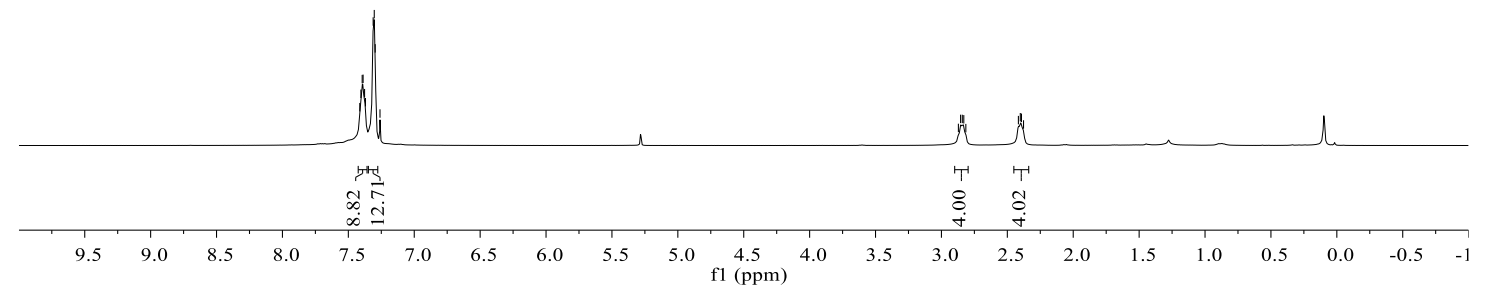

${ }^{13} \mathrm{C}\left\{{ }^{1} \mathrm{H}\right\}$ NMR $\left(101 \mathrm{MHz}, \mathrm{CDCl}_{3}\right)$ spectrum of $\mathbf{L}-\mathbf{6}$
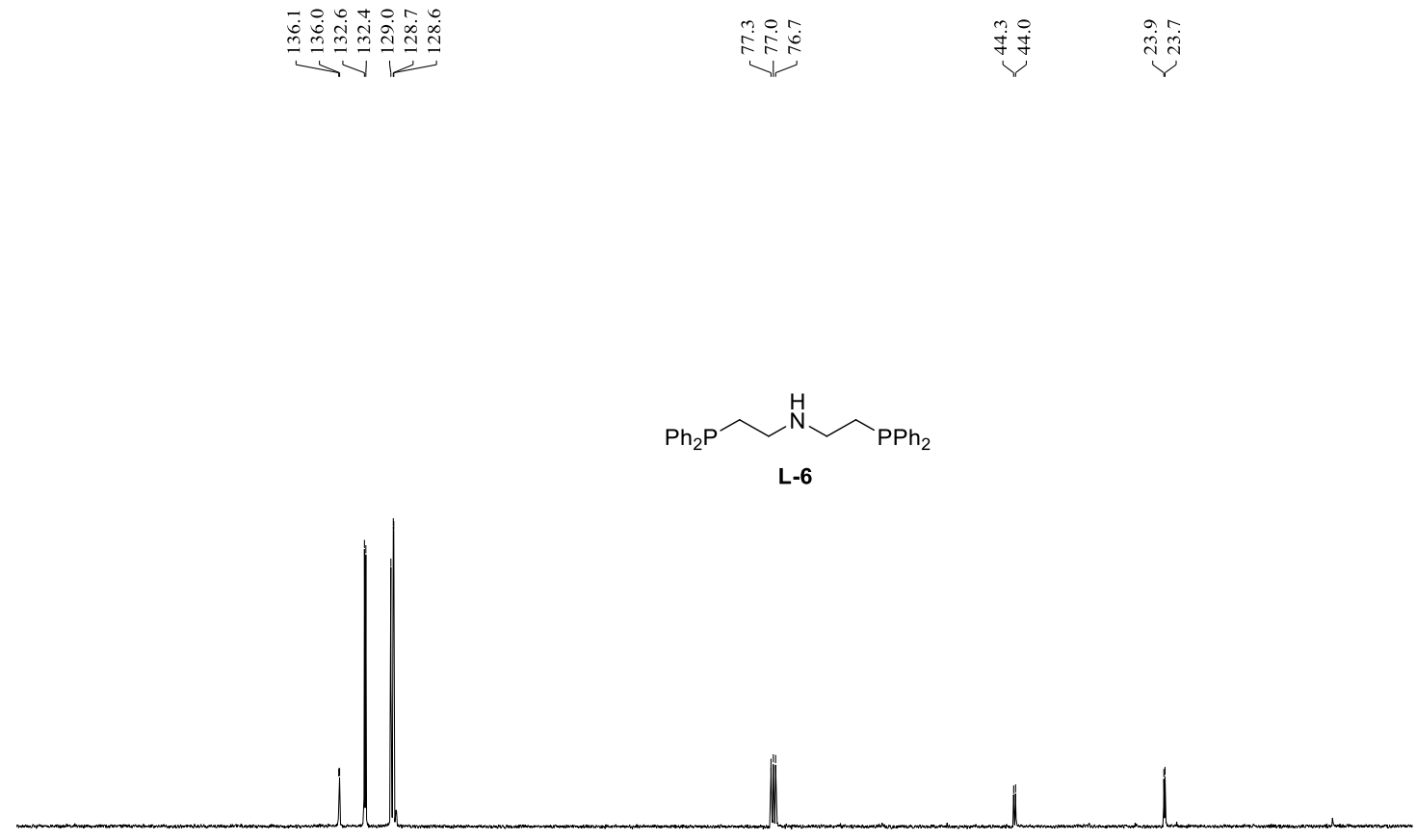

$\begin{array}{llllllllll}80 & 170 & 160 & 150 & 140 & 130 & 120 & 110 & 100 & 90 \quad 80 \\ \mathrm{f} 1(\mathrm{ppm})\end{array}$ 
${ }^{31} \mathrm{P}\left\{{ }^{1} \mathrm{H}\right\}$ NMR $\left(162 \mathrm{MHz}, \mathrm{CDCl}_{3}\right)$ spectrum of $\mathbf{L}-\mathbf{6}$

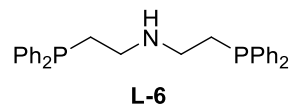

L-6

$\begin{array}{lllllllllllllllllllllllllllllllllll}140 & 130 & 120 & 110 & 100 & 90 & 80 & 70 & 60 & 50 & 40 & 30 & 20 & 10 & 0 & -10 & -20 & -30 & -40 & -50 & -60 & -70 & -80 & -90 & -100 & -110 & -120 & -130 & -140\end{array}$

${ }^{1} \mathrm{H}$ NMR (400 MHz, $\mathrm{CDCl}_{3}$ ) spectrum of $\mathbf{L - 2}$

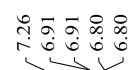

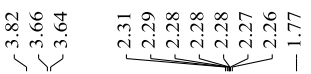

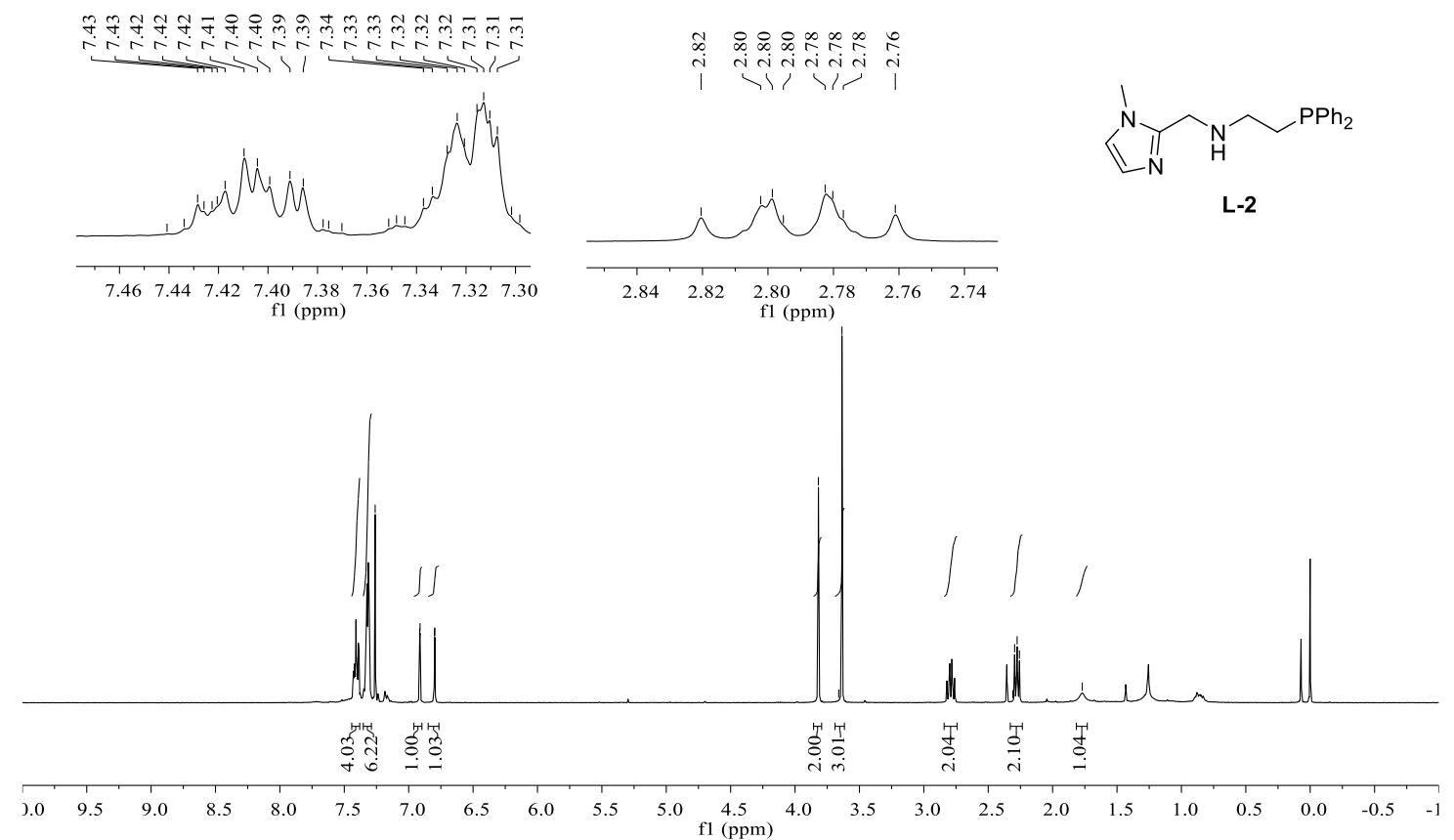


${ }^{13} \mathrm{C}\left\{{ }^{1} \mathrm{H}\right\}$ NMR $\left(101 \mathrm{MHz}, \mathrm{CDCl}_{3}\right)$ spectrum of $\mathbf{L}-\mathbf{2}$

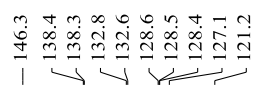

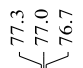

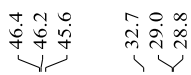

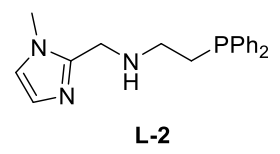

L-2

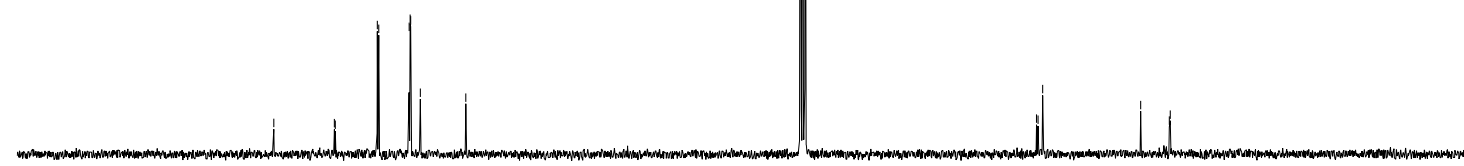

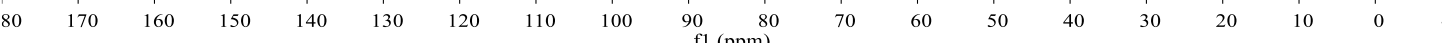

${ }^{31} \mathrm{P}\left\{{ }^{1} \mathrm{H}\right\}$ NMR $\left(162 \mathrm{MHz}, \mathrm{CDCl}_{3}\right)$ spectrum of $\mathbf{L}-\mathbf{2}$

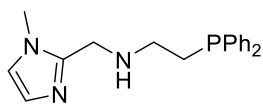

L-2

$\begin{array}{lllllllllllllllllllllllllllll}140 & 130 & 120 & 110 & 100 & 90 & 80 & 70 & 60 & 50 & 40 & 30 & 20 & 10 & 0 & -10 & -20 & -30 & -40 & -50 & -60 & -70 & -80 & -90 & -100 & -110 & -120 & -130 & -140\end{array}$ 
${ }^{1} \mathrm{H}$ NMR (400 MHz, DMSO- $d_{6}$ ) spectrum of Mn-2

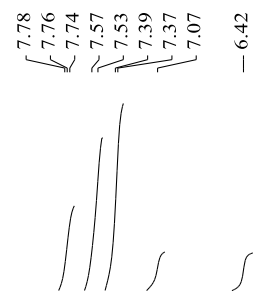

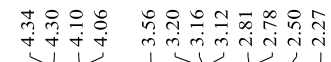
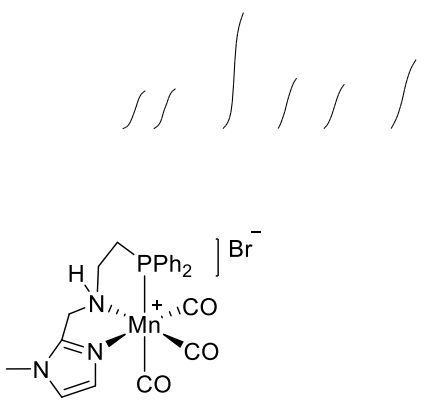

$[\mathrm{Mn}]-2$

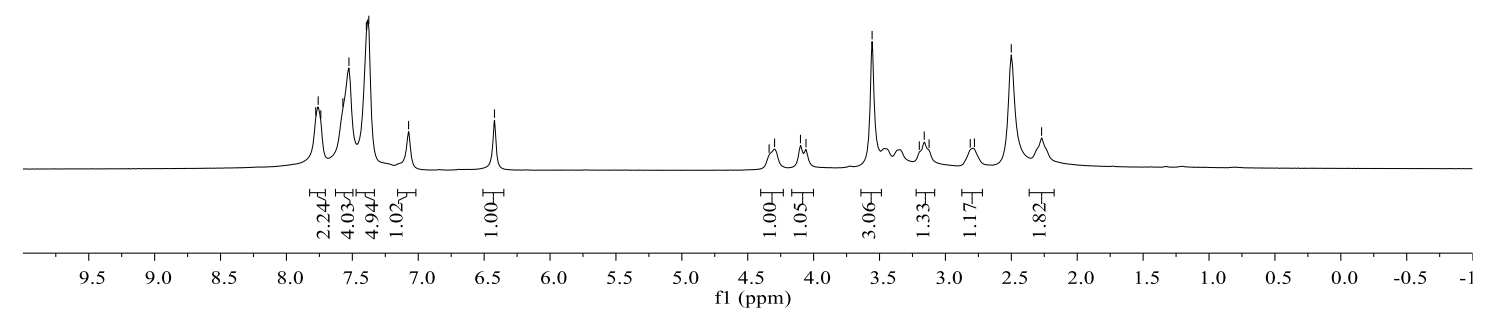

${ }^{13} \mathrm{C}\left\{{ }^{1} \mathrm{H}\right\}$ NMR (101 MHz, DMSO- $\left.d_{6}\right)$ spectrum of Mn-2

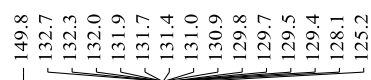

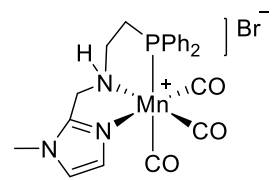

$[\mathrm{Mn}]-2$

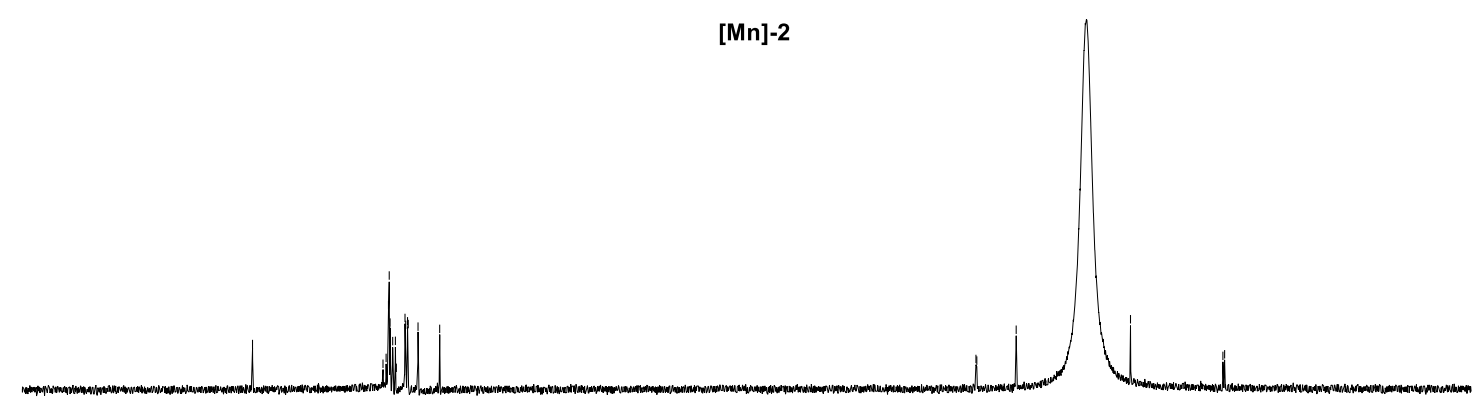

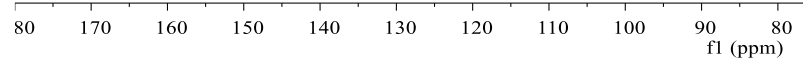


${ }^{31} \mathrm{P}\left\{{ }^{1} \mathrm{H}\right\}$ NMR $\left(162 \mathrm{MHz}, \mathrm{DMSO}-d_{6}\right)$ spectrum of Mn-2

$\hat{a}$

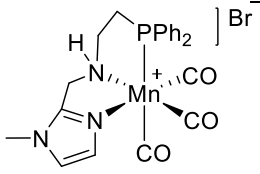

[Mn]-2

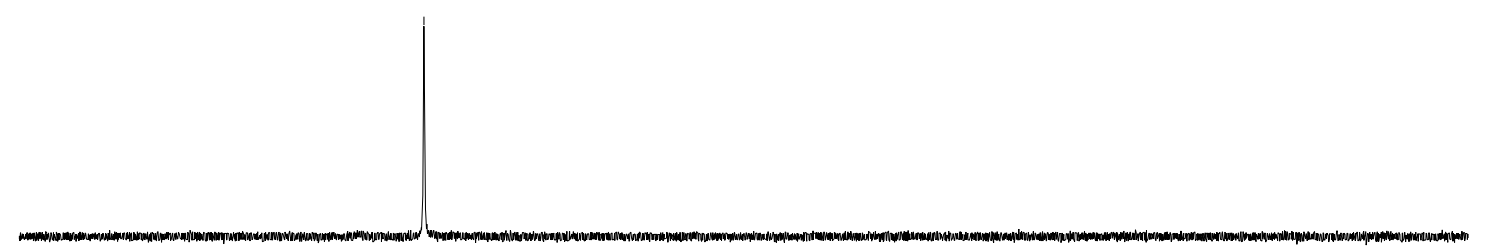

$\begin{array}{llllllllllllllllllllllllllllllllllllll}140 & 130 & 120 & 110 & 100 & 90 & 80 & 70 & 60 & 50 & 40 & 30 & 20 & 10 & 0 & -10 & -20 & -30 & -40 & -50 & -60 & -70 & -80 & -90 & -100 & -110 & -120 & -130 & -140\end{array}$

${ }^{1} \mathrm{H}$ NMR $\left(400 \mathrm{MHz}, \mathrm{CD}_{2} \mathrm{Cl}_{2}\right)$ spectrum of Mn-6
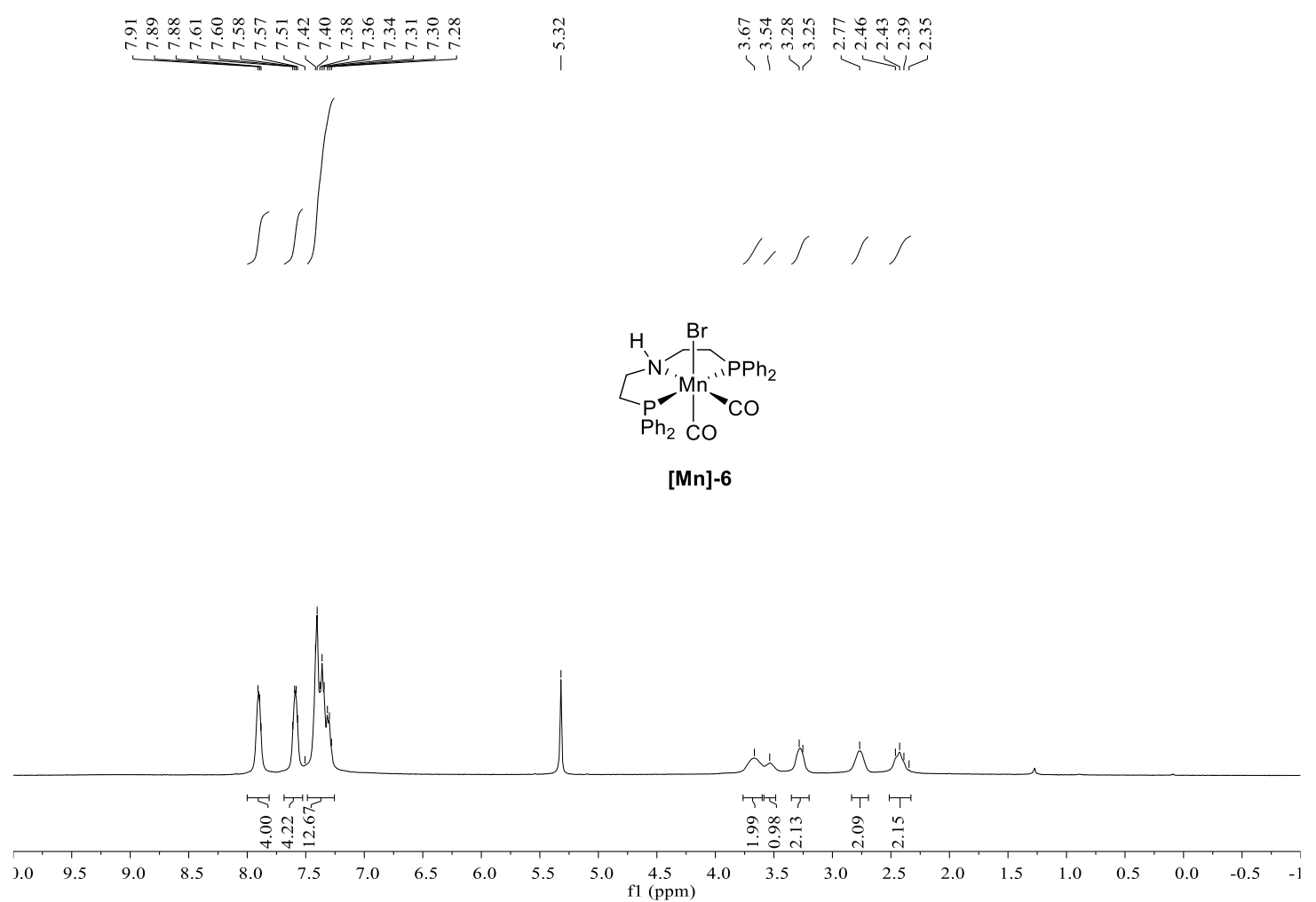
${ }^{13} \mathrm{C}\left\{{ }^{1} \mathrm{H}\right\}$ NMR (101 MHz, $\left.\mathrm{CD}_{2} \mathrm{Cl}_{2}\right)$ spectrum of Mn-6

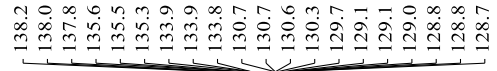

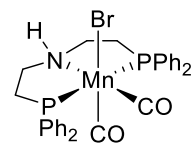

[Mn]-6
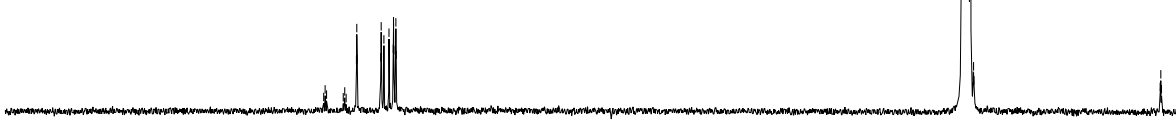

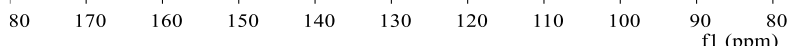

${ }^{31} \mathrm{P}\left\{{ }^{1} \mathrm{H}\right\}$ NMR $\left(162 \mathrm{MHz}, \mathrm{CD}_{2} \mathrm{Cl}_{2}\right)$ spectrum of Mn-6

$$
\begin{aligned}
& 3 \\
& 8 \\
& 0 \\
& 1
\end{aligned}
$$

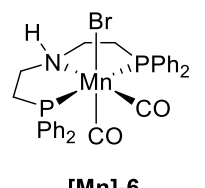

[Mn]-6 
${ }^{1} \mathrm{H}$ NMR (400 MHz, $\mathrm{C}_{6} \mathrm{D}_{6}$ ) spectrum of Mn-1a

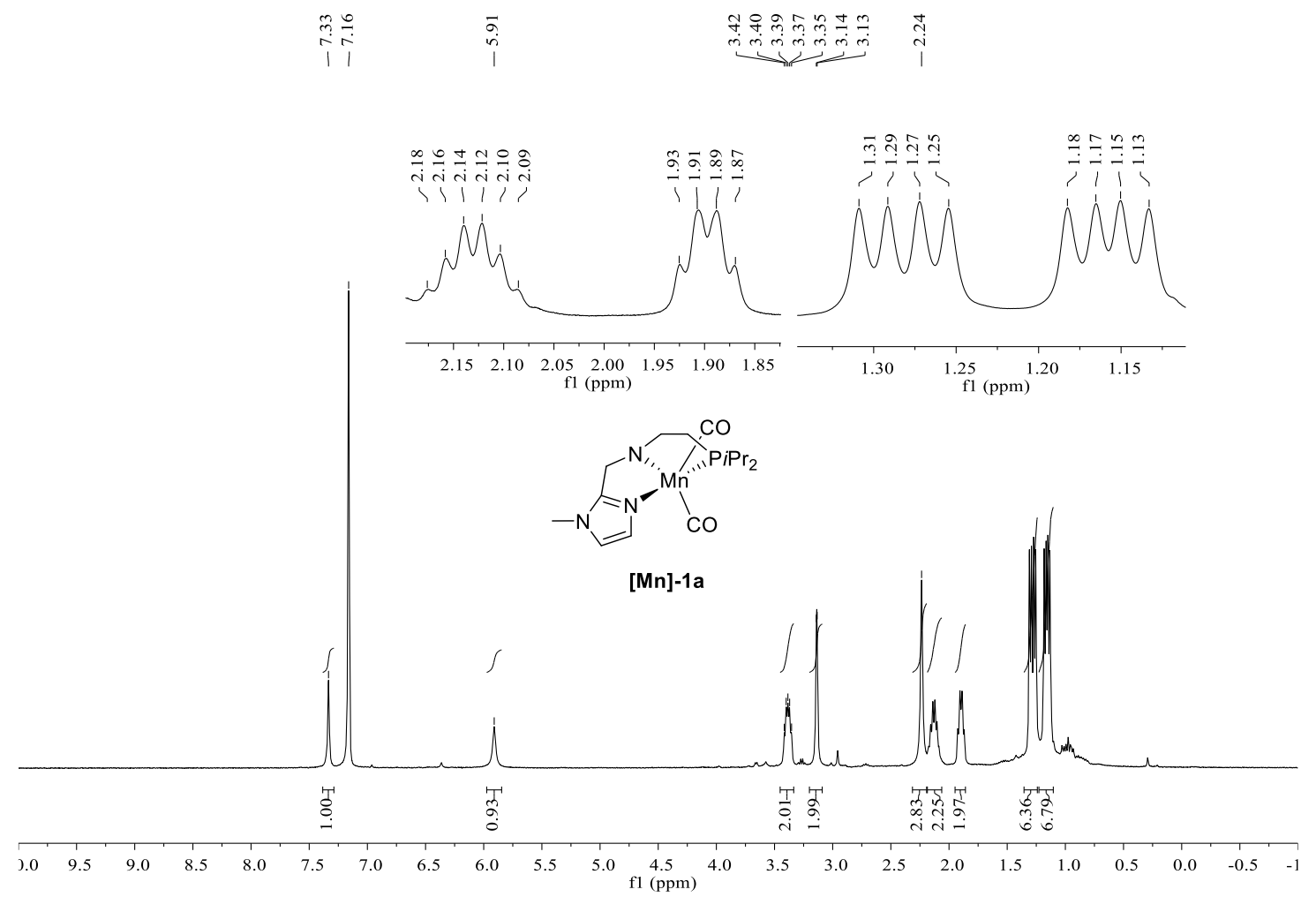

${ }^{13} \mathrm{C}\left\{{ }^{1} \mathrm{H}\right\}$ NMR (101 MHz, $\left.\mathrm{C}_{6} \mathrm{D}_{6}\right)$ spectrum of Mn-1a

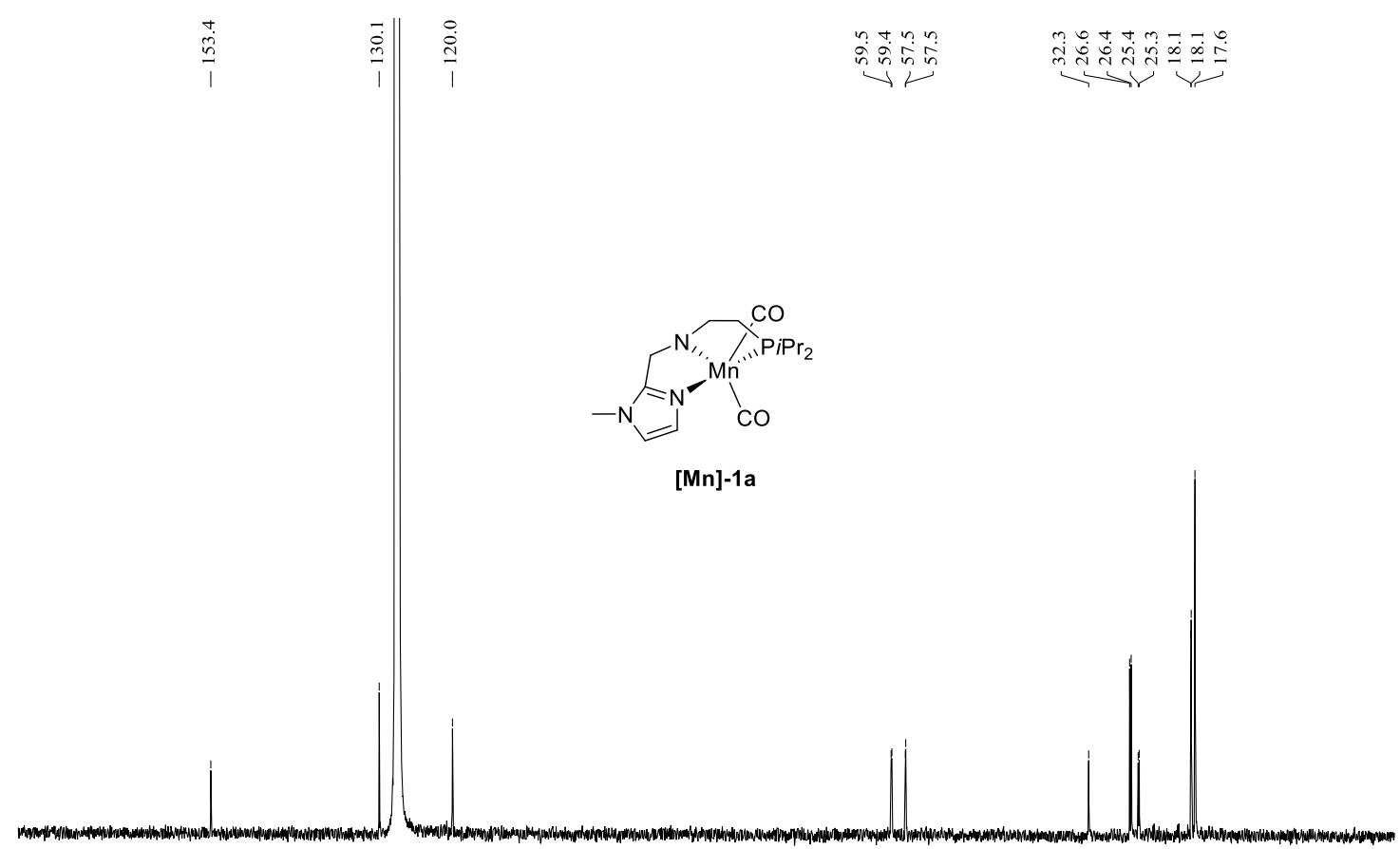

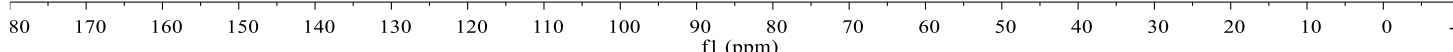


${ }^{31} \mathrm{P}\left\{{ }^{1} \mathrm{H}\right\}$ NMR $\left(162 \mathrm{MHz}, \mathrm{C}_{6} \mathrm{D}_{6}\right)$ spectrum of Mn-1a

$$
\stackrel{\substack{n \\ \stackrel{\sim}{J} \\ \hline}}{1}
$$

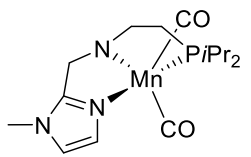

[Mn]-1a

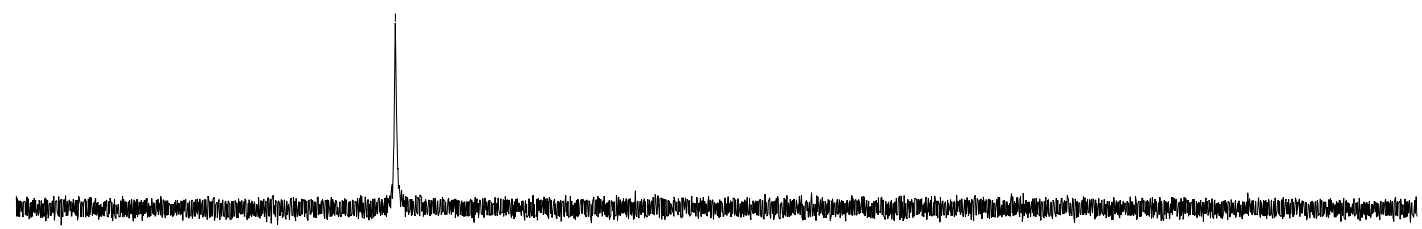

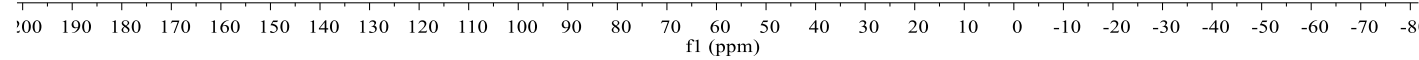

${ }^{1} \mathrm{H}$ NMR (400 MHz, $\mathrm{C}_{6} \mathrm{D}_{6}$ ) spectrum of Mn-2a

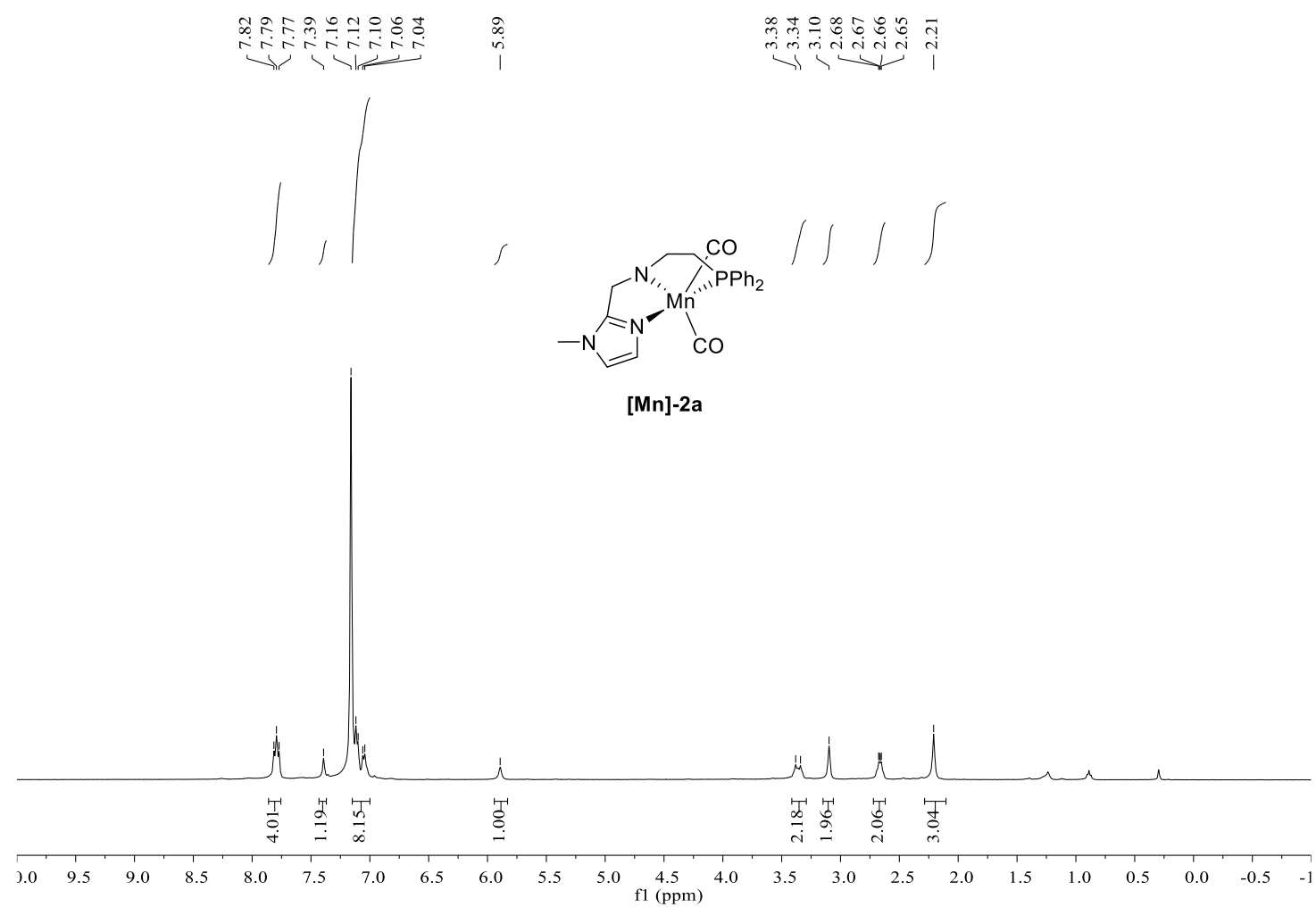


${ }^{13} \mathrm{C}\left\{{ }^{1} \mathrm{H}\right\}$ NMR (101 MHz, $\left.\mathrm{C}_{6} \mathrm{D}_{6}\right)$ spectrum of Mn-2a

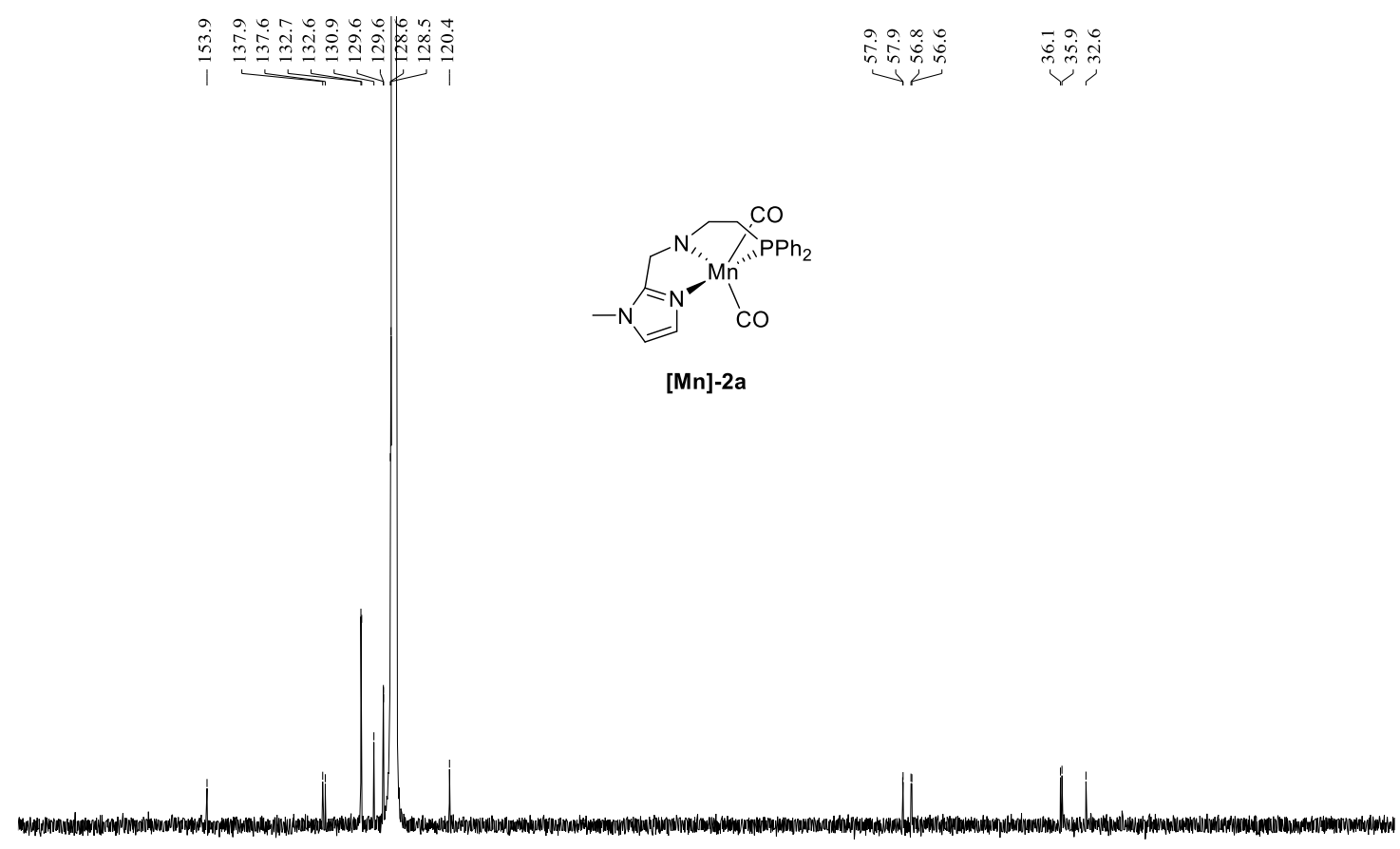

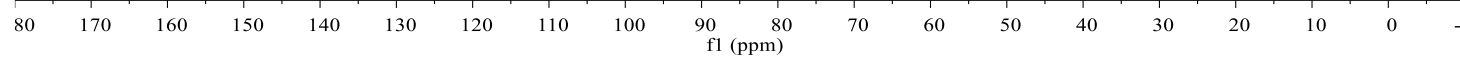

${ }^{31} \mathrm{P}\left\{{ }^{1} \mathrm{H}\right\}$ NMR (162 MHz, $\left.\mathrm{C}_{6} \mathrm{D}_{6}\right)$ spectrum of Mn-2a

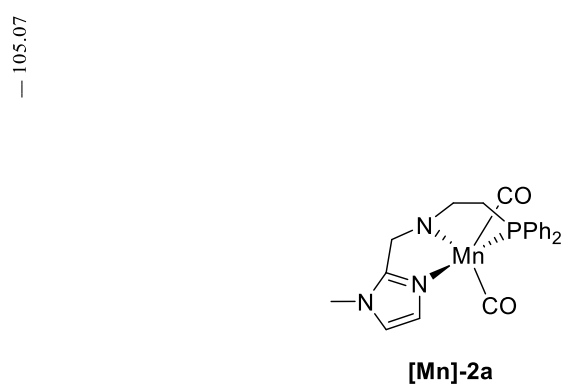

.

$\begin{array}{llllllllllllllllllllll}140 & 130 & 120 & 110 & 100 & 90 & 80 & 70 & 60 & 50 & 40 & 30 & 20 & 10 & 0 & -10 & -20 & -30 & -40 & -50 & -61\end{array}$ 
${ }^{1} \mathrm{H}$ NMR (400 MHz, $\mathrm{C}_{6} \mathrm{D}_{6}$ ) spectrum of $\mathbf{M n - 3 a}$

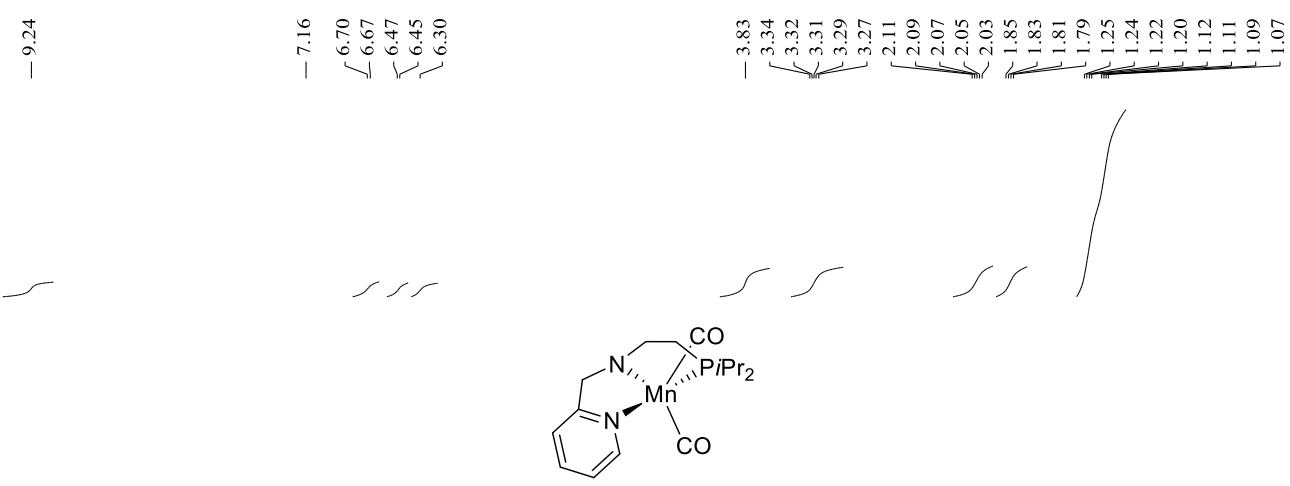

$[\mathrm{Mn}]-3 \mathrm{a}$

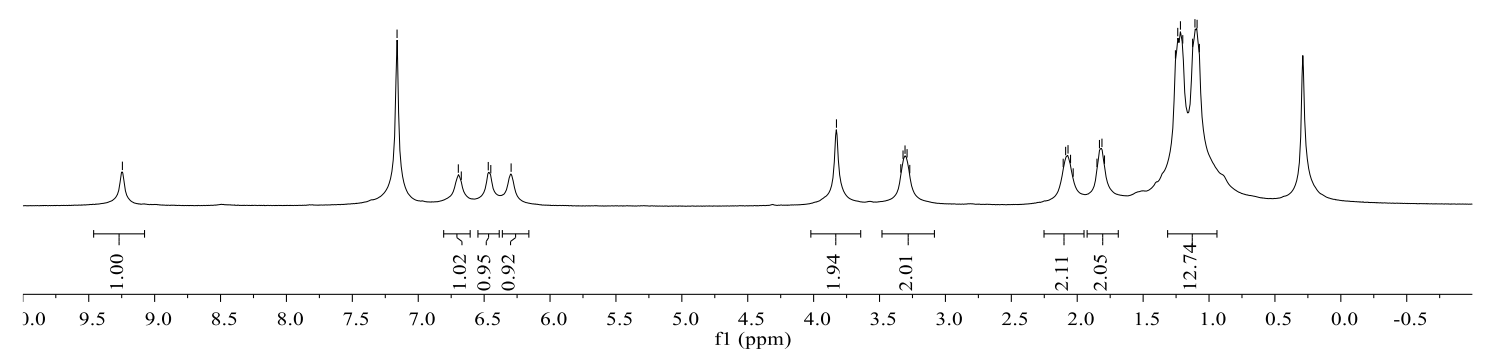

${ }^{13} \mathrm{C}\left\{{ }^{1} \mathrm{H}\right\}$ NMR $\left(101 \mathrm{MHz}, \mathrm{C}_{6} \mathrm{D}_{6}\right)$ spectrum of $\mathbf{M n}-\mathbf{3 a}$

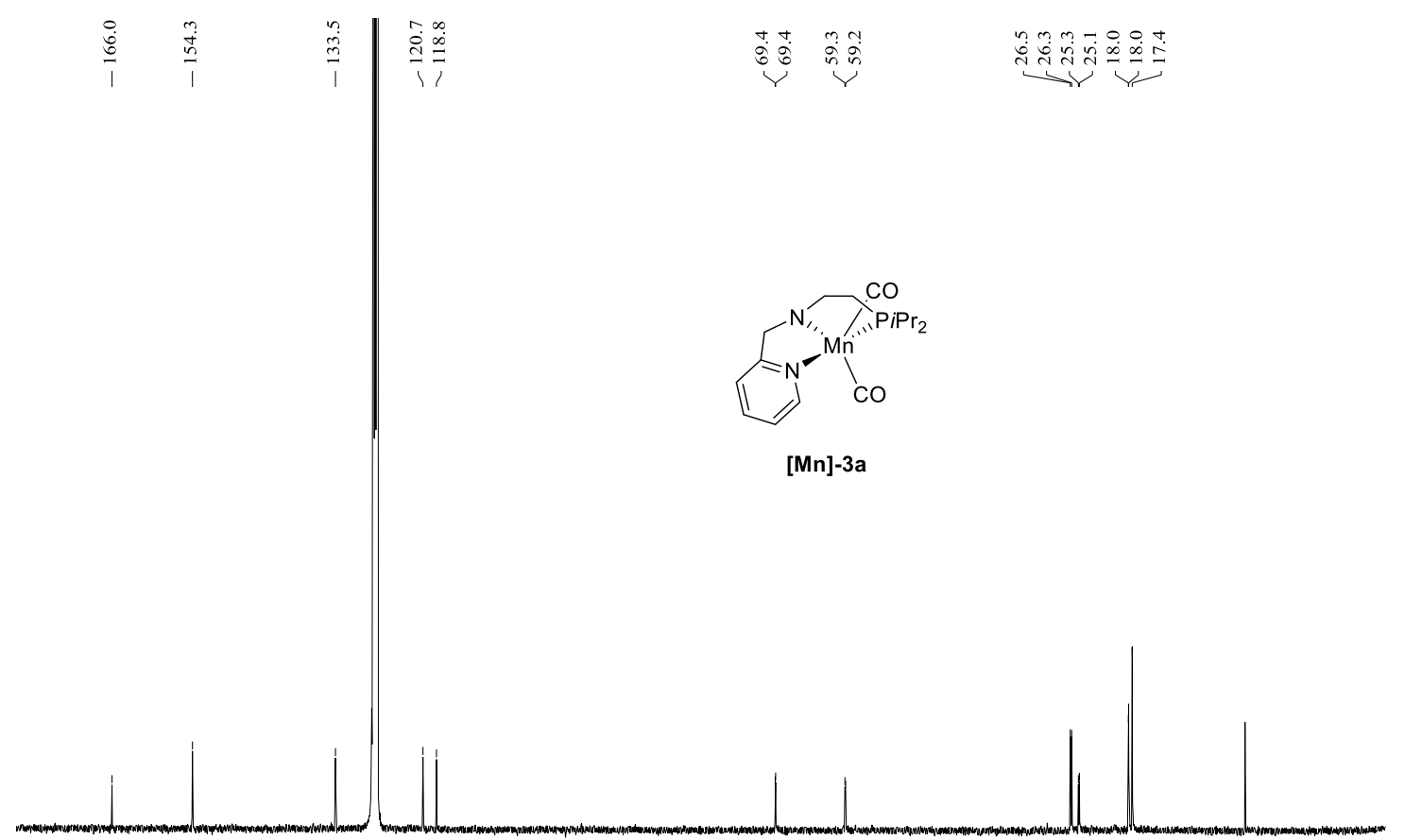

\begin{tabular}{llllllllllllllllllll}
\hline 80 & 170 & 160 & 150 & 140 & 130 & 120 & 110 & 100 & 90 & $\begin{array}{c}80 \\
\mathrm{f}(\mathrm{ppm})\end{array}$ & 70 & 60 & 50 & 40 & 30 & 20 & 10 & 0 & -10
\end{tabular} 
${ }^{31} \mathrm{P}\left\{{ }^{1} \mathrm{H}\right\}$ NMR (162 MHz, $\left.\mathrm{C}_{6} \mathrm{D}_{6}\right)$ spectrum of Mn-3a

$\stackrel{\circ}{\stackrel{0}{*}}$

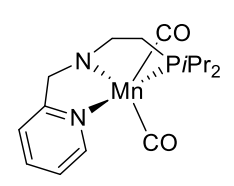

[Mn]-3a

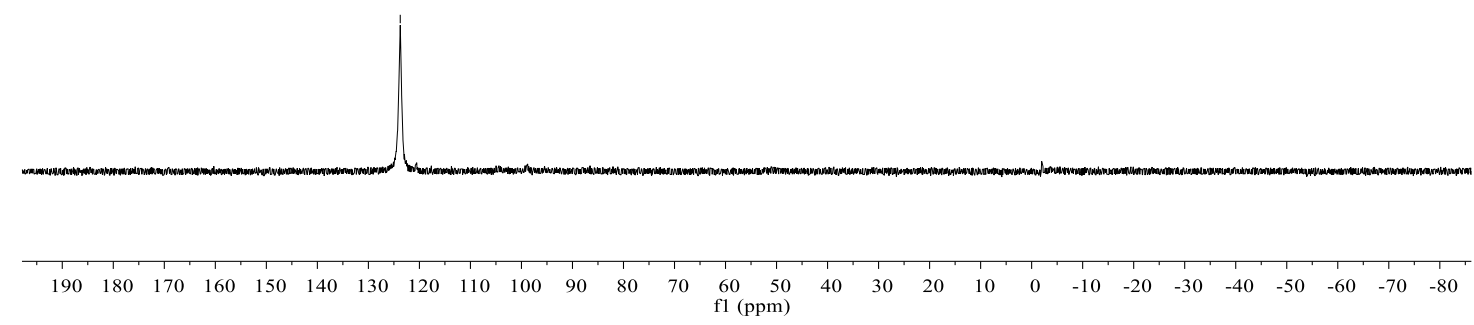

${ }^{1} \mathrm{H}$ NMR (400 MHz, $\mathrm{C}_{6} \mathrm{D}_{6}$ ) spectrum of $\mathbf{M n - 4 a}$

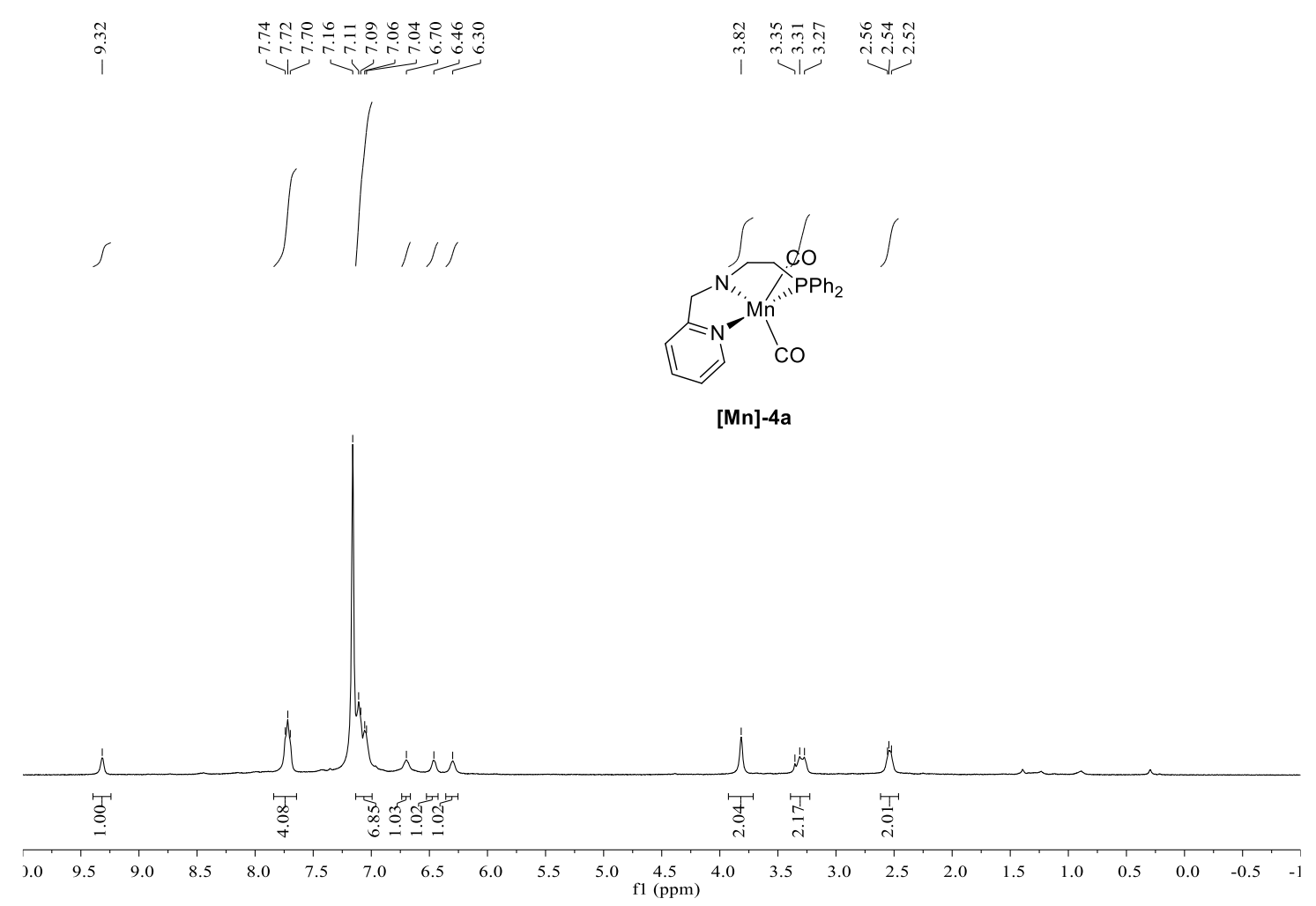


${ }^{13} \mathrm{C}\left\{{ }^{1} \mathrm{H}\right\}$ NMR (101 MHz, $\left.\mathrm{C}_{6} \mathrm{D}_{6}\right)$ spectrum of $\mathbf{M n}-\mathbf{4 a}$

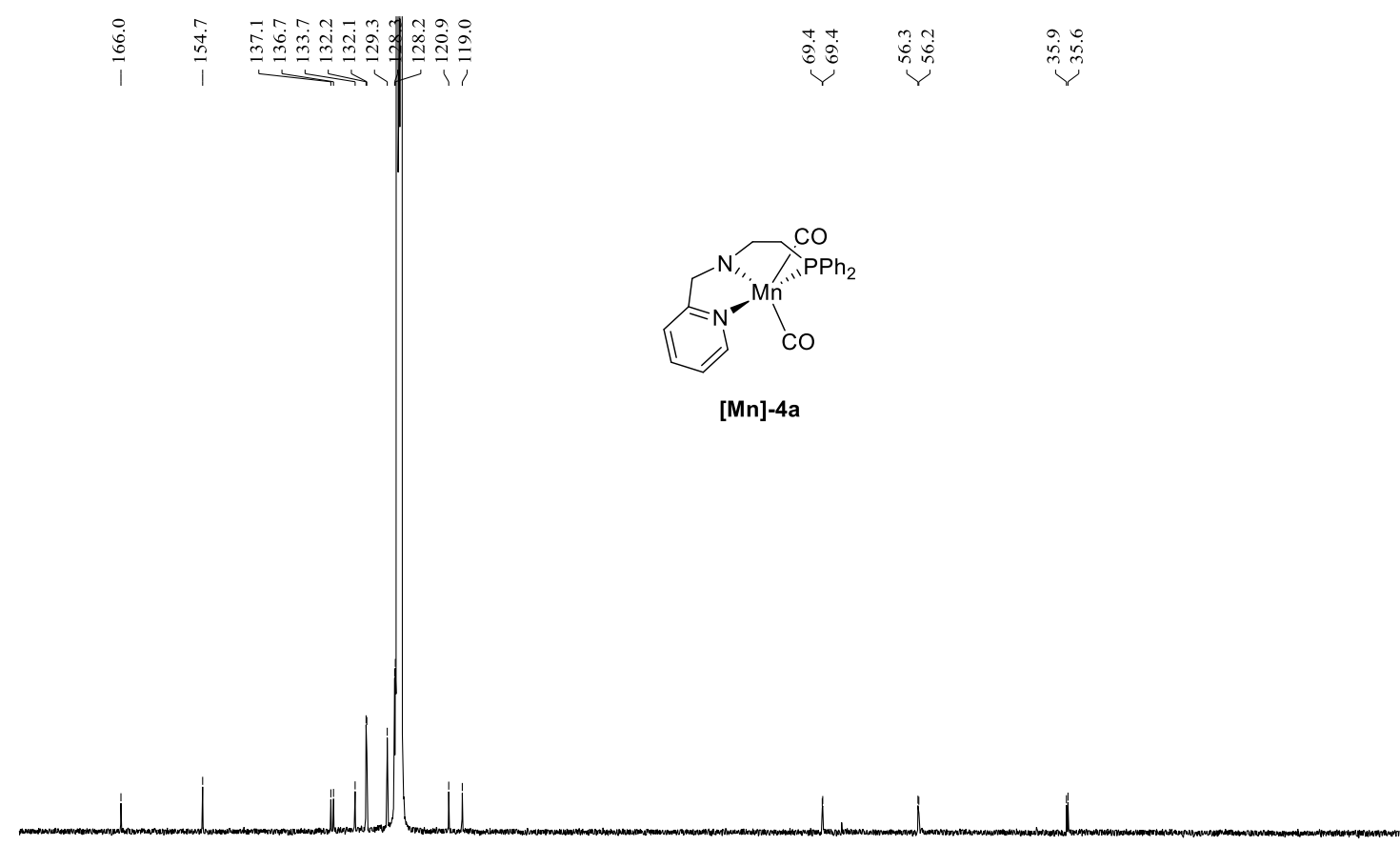

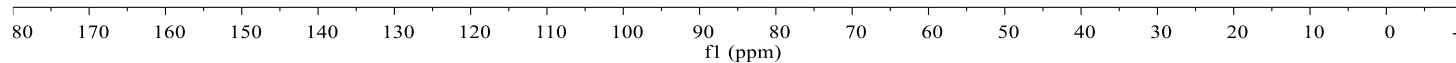

${ }^{31} \mathrm{P}\left\{{ }^{1} \mathrm{H}\right\}$ NMR $\left(162 \mathrm{MHz}, \mathrm{C}_{6} \mathrm{D}_{6}\right)$ spectrum of Mn-4a

$$
\stackrel{\substack{+\stackrel{0}{0}}}{1}
$$

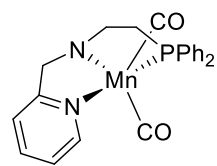

[Mn]-4a 
${ }^{1} \mathrm{H}$ NMR (400 MHz, $\mathrm{C}_{6} \mathrm{D}_{6}$ ) spectrum of Mn-6a
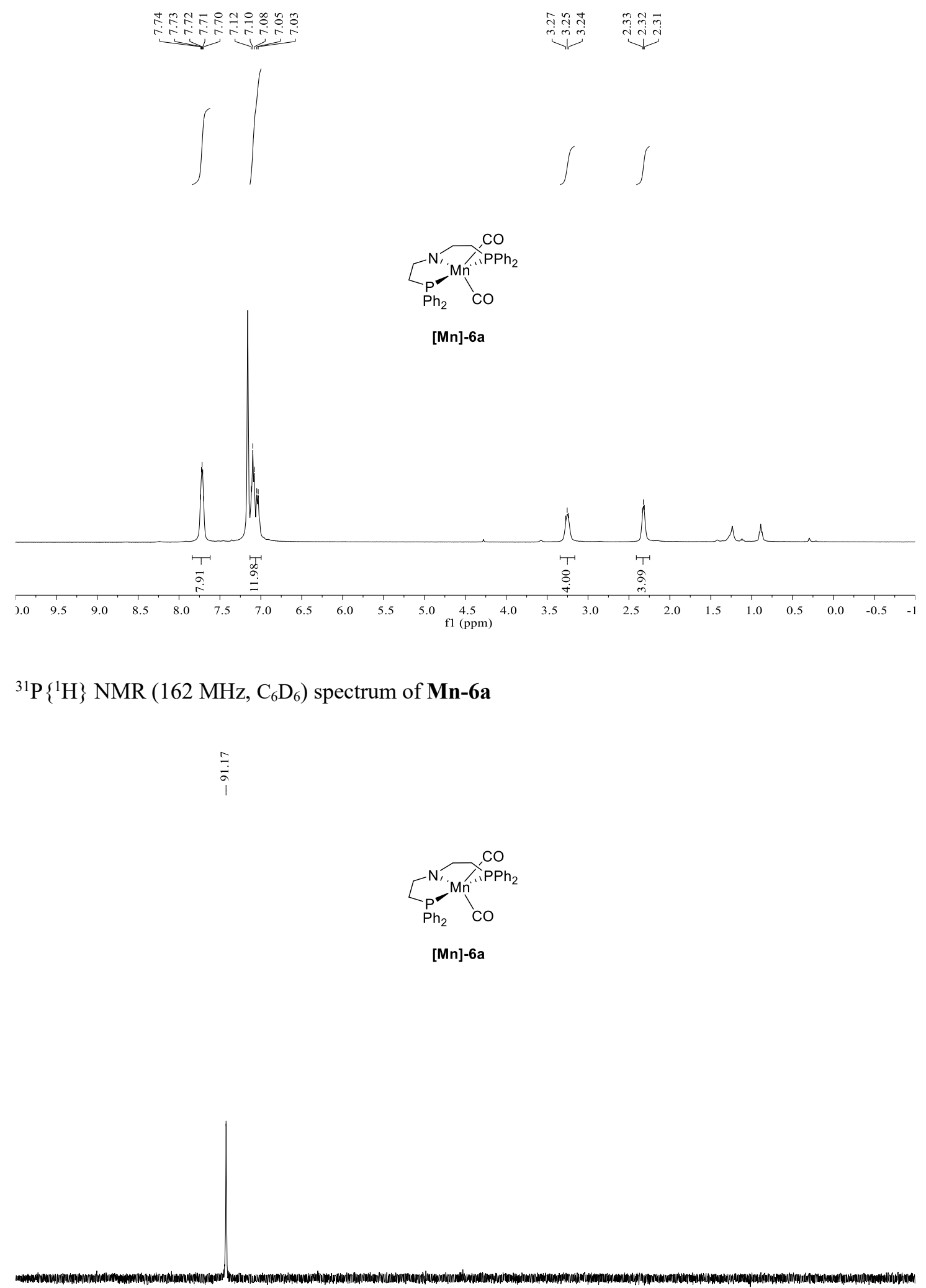

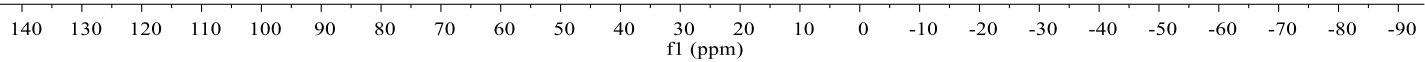


${ }^{1} \mathrm{H}$ NMR $\left(400 \mathrm{MHz}, \mathrm{CDCl}_{3}\right)$ spectrum of $7 \mathbf{a}$

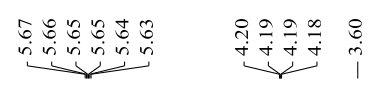

$\underbrace{1}$

$\underbrace{10}$
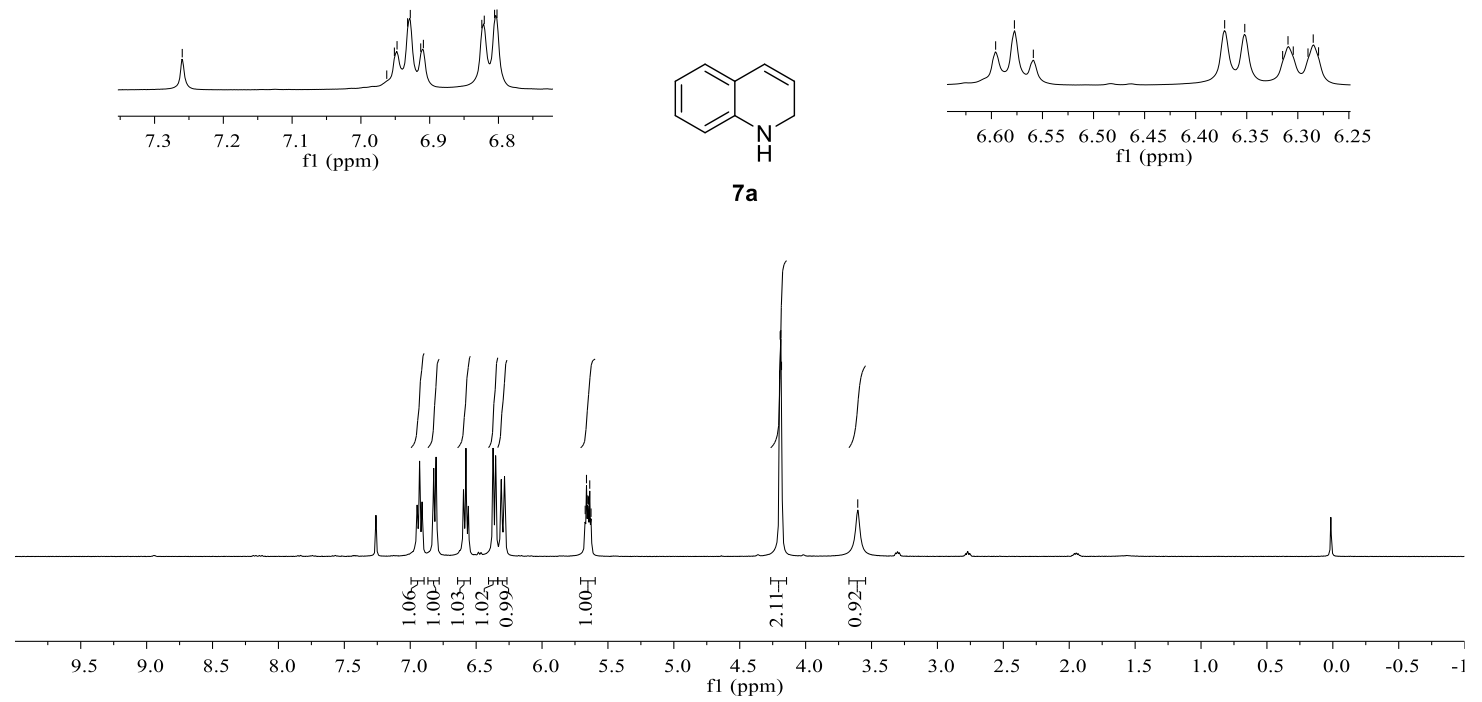

${ }^{13} \mathrm{C}\left\{{ }^{1} \mathrm{H}\right\}$ NMR $\left(101 \mathrm{MHz}, \mathrm{CDCl}_{3}\right)$ spectrum of 7a

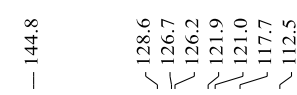

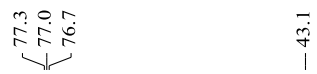

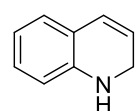

$7 a$

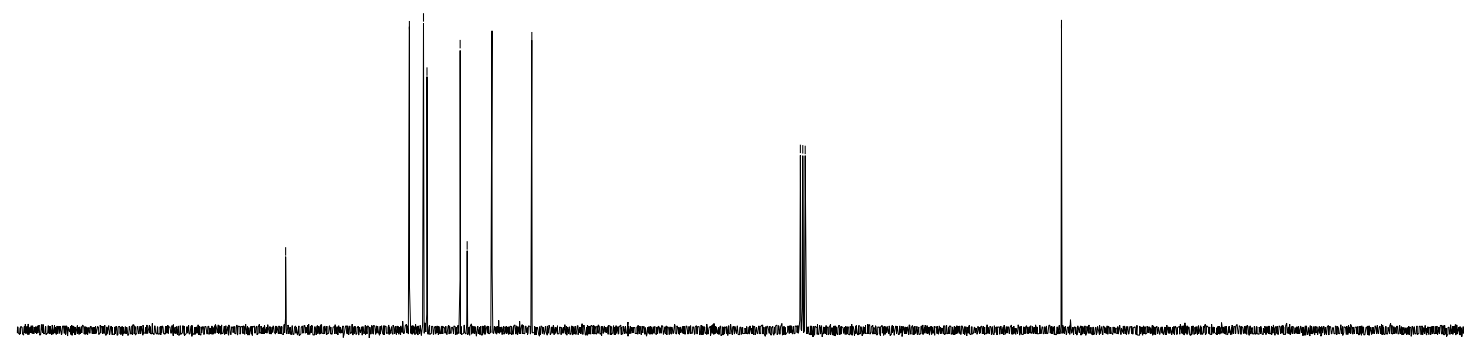

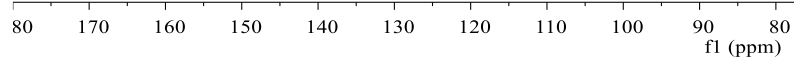


${ }^{1} \mathrm{H}$ NMR (400 MHz, $\mathrm{CDCl}_{3}$ ) spectrum of $\mathbf{1 6}$

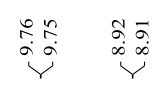
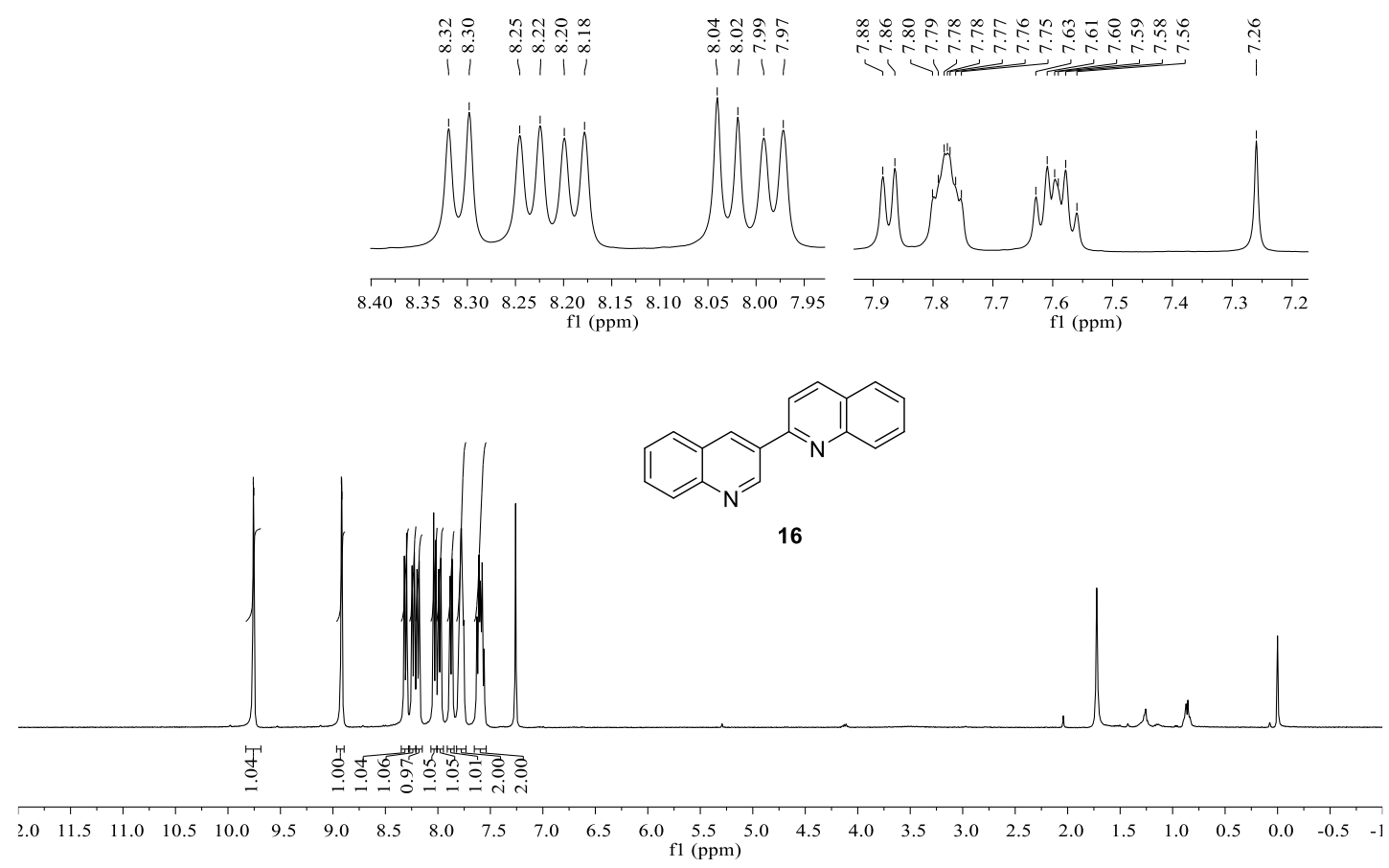

${ }^{13} \mathrm{C}\left\{{ }^{1} \mathrm{H}\right\}$ NMR $\left(101 \mathrm{MHz}, \mathrm{CDCl}_{3}\right)$ spectrum of $\mathbf{1 6}$

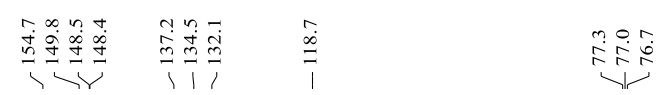

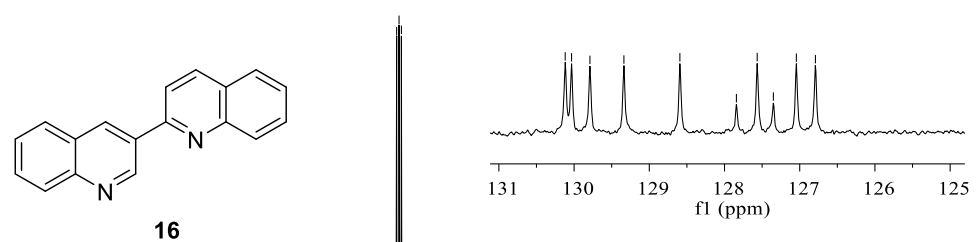

16

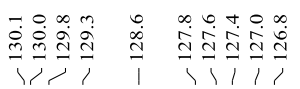

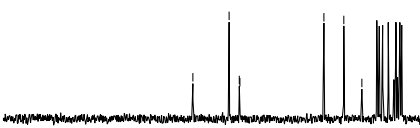

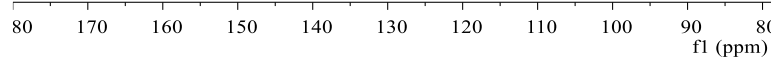


${ }^{1} \mathrm{H}$ NMR (400 MHz, $\mathrm{CDCl}_{3}$ ) spectrum of $\mathbf{1 7}$

$\underset{\substack{\overrightarrow{0} \\ \infty}}{\substack{\infty \\ \infty}}$

$\stackrel{i}{i}$
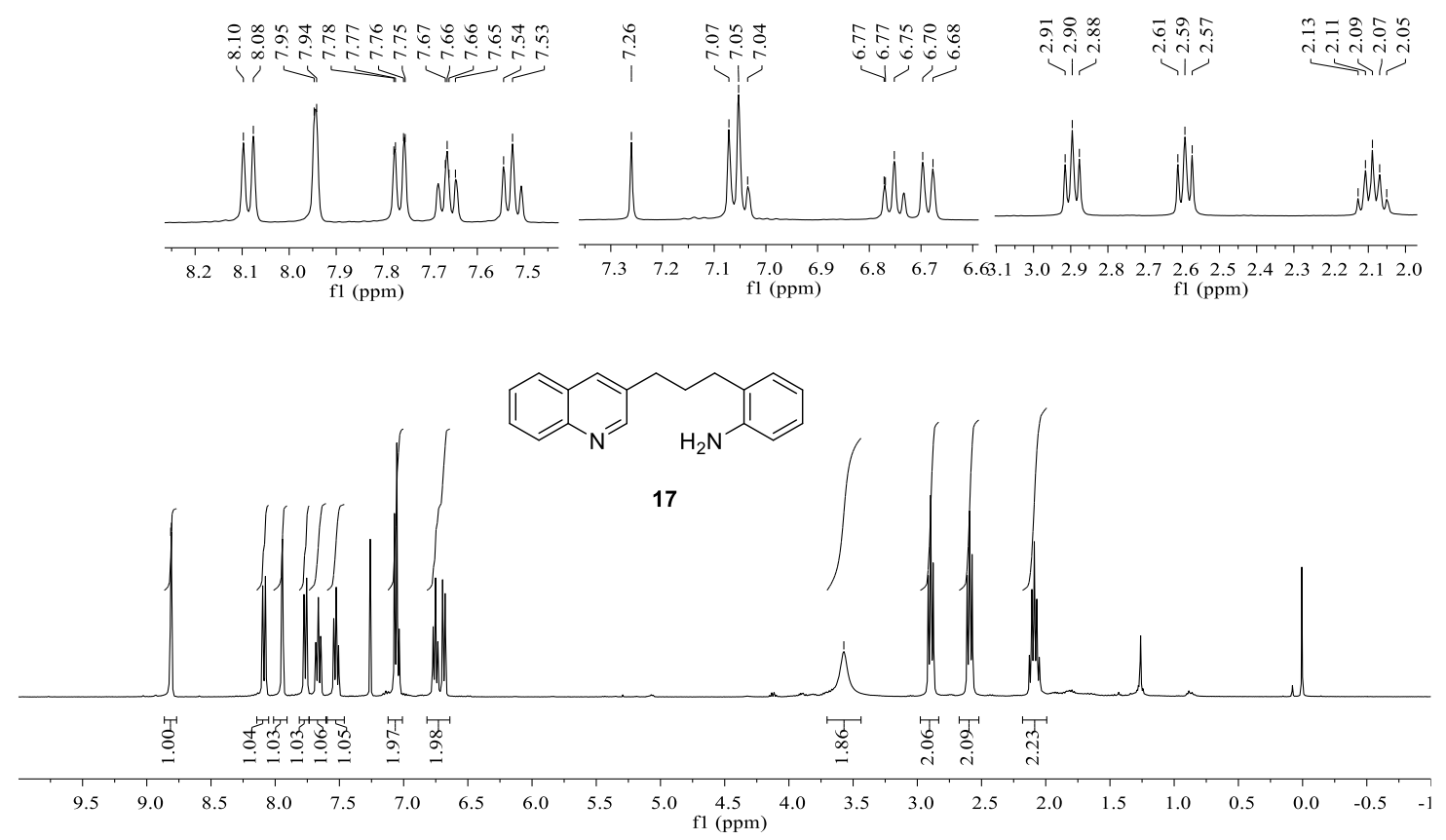

${ }^{13} \mathrm{C}\left\{{ }^{1} \mathrm{H}\right\}$ NMR $\left(101 \mathrm{MHz}, \mathrm{CDCl}_{3}\right)$ spectrum of $\mathbf{1 7}$

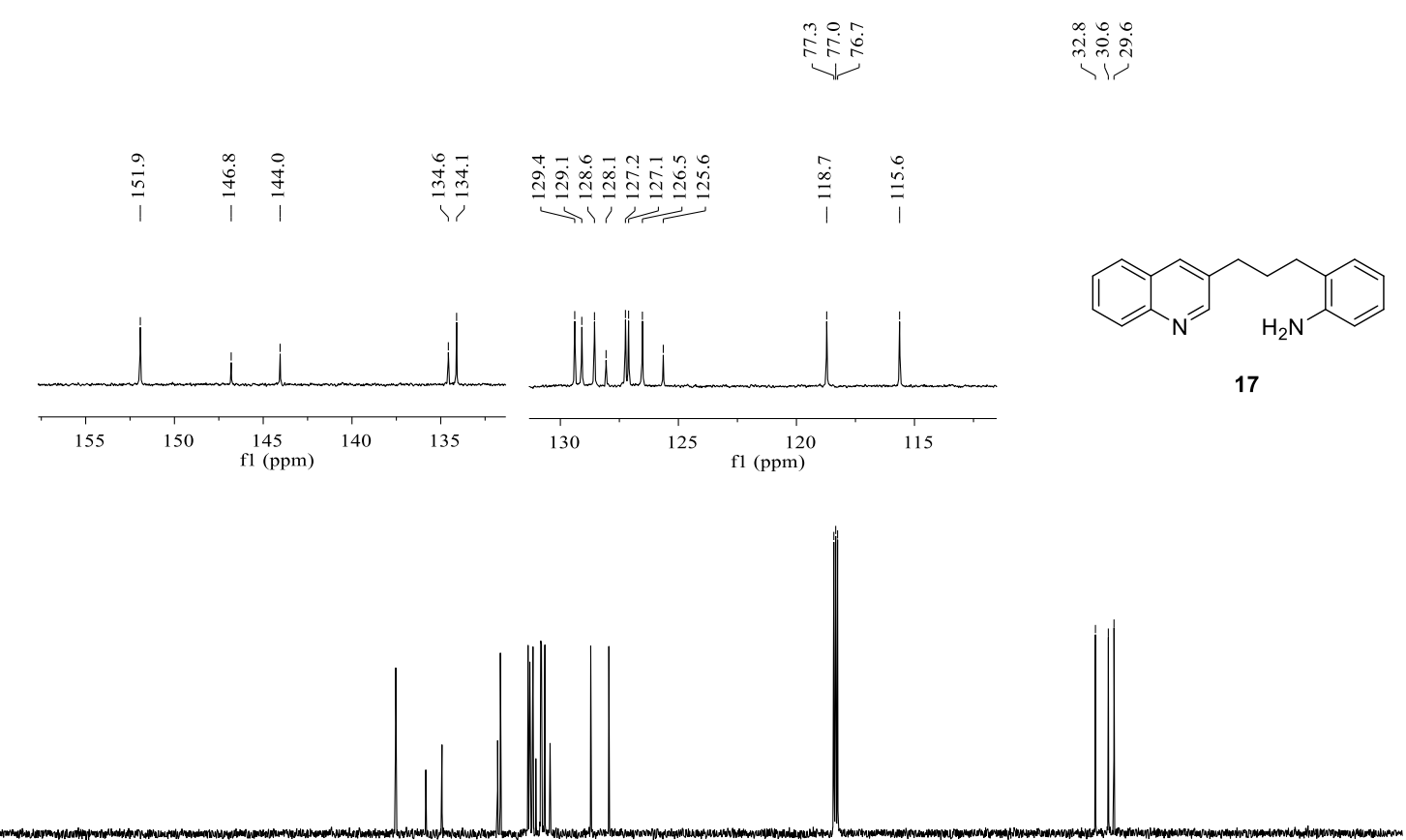

$\begin{array}{llllllllllllllllllllllll}1 & 210 & 200 & 190 & 180 & 170 & 160 & 150 & 140 & 130 & 120 & 110 & 100 & 10 & 80 & 70 & 60 & 50 & 40 & 30 & 20 & 10 & 0 & -10\end{array}$ 
${ }^{1} \mathrm{H}$ NMR $\left(400 \mathrm{MHz}, \mathrm{CDCl}_{3}\right)$ spectrum of $\mathbf{2 b}$

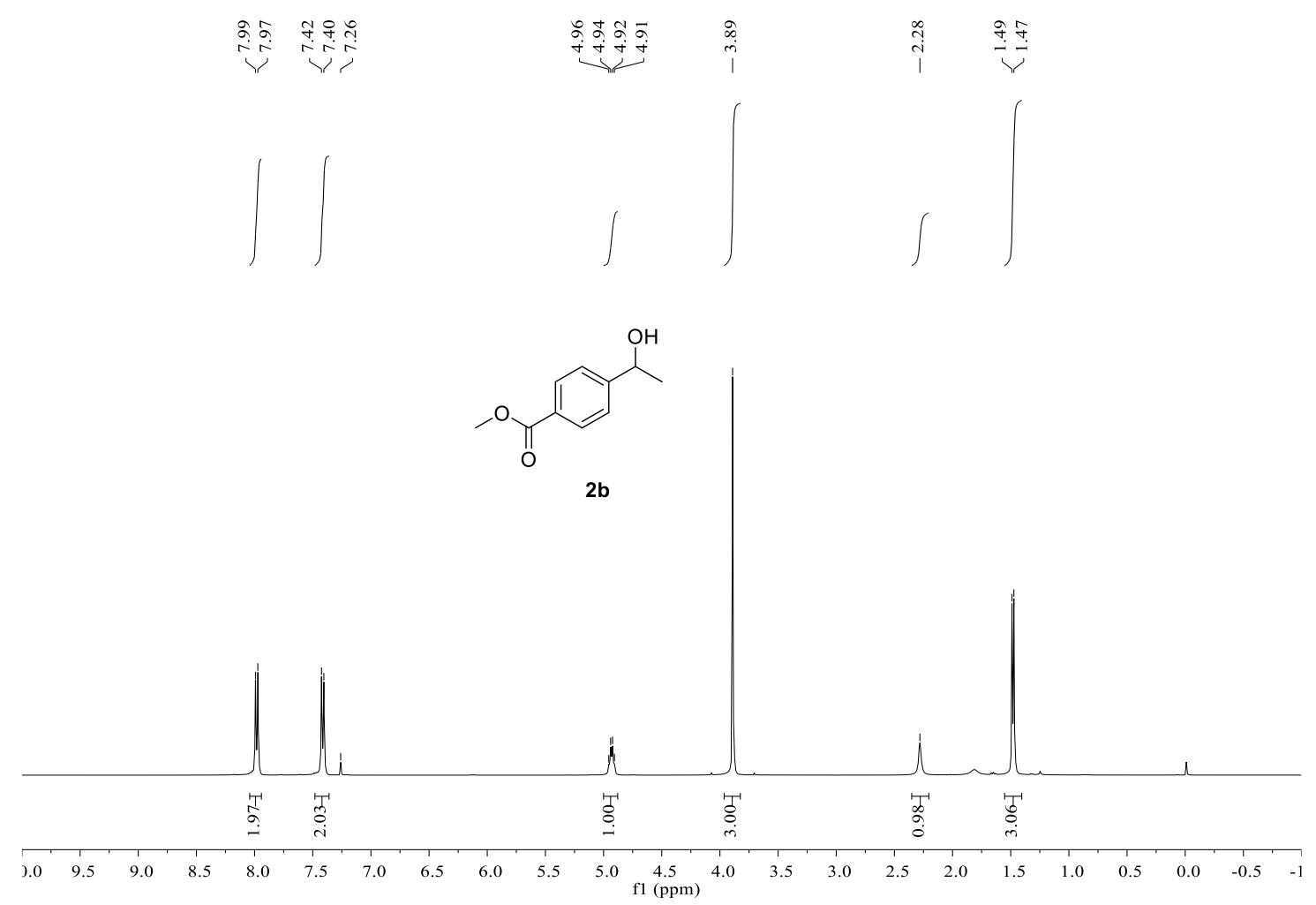

${ }^{13} \mathrm{C}\left\{{ }^{1} \mathrm{H}\right\}$ NMR $\left(101 \mathrm{MHz}, \mathrm{CDCl}_{3}\right)$ spectrum of $\mathbf{2 b}$

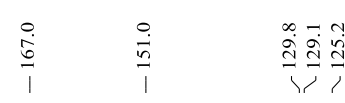

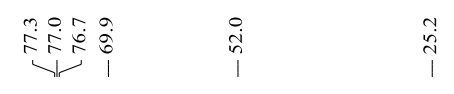

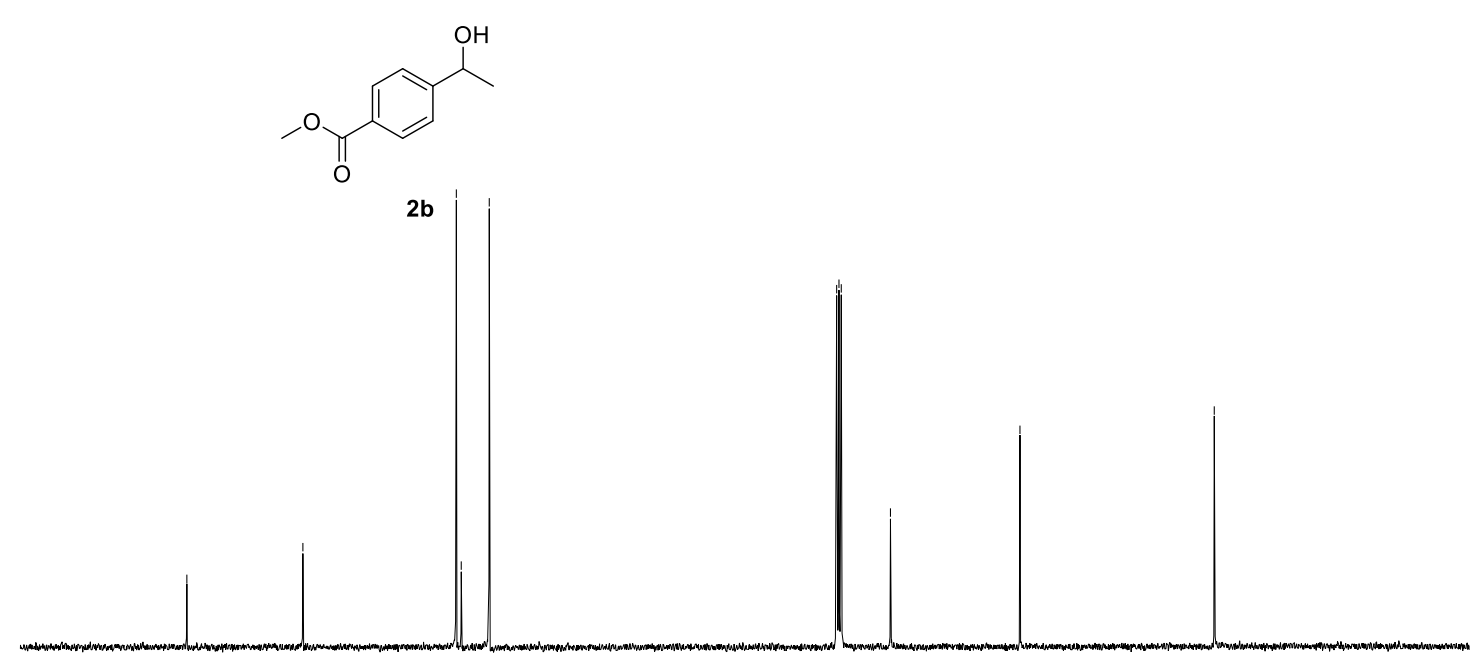

\begin{tabular}{rlllllllllllllllllllll}
\hline 90 & 180 & 170 & 160 & 150 & 140 & 130 & 120 & 110 & 100 & $\begin{array}{c}90 \\
\mathrm{f}(\mathrm{ppm})\end{array}$ & 80 & 70 & 60 & 50 & 40 & 30 & 20 & 10 & 0 & -
\end{tabular} 
${ }^{1} \mathrm{H}$ NMR $\left(400 \mathrm{MHz}, \mathrm{CDCl}_{3}\right)$ spectrum of $\mathbf{2 c}$

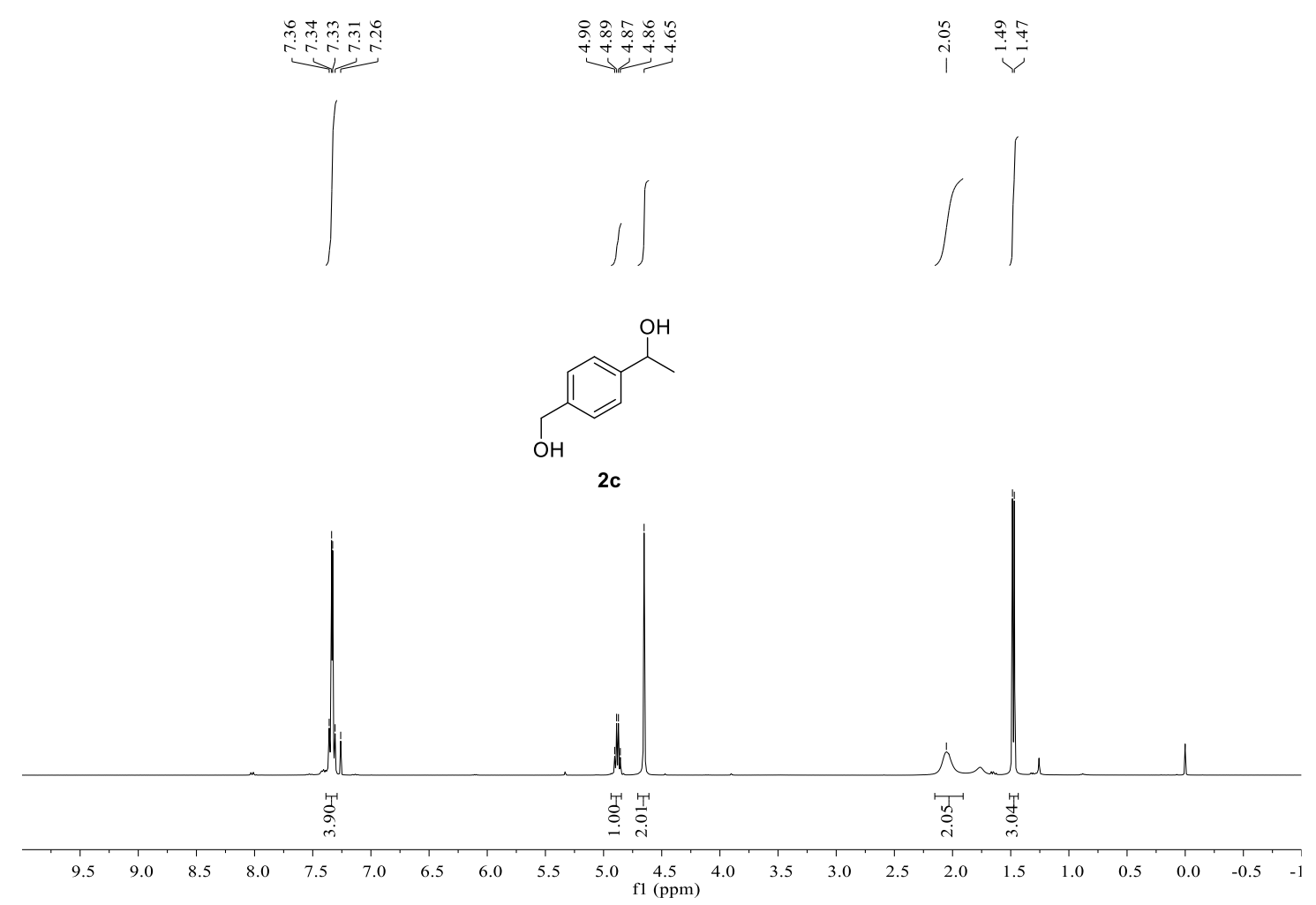

${ }^{13} \mathrm{C}\left\{{ }^{1} \mathrm{H}\right\}$ NMR $\left(101 \mathrm{MHz}, \mathrm{CDCl}_{3}\right)$ spectrum of $\mathbf{2 c}$

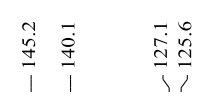

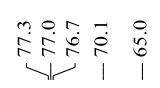

$\vec{i}$
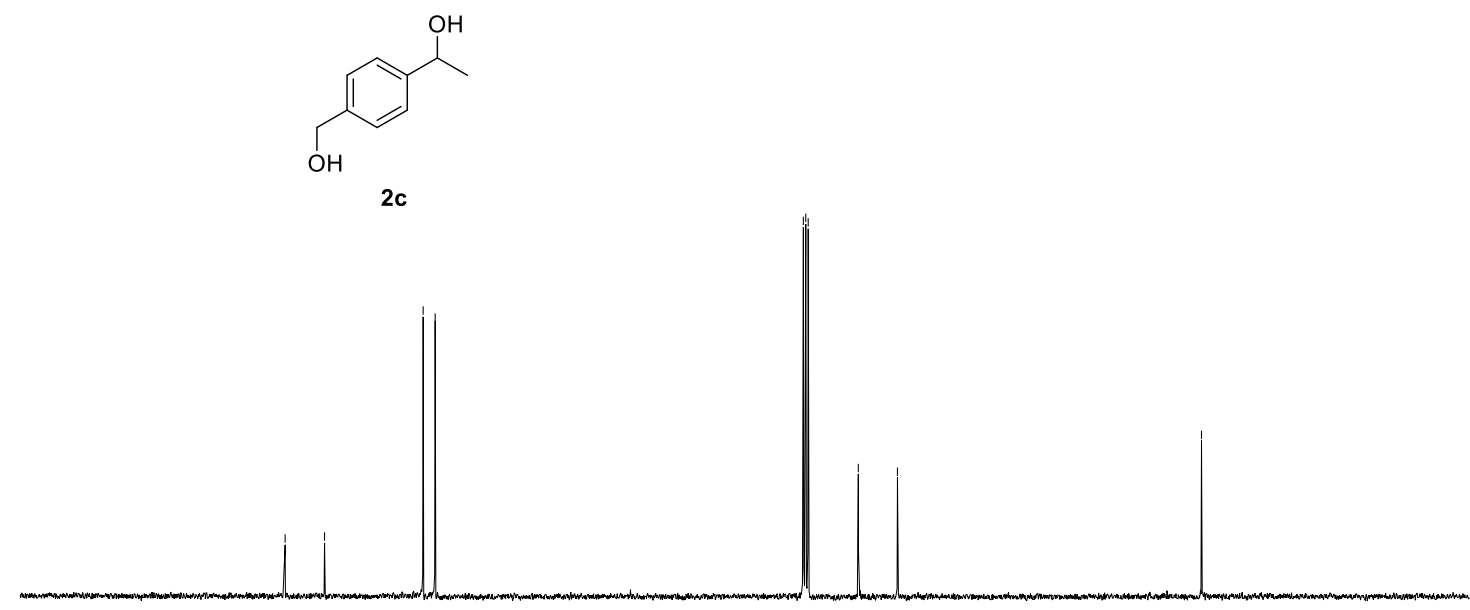

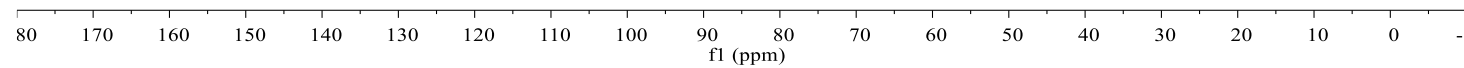

